\title{
Electron transfer kinetic studies of 1-electron, 1-proton redox couples attached to electrodes
}

Robert M. Haddox

West Virginia University

Follow this and additional works at: https://researchrepository.wvu.edu/etd

\section{Recommended Citation}

Haddox, Robert M., "Electron transfer kinetic studies of 1-electron, 1-proton redox couples attached to electrodes" (2002). Graduate Theses, Dissertations, and Problem Reports. 1703.

https://researchrepository.wvu.edu/etd/1703

This Dissertation is protected by copyright and/or related rights. It has been brought to you by the The Research Repository @ WVU with permission from the rights-holder(s). You are free to use this Dissertation in any way that is permitted by the copyright and related rights legislation that applies to your use. For other uses you must obtain permission from the rights-holder(s) directly, unless additional rights are indicated by a Creative Commons license in the record and/ or on the work itself. This Dissertation has been accepted for inclusion in WVU Graduate Theses, Dissertations, and Problem Reports collection by an authorized administrator of The Research Repository @ WVU.

For more information, please contact researchrepository@mail.wvu.edu. 


\title{
Electron Transfer Kinetic Studies of 1-Electron, 1-Proton Redox Couples Attached to Electrodes
}

\author{
Robert M. Haddox
}

Dissertation Submitted to the College of Arts and Sciences at West Virginia University in partial fulfillment of the requirements for the degree of Doctor of Philosophy In Chemistry

\author{
Harry O. Finklea, Ph. D., Chair, \\ Fred L. King, Ph. D., \\ Ronald B. Smart, Ph. D., \\ Alan M. Stolzenberg, Ph. D., \\ John W. Zondlo, Ph. D. \\ Department of Chemistry \\ Morgantown, West Virginia \\ 2002
}

Keywords: Cyclic Voltammetry, Proton Coupled Electron Transfer, 1e1H, Kinetics of Heterogeneous Electron Transfer, Alkanethiols, Self-Assembled Monolayers, Marcus DOS Theory, Transfer Coefficient, Galvinol, Uncompensated Resistance, 


\section{Abstract \\ Electron Transfer Kinetic Studies of 1-Electron, 1-Proton Redox Couples Attached to Electrodes}

Robert M. Haddox

Proton Coupled Electron Transfer (PCET) reactions play a vital role in a variety of biological and chemical processes. Prior work by E. Laviron developed the theory of proton coupled electron transfer kinetics based on a stepwise mechanism with the transfer coefficient for electron transfer remaining constant at all overpotentials. The present work explores the consequences of a potential-dependent transfer coefficient as applied to redox couples attached to electrodes. Specifically, this work focuses on the 1-electron, 1-proton $(1 \mathrm{e} 1 \mathrm{H})$ system. Derivation of the potential-dependence of the transfer coefficient comes from the Marcus density-of-states model of heterogeneous electron transfer. Two 1e1H systems are examined. Galvinol is an organic redox couple that forms a stable free radical, galvinoxyl, and exhibits $1 \mathrm{e} 1 \mathrm{H}$ behavior. The thermodynamics and kinetics of electron transfer for a galvinol-terminated alkanethiol, adsorbed to a gold electrode, are explored. Also discussed is the inorganic $1 \mathrm{e} 1 \mathrm{H}$ redox couple, $\left[\mathrm{Os}(\mathrm{bpy})_{2}(4-\mathrm{AMP}) \mathrm{OH}_{2}\right]^{2+}$, attached to an alkanethiol that is adsorbed to a gold electrode. Results show that both the galvinol and the osmium redox couples behave as predicted by the thermodynamic model. However, for both systems, experimental results deviate significantly from the kinetic model. 


\section{Acknowledgements}

First and foremost I would like to express my deepest thanks to my family, specifically to my parents, whose love and support (emotional as well as financial) have been constant and unconditional throughout this rollercoaster called education. This achievement would not have been possible without you, and I love you both.

I'd like to thank Professor Harry O. Finklea, mentor and occasional novel borrower, for allowing me the freedom to work on my own terms while keeping me pointed in the right direction. I would also like to thank the past and current members of the Finklea group (Kwansik, Dmitri, Theresa, Yi, Chris, and Mark) for making the windowless walls of $550 \mathrm{CRL}$ a little bit brighter.

Finally, a large part of a successful education has absolutely nothing to do with school at all, but rather what one does away from school. For this, I would like to thank my close friends (you know who you are) who have been around for all the football games, road trips, and Buffett concerts. May there be many more.

Go Mountaineers. Notre Dame Sucks.

This work was supported in part by the National Science Foundation grant CHE9711779. I would like to acknowledge Prof. David Shultz and Prof. Debbie Mohler for their contribution to this project. 
Table of Contents

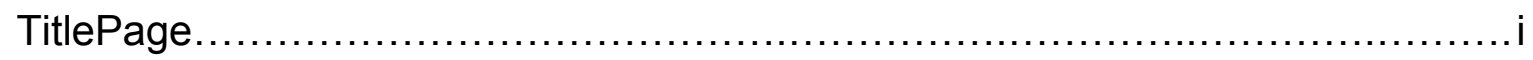

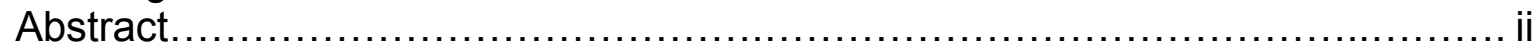

Acknowledgements..................

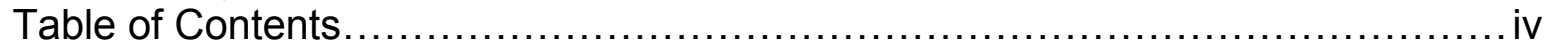

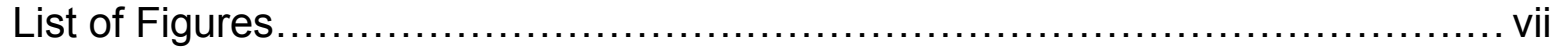

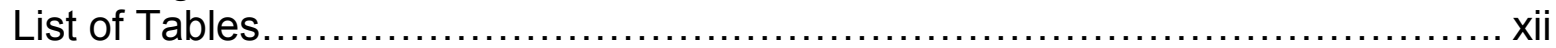

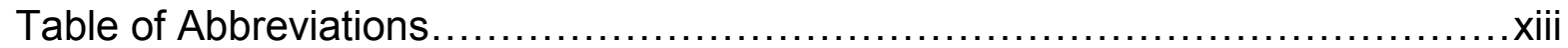

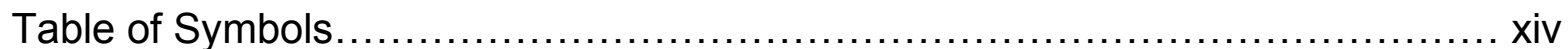

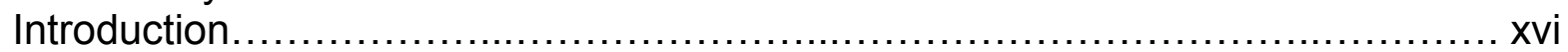

Chapter 1 Introduction to Electron Transfer Theory ............................ 2

1.1 Electron transfer at an electrode interface in solution: The Butler-Volmer

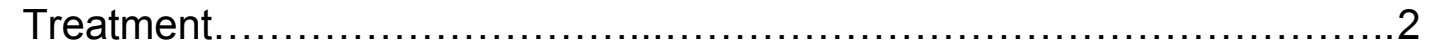

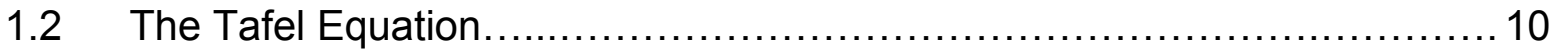

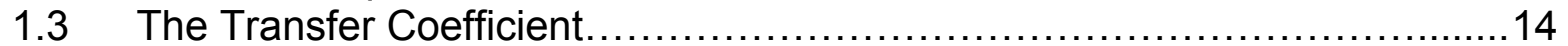

1.4 Deficiencies of the Butler-Volmer Approach................................ 15

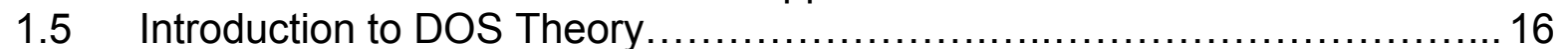

1.6 Elementary Steps in Marcus DOS Theory................................... 19

1.7 Derivation of Rate Expressions of DOS Theory - The Marcus Approach....23

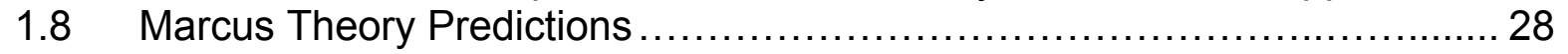

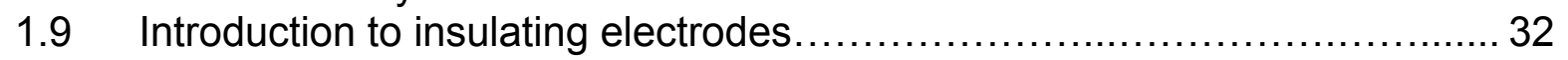

1.10 Redox centers attached to self-assembled monolayers......................34

1.11 Introduction to Proton Coupled Electron Transfer Reactions.................. 37

1.12 Kinetic Predictions using Laviron Theory ..................................... 43

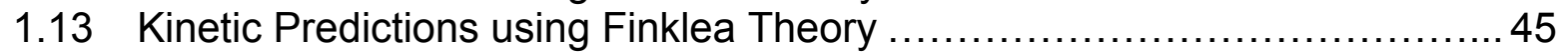

1.14 Direction and Goals of this Research Project.............................. 48

Chapter 2 Instrumentation and General Experimental.................... 53

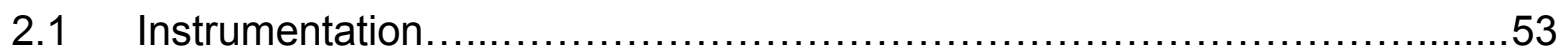

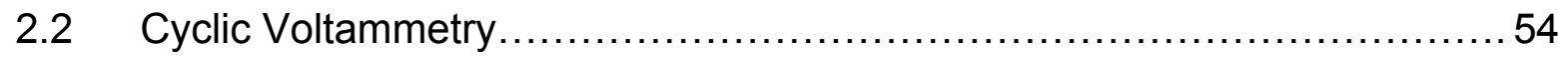

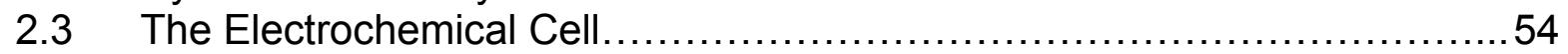

2.4 The Electrodes - Synthesis, Cleaning, and Characterization................ 58

2.5 Monolayer Deposition, General Discussion................................. 61

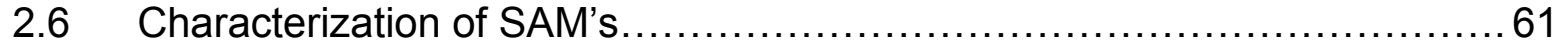

2.7 Thermodynamic Characterization of Attached Redox Centers

2.8 Kinetic Characterization of Redox Centers on SAM's ........................6 67

$2.9 \quad$ iR Drop and Uncompensated Resistance...................................... 69

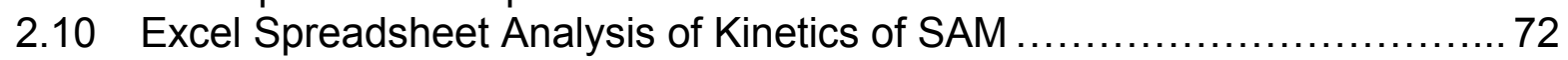




\section{Chapter 3 Proton Coupled Electron Transfer Kinetic Studies of}

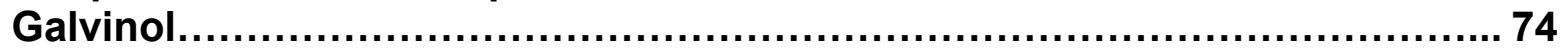

3.1 Introduction to Galvinol.................................................... 74

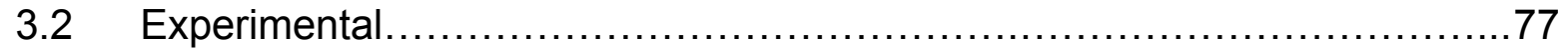

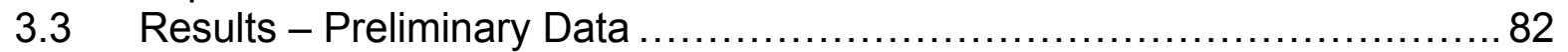

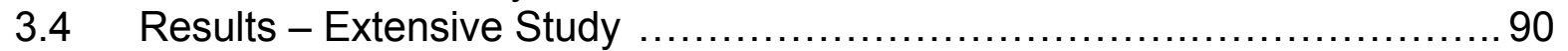

3.5 Discussion of Results.................................................... 103

Chapter 4 Proton Coupled Electron Transfer Kinetic Studies of

Polypyridyl Osmium Complexes......................................................... 109

$4.1 \quad$ Introduction - The Osmium System.......................................... 109

4.2 Experimental - The Osmium System......................................... 114

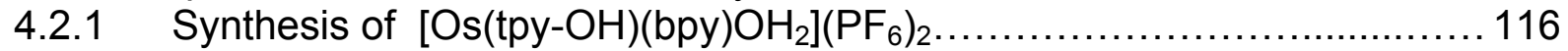

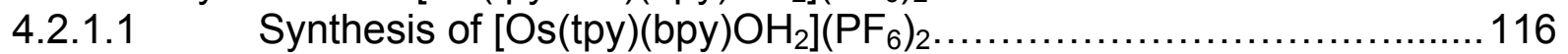

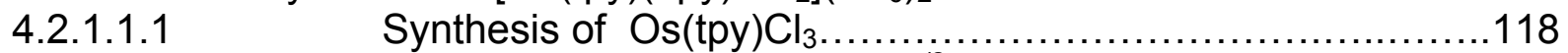

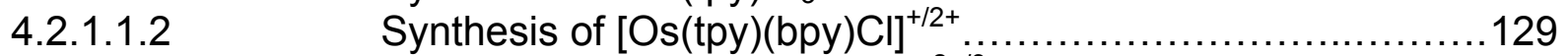

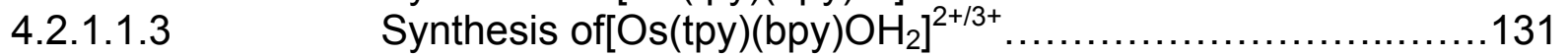

4.2.1.2 Synthesis of the modified terpyridine ligand..........................137

4.2.1.3 Synthesis of $\left[\mathrm{Os}(\mathrm{tpy}-\mathrm{OH})(\mathrm{bpy}) \mathrm{Cl}^{2+/ 3+}\right.$ and

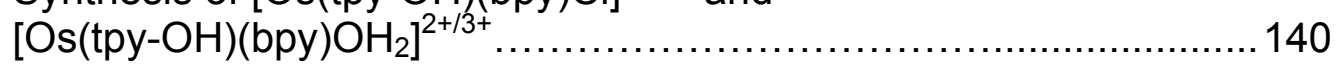

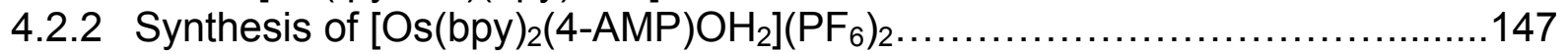

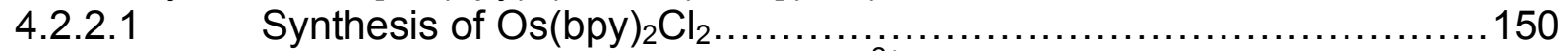

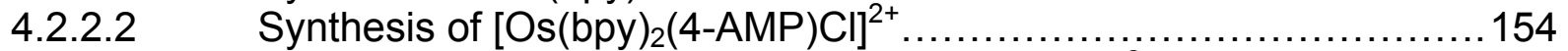

4.2.2.3 Synthesis of $\mathrm{Os}(\mathrm{bpy})_{2} \mathrm{CO}_{3}$ and $\left[\mathrm{Os}(\mathrm{bpy})_{2}\left(\mathrm{OH}_{2}\right)_{2}\right]^{2+} \ldots \ldots \ldots \ldots \ldots \ldots \ldots \ldots$

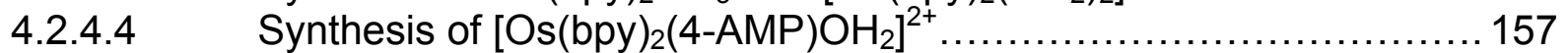

4.2.3 Preparation and Deposition of Monolayers................................. 163

4.2.4 Coupling of Redox Centers to Monolayers.................................164

4.3 Results - The Osmium System........................................... 166

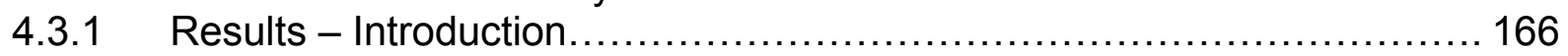

4.3.2 Results - The $\left[\mathrm{Os}(\text { tpy-OH)(bpy)Cl] }]^{+}\right.$System............................... 167

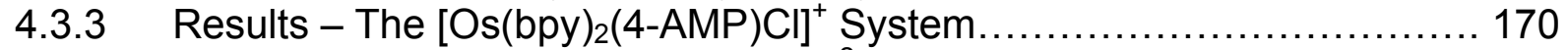

4.3.4 Results - The $\left[\mathrm{Os}\left(\text { tpy-OH)(bpy) } \mathrm{OH}_{2}\right]^{2+}\right.$ System.......................... 171

4.3.5 Results - The $\left[\mathrm{Os}(\mathrm{bpy})_{2}(4-\mathrm{AMP}) \mathrm{OH}_{2}\right]^{2+}$ System........................ 178

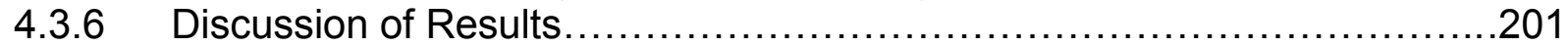


Chapter 5 Final Discussion and Conclusions................................... 207

5.1 Discussion of the deviation of ks,app for Galvinol and the Osmium

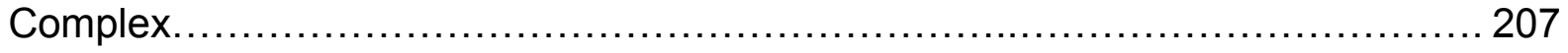

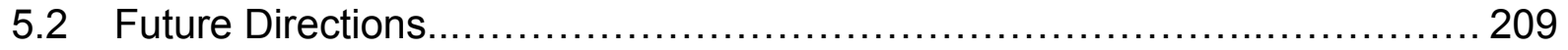

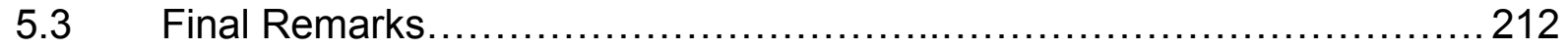

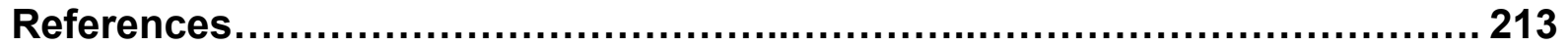




\section{List of Figures}

Figure 1.1. 1 Free energy diagram of an Electron Transfer Process .................... 4

Figure 1.1. 2 The effect of electrode potential on a Reaction Coordinate diagram . . 6

Figure 1.3. 1 The effect of the transfer coefficient on a Reaction Coordinate

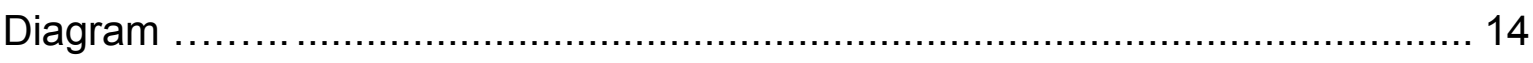

Figure 1.7. 1 Marcus Density of States Diagrams at various overpotential ........... 24

Figure 1.7. 2 Dependence of reductive current on negative overpotential............ 26

Figure 1.8. 1 Dependence of Curvature of Tafel Plot on Reorganization Energy ... 28

Figure 1.8. 2 Dependence of Transfer Coefficeint on Reorganization Energy ........ 30

Figure 1.10. 1 Diagram of monolayer with attached redox centers adsorbed to an electrode. 34

Figure 1.11. 1 1e1H Square Scheme ........................................... 38

Figure 1.12. $1 \quad \log \mathrm{k}_{\mathrm{s}} \mathrm{vs} \mathrm{pH}$ using Laviron analysis ................................... 44

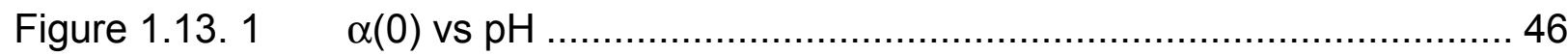

Figure 1.13. $2 \quad \mathrm{CV}$ and Tafel Plot of system where $\mathrm{pK}_{\mathrm{a} 1}<\mathrm{pH}<\mathrm{pK}_{\text {mid. }} \ldots \ldots \ldots \ldots \ldots . \ldots . \ldots$

Figure 1.13. $3 \quad \mathrm{CV}$ and Tafel Plot of system where $\mathrm{pK}_{\mathrm{a} 2}>\mathrm{pH}>\mathrm{pK}_{\text {mid. }} \ldots \ldots \ldots \ldots \ldots \ldots . \ldots$

Figure 1.13. $4 \quad \log \mathrm{k}_{\mathrm{s}, \mathrm{app}} \mathrm{vs} \mathrm{pH}$ using the Finklea model ............................50

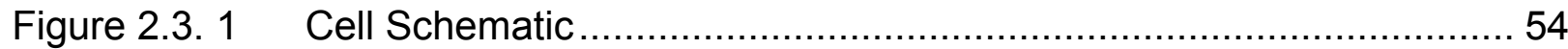

Figure 2.3. 2 The Electrochemical Flow Cell................................................ 56

Figure 2.7. 1 Faradaic and Nonfaradaic components of a CV ...........................63 63

Figure 2.9. 1 RC Circuit showing uncompensated resistance component ............68

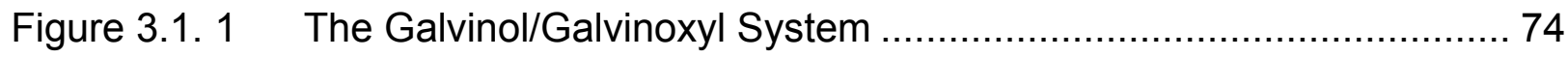


Figure 3.4. $1 \quad$ Typical Reversible Voltammogram ............................................. 93

Figure 3.4. 2 Apparent Formal Potential vs $\mathrm{pH}$................................................. 94

Figure 3.4. 3 Average $\mathrm{k}_{\mathrm{s}, \mathrm{app}} \mathrm{vs} \mathrm{pH}$ - extracted at formal potential....................... 95

Figure 3.4. 4 Average $\mathrm{k}_{\mathrm{s} \text {,app }} \mathrm{vs} \mathrm{pH}$ - extracted from Tafel Analysis....................... 96

Figure 3.4. $5 \quad$ Example Tafel Plot................................................................... 99

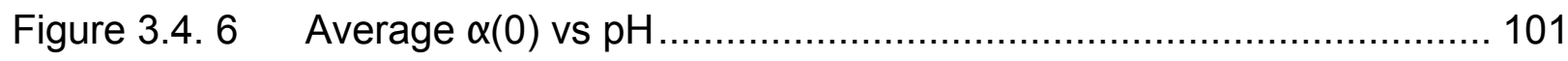

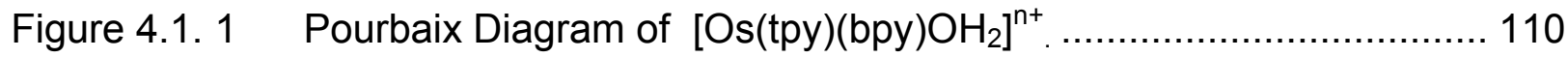

Figure 4.1. 2 Various Polypyridyl ligands ………............................................ 113

Figure 4.1. 3 Dr. Mohler's Modified Terpyridine Ligand ...................................... 114

Figure 4.2.1.1. 1 Flowchart of $\left[\mathrm{Os}(\mathrm{tpy})(\mathrm{bpy}) \mathrm{OH}_{2}\right]^{2+/ 3+}$ Synthesis........................ 116

Figure 4.2.1.1.1. 1 Reference UV-VIS Spectrum of Os(tpy)Cl $l_{3}$ in DMF................ 118

Figure 4.2.1.1.1. 2 Reference Voltammogram of Os(tpy) $\mathrm{Cl}_{3}$ in $0.1 \mathrm{M}$ tertbutylammonium hydroxide/DMF

Figure 4.2.1.1.1. 3 UV-VIS Spectra of $\mathrm{Os}(\mathrm{tpy}) \mathrm{Cl}_{3}$ in DMF - Synthesis A3 with high purity grade DMF.....

Figure 4.2.1.1.1. 4 Voltammogram of $\mathrm{Os}(\mathrm{tpy}) \mathrm{Cl}_{3}$ in $0.1 \mathrm{M}$ tetramethylammonium tetrafluoroborate/DMF- Synthesis A3 with high purity grade DMF.

Figure 4.2.1.1.1. 5 Voltammogram of $\mathrm{Os}(\mathrm{tpy}) \mathrm{Cl}_{3}$ in $0.1 \mathrm{M}$ tetramethylammonium tetrafluoroborate/DMF- Synthesis A4 with high purity grade DMF

Figure 4.2.1.1.2. 1 UV-VIS Spectra of $\left[\mathrm{Os}(\mathrm{tpy})(\mathrm{bpy}) \mathrm{Cl}_{\mathrm{P}} \mathrm{PF}_{6}\right.$ in $\mathrm{CH}_{3} \mathrm{CN}-$ Synthesis A5

Figure 4.2.1.1.2. 2 Voltammogram of $\left[\mathrm{Os}\left(\right.\right.$ tpy)(bpy)Cl]PF 6 in $0.1 \mathrm{NaClO} / \mathrm{CH}_{3} \mathrm{CN}-$ Synthesis A5

Figure 4.2.1.1.3. 1 UV-VIS Spectra of $\left[\mathrm{Os}(\mathrm{tpy})(\mathrm{bpy}) \mathrm{OH}_{2}\right]\left(\mathrm{PF}_{6}\right)_{2}$ in $\mathrm{H}_{2} \mathrm{O}$ Synthesis $\mathrm{A} 6$ using Distilled TFMS acid 
Figure 4.2.1.1.3. 2 Voltammogram of $\left[\mathrm{Os}(\mathrm{tpy})(\mathrm{bpy}) \mathrm{OH}_{2}\right]\left(\mathrm{PF}_{6}\right)_{2}$ in $0.1 \mathrm{M} \mathrm{H}_{2} \mathrm{SO}_{4}+0.1$ $\mathrm{M} \mathrm{H}_{3} \mathrm{PO}_{4}$ aqueous solution at $\mathrm{pH} 3$ - Synthesis A6 using Distilled TFMS acid ... 135

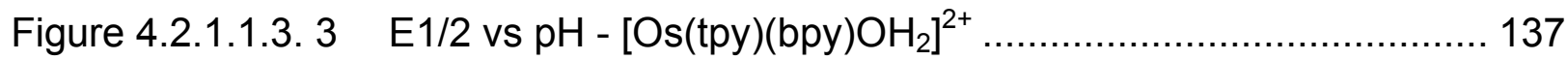

Figure 4.2.1.2. $1 \quad{ }^{1} \mathrm{H}-\mathrm{NMR}$ of tpy- $\mathrm{OH}$ in deuterated methanol .............................. 138

Figure 4.2.1.3. 1 Voltammogram of $\mathrm{Os}(\mathrm{tpy}-\mathrm{OH}) \mathrm{Cl}_{3}$ in $0.1 \mathrm{M}$ tetramethylammonium tetrafluoroborate/DMF ........................................................................... 140

Figure 4.2.1.3. 2 Voltammogram of $\left[\mathrm{Os}(\mathrm{tpy}-\mathrm{OH})(\mathrm{bpy}) \mathrm{Cl}_{\mathrm{PF}}\right.$ in $0.1 \mathrm{M}$ $\mathrm{NaClO} / 4 / \mathrm{CH}_{3} \mathrm{CN}$.

Figure 4.2.1.3. 3 Mass Spectrum of $\left[\mathrm{Os}\left(\right.\right.$ tpy-OH)(bpy)Cl]PF 6 in $\mathrm{CH}_{3} \mathrm{CN} \ldots \ldots \ldots \ldots \ldots . . .142$

Figure 4.2.1.3. $4 \quad$ Isotopic Distribution of [Os(tpy-OH)(bpy)Cl]PF $6 \ldots \ldots \ldots \ldots \ldots \ldots \ldots . . .144$

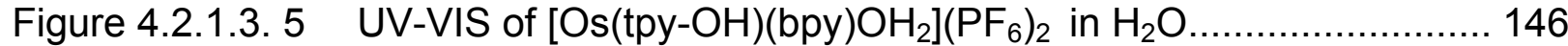

Figure 4.2.1.3. 6 Voltammogram of $\left[\mathrm{Os}\right.$ (tpy-OH)(bpy)OH $\left.\mathrm{OH}_{2}\right]\left(\mathrm{PF}_{6}\right)_{2}$ in $0.1 \mathrm{M} \mathrm{H}_{2} \mathrm{SO}_{4}$ $+0.1 \mathrm{M} \mathrm{H}_{3} \mathrm{PO}_{4}$ aqueous solution at $\mathrm{pH} 3$

Figure 4.2.1.3. 7 Mass Spectrum of $\left[\mathrm{Os}(\mathrm{tpy}-\mathrm{OH})(\mathrm{bpy}) \mathrm{OH}_{2}\right]\left(\mathrm{PF}_{6}\right)_{2}$ in $\mathrm{H}_{2} \mathrm{O} \ldots \ldots \ldots \ldots . . .148$

Figure 4.2.2. $1 \quad$ Flowchart of $\left[\mathrm{Os}(\mathrm{bpy})_{2}(4-\mathrm{AMP}) \mathrm{OH}_{2}\right]\left(\mathrm{PF}_{6}\right)_{2}$ Synthesis ................. 150

Figure 4.2.2.1. $1 \quad$ UV-VIS Spectrum of $\mathrm{Os}(\mathrm{bpy})_{2} \mathrm{Cl}_{2}$ in $\mathrm{CH}_{3} \mathrm{CN} \ldots \ldots \ldots \ldots \ldots \ldots \ldots \ldots \ldots . . . . . . . . . . . . .151$

Figure 4.2.2.1. 2 Voltammogram of $\mathrm{Os}(\mathrm{bpy})_{2} \mathrm{Cl}_{2}$ in $0.1 \mathrm{M}$ tetramethylammonium tetrafluoroborate $/ \mathrm{CH}_{3} \mathrm{CN}$.

Figure 4.2.2.2. 1 Voltammogram of $\left[\mathrm{Os}(\mathrm{bpy})_{2}(4-\mathrm{AMP}) \mathrm{Cl}\right]^{+}$in $0.1 \mathrm{M} \mathrm{NaClO}{ }_{4} / \mathrm{CH}_{3} \mathrm{CN}$ 154

Figure 4.2.2.3. 1 Voltammogram of $\left[\mathrm{Os}(\mathrm{bpy})_{2} \mathrm{OH}_{2}\right]_{2}{ }^{2+}$ from Literature

Figure 4.2.2.3. 2 Voltammogram of $\left[\mathrm{Os}(\mathrm{bpy})_{2} \mathrm{OH}_{2}\right]_{2}{ }^{2+}$ in $0.1 \mathrm{M} \mathrm{HClO}_{4}$ in aqueous solution, $\mathrm{pH} 1.08$

Figure 4.2.2.4. 1 Voltammograms of $\left[\mathrm{Os}(\mathrm{bpy})_{2}(4-\mathrm{AMP}) \mathrm{OH}\right]_{2}{ }^{2+}$ in $0.5 \mathrm{M} \mathrm{K}_{2} \mathrm{SO}_{4}+0.1$ $\mathrm{M}$ Britton-Robinson buffer in aqueous solution - Method B6. 
Figure 4.3.2. 1 Voltammogram of $[\mathrm{Os}(\mathrm{tpy}-\mathrm{OH})(\mathrm{bpy}) \mathrm{Cl}]^{+}$attached to $\mathrm{HS}\left(\mathrm{CH}_{2}\right)_{15} \mathrm{COOH}$ Monolayer on a Gold Electrode in $0.1 \mathrm{M} \mathrm{H}_{2} \mathrm{SO}_{4}+0.1 \mathrm{M} \mathrm{H}_{3} \mathrm{PO}_{4}$

aqueous solution. 167

Figure 4.3.2. 2 Voltammograms of $[\mathrm{Os}(\mathrm{tpy}-\mathrm{OH})(\mathrm{bpy}) \mathrm{Cl}]^{+}$at multiple Scan Rates in $0.1 \mathrm{M} \mathrm{H}_{2} \mathrm{SO}_{4}+0.1 \mathrm{M} \mathrm{H}_{3} \mathrm{PO}_{4}$ aqueous solution. 168

Figure 4.3.2. 3 Tafel Plots of $[\mathrm{Os}(\mathrm{tpy}-\mathrm{OH})(\mathrm{bpy}) \mathrm{Cl}]^{+}$ 170

Figure 4.3.3. 1 Voltammogram of $\left[\mathrm{Os}(\mathrm{bpy})_{2}(4-\mathrm{AMP}) \mathrm{Cl}^{+}\right.$attached to $\mathrm{HS}\left(\mathrm{CH}_{2}\right){ }_{15} \mathrm{COOH}$ Monolayer on a Gold Electrode in $1.0 \mathrm{M} \mathrm{NaClO}_{4}$

Figure 4.3.3. 2 Voltammograms of $[\mathrm{Os}(\mathrm{bpy}) 2(4-\mathrm{AMP}) \mathrm{Cl}]^{+}$in $1.0 \mathrm{M} \mathrm{NaClO}_{4}$, at multiple scan rates.

Figure 4.3.3. 3 Voltammograms of $[\mathrm{Os}(\mathrm{bpy}) 2(4-\mathrm{AMP}) \mathrm{Cl}]^{+}$at multiple $\mathrm{pH}$ 's at a scan rate of $.1 \mathrm{~V} / \mathrm{s}$.

Figure 4.3.3. $4 \quad$ Voltammograms of $[\mathrm{Os}(\mathrm{bpy}) 2(4-\mathrm{AMP}) \mathrm{Cl}]^{+}$at multiple $\mathrm{pH}$ 's at a scan rate of $1000 \mathrm{~V} / \mathrm{s}$.

Figure 4.3.4. $1 \mathrm{E} 1 / 2 \mathrm{vs} \mathrm{pH}$ for $\left[\mathrm{Os}(\mathrm{tpy}-\mathrm{OH})(\mathrm{bpy}) \mathrm{OH}_{2}\right]^{2+}$ 178

Figure 4.3.5. 1 Typical Voltammogram of $\left[\mathrm{Os}(\mathrm{bpy}) 2(4-\mathrm{AMP}) \mathrm{OH}_{2}\right]^{2+}$ attached to a $50 / 50 \mathrm{HS}\left(\mathrm{CH}_{2}\right)_{12} \mathrm{OH} / \mathrm{HS}\left(\mathrm{CH}_{2}\right)_{15} \mathrm{COOH}$ Monolayer, $0.5 \mathrm{M} \mathrm{K}_{2} \mathrm{SO}_{4}+0.1 \mathrm{M}$ BrittonRobinson buffer at $\mathrm{pH} 6.85$

Figure 4.3.5. $2 \quad \mathrm{E}_{\mathrm{r}}$ vs $\mathrm{pH}$ for $\left[\mathrm{Os}(\mathrm{bpy})_{2}(4-\mathrm{AMP}) \mathrm{OH}\right]_{2}{ }^{2+}$ attached to a $50 / 50$ $\mathrm{HS}\left(\mathrm{CH}_{2}\right)_{12} \mathrm{OH} / \mathrm{HS}\left(\mathrm{CH}_{2}\right){ }_{15} \mathrm{COOH}$ Monolayer

Figure 4.3.5. 3 Voltammograms at multiple scan rates in $0.5 \mathrm{M} \mathrm{K}_{2} \mathrm{SO}_{4}+0.1 \mathrm{M}$ Britton-Robinson buffer $-\mathrm{pH} 0.82$.

Figure 4.3.5. 4 Voltammograms at multiple scan rates in $0.5 \mathrm{M} \mathrm{K}_{2} \mathrm{SO}_{4}+0.1 \mathrm{M}$ Britton-Robinson buffer - $\mathrm{pH} 4.01$.

Figure 4.3.5. 5 Voltammograms at multiple scan rates in $0.5 \mathrm{M} \mathrm{K}_{2} \mathrm{SO}_{4}+0.1 \mathrm{M}$ Britton-Robinson buffer - pH 5.89.

Figure 4.3.5. 6 Voltammograms at multiple scan rates in $0.5 \mathrm{M} \mathrm{K}_{2} \mathrm{SO}_{4}+0.1 \mathrm{M}$ Britton-Robinson buffer - $\mathrm{pH} 8.08$.

Figure 4.3.5. 7 Voltammograms at multiple scan rates in $0.5 \mathrm{M} \mathrm{K}_{2} \mathrm{SO}_{4}+0.1 \mathrm{M}$ Britton-Robinson buffer - $\mathrm{pH} 10.50$. 


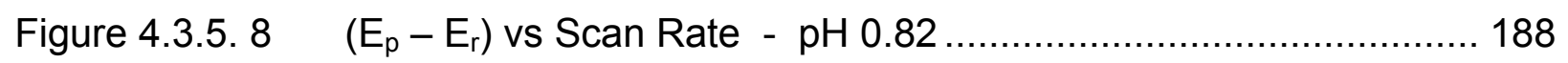

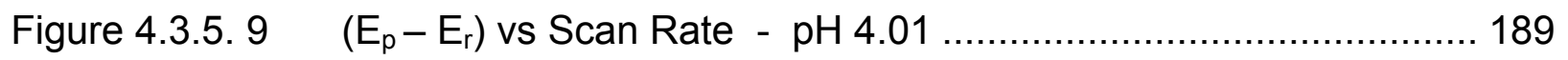

Figure 4.3.5. $10 \quad\left(E_{p}-E_{r}\right)$ vs Scan Rate - pH 5.89 ..................................... 190

Figure 4.3.5. $11 \quad\left(E_{p}-E_{r}\right)$ vs Scan Rate - pH 8.02 …................................. 191

Figure 4.3.5. $12 \quad\left(E_{p}-E_{r}\right)$ vs Scan Rate - pH 10.50 ................................... 191

Figure 4.3.5. $13 \quad \log \mathrm{k}_{\mathrm{s}, \mathrm{app}} \mathrm{vs} \mathrm{pH}$ for $\left[\mathrm{Os}(\mathrm{bpy})_{2}(4-\mathrm{AMP}) \mathrm{OH}\right]_{2}{ }^{2+}$ attached to a $50 / 50$ $\mathrm{HS}\left(\mathrm{CH}_{2}\right)_{12} \mathrm{OH} / \mathrm{HS}\left(\mathrm{CH}_{2}\right)_{15} \mathrm{COOH}$ Monolayer $-\eta=0$ Analysis

Figure 4.3.5. $14 \quad \log \mathrm{k}_{\mathrm{s}}$ vs $\mathrm{pH}$ for $\left[\mathrm{Os}(\mathrm{bpy})_{2}(4-\mathrm{AMP}) \mathrm{OH}_{2}{ }_{2}{ }^{2+}\right.$ attached to a $50 / 50$ $\mathrm{HS}\left(\mathrm{CH}_{2}\right)_{12} \mathrm{OH} / \mathrm{HS}\left(\mathrm{CH}_{2}\right)_{15} \mathrm{COOH}$ Monolayer - Tafel Analysis............................. 195

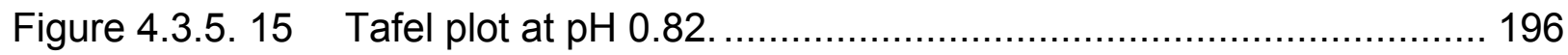

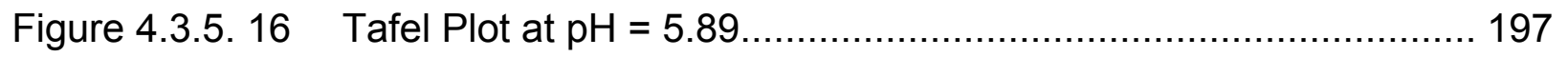

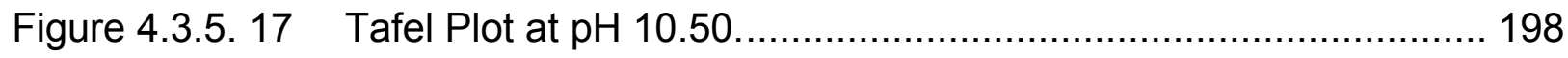

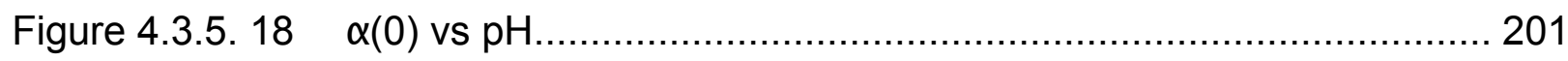




\section{List of Tables}

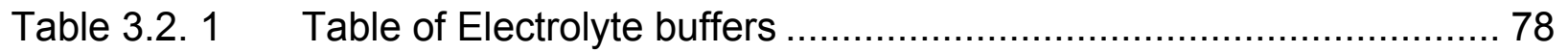

Table 3.4. 1 Apparent Transfer Coefficient vs $\mathrm{pH}$........................................ 100

Table 3.5. 1 Standard Rate Constants vs chain lengths for two redox Centers ... 106

Table 4.1. 1 Formal Potential of $\left[\mathrm{Os}\left(\mathrm{L}_{5}\right) \mathrm{Cl}\right]^{+}$attached to a gold electrode........... 110

Table 4.3.2. 1 Standard Rate Constants for [Os(tpy-o-SAM)(bpy)Cl] ${ }^{+/ 2+} \ldots \ldots \ldots \ldots . . . .171$

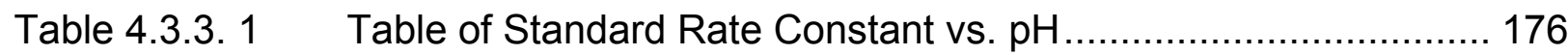

Table 4.3.5. 1 Reorganization Energy vs $\mathrm{pH}$........................................... 199

Table 4.3.5. 2 Calculation of $\lambda$ using Alternate analysis for $\alpha(0) \ldots \ldots \ldots \ldots \ldots \ldots \ldots . \ldots \ldots 202$ 


\section{Table of Abbreviations}

$\begin{array}{ll}\text { PCET } & \text { proton coupled electron transfer } \\ \text { 1e1H } & \text { 1-electron, 1-proton } \\ \text { DOS } & \text { density-of-states } \\ \text { SAM } & \text { self-assembled monolayer } \\ \text { LIA } & \text { lock-in amplifier } \\ \text { WKG } & \text { working electrode } \\ \text { CTR } & \text { counter electrode } \\ \text { REF } & \text { reference electrode } \\ \text { IrREF } & \text { iridium quasi-reference electrode } \\ \text { SCE } & \text { saturated calomel reference electrode } \\ \text { DC } & \text { direct current } \\ \text { AC } & \text { alternating current } \\ \text { FWHM } & \text { full width at half maximum (peak half-width) } \\ \text { CV } & \text { cyclic voltammogram } \\ \text { NMR } & \text { nuclear magnetic resonance } \\ \text { TMAH } & \text { tetramethylammonium hydroxide } \\ \text { UV-VIS } & \text { ultraviolet-visible } \\ \text { TLC } & \text { thin-layer chromatography } \\ \text { DMF } & \text { dimethyl formamide } \\ \text { DMSO } & \text { dimethyl sulfoxide } \\ \text { TFMS } & \text { trifluoromethylsulfonic acid } \\ \text { DCC } & \mathrm{N}, N \text { '-dicyclohexyl-carbodiimide } \\ \text { EDC } & \text { 1-ethyl-3(3-dimethylaminopropyl)-carbodiimide }\end{array}$




\section{Table of Symbols}

\begin{tabular}{|c|c|}
\hline$e^{-}$ & electron \\
\hline $\mathrm{O}$ & oxidized species \\
\hline $\mathrm{R}$ & reduced species \\
\hline $\mathrm{k}_{\mathrm{a}}$ & anodic rate constant in free solution $(\mathrm{cm} / \mathrm{s})$ \\
\hline $\mathrm{k}_{\mathrm{c}}$ & cathodic rate constant in free solution $(\mathrm{cm} / \mathrm{s})$ \\
\hline$C_{x}(0, t)$ & $\begin{array}{l}\text { concentration of species } x \text { at the electrode surface as a function of } \\
\text { time }\left(\mathrm{mol} / \mathrm{cm}^{3}\right)\end{array}$ \\
\hline $\mathrm{v}$ & overall reaction rate \\
\hline $\mathrm{i}$ & current \\
\hline $\mathrm{F}$ & Faraday's constant \\
\hline A & electrode area \\
\hline Z & pre-exponential factor \\
\hline $\mathrm{R}$ & molar gas constant \\
\hline$\Delta \mathrm{G}_{\mathrm{X}}^{\ddagger}$ & free energy of activation for species $X$ \\
\hline$\Delta \mathrm{G}_{0, \mathrm{X}} \neq$ & standard activation energy for species $X$ \\
\hline $\mathrm{T}$ & temperature $\left({ }^{\circ} \mathrm{K}\right)$ \\
\hline $\begin{array}{l}E_{e} \\
F^{0}\end{array}$ & $\begin{array}{l}\text { equilibrium potential } \\
\text { formal notential }\end{array}$ \\
\hline $\mathrm{C}_{\mathrm{X}}{ }^{*}$ & concentration of species $X$ at equilibrium \\
\hline$\alpha$ & cathodic transfer coefficient \\
\hline$\beta$ & anodic transfer coefficient \\
\hline $\mathrm{k}_{\mathrm{X}, 0}$ & rate constant at equilibrium (net current is zero) for branch $X$ \\
\hline$\eta$ & overpotential \\
\hline $\mathrm{kx}_{\mathrm{x}}^{0}$ & standard rate constant for branch $\mathrm{X}$ \\
\hline $\mathrm{k}_{\mathrm{s}}^{0}$ & standard rate constant \\
\hline $\mathrm{i}_{0}$ & exchange current \\
\hline a & empirical Tafel constant \\
\hline$b$ & empirical Tafel constant \\
\hline$\lambda$ & total reorganization energy \\
\hline$\lambda_{\text {is }}$ & Inner-sphere reorganization energy \\
\hline$\lambda_{\text {os }}$ & Outer-sphere reorganization energy \\
\hline$\Delta \mathrm{e}$ & charge transferred from electrolyte to redox couple \\
\hline $\mathrm{D}_{\mathrm{op}}$ & optical dielectric constant \\
\hline$D_{s}$ & static dielectric constant \\
\hline$a_{r}$ & mean radius of molecule \\
\hline$r$ & distance to electrode \\
\hline V & electronic coupling factor \\
\hline $\mathrm{D}(\varepsilon, \lambda, \eta)$ & density of electronic states of the complex \\
\hline
\end{tabular}


$f(\varepsilon) \quad$ density of electronic states energy at the Fermi level on the electrode

$\varepsilon$

$k^{0}$

$\beta$

$n$

$\Gamma_{X}(t)$

$\mathrm{eH}$

$\mathrm{He}$

$\mathrm{E}_{\mathrm{r}}$

$\mathrm{K}_{\mathrm{a}}$

$k_{\text {app }}$

$\mathrm{k}_{\mathrm{s}, \mathrm{app}}$

$\alpha_{X, \text { app }}$

$k_{x}(\eta)$

$\alpha(0)$

$\Psi$

$i_{\text {ch,tot }}$

U

$\mathrm{Q}_{\text {tot }}$

$E_{p, X}$

$\mathrm{D}_{0}$

$\mathrm{n}$

$f(\eta)$

$f_{\text {Nernst }}(\eta)$

$\mathrm{i}_{\mathrm{f}}(\mathrm{\eta})$

k(50\%)

$\mathrm{R}_{\mathrm{u}}$

$\mathrm{R}_{\Omega}$

$\mathrm{C}_{\text {dl }}$

$Z_{f}$ energy level where electron transfer occurs

rate of electron transfer with no attached $\mathrm{CH}_{2}$ 's

Tunneling parameter

number of methylene units in monolayer

Surface coverage of attached species $X\left(\mathrm{~mol} / \mathrm{cm}^{2}\right)$

electron-proton transfer path

proton-electron transfer path

apparent formal potential

acid disassociation constant

apparent rate

apparent standard rate constant

apparent transfer coefficient for branch $X$

rate of branch $X$ at overpotential, $\eta$

transfer coefficient at zero overpotential

dimensionless current

total charging current

scan rate

total charge of adsorbed species on electrode

peak potential of branch $X$

solution diffusion coefficient

number of electrons transferred

actual fraction of reactant charge remaining

theoretical fraction of reactant charge remaining

faradaic current at overpotential, $\eta$

rate from Tafel analysis at $50 \%$ conversion

uncompensated resistance

compensated resistance

double layer capacitance

faradaic impedance 


\section{Introduction}

Proton Coupled Electron Transfer (PCET) reactions play a vital role in a variety of biological and chemical processes. Prior work by E. Laviron developed the theory of proton coupled electron transfer kinetics based on a stepwise mechanism with the transfer coefficient for electron transfer remaining constant at all overpotentials. The present work explores the consequences of a potential-dependent transfer coefficient as applied to redox couples attached to electrodes. Specifically, this work focuses on the 1-electron, 1-proton $(1 \mathrm{e} 1 \mathrm{H})$ system. Derivation of the potential-dependence of the transfer coefficient comes from the Marcus density-of-states model of heterogeneous electron transfer. Two 1e1H systems are examined. The first chapter of this work provides a background on the theoretical aspects of electron transfer of a $1 \mathrm{e} 1 \mathrm{H}$ system. Derivation of the $1 \mathrm{e} 1 \mathrm{H}$ system by Finklea incorporates the Marcus DOS model of electron transfer into Laviron's derivations for proton-coupled electron transfer as applied to electrode-attached redox couples. The second chapter describes the instrumentation used in this project. General experimental methods such as electrode preparation, monolayer deposition and characterization, as well as methods for thermodynamic and kinetic analysis are discussed. The third chapter focuses on the galvinol/galvinoxyl system. Galvinol is an organic redox couple that forms a stable free radical, galvinoxyl, and exhibits $1 \mathrm{e} 1 \mathrm{H}$ behavior. The thermodynamics and kinetics of a galvinol-terminated alkanethiol, adorbed to a gold electrode, are explored. The fourth chapter describes the thermodynamics and kinetics of an inorganic $1 \mathrm{e} 1 \mathrm{H}$ redox couple, 
$\left[\mathrm{Os}(\mathrm{bpy})_{2}(4-\mathrm{AMP}) \mathrm{OH}_{2}\right]^{2+}$, attached to an alkanethol that is adsorbed to a gold electrode. Results show that both the galvinol and the osmium redox couples behave as predicted by the thermodynamic model. However, for both systems, experimental results deviate significantly from the kinetic model. Chapter 5 addresses the clear deviations of the data from theoretical predictions and discusses the future direction of the project. 


\section{Chapter 1 Introduction to Electron Transfer Theory}

\subsection{Electron transfer at an electrode interface in solution: The Butler-Volmer} treatment

The simplest form of an electrochemical electron transfer reaction typically occurs via an outer sphere mechanism. Only one electron is transferred and the reactants are not specifically adsorbed to the electrode. The following are two common examples of such a reaction:

$$
\begin{gathered}
{\left[\mathrm{Ru}\left(\mathrm{NH}_{3}\right)_{6}\right]^{3+}+\mathrm{e}^{-} \rightleftharpoons\left[\mathrm{Ru}\left(\mathrm{NH}_{3}\right)_{6}\right]^{2+}} \\
\text { ferrocenium }{ }^{+}+\mathrm{e}^{-} \rightleftharpoons \text { ferrocene }
\end{gathered}
$$

Electron transfer occurs to an oxidized species, designated as $\mathrm{O}$, to form a reduced species, designated as $\mathrm{R}$ (or vice versa).

$$
\mathrm{O}+\mathrm{e}^{-} \underset{\mathrm{k}_{\mathrm{a}}}{\stackrel{\mathrm{k}_{\mathrm{c}}}{\rightleftharpoons}} \mathrm{R}
$$

Using standard rate law theory, assuming a first order reaction in free solution, the rate of each branch of the reaction can be defined as:

$$
\begin{aligned}
& \text { reduction rate }=\text { cathodic rate }=\mathrm{k}_{\mathrm{c}} \mathrm{C}_{\mathrm{O}}(0, \mathrm{t}) \\
& \text { oxidation rate }=\text { anodic rate }=\mathrm{k}_{\mathrm{a}} \mathrm{C}_{\mathrm{R}}(0, \mathrm{t})
\end{aligned}
$$


where $k_{c}$ and $k_{a}$ are standard rate constants $(\mathrm{cm} / \mathrm{s})$, and $C_{O}(0, t)$ and $C_{R}(0, t)$ are concentrations at the surface of the electrode $\left(\mathrm{mol} / \mathrm{cm}^{3}\right)$ as a function of time ${ }^{1}$. Note that the rate is related to the area of the electrode. The overall reaction rate can be defined as

$$
\text { overall rate }=v=\mathrm{k}_{\mathrm{c}} \mathrm{C}_{\mathrm{O}}(0, \mathrm{t})-\mathrm{k}_{\mathrm{a}} \mathrm{C}_{\mathrm{R}}(0, \mathrm{t})
$$

Cathodic currents are positive and anodic currents are negative. Essentially, the overall rate is analogous to the flux of $\mathrm{O}$ at the electrode surface and is proportional to the net current

$$
\mathrm{i}=\mathrm{FA} v=\mathrm{FA}\left(\mathrm{k}_{\mathrm{c}} \mathrm{C}_{\mathrm{O}}(0, \mathrm{t})-\mathrm{k}_{\mathrm{a}} \mathrm{C}_{\mathrm{R}}(0, \mathrm{t})\right)
$$

where $\mathrm{F}$ is Faraday's constant and $\mathrm{A}$ is the area of the electrode. Application of the Arrhenius equation for temperature dependence yield the following expressions for the rate constant

$$
\begin{aligned}
& \mathrm{k}_{\mathrm{c}}=\mathrm{Z}_{\mathrm{c}} \mathrm{e}^{\frac{-\Delta \mathrm{G}_{\mathrm{c}}^{\ddagger}}{\mathrm{RT}}} \\
& \mathrm{k}_{\mathrm{a}}=\mathrm{Z}_{\mathrm{a}} \mathrm{e}^{\frac{-\Delta \mathrm{G}_{\mathrm{a}}^{\ddagger}}{\mathrm{RT}}}
\end{aligned}
$$

where $\Delta G^{\ddagger}$ 's are free energies of activation, $R$ is the molar gas constant, and $T$ is temperature, and $Z$ is the pre-exponential factor. 
At equilibrium, the anodic and cathodic standard rate constants are equal which results in a net current of zero. Manipulation of eqs (1.1.6) and (1.1.7) with subsequent substitution into eq (1.1.5) results in

$$
\ln \frac{\mathrm{C}_{\mathrm{O}}(0, \mathrm{t})}{\mathrm{C}_{\mathrm{R}}(0, \mathrm{t})}=\ln \frac{\mathrm{k}_{\mathrm{a}}}{\mathrm{k}_{\mathrm{c}}}=\frac{\Delta \mathrm{G}_{\mathrm{c}}^{\ddagger}-\Delta \mathrm{G}_{\mathrm{a}}^{\ddagger}}{\mathrm{RT}}
$$

The relationship between the ratio of reactant and product is defined in the Nernst equation ${ }^{1}$ :

$$
E_{e}=E^{o}-\frac{R T}{F} \ln \frac{C_{R}(0, t)}{C_{O}(0, t)}
$$

where $E_{e}$ is the equilibrium (reversible) potential and $E^{0}$ is the formal potential of the system. Rearrangement of the Nernst equation leads to

$$
\ln \frac{\mathrm{C}_{\mathrm{O}}^{*}}{\mathrm{C}_{\mathrm{R}}^{*}}=\frac{\mathrm{F}\left(\mathrm{E}_{\mathrm{e}}-\mathrm{E}^{\mathrm{o}}\right)}{\mathrm{RT}}
$$

(where $\mathrm{C}_{\mathrm{O}}{ }^{*}$ and $\mathrm{C}_{\mathrm{R}}{ }^{*}$ are concentrations at equilibrium in the bulk solution) and substitution into eq (1.1.8)allows direct correlation between the potential and the Gibbs free energy of the system:

$$
\Delta \mathrm{G}_{\mathrm{c}}^{\ddagger}-\Delta \mathrm{G}_{\mathrm{a}}^{\ddagger}=\mathrm{F}\left(\mathrm{E}_{\mathrm{e}}-\mathrm{E}^{\circ}\right)
$$

This idea is a common one in the context of chemical kinetics and is easily described in a free energy diagram of a simple electron transfer process, shown graphically in Figure 1.1.1. By the definition of eq (1.1.11), when the equilibrium potential is equal to 


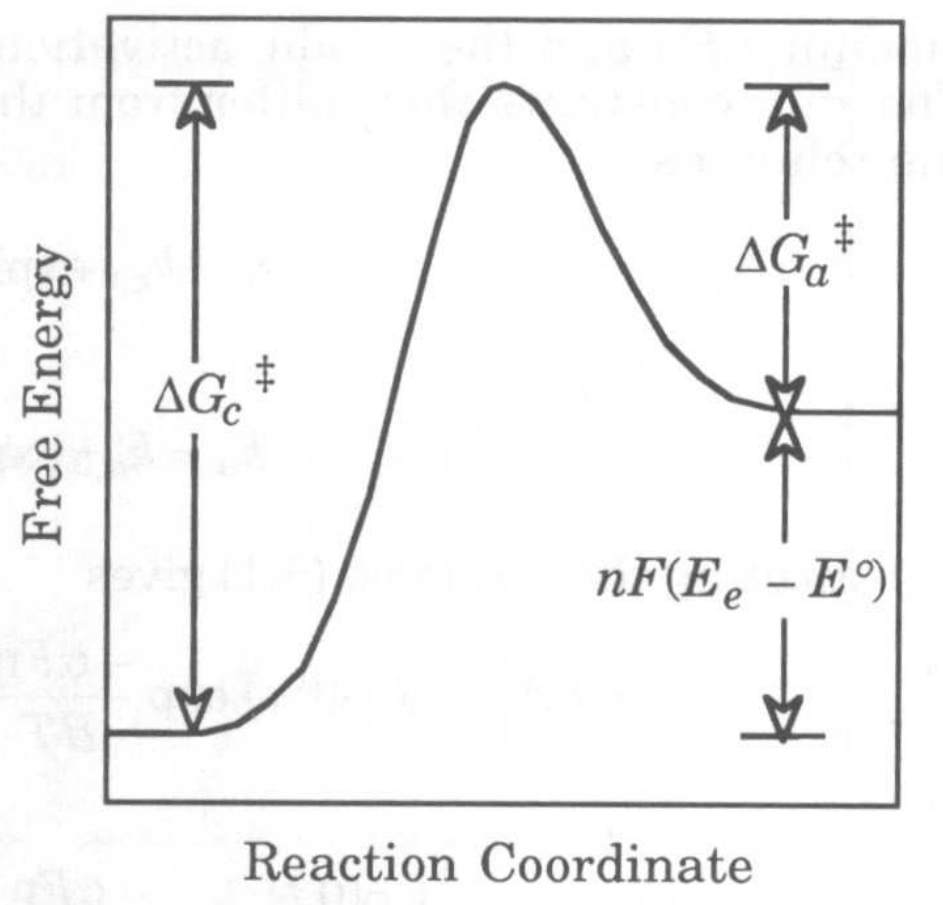

Figure 1.1.1 Free energy diagram of an Electron Transfer Process ${ }^{1}$ 
the formal potential, the free energies of activation for each side of the reaction are equal, and this is equal to the standard activation energy for the process. This is a special case, where $\mathrm{C}_{O}{ }^{*}=\mathrm{C}_{\mathrm{R}}{ }^{*}$. Then,

$$
\Delta \mathrm{G}_{\mathrm{c}}^{\ddagger}=\Delta \mathrm{G}_{\mathrm{a}}^{\ddagger}=\Delta \mathrm{G}_{0}^{\ddagger} \quad\left(\mathrm{E}_{\mathrm{e}}-\mathrm{E}^{\circ}=0\right)
$$

When a more negative potential is applied, the energy of the transferring electron is increased which shifts the oxidant curve to a higher energy, as seen in Figure 1.1.2. The end result is a net cathodic current, because the oxidative barrier is higher, and the reductive barrier is lower than it is at equilibrium. The opposite occurs when a more positive potential is applied. The oxidant curve shifts to a potential lower than equilibrium, which causes a net anodic current. With this in mind, a phenomenological approach can be used to estimate the effect potential will have on the activation free energy of a system. As seen in the following equations, the free activation energy of both the cathodic and anodic branch of the process can be calculated knowing $\Delta \mathrm{G}_{0}{ }^{\ddagger}$ and $E_{e}-E^{0}$

$$
\begin{gathered}
\Delta \mathrm{G}_{\mathrm{c}}^{\ddagger}=\Delta \mathrm{G}_{0, \mathrm{c}}^{\ddagger}+\alpha \mathrm{F}\left(\mathrm{E}_{\mathrm{e}}-\mathrm{E}^{\mathrm{o}}\right) \\
\Delta \mathrm{G}_{\mathrm{a}}^{\ddagger}=\Delta \mathrm{G}_{0, \mathrm{a}}^{\ddagger}-\beta \mathrm{F}\left(\mathrm{E}_{\mathrm{e}}-\mathrm{E}^{\mathrm{o}}\right)
\end{gathered}
$$

where $\alpha$ and $\beta$ are the cathodic and anodic transfer coefficients, respectively, and $\beta=$ $(1-\alpha)^{1}$. This relationship is also seen in Figure 1.1.2. The role of the transfer coefficients will be discussed in detail later.

By substitiuting eqs (1.1.13) and (1.1.14) into the Arrhenius derivation of the standard rate constant, eqs (1.1.6) and (1.1.7), we get 


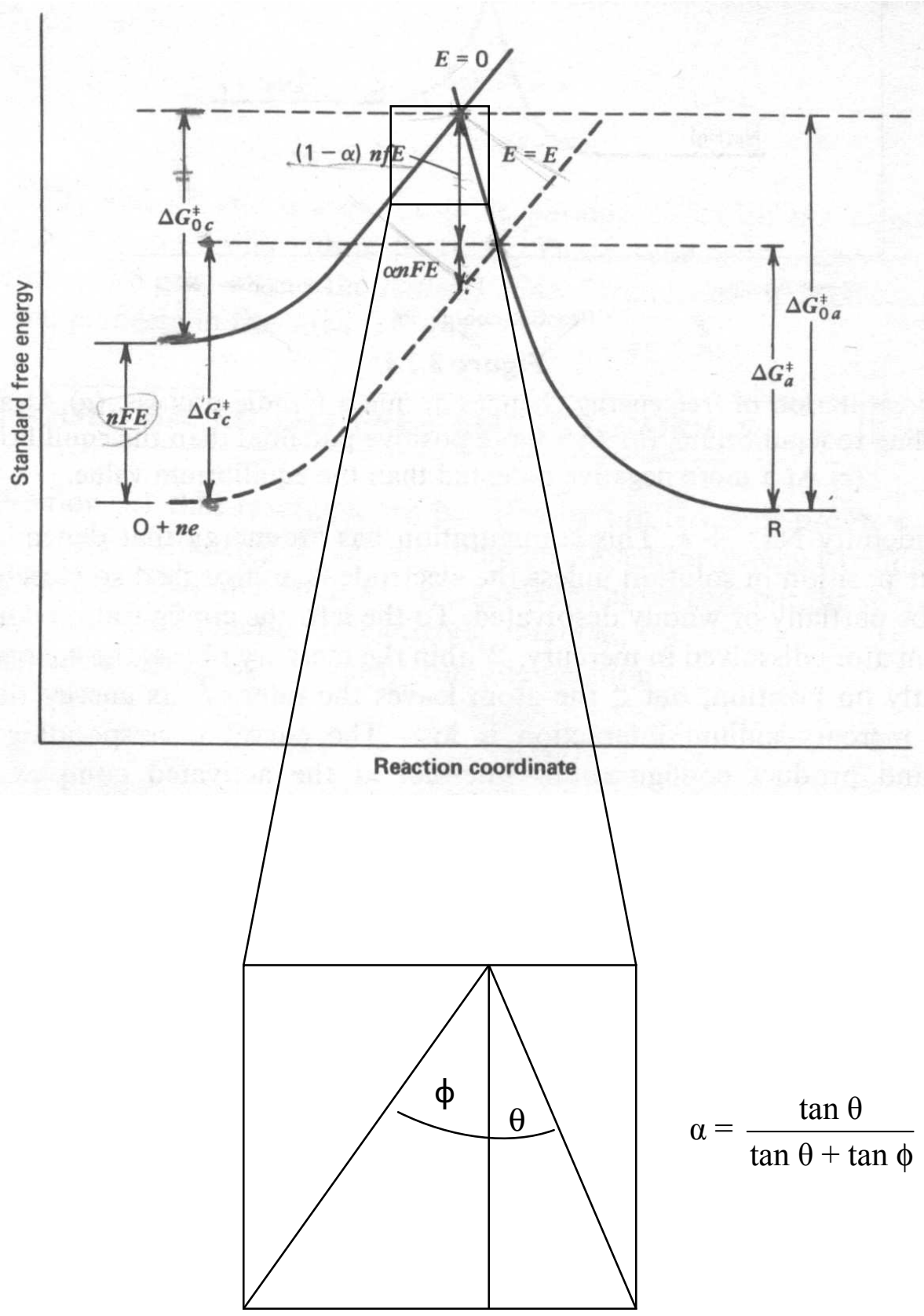

Figure 1.1.2 The effect of electrode potential on a Reaction Coordinate diagram ${ }^{2}$. The dashed curve represents initial equilibrium conditions, and the solid curve represents conditions after a negative potential (with respect to the formal potential) is applied. The expanded section shows the relationship between $\alpha$ and the shape of the Reaction Coordinate diagram. 


$$
\begin{gathered}
\mathrm{k}_{\mathrm{c}, 0}=\mathrm{k}_{0} \mathrm{e}^{\frac{-\alpha \mathrm{F}\left(\mathrm{E}_{\mathrm{e}}-\mathrm{E}^{\mathrm{o}}\right)}{\mathrm{RT}}} \\
\mathrm{k}_{\mathrm{a}, 0}=\mathrm{k}_{0} \mathrm{e}^{\frac{\beta \mathrm{F}\left(\mathrm{E}_{\mathrm{e}}-\mathrm{E}^{\mathrm{o}}\right)}{\mathrm{RT}}} \\
\text { where } \mathrm{k}_{0}=\mathrm{Ze}^{\frac{-\Delta \mathrm{G}_{0}^{\ddagger}}{\mathrm{RT}}}
\end{gathered}
$$

The above derivation describes the system with a net current of zero. When a net current flows, the cell is not at equilibrium. The corresponding deviation of the cell potential from the equilibrium potential is called the applied overpotential.

$$
\eta=E-E_{e}
$$

If we assume that eqs (1.1.13) and (1.1.14) are valid under non-equilibrium conditions, then eq (1.1.18) can be substituted into eqs (1.1.15) and (1.1.16). It can be seen that the anodic and cathodic rate constants will deviate from their respective rate as a function of overpotential according to the following expressions

$$
\begin{aligned}
& \mathrm{k}_{\mathrm{c}}=\mathrm{k}_{\mathrm{c}}^{0} \mathrm{e}^{\frac{-\alpha \mathrm{FT}}{\mathrm{RT}}} \\
& \mathrm{k}_{\mathrm{a}}=\mathrm{k}_{\mathrm{a}}^{0} \mathrm{e}^{\frac{\beta \mathrm{F} \eta}{\mathrm{RT}}}
\end{aligned}
$$

Considering a special case where $C_{O}{ }^{*}=C_{R}{ }^{*}$, it becomes apparent from eq (1.1.9) that $E_{e}$ $=E^{0}$ and $k_{c} C_{O}{ }^{*}=k_{a} C_{R}{ }^{*}$. This implies that $k_{a}=k_{c}$. This rate under these reversible equilibrium conditions is defined as the standard rate $k_{s}{ }^{0}$. Substitution of these into eqs (1.1.19) and (1.1.20) yield

$$
\mathrm{k}_{\mathrm{c}}=\mathrm{k}_{\mathrm{s}}^{0} \mathrm{e}^{\frac{-\alpha \mathrm{F} \eta}{\mathrm{RT}}}
$$




$$
\mathrm{k}_{\mathrm{a}}=\mathrm{k}_{\mathrm{s}}^{0} \mathrm{e}^{\frac{\beta \mathrm{FT} \eta}{\mathrm{RT}}}
$$

Substitution of these into eq (1.1.5) gives

$$
i=\operatorname{FAk}_{\mathrm{s}}^{0}\left[\mathrm{C}_{\mathrm{O}}(0, \mathrm{t}) \mathrm{e}^{\frac{-\alpha \mathrm{F} \eta}{\mathrm{RT}}}-\mathrm{C}_{\mathrm{R}}(0, \mathrm{t}) \mathrm{e}^{\frac{\beta F \eta}{\mathrm{RT}}}\right]
$$

Because the exchange current, $\mathrm{i}_{0}$, is equal to the cathodic current at equilibrium, or opposite the anodic current at equilibrium, then

$$
\mathrm{i}_{0}=\mathrm{FAk}_{\mathrm{s}}^{0} \mathrm{C}_{\mathrm{O}}^{*}=-\mathrm{FAk}_{\mathrm{s}}^{0} \mathrm{C}_{\mathrm{R}}^{*}
$$

where $C_{O}{ }^{*}$ and $C_{R}{ }^{*}$ are used because the system is at equilibrium. Substitution into eq (1.1.23) yields

$$
i=i_{0}\left[\frac{C_{O}(0, t)}{C_{O}^{*}} e^{\frac{-\alpha, F \eta}{R T}}-\frac{C_{R}(0, t)}{C_{R}^{*}} e^{\frac{\beta F \eta}{R T}}\right]
$$

This equation can be simplified further by the assumption that transport of reactant and product is very fast and essentially at equilibrium, where $C(0, t)=C^{*}$, giving

$$
\mathrm{i}=\mathrm{i}_{0}\left[e^{\frac{-\alpha F \eta}{R T}}-e^{\frac{\beta F \eta}{R T}}\right]
$$

This is termed the Butler-Volmer equation ${ }^{2}$. This equation defines the dependence of current on the overpotential. This dependence is best seen by using Tafel analysis. 


\subsection{The Tafel Equation}

Tafel analysis was developed in the early 1900's as a method of relating the potential of an electrode to the current density passing through the electrode. In its simplest form, the Tafel equation is as follows

$$
\log (\mathrm{i})=a+b \eta
$$

where $a$ and $b$ are empirical constants ${ }^{3}$. This equation fit observed data for a number of kinetically controlled electron transfer reactions, such as $\mathrm{O}_{2}$ reduction and $\mathrm{H}^{+}$ reduction $^{3}$. A graphical plot of this equation yields a line of slope b with a y-intercept of a. In order to extract useful information from the plot, the empirical constants must be evaluated. A viable approach at quantifying Tafel analysis uses the Butler-Volmer approach. Equation (1.1.26) includes the contribution of both the anodic and cathodic branches to the net current, i. It is important to note that, at high positive overpotentials, the cathodic contribution (containing $\alpha$ ) is negligible compared to the anodic contribution (containing $\beta$ ). The reverse applies at high negative overpotentials. The consequence of this observation is that the Butler-Volmer equation can be broken down into two equations describing the current along each branch

$$
\mathrm{i}_{\text {cat }}=\mathrm{i}_{0}\left[\mathrm{e}^{\frac{-\alpha \mathrm{F} \eta}{\mathrm{RT}}}\right] \quad \eta<0
$$




$$
\mathrm{i}_{\text {an }}=\mathrm{i}_{0}\left[\mathrm{e}^{\mathrm{\beta F} \eta}\right] \quad \eta>0
$$

Taking the logarithm of these two equations yields

$$
\begin{aligned}
& \log \left(\mathrm{i}_{\text {cat }}\right)=\log \left(\mathrm{i}_{0}\right)-\frac{\alpha \mathrm{F \eta}}{2.3 \mathrm{RT}} \\
& \log \left(\mathrm{i}_{\text {an }}\right)=\log \left(\mathrm{i}_{0}\right)+\frac{\beta F \eta}{2.3 \mathrm{RT}}
\end{aligned}
$$

It can be seen that (1.2.4) and (1.2.5) follow the form of the Tafel equation with $a=$ $\log \left(\mathrm{i}_{0}\right)$ and $b=-\alpha F / 2.3 R T$ for the cathodic branch and $b=\beta F / 2.3 R T$ for the anodic branch. A generic Tafel plot is seen in Figure 1.2.1. Note that typically in a Tafel plot, log lil is plotted versus $\eta$, which allows direct correlation between each branch. It is important to see that a true Tafel plot is linear at intermediate overpotential, but deviates from linearity at large and small overpotentials. At small overpotentials (region a) the assumption that the contribution to the net current is due only to one branch breaks down. In other words, the opposing current becomes significant, yielding net currents that are less than currents predicted by eqs (1.2.2) and (1.2.3)

On the other end of the spectrum, at extreme overpotentials, deviation from the theoretical slope occurs as a consequence of mass transfer. The derivation of the Butler-Volmer equation is based on the assumption that the concentration of analyte at the surface of the electrode is the same as the bulk concentration of analyte in solution. 


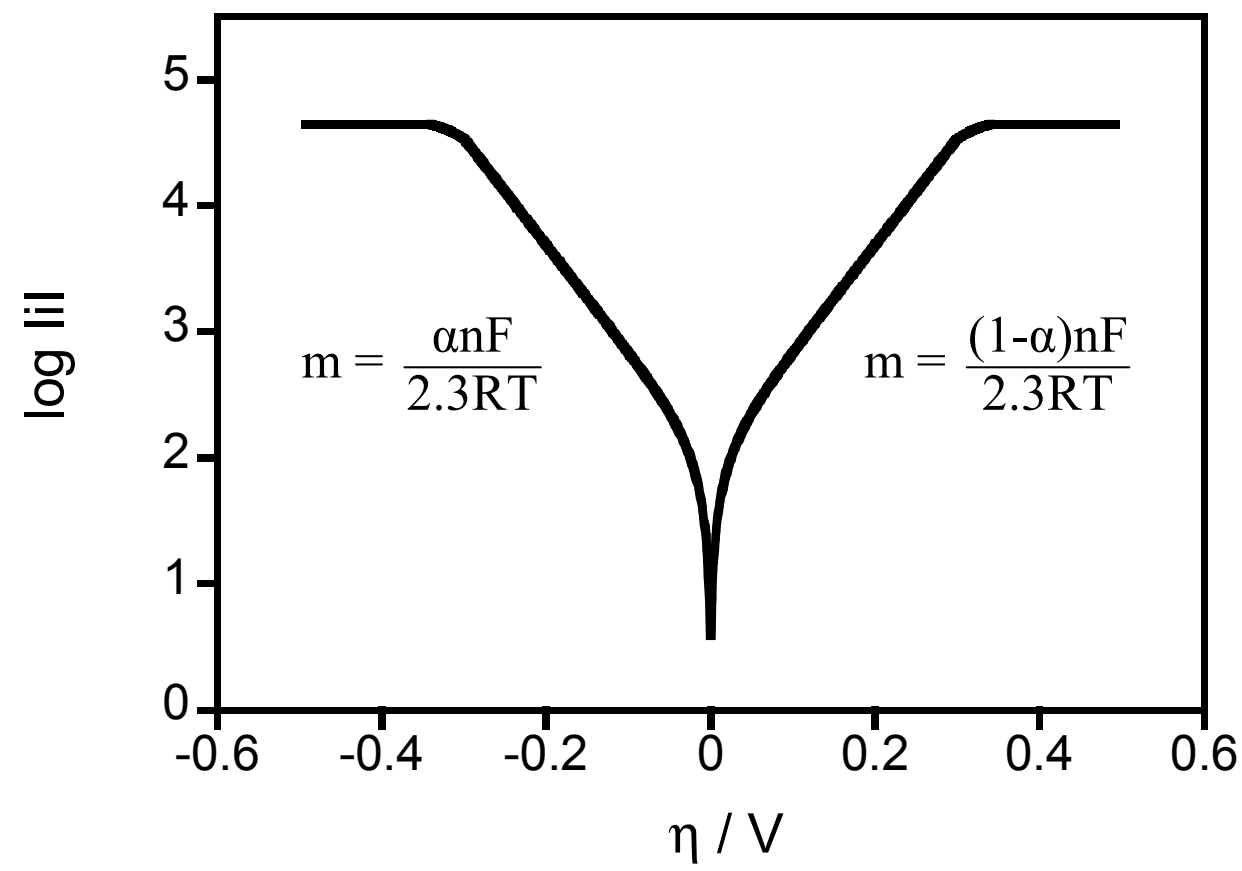

Figure 1.2. 1 General Tafel Plot 
This requires to sufficient mass transfer, which is a function of three modes: diffusion, convection and migration. At regions where the rate of electron transfer approaches the mass transport rate, the overall rate starts to become mass transfer limiting (because the overall rate is limited by the slowest contributor). At extreme potentials, the rate becomes completely mass transfer limited, resulting in a line with a slope of zero. Essentially, the same amount of analyte is oxidized or reduced regardless of the overpotential. Mass Transfer effects can be troublesome in the context of solution electrochemistry. However in the context of this research, mass transfer is of minor consideration, so a more detailed exploration is not needed.

At intermediate overpotentials in a Tafel plot, the rate of electron transfer is much slower than the rate of transport of the analyte to the electrode. Mass transfer effects are minimized, and only one branch significantly contributes to the net current. Overpotentials greater than $0.1 \mathrm{~V}$ typically ensure that there is no back current. This is called the Tafel region, and it is the area in a Tafel plot where the plot is linear.

Tafel Analysis will be discussed further throughout this paper. Two important parameters in the Butler-Volmer equation are the anodic and cathodic transfer coefficients. The next section will discuss the significance of the transfer coefficients. 


\subsection{The Transfer Coefficeint}

Transfer coefficients are introduced qualitatively to separate the contributions of $\Delta G_{c}{ }^{\ddagger}$ and $\Delta G_{a}{ }^{\ddagger}$. According to Bard $^{2}$, "the transfer coefficient is a measure of the symmetry of the energy barrier". Using the free energy/reaction coordinate diagram from earlier, Figure 1.1.2, the transfer coefficient can be qualitatively compared to the angle on each side of the transition $(\Theta$ and $\phi)$. Figure 1.3.1 shows the effect that the value of the transfer coefficient has on the symmetry of the intersection of the free energy curves.

From eqs (1.1.19) and (1.1.20) it can be seen that the values for the transfer coefficient have a direct impact on the apparent rate. It is important to reiterate that the sum of the transfer coefficients equals one

$$
\alpha+\beta=1
$$

In order to show the direct relationship between $\alpha$ and $\beta$, the anodic transfer coefficient will henceforth be referred to as $(1-\alpha)$. Ordinarily, the anodic and cathodic transfer coefficients are equal, and the anodic and cathodic currents respond symmetrically to changes in overpotential. There are exceptions to this rule, and these conditions will be discussed later. 

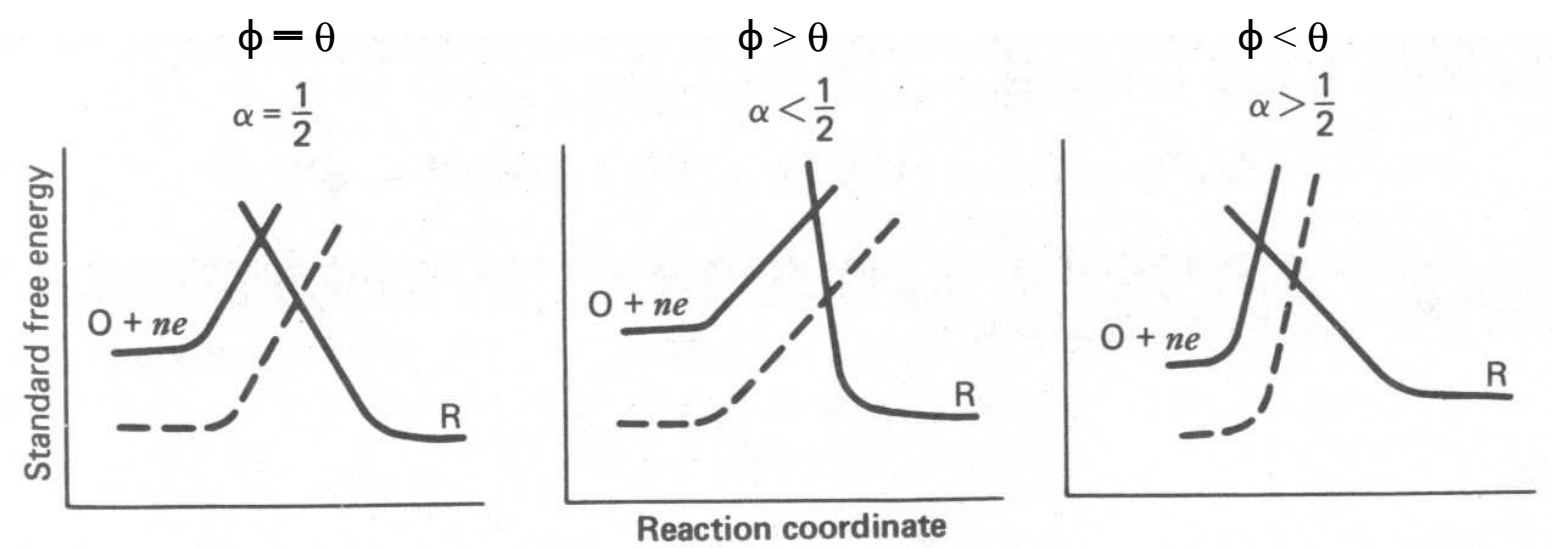

Figure 1.3. 1 Diagram ${ }^{2}$ 


\subsection{Deficiencies of the Butler-Volmer Approach}

The Butler-Volmer approach is successful in providing a generalized theory of electrode kinetics. It describes the apparent rate of a reaction as dependent on only three parameters: the standard rate constant, the transfer coefficient, and the applied overpotential. Unfortunately, Butler-Volmer theory is limited in practice because it is defined in such broad terms. Empirically, while the Butler-Volmer approach worked for some systems, it was shown to be deficient in describing some simple pure 1-electron systems $^{5}$. In order to account for deficiencies in the Butler-Volmer approach, it was necessary to look at electron transfer on a microscopic scale. To accomplish this, a more complicated mathematical model for predicting rates of electron transfer needed to be developed. The most widely accepted model was developed by R.A. Marcus. The model relies heavily on transition-state theory. The Marcus Density of States (DOS) Theory of electron transfer takes into account microscopic events that have an impact on the electron transfer rate.

\subsection{Introduction to DOS Theory}

It is convenient to think of an electron transfer process as a series of elemental steps $^{6}$. A depiction of each step is depicted graphically in Figure 1.5.1. The electron transfer process begins with mass transport of the redox couple to the surface of the

electrode. Specifically, the electroactive reactant travels to the electrode surface, and 


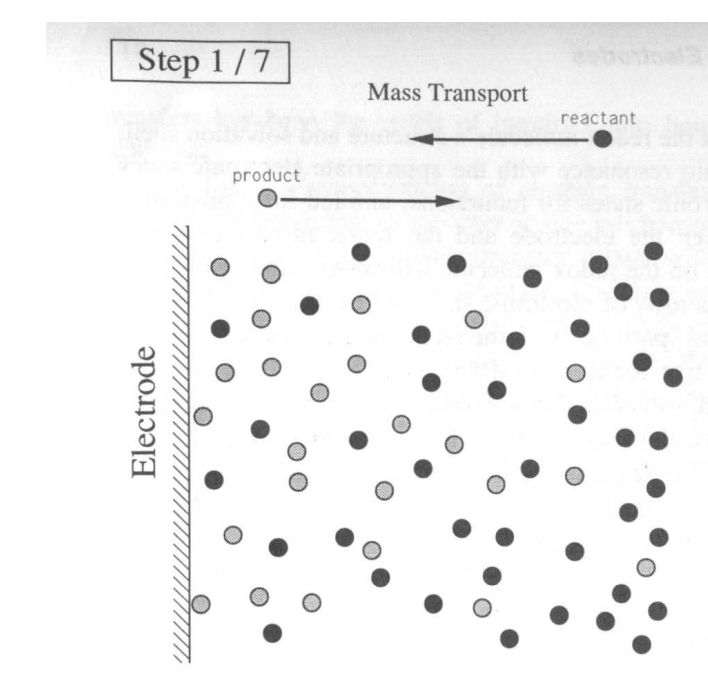

Step $3 / 5$

Thermal Activation and Relaxation

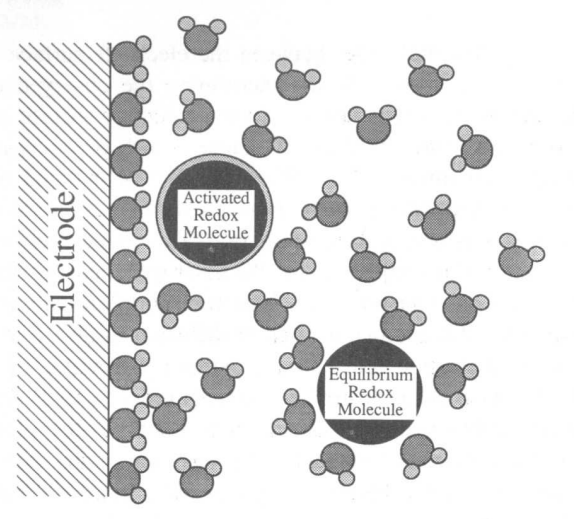

\section{Step 2/6}

Surface Interactions Between Electrode and Redox Molecules

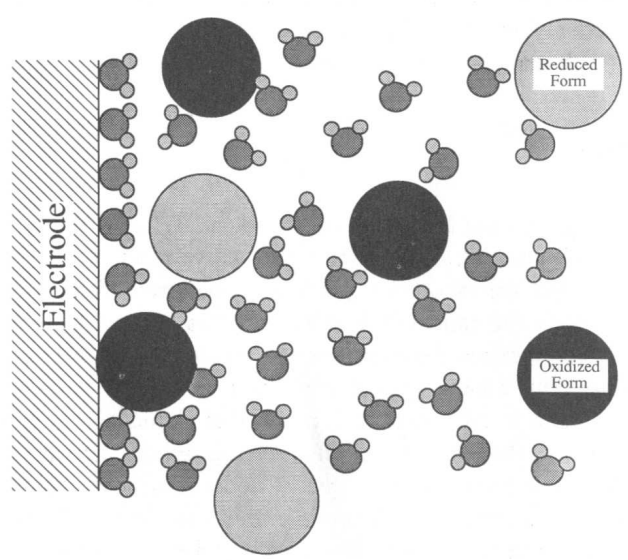

Step 4

Electron Tunneling Between Electrode and Redox Molecule

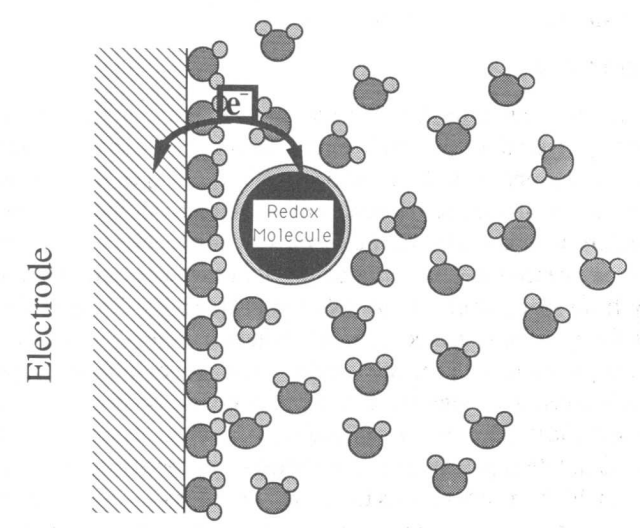

Figure 1.5. 1 Mechanistic Steps in the Electron Transfer Process ${ }^{7}$ 
the product travels away from the electrode surface. The second step involves specific attraction or repulsion of the reactant to the electrode (due to electrostatic and chemical effects). The concentration of reactant near the surface of the electrode can vary dramatically from the concentration of reactant in bulk solution. This coupling between the electrode and the reactant can have a dramatic effect on electron transfer rate. The next step involves the thermal activation of the reactant. In order for electron transfer to occur, there must be an electronic resonance with the appropriate electronic states of the metal electrode and the redox couple. This resonance is achieved through fluctuation of the redox couple's internal conformation and solvation shell. Once the reactant achieves an activated state, electron transfer can occur. An electron will tunnel between the electrode and redox molecule with a rate dependent on the distance of the redox species from the electrode, the orbital orientation of the redox molecule with respect to the electrode, the density of overlapping electronic states, and the nature of the interface between the redox molecule and the electrode ${ }^{5}$. After electron transfer, the redox molecule relaxes from its activated state towards its equilibrium orientation. The new molecule can interact specifically with the electrode at close proximity to the electrode surface. Then it is carried away towards the bulk solution by mass transport. Because of the complexity and sheer number of elementary steps, it becomes important to thoroughly understand each individual step in order to arrive with an accurate mathematical model. The overall rate is dependent upon the rates of each of the individual steps. 


\subsection{Elementary Steps in Marcus DOS Theory}

\section{Mass Transport}

Transport of an analyte from bulk solution to the surface of the electrode is an important concept in the overall determination of electron transfer rate. In an ideal situation, it is desirable to have the rate of transport to greatly exceed the rates of the other elemental steps. This ensures that there is no contribution from mass transport to the overall reaction rate. Unfortunately, this ideal situation is not always achievable. If the rate of mass transport becomes less than the rate of the other elementary steps, the overall reaction rate becomes mass transport limited. As most voltammetric techniques use an unstirred electrolyte, this is typically termed a diffusion-limited rate ${ }^{5}$. Under diffusion-limited conditions, any attempt to understand the other elementary steps is compromised. Therefore in order to get information on the electron transfer process itself, it is necessary to eliminate any contribution of mass transport to the rate. Dealing with mass transfer can be problematic; it will be discussed in detail later, in the context of the present research project.

\section{Electrode/Analyte interaction}

The concentration of analyte near the surface of the electrode is dependent on a number of variables. Electrostatic interactions with the electrode, specific chemical interactions with the electrode surface, and solvent properties all affect the concentration of the analyte near the surface of the electrode and consequently the 
rate of electron transfer. For example, a negatively polarized electrode will attract cations in solution, making the equilibrium near-surface concentration higher than the concentration of the bulk solution. This type of anomaly leads to double-layer effects. There are two types of double layer effects. The first type involves changes in the surface concentration vs bulk solution as described above. The second type involves changes in the driving force for electron transfer due to changes in the local potential, thus $\eta_{\text {actual }} \neq \eta_{\text {applied. }}$. The consequences of double-layer effects occur in the kinetic regime. Kinetically, these changes in double-layer interactions are seen in deviations of the reaction rate. They can lead to broader distributions of reaction rates. Thus, it is helpful to minimize double-layer effects prior to analysis.

\section{Thermal Activation}

In order for electron transfer to occur, the acceptor/donor electronic states of the redox molecule must be in equilibrium with the complementary electronic states of the metal electrode. This means that the energies of the two complementary states must be equal. The Fermi level of the metal electrode is defined as the energy level at the transition of occupied and unoccupied energy states. All states above the Fermi level (higher negative potential) are unoccupied, and all states below the Fermi level are occupied. The energy of the Fermi level is controlled directly by the applied potential of the electrode. This absolute definition of the Fermi level is not completely correct (there is some observed curvature to the transition), but as an approximation, it is sufficient. The energy level of the acceptor/donor states of the analyte is a dynamic 
property and fluctuates around the energy associated with the formal potential of the analyte by changes in the internal structure and outer solvation sphere. Because of these random fluctuations, only those molecules whose energy level is equal to the complementary state in the metal band are considered activated. The energy of the activated complex is typically much higher than the energy of the complex at equilibrium, so the probability of a molecule being in the activated state is low. The energy required to change from the equilibrium structure of one oxidation state to the structure of the other oxidation state without actually transferring an electron is defined as the molecule's reorganization energy, $\lambda$. The reorganization energy of a system is the summation of an inner sphere and outer sphere component.

$$
\lambda=\lambda_{\text {is }}+\lambda_{\text {os }}
$$

The inner sphere reorganization energy is based on the actual physical structure rearrangement associated with the charge transfer. This is a complex feature and can be dependent on the oxidation state of the molecule. The outer sphere contribution is based on the change in the solvent structure as the oxidation state of the redox molecule changes. $\lambda_{\text {os }}$ can be estimated using dielectric continuum theory and is independent of the oxidation state. The outer sphere reorganization energy contribution is described by the following equation

$$
\lambda_{\mathrm{os}}=(\Delta \mathrm{e})^{2}\left(\frac{1}{\mathrm{D}_{\mathrm{op}}}-\frac{1}{\mathrm{D}_{\mathrm{s}}}\right)\left(\frac{1}{2 a_{r}}-\frac{1}{2 r}\right)
$$

where $\Delta e$ is the charge transferred from the electrode to the redox couple, $D_{o p}$ is the optical dielectric constant and $D_{s}$ is the static dielectric constant, $a_{r}$ is the mean radius 
of the molecule, and $r$ is the distance to the electrode $)^{8}$. For a molecule in water with an approximate mean radius of $2 \AA$, located $20 \AA$ from the electrode, $\lambda_{\text {os }}=0.90 \mathrm{eV}^{9}$.

\section{Electron Transfer Step}

The electron transfer step is complex, involving a number of variables.

Thermodynamically, the barrier to electron transfer can be approximately represented by Figure 1.1.1. As the focus of this project, details on the electron transfer step will be provided later.

\section{Post Electron Transfer Steps}

An important concept in understanding electron transfer using the DOS model is that electron transfer occurs after activation and before relaxation of the molecule takes place. This is simply an application of the Frank-Condon principle which states that "the transition from one energy state to another is so rapid that the nuclei of the atoms involved can be considered to be stationary during the transition ${ }^{10}$." Or simply put, the time frame of electronic transitions is much faster than vibrational transitions. This explains why electron transfer is a discrete step from activation and deactivation, or relaxation, of the redox couple.

After electron transfer, the new analyte then relaxes down to its equilibrium state (because the probability of the redox couple staying in the activated state is very low). After relaxation, the species then may have specific interaction with the electrode. 
Concentration changes eventually cause mass transport away from the electrode to the bulk solution.

\subsection{Derivation of Rate Expressions of DOS Theory - The Marcus Approach}

Marcus' theoretical approach to describing the rate of electron transfer for a simple system is based on the following standard equation for reaction rate

$$
\mathrm{k}=\mathrm{VD}(\varepsilon, \lambda, \eta) \mathrm{f}(\varepsilon)
$$

where $f(\varepsilon)$ is the density of the electronic-state distribution of the electrode at the Fermi level, $V$ is the electronic coupling factor, and $D(\varepsilon, \lambda, \eta)$ is the density of acceptor/donor states. It is convenient to use the Fermi energy of the metal electrode as the reference energy. $D(\varepsilon, \lambda, \eta)$ is defined as follows ${ }^{8}$ :

$$
\mathrm{D}(\varepsilon, \lambda, \eta)=\frac{\mathrm{e}^{\frac{-(\varepsilon \pm \lambda-\mathrm{e} \eta)^{2}}{4 \lambda \mathrm{kT}}}}{(4 \pi \lambda \mathrm{kT})^{1 / 2}}
$$

where $\varepsilon$ is the energy at which electron transfer takes place with respect to the Fermi energy level $\left(\varepsilon=E-E_{f}\right), \lambda$ is the reorganization energy of the process $(-\lambda$ for reduction and $+\lambda$ for oxidation), and $\eta$ is the applied overpotential $\left(E-E^{0}\right)$. This equation now relates the density of states to two measurable variables, reorganization energy and overpotential. The density of the occupied electronic states on the electrode, $f(\varepsilon)$, is defined as: 


$$
f(\varepsilon)_{c}=\frac{1}{1+e^{\frac{\varepsilon}{k T}}}
$$

for reduction, and

$$
\mathrm{f}(\varepsilon)_{\mathrm{a}}=\frac{\mathrm{e}^{\frac{\varepsilon}{\mathrm{kT}}}}{1+\mathrm{e}^{\frac{\varepsilon}{\mathrm{kT}}}}
$$

for oxidation. Equation (1.7.1) is a description of the rate of electron transfer at the Fermi level. However, some electron transfer does occur away from the Fermi level. In order to get a complete rate approximation, one must look at the contribution to the rate over all energy levels. This can be accomplished by integrating equation (1.7.1) over all energy levels

$$
\mathrm{k}=\mathrm{V} \int_{-\infty}^{\infty} \mathrm{D}(\varepsilon, \lambda, \eta) \mathrm{f}(\varepsilon) \mathrm{d} \varepsilon
$$

Note that the electronic coupling contribution is carried outside the integration because it is assumed to be independent of the Fermi energy of the electrode. Qualitatively, the rate of electron transfer is proportional to the amount of overlap between the acceptor states of the complex and the donor states of electrode (or vice versa). Figure 1.7.1 is representative of the reduction of an oxidized species. The separation of the distribution peak from the formal potential is equal to the reorganization energy of each branch. Also, the half-width of the distribution is proportional to the reorganization energy, with lower $\lambda$ 's resulting in more narrow distributions. In Figure 1.7.1.a, because the Fermi level is significantly lower than the formal potential (the potential is held sufficiently positive), there is only minor overlap between the filled states 


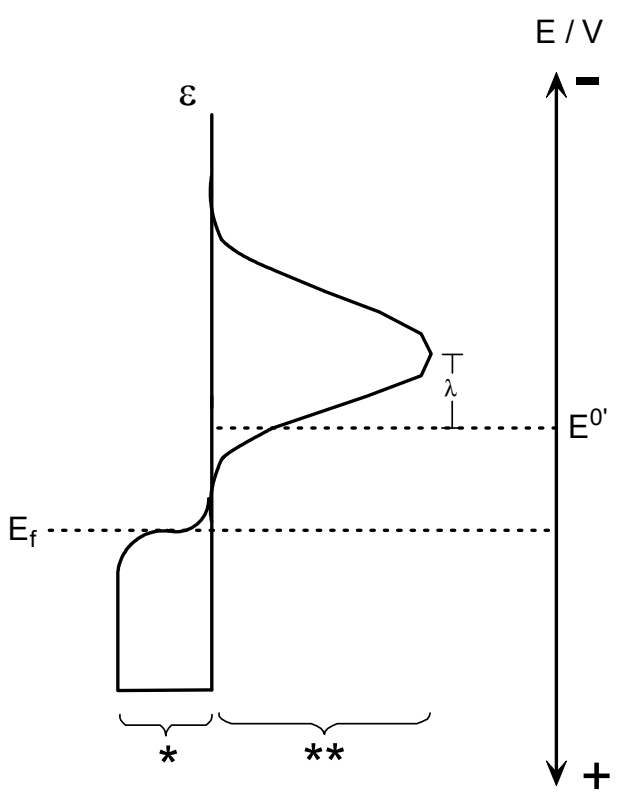

a.

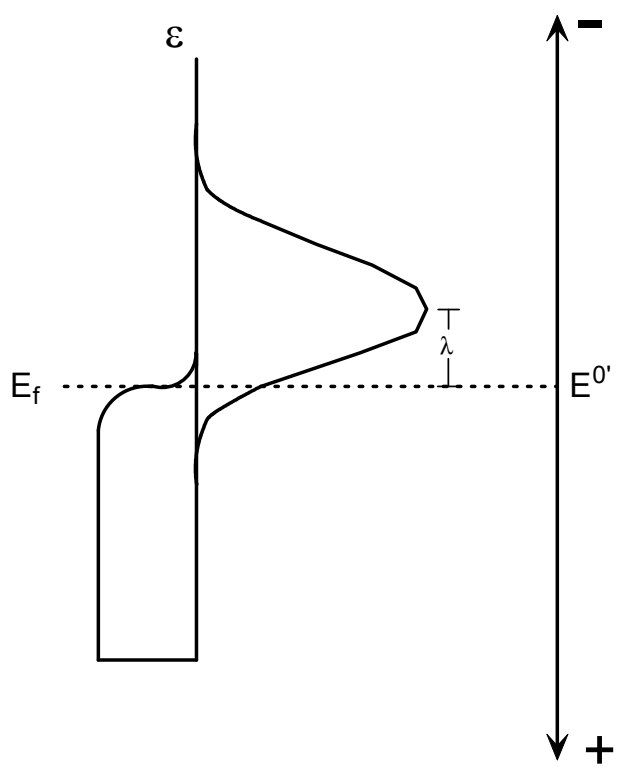

b.

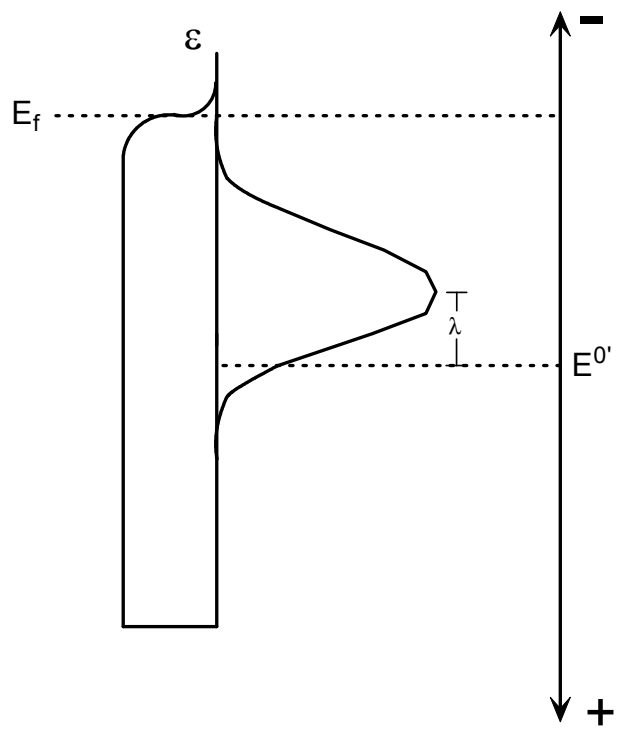

C.

Figure 1.7.1 Marcus Density of States Diagrams at various overpotentials; $E \gg E_{0}^{\prime}$ for (a), $E=E_{0}^{\prime}$ for (b), $E<<E_{0}^{\prime}$ for (c). * Density of metallic electronic states ${ }^{* *}$ Density of redox electronic states 
(donor) of the metal and the empty states (acceptor) of the analyte. Thus, reduction current is very small. In Figure 1.7.1.b, the potential is held negative of the formal potential. Overlap is significant, and reduction current (and consequently the rate) increases. In Figure 1.7.1.c, the Fermi level is at a highly negative potential, and there is considerable overlap with acceptor states. The reductive current is much higher, and reaches a maximum when there is complete overlap between the metal donor states and the redox couples acceptor states. The current/overpotential relationship is represented in Figure 1.7.2. Note the limiting rate as overlap becomes complete. In homogenous electron transfer reactions, Marcus theory predicts that the rate of the reaction first increases, then decreases as the driving force, $\Delta G^{0}=n F E^{0}$, increases. The latter region is called the "inverted" region. The "inverted" region occurs because overlap of the two Gaussian distributions representing the density of complementary states will increase as the two distributions approach similar energies, reaches a maximum where the two distributions are of the same energy, then declines as the energy difference between the two distributions increase. When using an electrode as the second "reactant", there is no "inverted" region as predicted by general Marcus theory because all donor states on the electrode are occupied at all potentials below (more positive than) the Fermi energy. Thus as the Fermi energy increases above the energy of the acceptor states, saturation occurs, and the curve reaches a limiting value.

Marcus Theory also describes the effect of the reorganization energy on standard rate constant using the following relation, where the overpotential is small with respect to the reorganization energy ${ }^{5}$ 


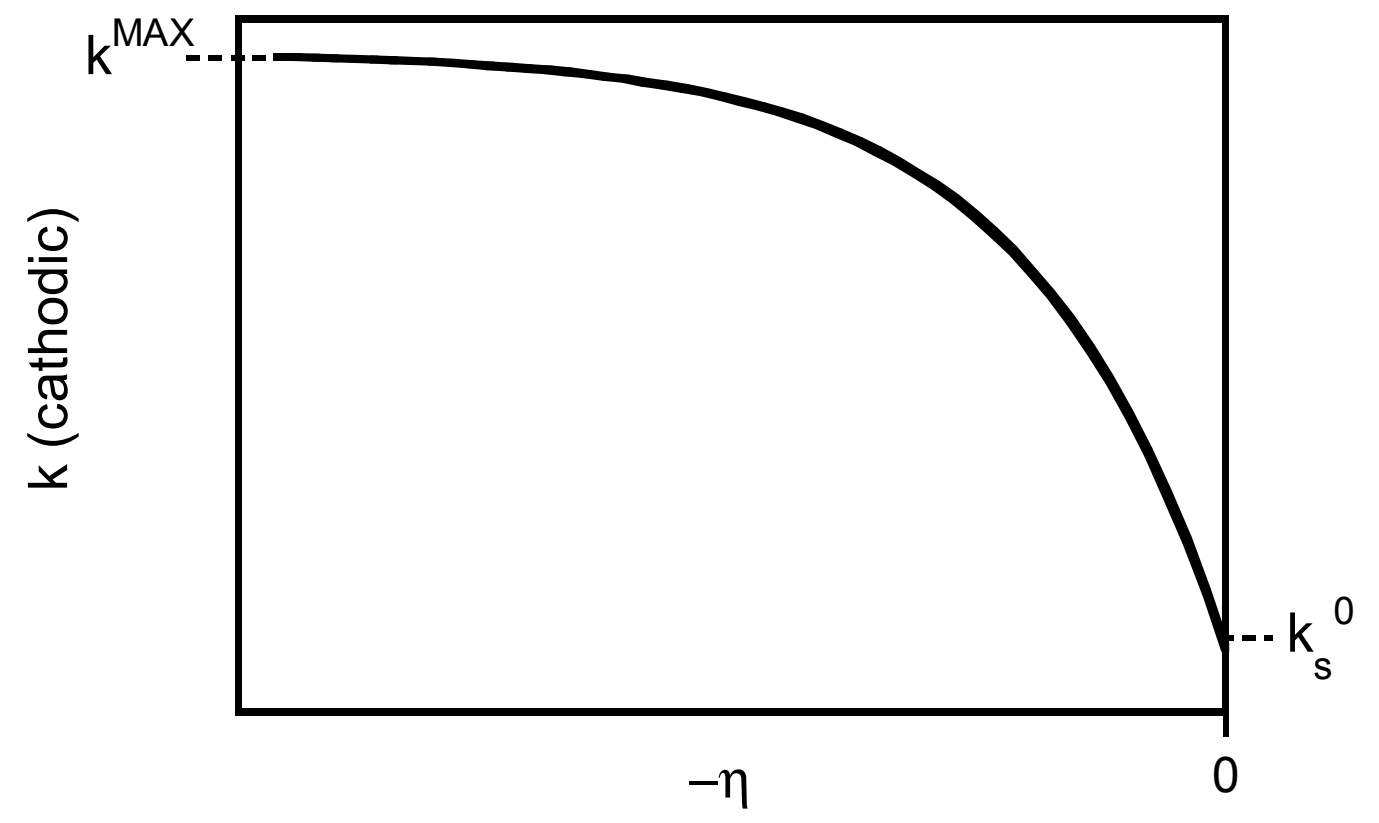

Figure 1.7.2 Dependence of reductive rate on negative overpotential. Note the limiting rate at high negative overpotentials 


$$
\mathrm{k}_{\mathrm{s}} \approx \mathrm{e}^{\frac{-\lambda}{4 \mathrm{kT} T}}
$$

Because of this relationship, when all other factors are similar, an increase in $\lambda$ results in a decrease in $\mathrm{k}_{\mathrm{s}}$, and vice versa.

\subsection{Marcus Theory Predictions}

The numerical evaluation of rate equation (1.7.5) can be displayed using Tafel analysis (In k vs $\eta$ ). The most notable feature of the Marcus DOS model is that it predicts curvature in the Tafel plots. Figure 1.8.1 is a theoretical overlay plot of $\log (\mathrm{k})$ vs $\eta$ for different reorganization energies. Note the curvature in the plot, which is a distinct departure from the expected shape as predicted by the Butler-Volmer approach. Tafel plots using the Butler-Volmer approach were linear until they reached mass transport limiting rates. Marcus theory predicts that there is a linear section at low overpotential ( $|\eta|<e \lambda)$, which matches the Butler-Volmer theory. However at higher overpotentials, curvature due to the magnitude of the reorganization energy of the process becomes significant. Because the curvature of the plots is based on reorganization energy alone, it is a simple process to extract the magnitude of the reorganization energy based on the extent of curvature of the plot. This has also been reported extensively ${ }^{6 ; 11 ; 12}$. Thus it is possible to extract both the standard rate constant and the reorganization energy from a Tafel plot covering a sufficient range of overpotentials. 


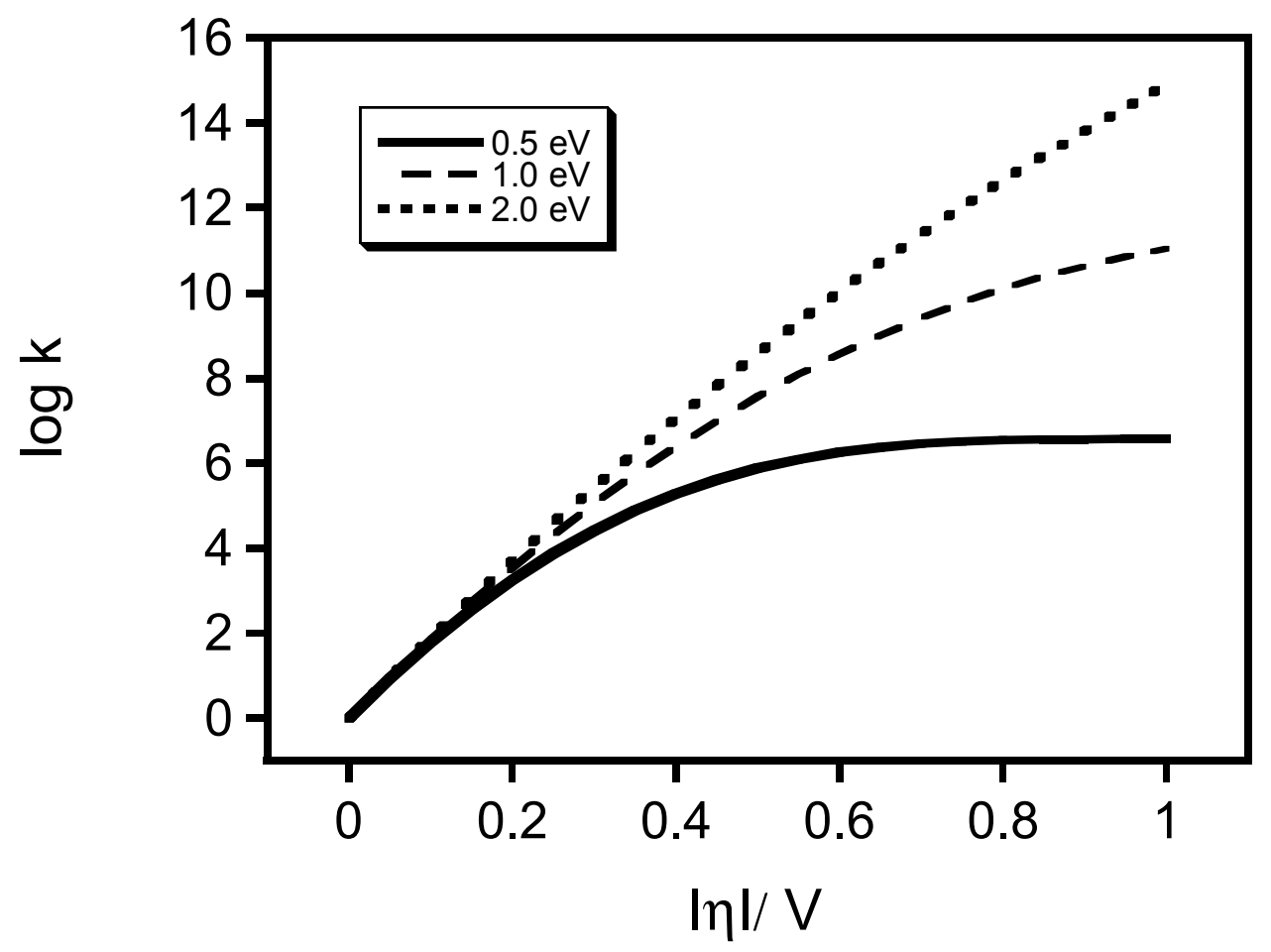

Figure 1.8. 1 Dependence of Curvature of Tafel Plot on Reorganization Energy ${ }^{8}$ 
The Butler-Volmer model shows that the slope of a Tafel plot is proportional to the transfer coefficient. Because the transfer coefficient stays constant at all overpotentials, the slope of the plot is constant as well. Curvature of Tafel plots implies that the transfer coefficient is potential dependent. This concept is in direct conflict with the Butler-Volmer model. Eq (1.7.5) yields Tafel plots from eqs (1.1.21) and (1.1.22), with the transfer coefficient as defined by each branch as

$$
\begin{gathered}
\alpha=-\frac{2.3 \mathrm{RT}}{\mathrm{F} \eta} \log \left(\frac{\mathrm{k}_{\mathrm{c}}}{\mathrm{k}_{\mathrm{s}}{ }^{0}}\right) \\
\alpha=1-\frac{2.3 \mathrm{RT}}{\mathrm{F} \eta} \log \left(\frac{\mathrm{k}_{\mathrm{a}}}{\mathrm{k}_{\mathrm{s}}^{0}}\right)
\end{gathered}
$$

As the overpotential becomes increasingly positive, alpha increases. This can be seen in Figure 1.8.2, which shows the effect that the reorganization energy has on the potential dependence of the transfer coefficient. The potential dependence of the transfer coefficient on overpotential has been proven by fitting experimental Tafel plots with theoretical Tafel plots as calculated by equation (1.7.5), with $\lambda$ as the fitting parameter. It has been shown by Finklea ${ }^{8}$ that the potential dependence of the transfer coefficient on potential can be accurately simulated by the following polynomial equation

$$
\alpha(\eta)=0.5+a \eta+b \eta^{3}+c \eta^{5}
$$

where $a, b$,and $c$ are coefficients dependent solely on the reorganization energy of the system. 


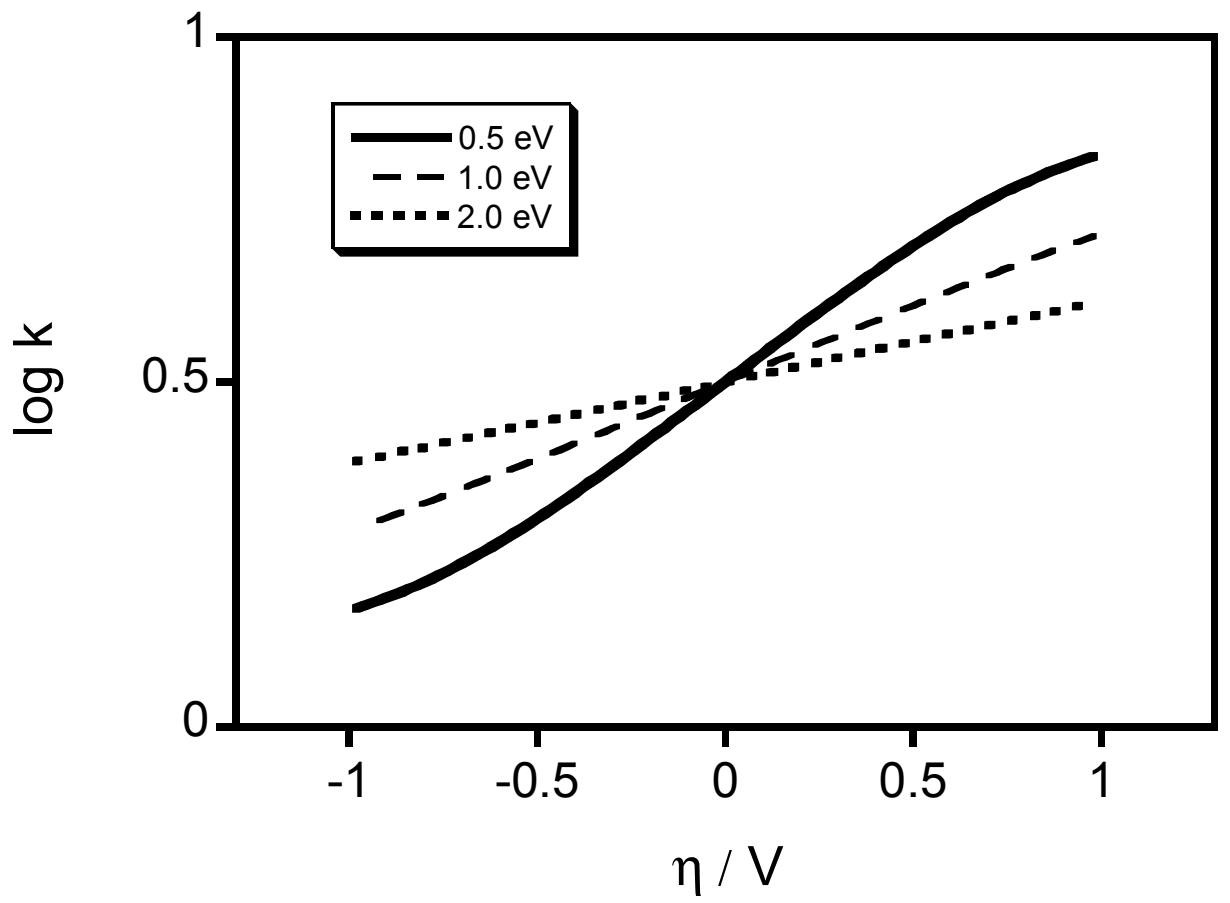

Figure 1.8. 2 Dependence of Transfer Coefficeint on Reorganization Energy $^{8}$ 
The symmetry of the Tafel plot also can yield information about the system. Tafel plot symmetry is a result of having equal reorganization energies for both the anodic and cathodic branches. Since the outer sphere contribution is independent of charge, a complex with an outer sphere dominated reorganization energy should have equal $\lambda$ 's for both branches. However, when inner sphere contributions are significant, the reorganization energy for oxidation might differ from that of reduction. In these cases, it is expected that the Tafel plots show asymmetry, with one branch curving at a faster rate than the other.

\subsection{Introduction to insulating electrodes}

As discussed earlier, there are a number of inherent difficulties with probing the kinetics of a redox complex at the surface of an electrode in free solution. The rate of electron transfer for the species is typically high. Such a system requires specialized equipment. Also, the fast potential transients required to extract kinetic information result in significant $\mathrm{iR}$ drop (which will be discussed later). More importantly, when

standard rate constants, $\mathrm{k}_{\mathrm{s}}{ }^{0}$, are above $0.1 \mathrm{~cm} / \mathrm{s}$, it is hard to measure $\mathrm{k}_{\mathrm{s}}{ }^{0}$ or $\mathrm{k}$ because mass transfer tends to be rate-limiting. This can lead to difficulties arising because mass transport limiting currents are reached at overpotentials not sufficient for extracting kinetic information. Double layer effects are prominent, as discussed in Section 1.7, and can lead to kinetic and thermodynamic heterogeneity. Because of the 
many sources for error, kinetic measurements in free solution with a bare electrode can be experimentally difficult.

The introduction of insulating electrodes has improved the ease with which kinetic information can be extracted from a redox couple. By coating the working electrode with a well defined thin layer of an insulating material, and attaching the redox species to this monolayer, the closest proximity of the redox complex to the electrode surface is limited, which reduces the electronic coupling between the complex and the electrode. The rate of electron transfer is proportional to the electronic coupling contribution, thus, the rate of electron transfer is lowered. The rate of electron transfer can be lowered systematically by increasing the insulator thickness by a given amount. This feature is very important in that it allows the analysis of fast systems by lowering the rate of electron transfer into a regime that can be measured by conventional means.

The most prominent form of electrode insulation used in the attachment of redox couples in recent years has been self-assembled thiol monolayers. Self-assembled monolayers (SAM's) are widely used because they form a well organized, tightly packed insulating surface ${ }^{11}$. The thiol head group adsorbs strongly to a number of different electrode materials (Au and Pt, are typical). The ability of SAM's to form uniform, tightly packed layers is essential to providing a proper medium for insulation. Ideally an insulating film should reduce the electronic coupling between the electrode and the redox molecule by a consistent amount regardless of the spatial position of the molecule around the electrode. A non-uniform film would introduce its own double 
layer effect because some redox molecules would be closer to the electrode than others. One of the most interesting benefits of using a SAM is that it is possible to systematically control the rate of electron transfer by simply increasing the length of the alkyl chain of the monolayer. It has been shown by C. J. Miller ${ }^{7}$ that the strength of electronic coupling decays exponentially with the number of methylene units within the monolayer according to the following equation

$$
\mathrm{k}=\mathrm{k}^{0} \mathrm{e}^{-\beta n}
$$

where $\mathrm{k}^{0}$ is the rate constant with no $\mathrm{CH}_{2}$ 's present, $\beta$ is the tunneling parameter and $\mathrm{n}$ is the number of methylene units within the monolayer. Miller has also shown that the tunneling parameter is dependent on the nature of the monolayer, but is relatively independent of the nature of the redox complex or electrode potential ${ }^{7}$. This is highly desirable because it suggests that the monolayer will reduce the rate of electron transfer by a predictable factor.

\subsection{Redox centers attached to self-assembled monolayers}

Further experimental improvements can be achieved by attaching the electroactive species to the monolayer instead of having it contained in the bulk solution. Figure 1.10.1 is representative of a long chain thiol with an attached redox couple, adsorbed to a gold electrode. Attaching a redox molecule serves two basic purposes: it fixes the concentration of the analyte to the number of molecules attached to the SAM and it fixes the distance of the redox molecule from the electrode. 
Self-assembled Monolayer with attached Redox Centers

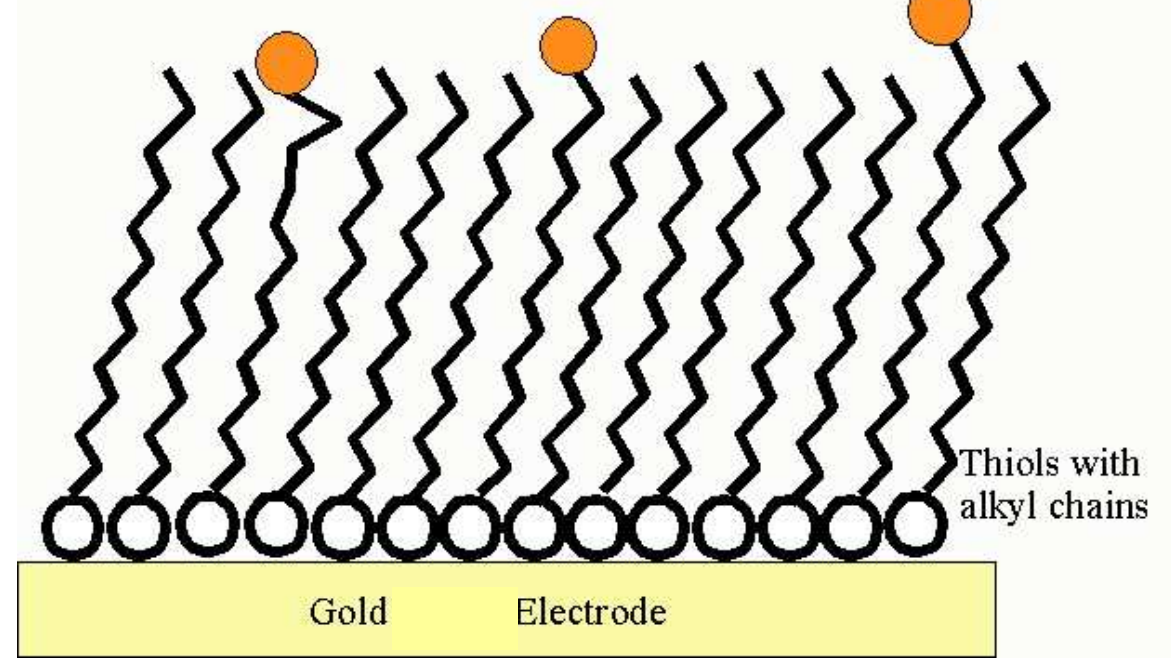

Figure 1.10. 1 adsorbed to an electrode ${ }^{13}$

Diagram of monolayer with attached redox centers 
Attaching the redox complex to the monolayer effectively eliminates all mass transfer. This simplifies the theory in that the concentration at a specific time is always equal to the "bulk" concentration, which is simply the coverage of the redox center on the electrode. The largest consequence is that there is no longer an overpotential range that will be mass transfer limiting.

For attached redox centers, the equilavent rate expressions are

$$
\begin{aligned}
& \text { Oxidation rate }=\mathrm{k}_{\mathrm{a}} \Gamma_{\mathrm{R}}(\mathrm{t}) \\
& \text { Reduction rate }=\mathrm{k}_{\mathrm{c}} \Gamma_{\mathrm{o}}(\mathrm{t})
\end{aligned}
$$

where $\Gamma_{R}(t)$ and $\Gamma_{0}(t)$ are surface concentrations of each attached species in $\mathrm{mol} / \mathrm{cm}^{2}$. Since oxidation/reduction rate is defined in units of $\mathrm{mol} /\left(\mathrm{cm}^{2}-\mathrm{s}\right)$, units of $\mathrm{k}_{\mathrm{c}}$ and $\mathrm{k}_{\mathrm{a}}$ are $\mathrm{s}^{-1}$. There is no flux of analyte to the electrode surface, thus there are no mass transfer limitations. Another benefit of using a redox couple attached to an insulator lies in the fact that double layer effects are greatly reduced, because all redox centers should be fixed at a specific distance from the electrode. In an ideal situation, double layer effects should be minimized because of the increased distance from the electrode and the presence of a low dielectric constant layer between the electrode and the redox center. In practice, double layer effects can still be problematic depending on the nature of the monolayer. Attaching a redox couple to the end of the monolayer can cause packing difficulties which can lead to the burial of some redox centers into the monolayer, which 
can cause a distribution of formal potentials and electron transfer rates. Details on monolayer structure and minimization of error will be provided in a later section.

\subsection{Introduction to Proton Coupled Electron Transfer Reactions}

Discussion so far has been centered around simple electron transfer reactions. An important class of electron transfer reactions involves both proton and electron transfer. Proton Coupled Electron Transfer (PCET) reactions play a vital role in a variety of biological and chemical processes. Examples of PCET systems include redox-driven proton pumps in mitochondria ${ }^{14}$, photosynthesis ${ }^{15-17}$, neurotransmission ${ }^{18 ; 19}$, and enzymatic reactions ${ }^{20}$. Relevant biological molecules exhibiting PCET include quinones and catechols, flavins, and NAD/NADH. PCET reactions are also relevant in the areas of metal corrosion and radioactive waste disposal ${ }^{14}$. As a first step in understanding the intricacies of biological PCET reactions, it is necessary to explore PCET reactions at a fundamental level. A comprehensive theory was developed by E. Laviron in the 1980's to explain the behavior of simple proton/electron transfer systems ${ }^{21-29}$. In this project, Finklea has re-derived some of the kinetic theory from Laviron's original work ${ }^{8 ; 30}$. Finklea's theory mirrors Laviron's theory in content; however some aspects have been reworded in order to simplify terminology as well as incorporate aspects of Marcus DOS Theory. Implications of Finklea's derivation will be discussed later. 
The general reaction scheme for a 1-electron, 1-proton $(1 \mathrm{e} 1 \mathrm{H})$ transfer reaction is as follows

$$
\mathrm{O}+\mathrm{e}^{-}+\mathrm{H}^{+} \rightleftharpoons \mathrm{R}
$$

where $\mathrm{O}$ is the oxidized/deprotonated form and $\mathrm{R}$ is the reduced/protonated form. In her derivation, Laviron made a few general assumptions ${ }^{23}$. The first assumption is that electron transfer and proton transfer are two distinct, separate steps. With this assumption, the reaction can be described by the square-scheme described in Figure 1.11.1, where $P$ is the reduced/deprotonated form and $Q$ is the oxidized/protonated form. Note that reduction from $\mathrm{O}$ to $\mathrm{R}$ can occur via two separate paths. O-P-R is the electron transfer then proton transfer path $(\mathrm{eH})$ and $\mathrm{O}-\mathrm{Q}-\mathrm{R}$ is the proton transfer then electron transfer path $(\mathrm{He})$. Thermodynamic and kinetic parameters are defined for each step in the reaction: $E_{2}{ }^{0}, k_{s 2}, \alpha_{2}$ are involved in O-P electron transfer step, $E_{1}{ }^{0}$, $\mathrm{k}_{\mathrm{s} 1}, \alpha_{1}$ are involved in the $\mathrm{Q}-\mathrm{R}$ electron transfer step, and $\mathrm{K}_{\mathrm{a} 2}$ and $\mathrm{K}_{\mathrm{a} 1}$ are the acid disassociation constants associated with the P-R and O-Q proton transfer steps.

Another assumption made by Laviron is that proton transfer is at equilibrium; the rate of proton transfer is much faster than the rate of electron transfer. With these assumptions, the system behaves as a simple 1-electron reaction with the $\mathrm{pH}$ dependent apparent formal potential $E_{r}$ :

$$
\mathrm{E}_{\mathrm{r}}=\mathrm{E}_{2}{ }^{0}+\frac{2.3 \mathrm{RT}}{\mathrm{F}} \log \left[\frac{1+\frac{\left[\mathrm{H}^{+}\right]}{\mathrm{K}_{\mathrm{a} 2}}}{1+\frac{\left[\mathrm{H}^{+}\right]}{\mathrm{K}_{\mathrm{a} 1}}}\right]
$$




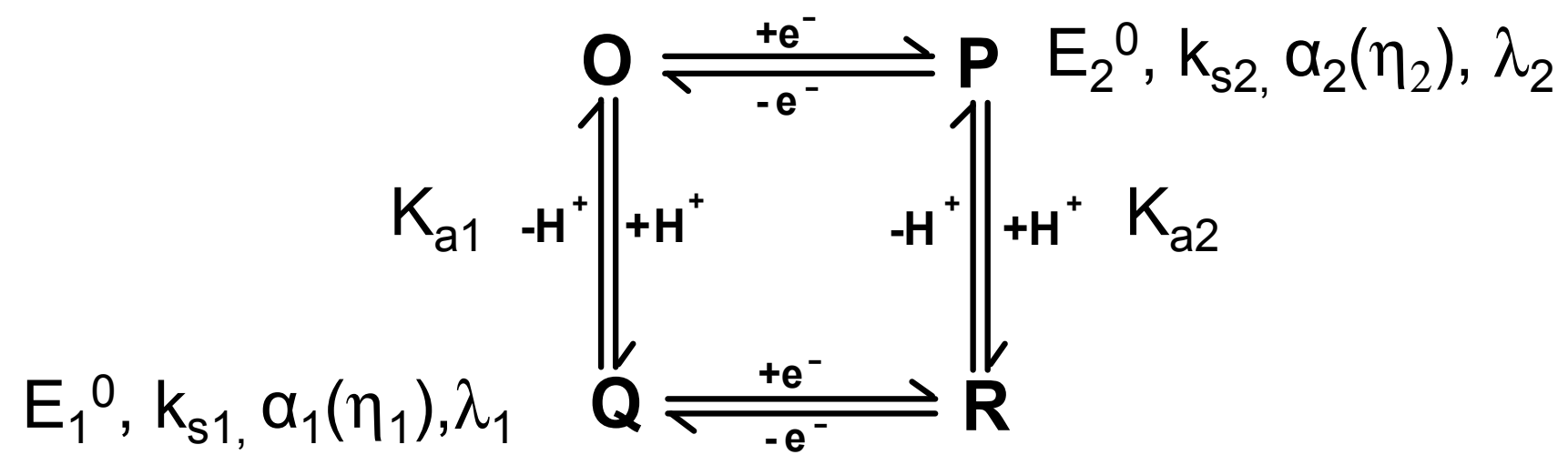

Figure 1.11. 1

1e1H Square Scheme 
The potential vs. $\mathrm{pH}$ profile generated by equation (1.11.2) is seen in Figure 1.11.2. In order to simplify future discussion, low $\mathrm{pH}$ is going to be defined as any $\mathrm{pH}$ where $\mathrm{pH}<\mathrm{pK}_{\mathrm{a} 1}$, intermediate $\mathrm{pH}$ is where $\mathrm{pK}_{\mathrm{a} 1}<\mathrm{pH}<\mathrm{pK}_{\mathrm{a} 2}$, and high $\mathrm{pH}$ is where $\mathrm{pK}_{\mathrm{a} 2}<\mathrm{pH}$. As shown in the graph, at low $\mathrm{pH}, \mathrm{E}_{\mathrm{r}}=\mathrm{E}_{1}{ }^{0}$. At intermediate $\mathrm{pH}, \mathrm{E}_{\mathrm{r}}$ shifts $-59 \mathrm{mV} / \mathrm{pH}$, and at high $\mathrm{pH}, \mathrm{E}_{\mathrm{r}}=\mathrm{E}_{2}^{0}$. The separation between both formal potentials is defined as:

$$
\mathrm{E}_{1}^{0^{\prime}}-\mathrm{E}_{2}^{0^{\prime}}=(2.3 \mathrm{RT} / \mathrm{F})\left(\mathrm{pK}_{\mathrm{a} 2}-\mathrm{pK}_{\mathrm{a} 1}\right)
$$

Because of the two pathways shown in Figure 1.11.1, overpotential must now be defined with respect to each pathway as follows:

$$
\eta_{1}=\mathrm{E}-\mathrm{E}_{1}^{0^{\prime}}
$$

for the Q-R half reaction, and

$$
\eta_{2}=\mathrm{E}-\mathrm{E}_{2}^{0^{\prime}}
$$

for the O-P half reaction. The overall rate can be described as a summation of the rates of both paths using the general form derived from eq (1.1.19)

$$
\begin{array}{r}
\text { Apparent Rate }=\mathrm{k}_{\mathrm{s} 1}\left[\Gamma_{\mathrm{R}} \mathrm{e}^{\left(1-\alpha_{1}\right) f \eta_{1}}-\Gamma_{\mathrm{Q}} \mathrm{e}^{-\alpha_{1} \mathrm{f \eta _{1 }}}\right]+ \\
\mathrm{k}_{\mathrm{s} 2}\left[\Gamma_{\mathrm{P}} \mathrm{e}^{\left(1-\alpha_{2}\right) f \eta_{2}}-\Gamma_{\mathrm{O}} \mathrm{e}^{-\alpha_{2} \mathrm{f} \eta_{2}}\right]
\end{array}
$$

where $\Gamma_{x}$ is the surface concentration of each species. Because protonation is at equilibrium

$$
\mathrm{K}_{\mathrm{a} 1}=\frac{\left[\mathrm{H}^{+}\right] \Gamma_{\mathrm{O}}}{\Gamma_{\mathrm{Q}}} \text { and } \mathrm{K}_{\mathrm{a} 2}=\frac{\left[\mathrm{H}^{+}\right] \Gamma_{\mathrm{P}}}{\Gamma_{\mathrm{R}}}
$$




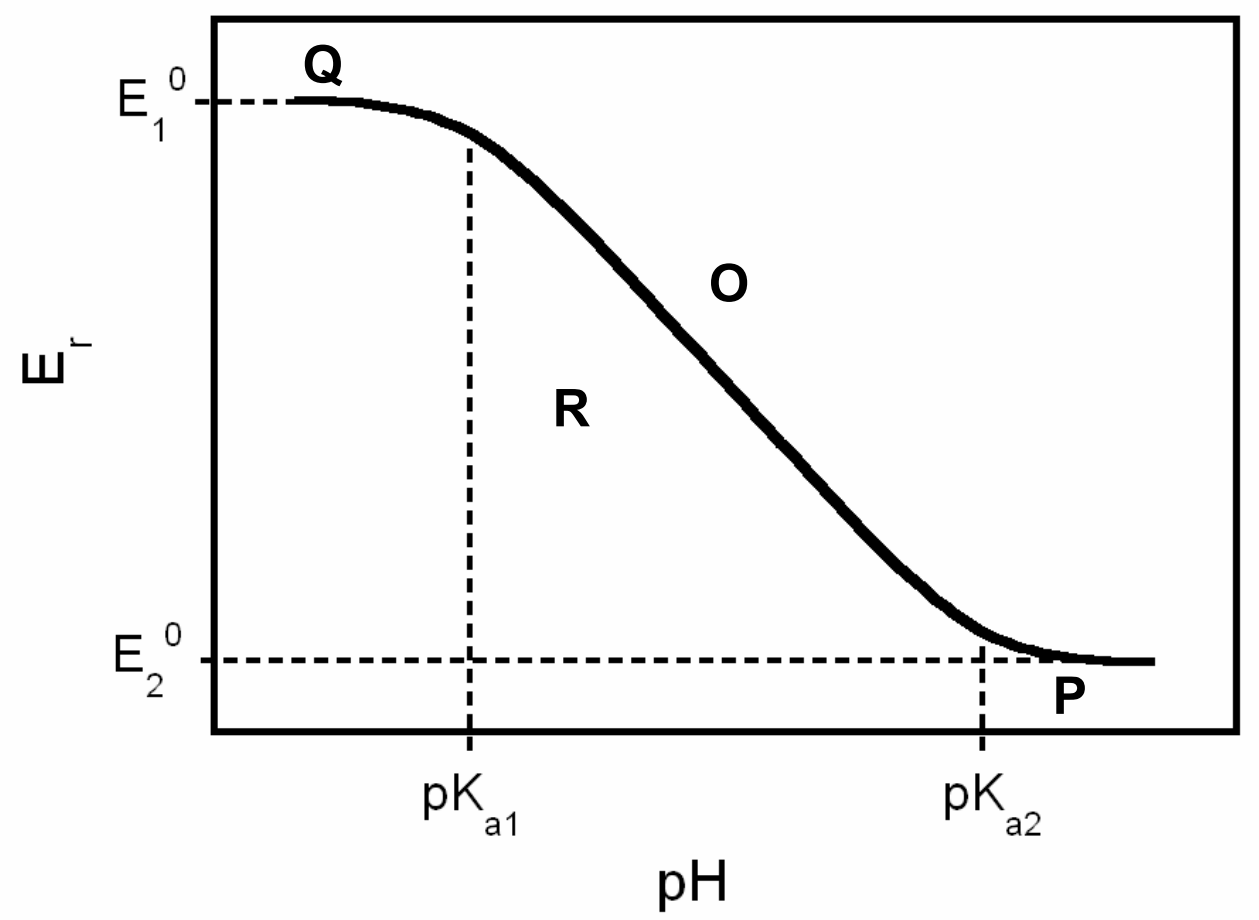

Figure 1.11. 2

Theoretical $\mathrm{E}_{\mathrm{r}} \mathbf{v s} \mathrm{pH}^{31}$ 
Substitution for $\Gamma_{\mathrm{Q}}$ and $\Gamma_{\mathrm{P}}$ yields the following expression

$$
\begin{aligned}
\text { Apparent Rate }\left(\mathrm{k}_{\mathrm{app}}\right)=\mathrm{k}_{\mathrm{s} 1}[ & \left.\Gamma_{\mathrm{R}} \mathrm{e}^{\left(1-\alpha_{1}\right) \mathrm{f}_{1}}-\left(\frac{\left[\mathrm{H}^{+}\right] \Gamma_{\mathrm{O}}}{\mathrm{K}_{\mathrm{a} 1}}\right) \mathrm{e}^{-\alpha_{1} \mathrm{fn}_{1}}\right] \\
& +\mathrm{k}_{\mathrm{s} 2}\left[\left(\frac{\mathrm{K}_{\mathrm{a} 2} \Gamma_{\mathrm{R}}}{\left[\mathrm{H}^{+}\right]}\right) \mathrm{e}^{\left(1-\alpha_{2}\right) \mathrm{f \eta}}-\Gamma_{\mathrm{O}} \mathrm{e}^{-\alpha_{2} \mathrm{f \eta}_{2}}\right]
\end{aligned}
$$

This overall expression for rate can be separated into its cathodic and anodic components and placed over the respective total surface concentration of reduced $\left(\Gamma_{\text {red }}=\Gamma_{\mathrm{Q}}+\Gamma_{\mathrm{R}}\right)$ and oxidized $\left(\Gamma_{\mathrm{ox}}=\Gamma_{\mathrm{O}}+\Gamma_{\mathrm{P}}\right)$ species to yield the expressions for the cathodic and anodic rate constants

$$
\begin{aligned}
& \mathrm{k}_{\mathrm{c}, \mathrm{app}}=\frac{\left(\frac{\left[\mathrm{H}^{+}\right]}{\mathrm{K}_{\mathrm{a} 1}}\right) \mathrm{k}_{\mathrm{s} 1} \mathrm{e}^{-\alpha_{1} \mathrm{f} \eta_{1}} \mathrm{k}_{\mathrm{s} 2} \mathrm{e}^{-\alpha_{2} \mathrm{f \eta}} 2}{1+\frac{\left[\mathrm{H}^{+}\right]}{\mathrm{K}_{\mathrm{a} 1}}} \\
& \mathrm{k}_{\mathrm{a}, \mathrm{app}}=\frac{\mathrm{k}_{\mathrm{s} 1} \mathrm{e}^{\left(1-\alpha_{1}\right) \mathrm{f} \eta_{1}}+\left(\frac{\mathrm{K}_{\mathrm{a} 2}}{\left[\mathrm{H}^{+}\right]}\right] \mathrm{k}_{\mathrm{s} 2} \mathrm{e}^{\left(1-\alpha_{2}\right) \mathrm{f} \eta_{2}}}{1+\frac{\mathrm{K}_{\mathrm{a} 2}}{\left[\mathrm{H}^{+}\right]}}
\end{aligned}
$$

The apparent standard rate constant, $k_{s, a p p}$ can be calculated by setting $E=E_{r}$. Thus overpotentials are defined with respect to the reversible potential. At $E=E_{r}$, 


$$
\mathrm{k}_{\mathrm{s}, \mathrm{app}}=\mathrm{k}_{\mathrm{c}, \mathrm{app}}=\mathrm{k}_{\mathrm{a}, \mathrm{app}}
$$

The $1 \mathrm{p} 1 \mathrm{e}$ square scheme shows that the reaction can progress through either pathway (eH or $\mathrm{He})$. From eq (1.11.9), the percent of the eH path is defined as the eH contribution divided by the entire contribution

$$
\% \operatorname{path}(\mathrm{eH})=\frac{\left(\left[\frac{\left[\mathrm{H}^{+}\right]}{\mathrm{K}_{\mathrm{a} 1}}\right) \mathrm{k}_{\mathrm{s} 1} \mathrm{e}^{-\alpha_{1} \mathrm{f \eta}} 1\right.}{\left(\frac{\left[\mathrm{H}^{+}\right]}{\mathrm{K}_{\mathrm{a} 1}}\right) \mathrm{k}_{\mathrm{s} 1} \mathrm{e}^{-\alpha_{1} \mathrm{f \eta}} 1+\mathrm{k}_{\mathrm{s} 2} \mathrm{e}^{-\alpha_{2} \mathrm{f \eta}} 2} \times 100
$$

\%path $(\mathrm{He})=1-\%$ path $(\mathrm{eH})$, and can also be calculated from eq (1.11.10).

The differences between Laviron's and Finklea's assumptions affect only the kinetics of electron transfer. Qualitatively, both theories yield similar results, but quantitatively they differ. The consequences of each case will be discussed separately.

\subsection{Kinetic Predictions using Laviron Theory}

Laviron's two main assumptions are (1) proton transfer is much faster than electron transfer, and thus it doesn't contribute to the overall rate and (2) the transfer coefficient of electron transfer is 0.5 at all overpotentials. The second assumption results in a number of interesting consequences. The most noticeable consequence is that voltammogram shapes remain symmetrical at all scan rates and over all pH's. 
This symmetry is also seen in Tafel plots, where there is mirror symmetry between the anodic and cathodic branches. Another interesting feature of the Tafel plots is that they are linear as described by Butler-Volmer theory, as detailed in Section 1.2. Figure 1.2.1 is an example of this kind of Tafel plot.

Quantitatively, consequences of Laviron's derivation are seen in the apparent standard rate constant. By looking at eqs (1.11.9) to (1.11.11) it can be seen that $\mathrm{k}_{\mathrm{s}, \mathrm{app}}$ is dramatically dependent on $\mathrm{pH}$. Figure 1.12 .1 shows the relationship between $\mathrm{k}_{\mathrm{s}, \mathrm{app}}$ and $\mathrm{pH}$. Note that this is a generic diagram and it is assumed that $\mathrm{k}_{\mathrm{s} 1}=\mathrm{k}_{\mathrm{s} 2}$. At low and high $\mathrm{pH}, \mathrm{k}_{\mathrm{s}, \mathrm{app}}=\mathrm{k}_{\mathrm{s} 1}=\mathrm{k}_{\mathrm{s} 2}$. However, at intermediate $\mathrm{pH}$ there is an extreme dependence of the apparent rate on $\mathrm{pH}$. The rate decreases approximately an order of magnitude per $2 \mathrm{pH}$ units until $\mathrm{pK}_{\text {mid }}$, which is is defined as the $\mathrm{pH}$ halfway between $\mathrm{pK}_{\mathrm{a} 1}$ and $\mathrm{pK}_{\mathrm{a} 2}$, is reached. There is a minimum at $\mathrm{pK}_{\text {mid. }}$. From eq (1.11.12), it can also be shown that the path of electron transfer is independent of overpotential and dependent only on $\mathrm{pH}$.

A significant concern in examining PCET reactions using the Laviron approximation is the assumption that the transfer coefficient is 0.5 at all overpotentials. Just as it has been shown that the assumption made using the Butler-Volmer derivation ( $\alpha$ is independent of the potential) is not sufficient to describe simple electron transfer, this deficiency is likely to apply to proton-coupled electron transfer systems. The next section discusses the consequences of a potential-dependent transfer coefficient on the thermodynamics and kinetics. 


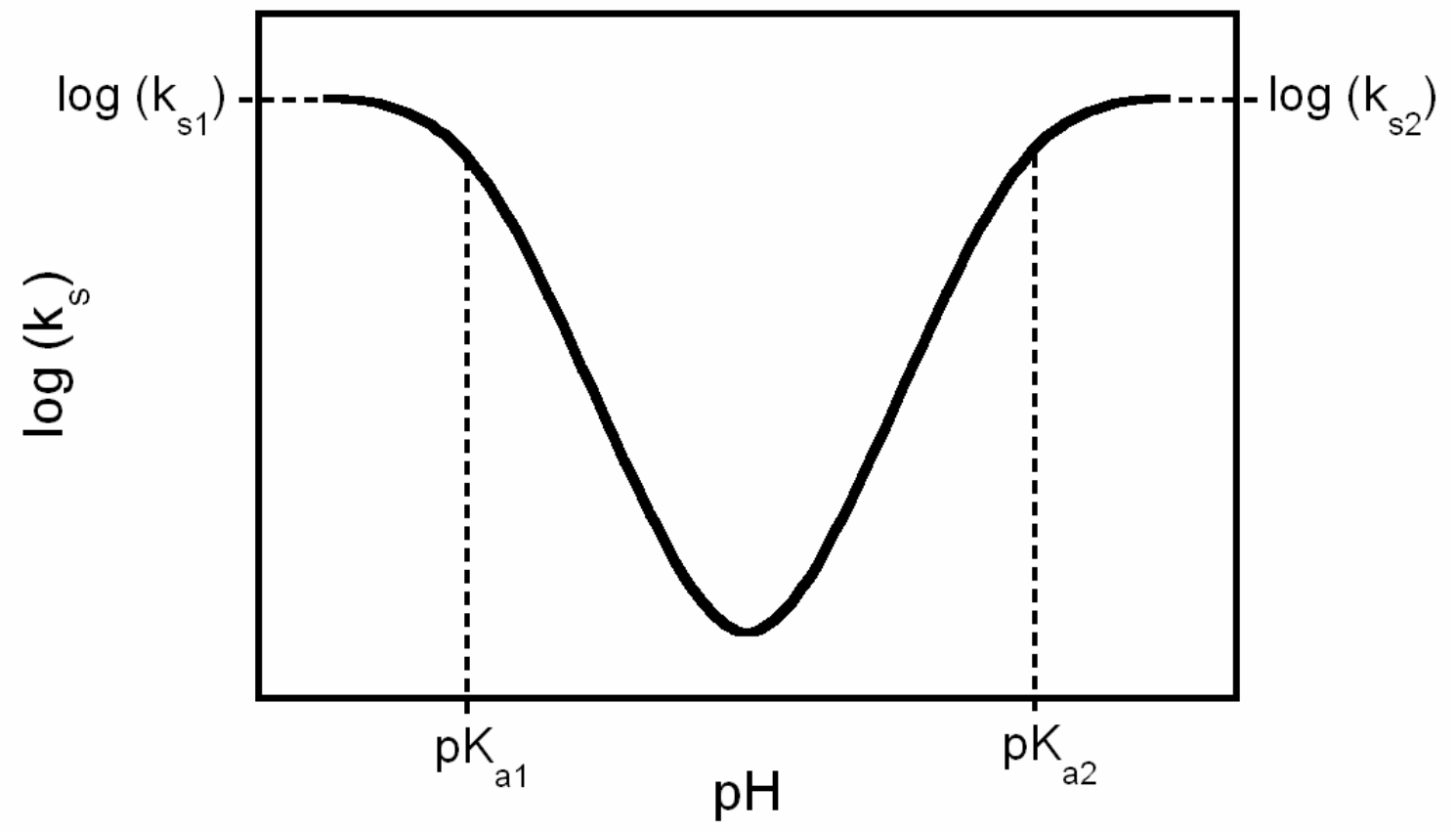

Figure 1.12. 1 Theoretical $\log \mathbf{k}_{\mathbf{s}}$ vs $\mathbf{p H}$ using Laviron analysis ${ }^{31}$ 


\subsection{Kinetic Predictions Using Finklea Theory}

The Finklea model of PCET applies Marcus DOS theory to Laviron's model. The Finklea model allows for a potential-dependent transfer coefficient, whereas Laviron's model requires that the transfer coefficient be 0.5 at all overpotentials. The thermodynamics remain unchanged, as derived in Section 1.11, but adding a potentialdependent $\alpha$ causes significant changes in the kinetics of a PCET system. The first consequence is that voltammograms contain curvature as described by Marcus DOS theory from Section 1.8. A related consequence involves the apparent transfer coefficient at zero overpotential, $\alpha(0) . \alpha_{a p p}$ is defined with a modification eqs (1.11.4) and (1.11.5) as follows:

$$
\alpha_{\text {c,app }}=-\frac{\mathrm{RT}}{\mathrm{F} \eta} \log \left(\frac{\mathrm{k}_{\mathrm{c}}(\eta)}{\mathrm{k}_{\mathrm{s}, \mathrm{app}}}\right)
$$

or

$$
\alpha_{\mathrm{a}, \mathrm{app}}=1-\frac{\mathrm{RT}}{\mathrm{F} \eta} \log \left(\frac{\mathrm{k}_{\mathrm{a}}(\eta)}{\mathrm{k}_{\mathrm{s}, \mathrm{app}}}\right)
$$

Extrapolation of these potential-dependent $\alpha$ 's to zero overpotential yields $\alpha(0)$. The theoretical potential dependence of $\alpha(0)$ vs $\mathrm{pH}$ is shown in Figure 1.13.1. $\alpha(0)$ equals

0.5 at high $\mathrm{pH}$, low $\mathrm{pH}$, and exactly at $\mathrm{pK}_{\text {mid. }}$. At $\mathrm{pK}_{\mathrm{a} 1}<\mathrm{pH}<\mathrm{pK}_{\text {mid }}, \alpha(0)<0.5$.

Conversely, at $\mathrm{pK}_{\text {mid }}<\mathrm{pH}<\mathrm{pK}_{\mathrm{a} 2}, \alpha(0)>0.5$. The two main consequences of this feature are that voltammograms and Tafel plots are no longer symmetrical. pH's close 


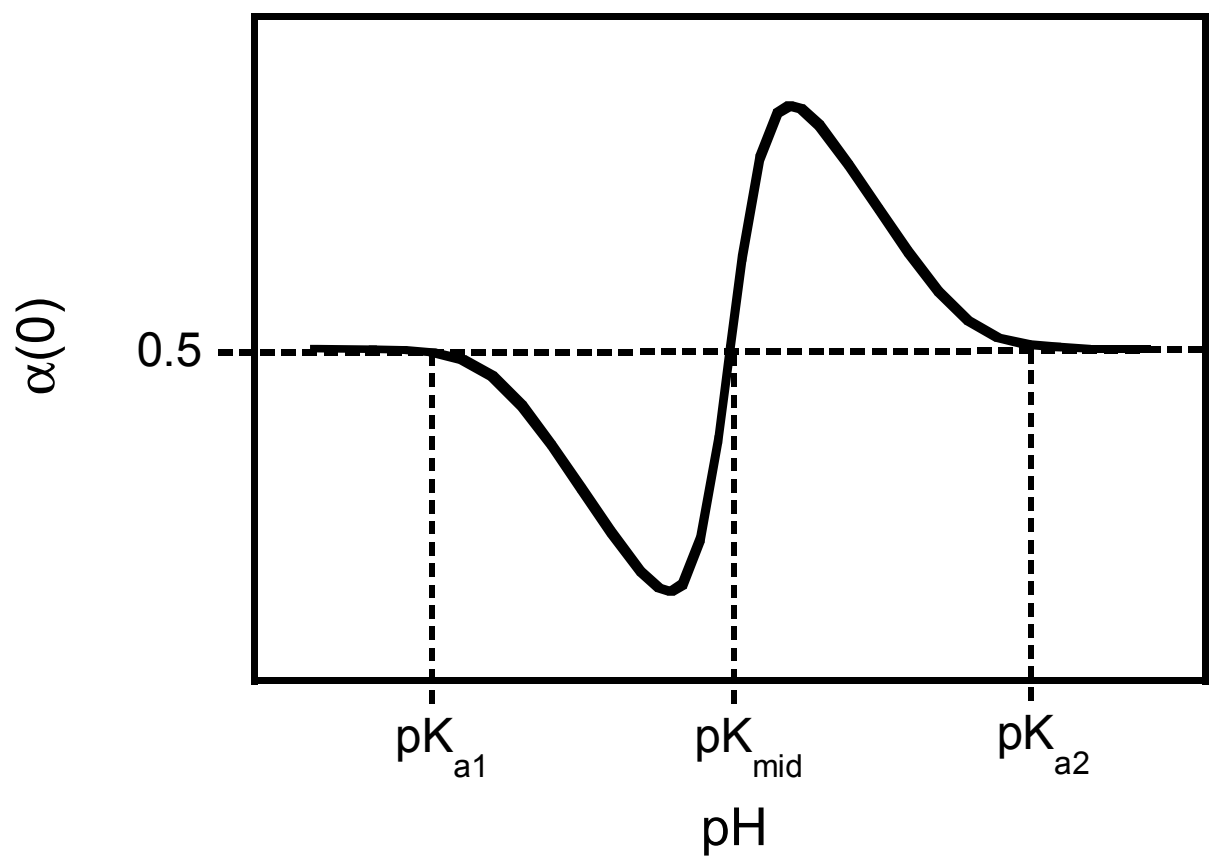

Figure 1.13. 1 Theoretical $\alpha(0)$ vs $\mathrm{pH}^{31}$ 
to $\mathrm{pK}_{\mathrm{a} 2}$ will result in voltammograms where the anodic peak is broader than the cathodic peak, and the reverse will apply at $\mathrm{pH}$ 's close to $\mathrm{pK}_{\mathrm{a} 1}$. This asymmetry is carried over into Tafel plots, where at $\mathrm{pH}$ 's close to $\mathrm{pK}_{\mathrm{a} 2}$, the anodic branch will be much flatter than the cathodic branch. The reverse will also apply at pH's close to $\mathrm{pK}_{\mathrm{a} 1}$. Figure 1.13.2 and Figure1.13.3 are indicative of simulated CV's and Tafel plots under both $\mathrm{pH}$ conditions.

The apparent standard rate constant still has a strong dependence on $\mathrm{pH}$ in both systems. However, using the Finklea model, the system with the potential dependent transfer coefficient will show a more dramatic decrease in apparent rate as the $\mathrm{pH}$ approached $\mathrm{pK}_{\text {mid. }}$. Figure 1.13.4 shows the apparent rate vs. $\mathrm{pH}$ for both systems. Note that a lower reorganization energy results in a more pronounced deviation from the curve for $\alpha=0.5$.

\subsection{Direction and Goals of this Research Project}

This research project was designed to probe the thermodynamics and kinetics of PCET redox couples attached to self-assembled monolayers on electrodes. Specifically, the goals of this research are as follows:

1. Validate Laviron's approximations for the thermodynamics of PCET of attached redox systems $\left(E_{r}\right.$ vs $\left.p H\right)$. 


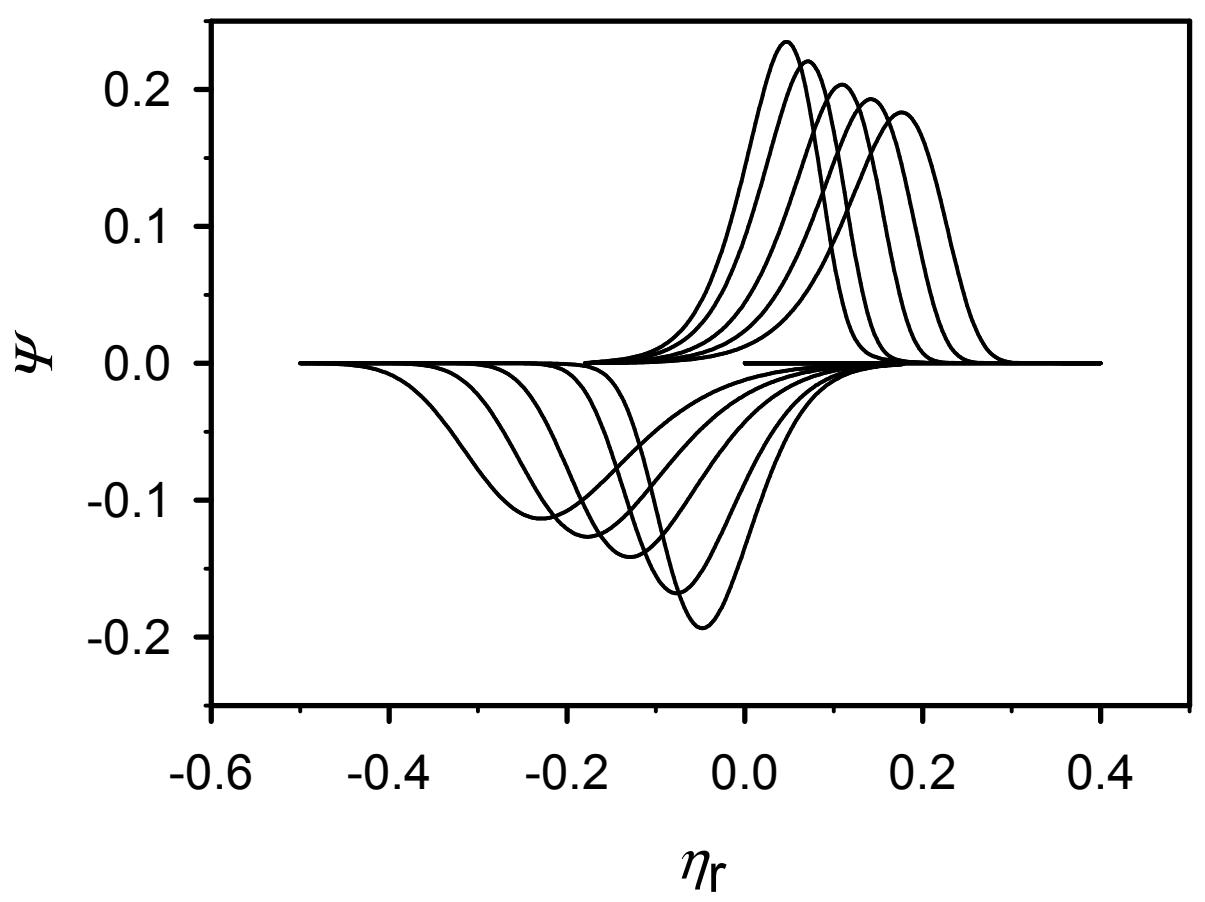

Figure 1.13. 2

Theoretical Voltammogram of $1 \mathrm{e} 1 \mathrm{H}$ system where $\mathrm{pK}_{\mathrm{a} 1}<\mathrm{pH}<\mathrm{pK}_{\text {mid. }} \psi$ represents the theoretical dimensionless current. Larger peak splittings are indicative of greater scan rates. 


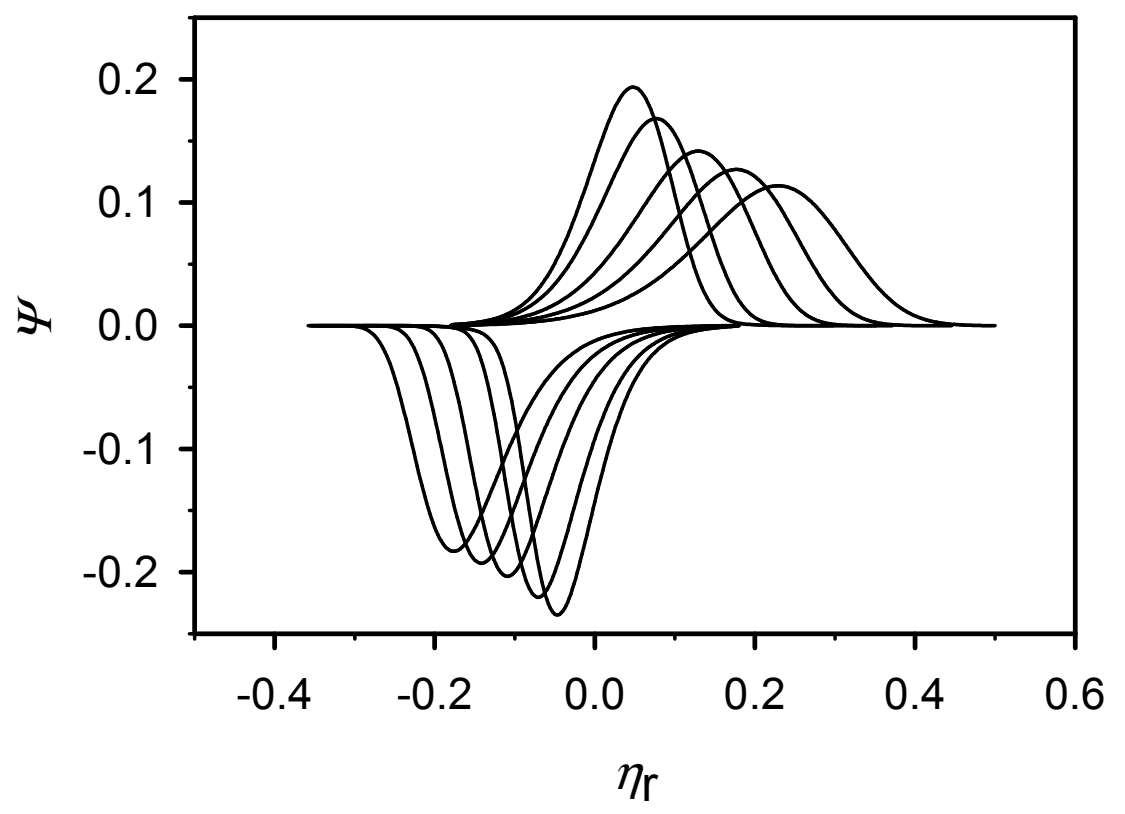

Figure 1.13.3 Theoretical voltammograms of $1 \mathrm{e} 1 \mathrm{H}$ system where $\mathrm{pK}_{\mathrm{a} 2}>\mathrm{pH}>\mathrm{pK}_{\text {mid }}{ }^{32} . \psi$ represents the theoretical dimensionless current. Larger peak splittings are indicative of greater scan rates. 


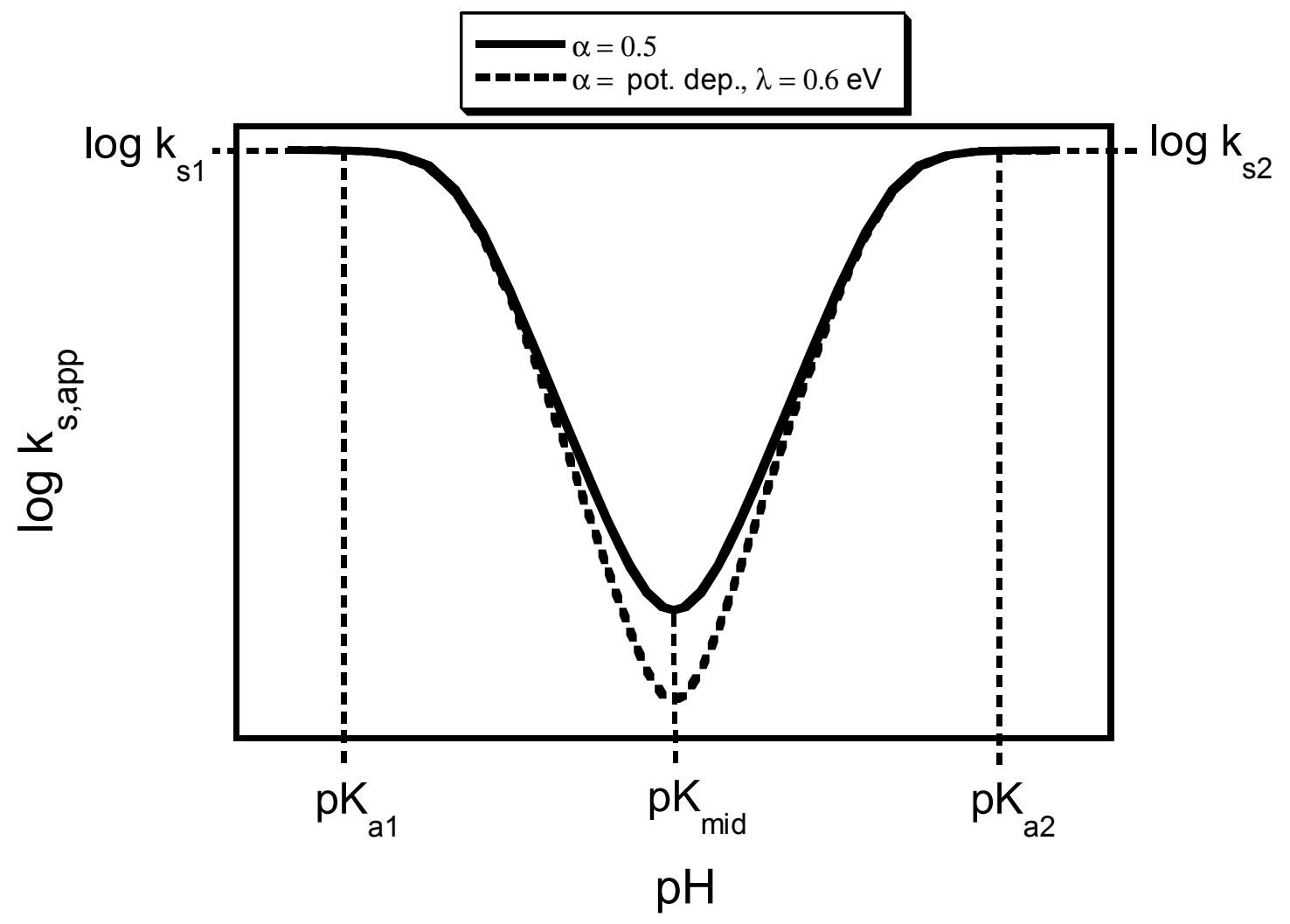

Figure 1.13. 4 Theoretical $\log \mathbf{k}_{\mathrm{s} \text {,app }}$ vs $\mathrm{pH}$ using the Finklea model 
2. Compare experimental results to theoretical Marcus DOS model to validate that it is an acceptable model for PCET of redox centers on electrodes, and that the transfer coefficients are dependent on potential.

3. Extract as many experimental parameters as possible $\left(E^{0}, p K_{a}, k_{s}, \lambda\right)$ from model systems.

These goals were be accomplished examination of the thermodynamics and kinetics of redox couples attached to self-assembled monolayers. We restricted analysis to simple 1-proton/1-electron systems. In particular, an organic system (galvinol/galvinoxyl) and an inorganic system ([Os(bpy) $\left.\left.)_{2}(4-\mathrm{AMP}) \mathrm{OH}_{2}\right]^{2+/ 3+}\right)$ were studied. For each system, scan rate studies were performed over a wide $\mathrm{pH}$ range. Each scan rate study consisted of multiple voltammograms taken over a range of scan rates. Both thermodynamic $\left(\mathrm{pK}_{\mathrm{a} 1}, \mathrm{pK}_{\mathrm{a} 2}, \mathrm{E}_{1}{ }^{0^{\prime}}, \mathrm{E}_{2}{ }^{0^{\prime}}\right)$ and kinetic $\left(\mathrm{k}_{\mathrm{s} 1}, \mathrm{k}_{\mathrm{s} 2}, \alpha_{1}, \alpha_{2}, \lambda_{1}, \lambda_{2}\right)$ parameters of each system were sought, although, in the case of the galvinol system, only high $\mathrm{pH}$ parameters were obtained. Experimental results were compared with the results predicted by Laviron and Finklea. 


\subsection{Instrumentation}

All electrochemical experiments were performed using either a Princeton Applied Research Model 173 or Model 283 Potentiostat coupled with a Princeton Applied Research Model 175 Analog Waveform Generator. A Princeton Applied Research Model 5210 lock-in analyzer (LIA) was used in conjunction with the potentiostat for AC measurements. Data was recorded on a Tektronix TDS430A digital oscilloscope. The potentiostat, LIA and oscilloscope were connected via GPIB interface to a Pentium model PC. The computer interface allowed remote control of the potentiostat and LIA, using Powersine software, for impedance analysis. It also provided direct transfer of all data from the oscilloscope to the $\mathrm{PC}$. $\mathrm{pH}$ measurements were taken using an Accumet $1003 \mathrm{pH}$ meter with a model 13-620-111 gel filled double junction combination electrode. All electrolytes were prepared using doubly distilled water that had been deionized using a Barnstead Mega-Pure purification system. Typical resistivities of the deionized water were greater than $16 \mathrm{M} \Omega-\mathrm{cm}$. All thermodynamic and kinetic data analysis was performed using a spreadsheet program designed on Microsoft Excel. 


\subsection{Cyclic Voltammetry}

Cyclic Voltammetry is the primary technique used to probe the thermodynamics and kinetics of the PCET systems in this dissertation. Cyclic voltammetry is a convenient technique in that it is both selective and sensitive. Coverages as low as $1 \times 10^{-12} \mathrm{~mol} / \mathrm{cm}^{2}$ can be successfully analyzed. In general, electroactive impurities can be readily spotted as separate or buried waves within a voltammogram. Cyclic Voltammetry provides a convenient means of separating the background (charging) current from the current involved in the electron transfer process (faradaic current), which is necessary prior to kinetic analysis. The Waveform Generator can provide scan rates as fast as $1000 \mathrm{~V} / \mathrm{s}$, which is necessary for the extraction of kinetic information.

\subsection{The Electrochemical Cell}

All experiments were performed in an electrochemical flow cell using a modified 3-electrode configuration. All experiments were performed at room temperature. A schematic of the cell is shown in Figure 2.3.1. The working (WKG) electrode was a polycrystalline gold bead. The counter electrode (CTR) was a platinum mesh electrode. The reference electrode (REF) was an Accumet 13-620-51 saturated calomel reference electrode (SCE). The fourth electrode was an Iridium-oxide quasireference electrode (IRREF) coupled in parallel with the SCE electrode to the potentiostat. A $10 \mu \mathrm{F}$ capacitor was placed between the Ir electrode and the parallel junction. The SCE provided a stable DC reference potential, 


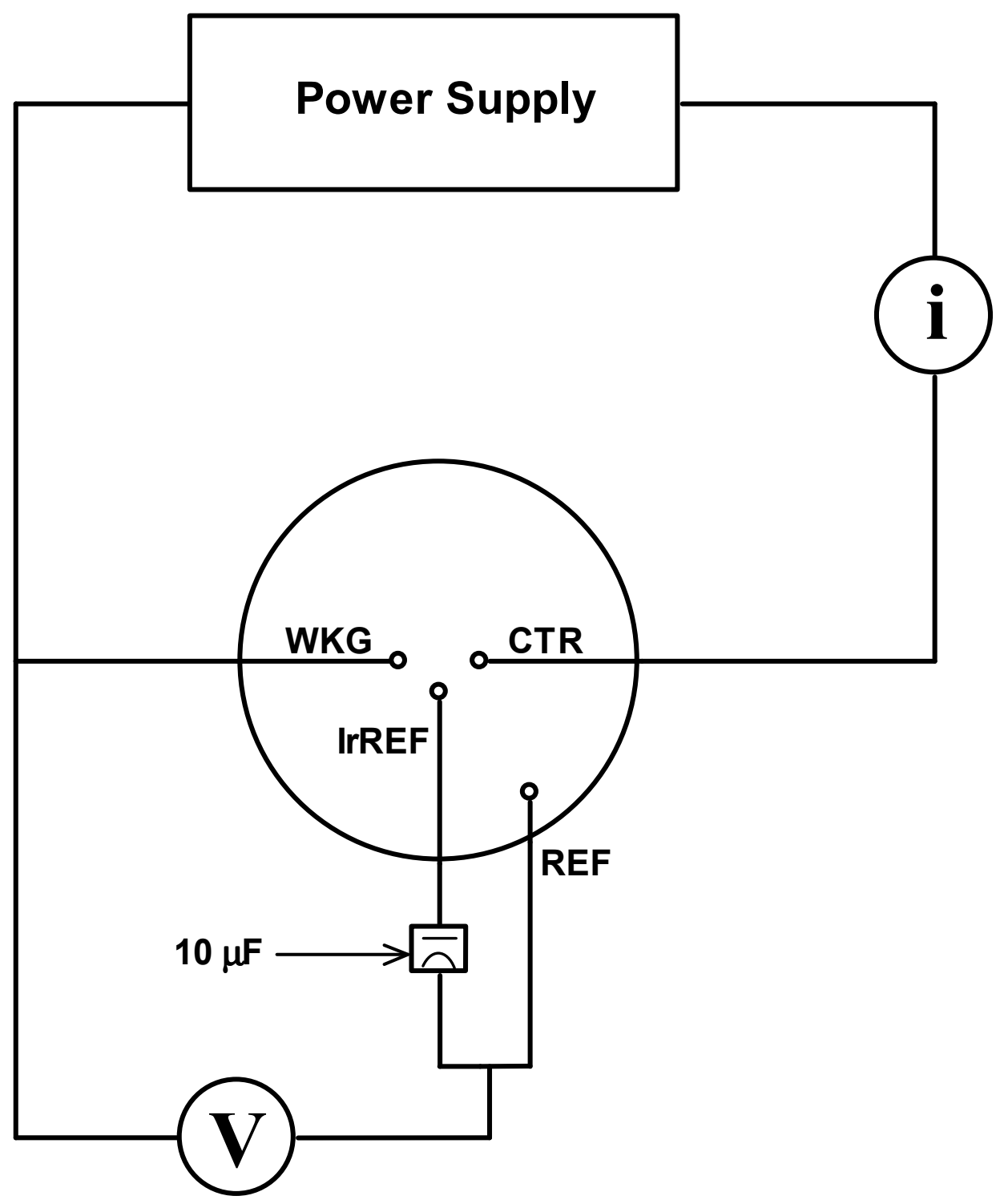

Figure 2.3. $1 \quad$ Cell Schematic 
while the IrREF provided a low impedance pathway for very fast transient measurements.

Cell design was a very important aspect in this research project. For reasons which will be discussed later, it was important to design a cell where the $\mathrm{pH}$ of the electrolyte could be changed rapidly. It was also necessary to design a cell that was effectively free of dissolved oxygen. The most important feature of the cell was that it must be able to provide quick changes of oxygen-free electrolyte without removing the electrode from the electrolyte solution and without turning off potential control by the potentiostat. The standard method for changing electrolyte in a standard 3 chamber cell required turning the cell off, removing the electrodes, flushing the cell with the new electrolyte and then reassembling the system. This technique was both time consuming (because of the number of electrolyte changes necessary) and disruptive to the redox centers on the working electrode. This problem was resolved with the design of the electrochemical flow cell seen in Figure 2.3.2. The flow cell consists of a central chamber, a reference electrode chamber, and a gravity-fed reservoir for changing the electrolyte. The central chamber houses the working, counter and Ir quasi-reference electrodes. The reference electrode chamber houses the saturated calomel reference electrode. The reservoir was separated from the central chamber by a Teflon stopcock, which allowed direct control of the flow of electrolyte from the reservoir. An overflow outlet was placed on the far side of the reference electrode chamber. Electrolyte was purged with nitrogen gas externally prior to placement in the reservoir. A Nitrogen gas bubbler was placed in the reservoir to ensure that the electrolyte 


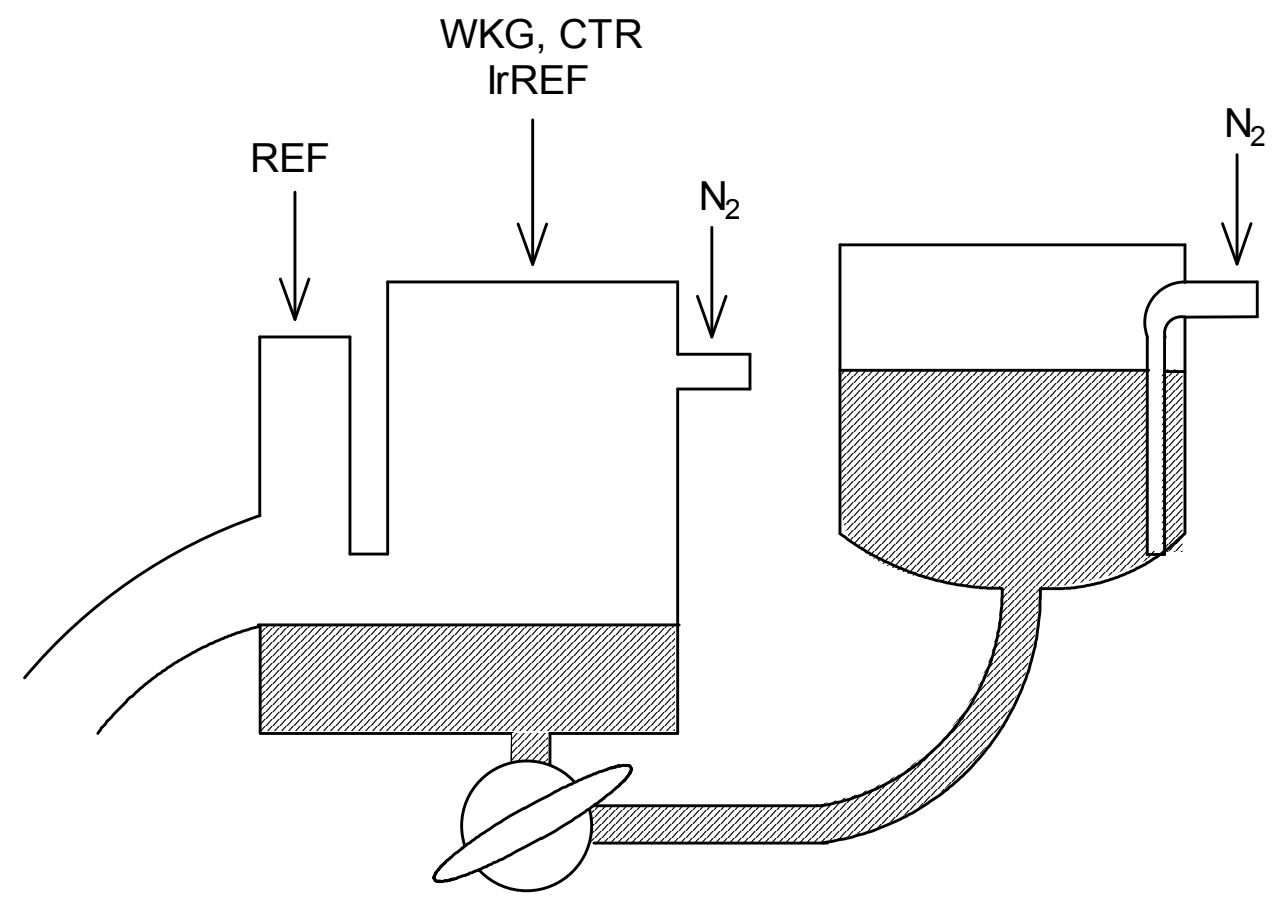

Figure 2.3. 2 The Electrochemical Flow Cell 
remained oxygen-free, and another inlet was placed above the solution in the central chamber to ensure that no oxygen seeped into the solution between flushes. There was still potential for oxygen to enter from the reference electrode cell, but this never occurred as long as sufficient gas was passed over the electrolyte. This cell design also allowed for a rapid change in electrolyte with a minimal volume of flushing solution. Typically complete flushing of the cell (where the $\mathrm{pH}$ of the central compartment was identical to the $\mathrm{pH}$ of the reservoir, regardless of initial $\mathrm{pH}$ of the central compartment) could be achieved in approximately 30 seconds with less than $100 \mathrm{ml}$ of solution.

By using the flow cell, $\mathrm{pH}$ changes could be made without disturbing the electrode, and without disturbing the electrical circuit. Because of the nature of electrolyte delivery, the electrode immersion depth increased during flushes, then returned to a constant level after each flush. This method is more consistent than the old method of visually estimating immersion depth each time the electrode is inserted into the electrolyte.

\subsection{The Electrodes - Synthesis, Cleaning, and Characterization}

The Reference Electrodes

The reference electrode used was a commercial standard SCE electrode. It was stored in dilute potassium chloride solution, and was rinsed with distilled, deionized water prior to use. 
The iridium oxide quasi reference electrode was created from a $.5 \mathrm{~mm}$ thick Iridium wire using a method modified from literature ${ }^{33}$. A $2.5 \mathrm{~cm}$ length of Iridium wire was placed in a crucible and covered with $50 \% \mathrm{w} / \mathrm{w}$ Sodium Hydroxide solution. The covered crucible was heated to glowing under a Bunsen burner flame for 20 minutes. This procedure formed an outer layer of iridium oxide without affecting the inner portion of the wire. Too much heating resulted in conversion on the entire wire, which was too brittle to be used. Immersion of this electrode in a flame resulted in loss of the oxide layer; thus it was simply rinsed with distilled, deionized water prior to each use. The Iridium oxide electrode was sensitive to hydrogen ion concentration, thus the IrREF potential was largely determined by $\mathrm{pH}$.

The Counter Electrode

A platinum mesh was used as the counter electrode. The mesh was suspended in the electrolyte using a platinum wire. Prior to use, the mesh was repeatedly rinsed with distilled, deionized water and heated until glowing in a Bunsen burner flame to clean off any residual contaminants.

The Working Electrode

Working electrodes were created from $0.5 \mathrm{~mm}$ gold wire. Approximately 1 inch lengths of wire were used for each electrode. A bead was formed on the end of each wire by melting the wire tip in a Bunsen burner flame. Beads of approximate area of $0.1 \mathrm{~cm}^{2}$ were created through careful manipulation of the wire tip in the flame. 
Gold bead electrodes were cleaned using two methods. For bare electrode voltammetry, the gold beads were repeatedly rinsed with distilled, deionized water and flamed (heated until they visibly glowed) using a Bunsen burner. In experiments where a monolayer was going to be deposited on the electrode, a second cleaning step was added. This second step was based on the formation and dissolution of an oxide from the surface of the electrode. First, the electrode was flamed using a Bunsen burner. Then the electrode was connected to a cell containing a dilute acid solution $(0.1 \mathrm{M}$ $\mathrm{H}_{2} \mathrm{SO}_{4}$ or $\mathrm{HClO}_{4}$ ). An initial CV was taken to assess the initial condition of the electrode. Then approximately $0.5 \mathrm{~cm}$ of the wire above the bead was immersed in the electrolyte. The electrode was galvanostatically oxidized (with an anodic current of around $30 \mathrm{~mA}$ ) for one minute. The potential of the electrode was approximately $+2.0 \mathrm{~V}$ vs SCE during this step. The electrode was then removed from the cell and dipped in $3 \mathrm{M} \mathrm{HCl}$, which removed the newly formed oxide layer. The electrode was rinsed in distilled, deionized water, and then assessed by cyclic voltammetry. A flat charging current with a sharp rise of anodic current at around $+1.0 \mathrm{~V}$ vs SCE followed by a sharp reduction at around $+0.75 \mathrm{~V}$ vs SCE is indicative of a properly cleaned electrode. The presence of unknown bumps in the voltammogram is due to impurities adsorbed to the electrode. This procedure served two purposes. First, stripping of the outer layer of gold removed any impurities adsorbed on or imbedded in the surface of the electrode. The stripping step also helped to remove imperfections in the polycrystalline lattice, which resulted in a more uniform surface to be used in monolayer deposition. A more 
uniform gold surface is believed to lead to more densely packed and uniform selfassembled monolayers.

\subsection{Monolayer Deposition, General Discussion}

This section discusses the general parameters for monolayer deposition, while subsequent sections will provide experimental details. Typically, a small amount of long-chain alkanethiol, or derivative thereof, is dissolved in about $10 \mathrm{ml}$ of absolute ethanol. An electrode is totally immersed into the ethanol for at least 30 minutes. Typically monolayers were deposited overnight. A monolayer will spontaneously form on the surface of the electrode. This self assembly occurs because of the affinity of the sulfur head group to the metal surface. This is quite a strong interaction; however, it is not known whether the interaction is purely electrostatic in nature, or whether the bond has some measure of covalency. Regardless, over relatively short periods of time the thiols will self assemble onto the electrode to form a tightly packed, well ordered system.

\subsection{Characterization of SAM's}

The primary purpose of a SAM is to hold the redox center at a fixed distance from the electrode while minimizing interaction between the free solution and the electrode. The insulating ability of a monolayer can be characterized by capacitance 
measurements. Capacitance is a measure of the charging current per unit area of electrode. The typical capacitance of a bare gold electrode in aqueous solution is around $20-100 \mu \mathrm{F} / \mathrm{cm}^{2}$. Deposition of a monolayer onto an electrode greatly reduces the capacitance, and consequently the charging current. Smaller capacitances correspond to more tightly packed monolayers. Typical capacitances of SAM coated electrodes can vary significantly, but are on the order of $0.5-10 \mu \mathrm{F} / \mathrm{cm}^{2}$. Experimentally, capacitance can be measured via the charging current, according to the following equation ${ }^{2}$ :

$$
\mathrm{C}=\frac{\mathrm{i}_{\mathrm{ch}, \mathrm{tot}}}{2 \mathrm{v}}
$$

where $C$ is the capacitance $(F), i_{c h, t o t}$ is the total charging current $(A)$, and $v$ is the scan rate $(\mathrm{V} / \mathrm{s})$. Another general issue that should be introduced is the potential window limitation of SAM coated gold electrode. The potential window of SAM-coated gold electrodes are limited mainly by three factors: gold oxidation, water reduction, and monolayer desorption. Desorption of the monolayer can occur at extreme negative potentials, typically more negative than $-1.0 \mathrm{~V}$ vs SCE in very basic solution. Care was taken to avoid potential extremes, since both gold oxidation (at high potentials) and monolayer desorption (at low potentials) disturb the packing of the monolayer (except at high scan rates, where the time constant of the scan is much faster than than the oxidation/reduction time constant). 


\subsection{Thermodynamic Characterization of Attached Redox Centers on SAM's}

There are two general methods for creating a redox center-attached SAM. The first method involves coupling the redox center to the distal end of a thiol chain prior to adsorption of the thiol to the electrode. The now electroactive SAM is then deposited onto the surface of the electrode. The second method involves depositing the SAM onto the electrode first, then coupling the monolayer to an electroactive center. Each method will be discussed in more detail later.

Redox centers attached to SAM's can be characterized in a number of ways. Prior to analysis, the nonfaradaic component to the current must be separated from the faradaic component. Figure 2.7.1 gives a visual description of this separation, which can be accomplished using baseline correction techniques. For either the anodic or cathodic peak, the coverage of the redox couple on the monolayer is defined as

$$
\Gamma=\frac{\mathrm{Q}_{\text {tot }}}{\mathrm{nFA}}
$$

where $\Gamma$ is the coverage $\left(\mathrm{mol} / \mathrm{cm}^{2}\right), \mathrm{n}$ is the number of electrons transferred, $\mathrm{F}$ is Faraday's constant, and A is the surface area of the electrode $\left(\mathrm{cm}^{2}\right)^{9} . Q_{\mathrm{tot}}$ is evaluated by integrating the area under the individual peaks

$$
\mathrm{Q}_{\mathrm{tot}}=\int \mathrm{i}_{\mathrm{f}} \mathrm{dt}
$$




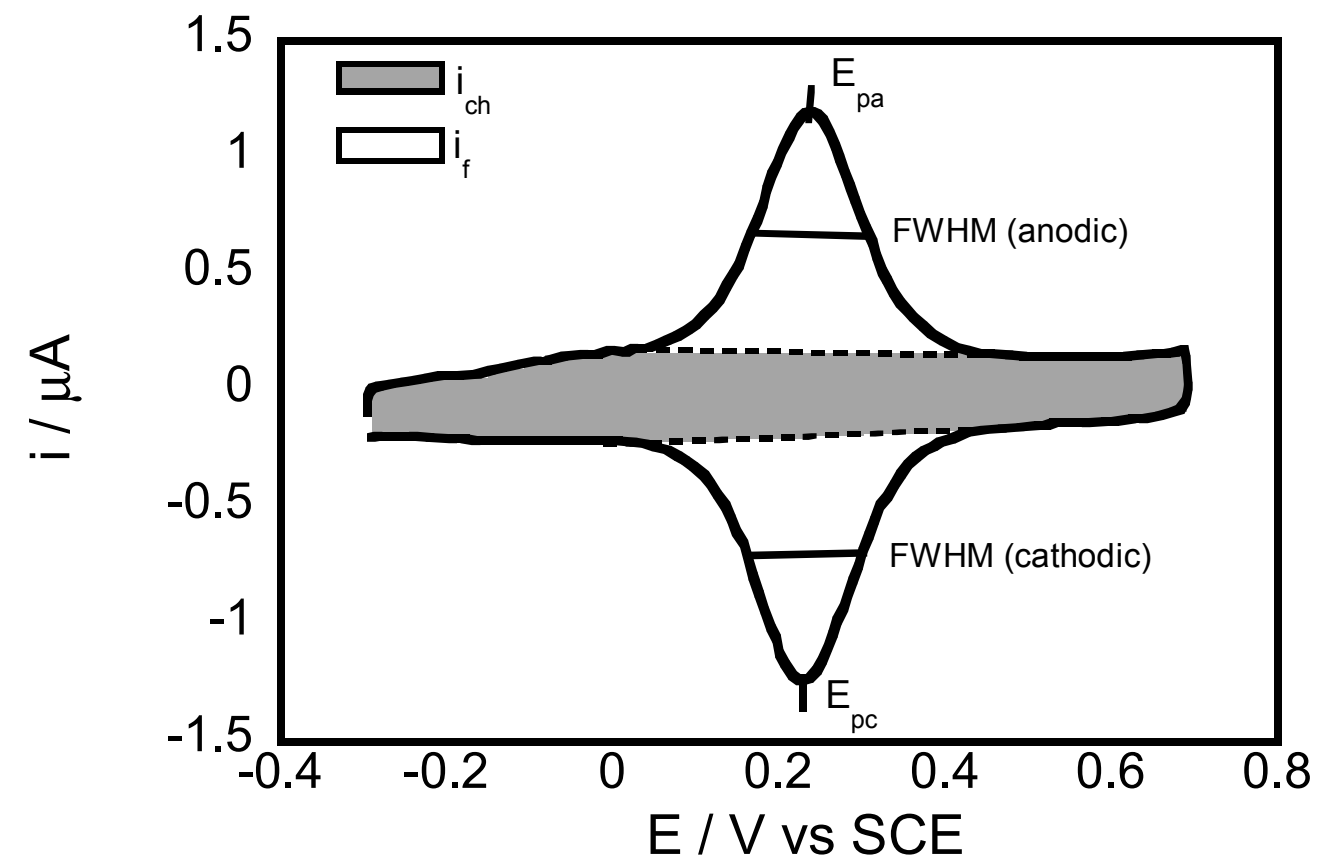

Figure 2.7. 1 Faradaic and Nonfaradaic components of a CV. $i_{c h}$ is nonfaradaic (charging) current and $i_{f}$ is faradaic current. $\Delta E_{p}$ is the average of $E_{p a}$ and $E_{p c}$. FWHM is the peak width at half the height of the peak. 
$Q_{\text {tot }}$ for the cathodic branch should equal $Q_{\text {tot }}$ for the anodic branch. The area of the electrode can be calculated by taking a bare electrode CV of a standard redox species of known concentration. $\mathrm{Ru}\left(\mathrm{NH}_{3}\right)_{3} \mathrm{Cl}_{3}$ was commonly used because it is a well characterized 1-electron system ${ }^{34 ; 35}$. Peak current $\left(i_{p}\right)$ of a redox species in free solution is defined as

$$
\mathrm{i}_{\mathrm{p}}=\left(2.69 \times 10^{5}\right) \mathrm{n}^{3 / 2} \mathrm{AD}_{0}^{3 / 2} \mathrm{v}^{1 / 2} \mathrm{C}_{0}^{*}
$$

$\mathrm{n}$ is the number of electrons transferred per redox couple, $\mathrm{D}_{0}$ is the diffusion coefficient (typically around $7 \times 10^{-6} \mathrm{~cm}^{2} / \mathrm{s}$ in aqueous solution), $v$ is the scan rate $(\mathrm{V} / \mathrm{s})$, and $\mathrm{C}_{0}{ }^{*}$ is the concentration of the redox couple in solution $\left(\mathrm{mol} / \mathrm{cm}^{3}\right)^{2}$. When a CV is under reversible conditions (at sufficiently slow scan rates), it is considered to be under thermodynamic control. Theoretically, reversible voltammograms of attached redox molecules elicit three main features. First, there should be no splitting between the cathodic and anodic waves $\left(\Delta \mathrm{E}_{\mathrm{p}}=0\right)$, and both peak potentials should lie at the formal potential $\left(E^{0^{\prime}}\right)$ of the redox molecule. Also, the peak half-width, $E_{f w h m}$, should equal 3.5RT/nF $(91 \mathrm{mV} / \mathrm{n})$ at room temperature ${ }^{2}$. Generally, peak shapes should also be symmetrical around the formal potential, and anodic and cathodic peaks should be mirror images across the current axis. In practice, conditions are considered reversible when peak splittings are small $\left(\Delta \mathrm{E}_{\mathrm{p}}<10 \mathrm{mV}\right)$. The formal potential is defined as

$$
\mathrm{E}^{0^{\prime}}=\frac{\mathrm{E}_{\mathrm{p}, \mathrm{c}}+\mathrm{E}_{\mathrm{p}, \mathrm{a}}}{2}
$$


where $E_{p, x}$ is defined as the peak potential for the cathodic and anodic waves. Well behaved systems tend to have peak half-widths of $100 \mathrm{mV}$ or less. Figure 2.7.1 is a good example of a voltammogram under reversible conditions. Note the small peak splittings, near-ideal peak half-width, and symmetry between the cathodic and anodic peaks. The deviation to non-zero peak splittings is not well understood, but attributed to ion-pair interactions with the electrolyte ${ }^{36}$. Deviations in ideal peak-widths can be attributed to several factors. The presence of multiple formal potentials (thermodynamic heterogeneity) will cause the peak shapes to broaden, resulting in higher peak half-widths. Thermodynamic heterogeneity is attributed to imperfections in the redox-attached SAM. Redox centers can be partially buried in the monolayer, which can change their interaction with the electrolyte. Since the thermodynamics of a redox center is highly dependent on $\mathrm{pH}$ in a PCET system, a distribution of formal potentials can result. Double layer effects are caused by changes in the local electrostatic potential of the redox centers during oxidation/reduction; these effects can result in broader CV peaks ${ }^{37}$.

In order to minimize double layer effects, coverages of the redox couple are intentionally kept low relative to the maximum coverage. Typical coverages are on the order of $1 \times 10^{-11} \mathrm{~mol} / \mathrm{cm}^{2}$ (vs. maximum coverages of $2-4 \times 10^{-10} \mathrm{~mol} / \mathrm{cm}^{2}$ ). Creating SAM's with a specific, reproducible coverage can be difficult. Three methods are used in this research to ensure that coverages are low enough for analysis. The primary method used to regulate coverage is to use a mixture of electroactive and nonelectroactive thiols to form the monolayer. Through self-assembly, it is assumed 
that the redox centers will attach themselves as far away from each other as possible, allowing the diluent thiol to fill in the gaps. The equilibrium coverage is dependent on the molar ratio of electroactive thiol vs. diluent thiol and the overall solution concentration ${ }^{38-41}$. Increasing the molar fraction of diluent thiol results in a decrease in coverage of redox thiols. The second method involves annealing an electrode that is coated with an electroactive monolayer. An electrode can be immersed in a solution that contains only diluent thiol. The diluent thiol will slowly exchange with some of the electroactive thiol. Regulation of deposition time and diluent thiol concentration allows significant control in lowering the coverage. The third method involves changing the deposition conditions and length of deposition. Particularly important when trying to couple a redox species to an existing monolayer, changing the deposition conditions can have a dramatic effect on monolayer formation. Lowering the temperature (which slows down the coupling reaction) and reducing the time of deposition will both reduce the final coverage of redox center on the monolayer. It is common to use a combination of some or all of these techniques in order to yield a reproducible low coverage monolayer.

\subsection{Kinetic Characterization of Redox Centers on SAM's}

We used two methods to explore the kinetics of redox-attached SAM's. The first method is based on the calculation of the apparent standard rate constant, $\mathrm{k}_{\mathrm{s}, \mathrm{app}}$ at zero overpotential. The current at any overpotential, $\eta$, is defined as 


$$
\mathrm{i}_{\mathrm{f}}(\eta)=\left(\mathrm{k}_{\mathrm{c}, \mathrm{app}}+\mathrm{k}_{\mathrm{a}, \mathrm{app}}\right) \mathrm{Q}_{\text {tot }}\left[f(\eta)-f_{\text {Nernst }}(\eta)\right]
$$

where $\mathrm{i}_{\mathrm{f}}(\eta)$ is the faradaic current, $f(\eta)$ is the actual fraction of reactant charge remaining, and $f_{\text {Nernst }}(\eta)$ is the theoretical fraction of reactant charge remaining as defined by the Nernst equation ${ }^{32}$. At zero overpotential, $\eta=0, \mathrm{k}_{\mathrm{s}, \mathrm{app}}=\mathrm{k}_{\mathrm{a}, \mathrm{app}}=\mathrm{k}_{\mathrm{c}, \mathrm{app}}$ and $f_{\text {Nernst }}(\eta)=0.5$. Thus, eq (2.8.1) simplifies to

$$
\mathrm{k}_{\mathrm{s}, \mathrm{app}}=\frac{\left(\frac{\mathrm{i}_{\mathrm{f}}(0)}{\mathrm{Q}_{\text {tot }}}\right)}{2 f(0)-1}
$$

Typically this analysis is valid at moderate peak splittings, $20-100 \mathrm{mV}$, where $\mathrm{f}(0)<$ 0.95. At small peak splittings, $(2 \mathrm{f}(0)-1)$ becomes small and uncertain. At very large peak splittings, $\mathrm{i}_{\mathrm{f}}(0)$ becomes small and uncertain.

The second method for extracting kinetic information from a CV uses Tafel analysis. In Tafel analysis, $\eta$ and $\log (k)$ values are plotted for both the anodic and cathodic waves. The anodic and cathodic branches can be extrapolated to $\eta=0$ to provide a value for $\mathrm{k}_{\mathrm{s}, \mathrm{app}}$. In order to maximize faradaic contribution and minimize problems associated with potential impurities, data is extracted at the point in each CV where $50 \%$ of the reactant has reacted ( $50 \%$ conversion). The rate constant is calculated using eq (2.8.1). The value of $\eta$ where $f(\eta)=0.5$ is located and data at that point are used to calculate $\mathrm{k}(50 \%)$. Each scan rate yields a pair of points on the Tafel plot. As the scan rate increases, $\eta(50 \%)$ moves to larger overpotentials. If a Tafel plot has significant curvature, the reorganization energy can be obtained. The curvature of 
the Tafel plot is fitted to theoretical Tafel plots of various reorganization energies. The optimum reorganization energy is based on the lowest sum of the squared errors between the data and the theoretical curve. The fit also yields $k_{s}$. In Tafel plots with no visible curvature, the transfer coefficient at zero overpotential can be approximated using the Butler-Volmer approach. Log k vs $\eta$ are fitted to a linear regression line. Again, the intercept at $\eta=0$ yields $k_{s}$.

\section{$2.9 \quad$ iR Drop and Uncompensated Resistance}

$\mathrm{iR}$ drop is a concern in all aspects of voltammetry. iR drop is defined as the ohmic potential drop between the working electrode and reference electrodes. A general schematic of an electrochemical cell is seen in Figure 2.9.1. iR drop is introduced because there is a finite resistance associated with the solution between the WKG and REF electrode, which is dependent on a number of factors, including electrolyte composition and distance between electrodes. This resistance is called uncompensated resistance, $R_{u}$ (because its contribution to overall cell resistance is not compensated by the potentiostat). The main consequence of this occurrence is that any true overpotential calculation is skewed by this contribution according to the following equation

$$
\eta(t)=E_{a p p l}-E^{0^{\prime}}-i(t) R_{u}
$$




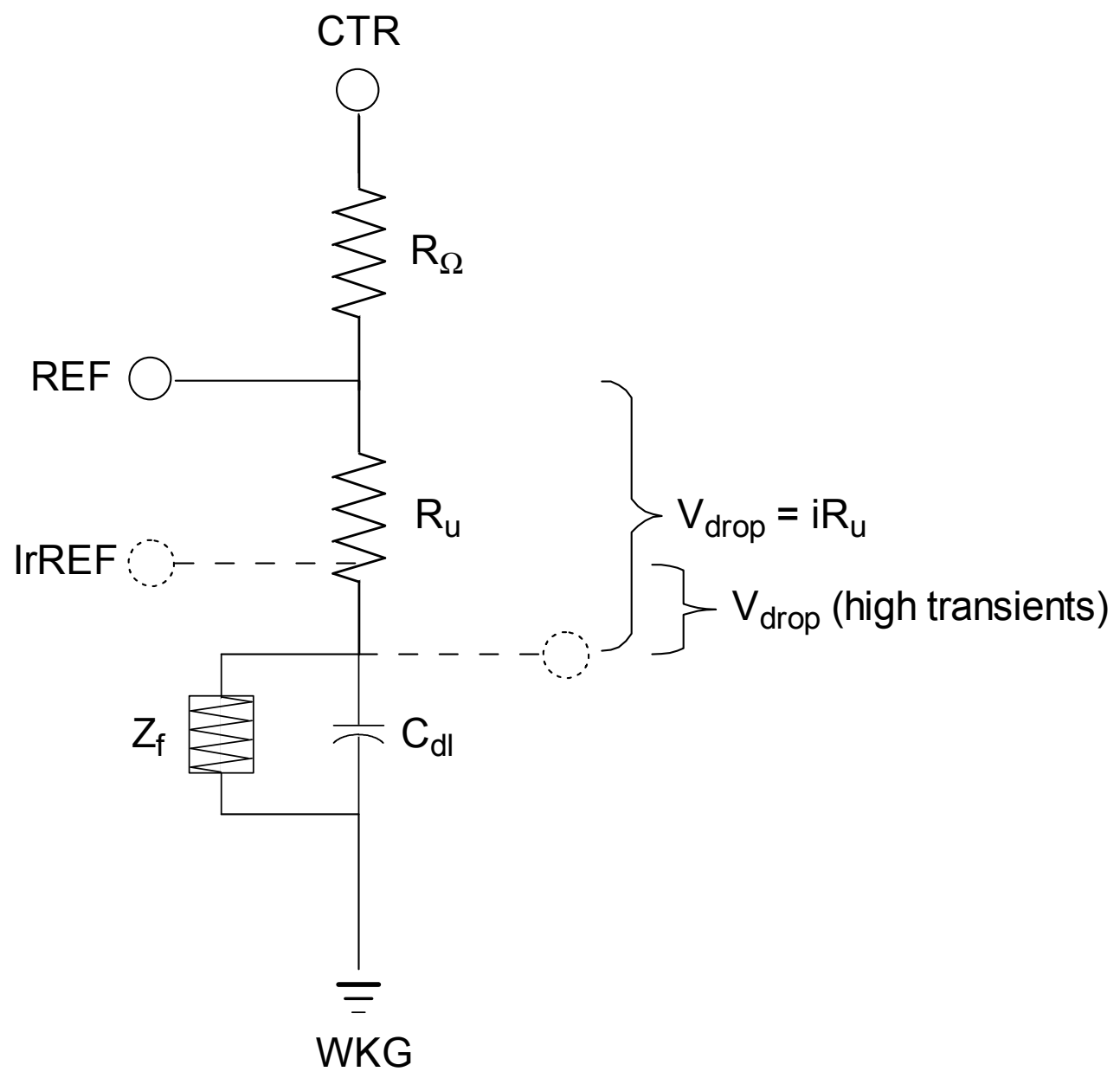

Figure 2.9. 1 RC Circuit showing uncompensated resistance component 
where $E_{\text {appl }}$ is the applied potential, and $i(t)$ is the total current at time t.. Since iR drop is a systematic error, it can be routinely corrected during data analysis. It is important to note that iR drop is only considered significant when it exceeds about $10 \mathrm{mV}$. There are a couple of techniques used to reduce iR drop. The most common technique is to simply increase the ionic strength of the electrolyte, which will decrease the solution resistance. An alternate method is to move the REF electrode closer to the WKG electrode. This method is currently employed in our electrochemical cell setup. The Ir quasi-reference electrode is placed very close to the WKG electrode. At high scan rates (where iR drop could be significant) the Ir electrode provides a low impedance pathway, which effectively moves the reference electrode closer to the WKG electrode while still using the DC reference potential generated from the SCE reference electrode.

In order to compensate for iR drop during data analysis, the uncompensated resistance of the cell needs to be known. The uncompensated resistance of a cell is readily determined using Impedance spectroscopy. Referring back to the equivalent circuit diagram, Figure 2.9.1, one can see that, at high frequencies, the low impedance of the interfacial capacitance shorts out the faradaic impedance, $Z_{\mathrm{f}}$. At sufficiently high frequencies, the total cell impedance is just the uncompensated resistance. In this project, two methods for calculating $R_{u}$ are used, one using manual data collection, the other using automated data collection via computer interface. Both methods are based on the same principle. 
The automated method uses the 5210 LIA coupled with the 283 potentiostat. The instruments are computer controlled via a GPIB interface by Powersine software, a commercial AC Impedance Spectroscopy software package. The DC potential is defined by the user, and should be at a position with no significant faradaic current. Cell impedance is measured over a range of frequencies (typically $10-100 \mathrm{KHz}$ ). At these high frequencies, the in-phase cell impedance converges to $R_{u}$.

The manual data collection method was used when the 283 potentiostat was not available for use. Instead, the 5210 LIA was coupled with the 173 potentiostat. Unfortunately, the 173 does not allow computer control, so all data was collected manually. Again impedance data was collected at a potential away from any faradaic processes at high frequencies $(>10 \mathrm{kHz})$.

\subsection{Excel Spreadsheet Analysis of Kinetics of SAM}

This section will provide detailed information on the kinetic analysis of a redox species attached to a SAM. The development of an integrated data analysis program was essential in completing this research project in a timely manner. In a typical study, at least 15 voltammograms at varying scan rates must be collected and analyzed for a monolayer in a given electrolyte. There are a number of steps are involved in analyzing the thermodynamic properties of a voltammogram. First, background correction techniques must be used to remove the charging current from the

voltammogram. The $\mathrm{CV}$ must be corrected for $\mathrm{iR}$ drop prior to any analysis. $\mathrm{E}_{\mathrm{p}, \mathrm{a}}$ and 
$E_{p, c}$ must be extracted from the voltammogram and $\Delta E_{p}$ must be calculated. Where $\Delta E_{p}$ is small (50 mV) $E_{p, a}$ and $E_{p, c}$ must be averaged to provide a value for $E^{0^{\prime}}$. For each $\mathrm{CV}$, peak currents, the current must be integrated to provide $Q_{\text {tot. }}$. The fraction of charge remaining, (f), must be calculated at each $\eta$. All of these steps can be automated with the exception of background correction and extraction of $\mathrm{E}_{\mathrm{p}, \mathrm{a}}$ and $\mathrm{E}_{\mathrm{p}, \mathrm{c}}$. Kinetic analysis must also be performed using spreadsheet analysis. For Tafel analysis, $\eta$ and $\mathrm{k}(\mathrm{t})$ values must be extracted at the point of $50 \%$ conversion (where $50 \%$ of the redox couples have been oxidized/reduced). This procedure is completely automated. The data from all CV's were then combined into Tafel plots. The other analysis procedure was based on calculating the standard rate constant, $\mathrm{k}_{\mathrm{s}}$, at zero overpotential. Current and charge fraction measurements at zero overpotential, $\mathrm{i}_{\mathrm{f}}(0)$ and $f(0)$, were extracted from the data and $k_{s}$ was calculated. The $k_{s}$ values for the anodic and cathodic peaks were averaged to yield an average apparent rate, $\mathrm{k}_{\mathrm{s}, \mathrm{app}}$. This analysis was also largely automated. 


\section{Chapter 3 Proton Coupled Electron Transfer Kinetic Studies of Galvinol}

\subsection{Introduction to Galvinol}

Galvinol/galvinoxyl is one of a group of organic molecules called phenoxyl radicals. Galvinol refers to the reduced, protonated form and galvinoxyl refers to the oxidized, deprotonated, radical form. Phenoxyl radicals (especially those with steric blocking groups to protect the reactive ortho sites) are interesting because they can often exist in solution as stable radicals ${ }^{42-44}$. Galvinoxyl is a phenoxyl radical with one radical site that is surrounded by blocking t-butyl groups, see Figure 3.1.1, which provides it excellent stability in aqueous solution. Currently, galvinol research is taking place along a number of fronts. The most prominent area of current research interest lies primarily in the area of molecular magnets. Crystalline galvinoxyl shows strong ferromagnetic tendencies ${ }^{45}$. As ferromagnetism is a "bulk" property, significant magnetic properties require the organization of a large number of paramagnetic units. Through monolayer self-assembly, an ordered system can potentially be developed using galvinol-thiols as building blocks ${ }^{46}$. There are numerous papers using the galvinoxyl radical in combination with metallic centers to probe the potential of organic and molecular magnetism ${ }^{38: 45-49}$. Galvinoxyl is also commonly used in food science as a determinant of the antioxidant potency of various consumable food products ${ }^{50 ; 51}$. The radical scavenging ability of potential antioxidants is determined by measuring the reduction of 

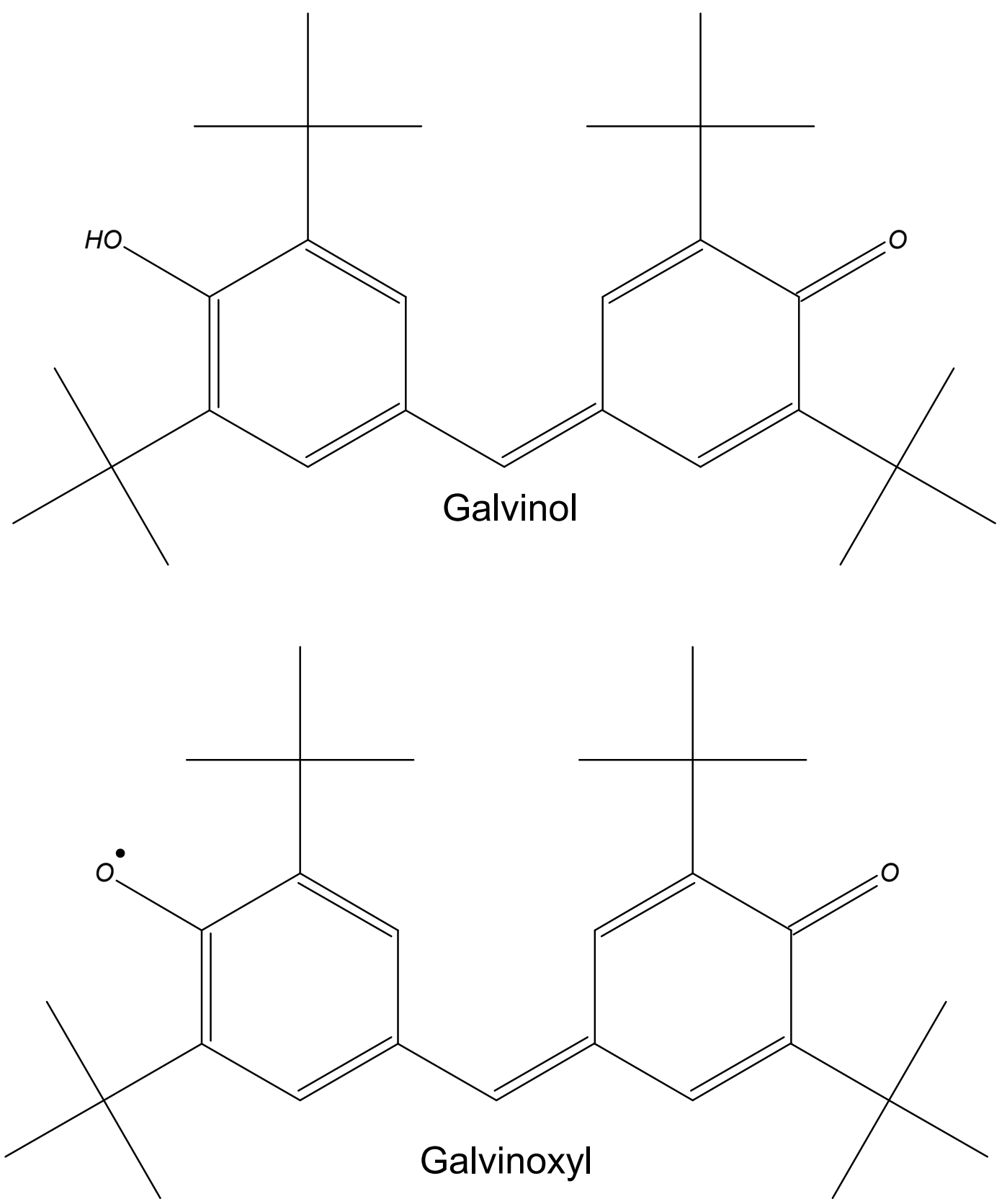

Figure 3.1. 1 The Galvinol/Galvinoxyl System 
radical concentration (usually by electron spin resonance) upon the addition of the antioxidant in both water-soluble and lipid-soluble mediums.

The galvinol/galvinoxyl system came to our attention in a publication by Prof. David Shultz, from NC State University ${ }^{45}$. In his article, a phenyl-galvinol terminated alkanethiol was successfully synthesized and attached to an electrode to form a well organized monolayer (the galvinol is attached to a phenyl-terminated thiol through the bridging carbon). This was interesting for a number of reasons. First, it showed that the galvinol/galvinoxyl radical could successfully be attached to a thiol monolayer and adsorbed onto an electrode. The monolayer was stable in aqueous solution over a reasonable $\mathrm{pH}$ range (5-10). More importantly, this paper reported that the formal potential was directly dependent on the $\mathrm{pH}$ of the solution. The $\mathrm{pH}$ dependence was Nernstian, with a slope of $-0.060 \mathrm{~V} / \mathrm{pH}$ unit. This $\mathrm{pH}$ dependency, coupled with the 1electron radical exchange, was consistent with the behavior of a 1e1H system. The subsequent publication of articles by Shultz confirmed this behavior ${ }^{38 ; 46}$. According to the publication, the galvinol-thiol became unstable at $5>\mathrm{pH}>10$, with loss of faradaic current outside the stable range. Regardless, the galvinol system was of interest because it appeared that through study of the galvinol system, both thermodynamic and kinetic information on a $1 \mathrm{e} 1 \mathrm{H}$ system could be gained within a region of intermediate $\mathrm{pH}$. Thus the validity of Prof. Finklea's predictions based on a potential dependent transfer coefficient for a 1e1H system could be evaluated.

Through correspondence with Prof. Shultz, Prof. Finklea obtained a small amount ( $<60 \mathrm{mg}$ ) of $\mathrm{HS}\left(\mathrm{CH}_{2}\right)_{10} \mathrm{O}$-phenyl-galvinol (hence referred to as galvinol-thiol). 
The following sections will discuss the characterization of the galvinol-thiol, along with the exploration of the thermodynamics and kinetics of the system, followed by a discussion of the galvinol system as it applies to proton coupled electron transfer.

\subsection{Experimental}

The alkanethiol terminated galvinol was supplied by Dr. David Shultz from North Carolina State University. 1-nonanethiol was prepared from 1-bromononane using a standard procedure from the literature ${ }^{52}$. The yield was $75 \%$ and the NMR of the distilled product is shown in Figure 3.2.1. 1-nonanethiol was chosen as the diluent thiol because it was slightly shorter than the alkanethiol tether, which ensured that the galvinol moiety was suspended outside the tightly packed alkanethiol domain. All buffer components were used without further purification.

A number of different buffer compositions were used in our electrochemical experiments. All buffers were calibrated using Fisher standard solutions at $\mathrm{pH} 4,7$, and 10. Early voltammetric experiments with galvinol used a complex electrolyte buffering system. Table 3.2.1 is a table listing the various buffer compositions used in preliminary experimentation. Buffers were prepared using sodium salts, with the exception of the $\mathrm{pH} 13$ buffer, where TMAH was used to minimize the alkaline error associated with the sodium cation. To simplify preparation, later experiments used a 


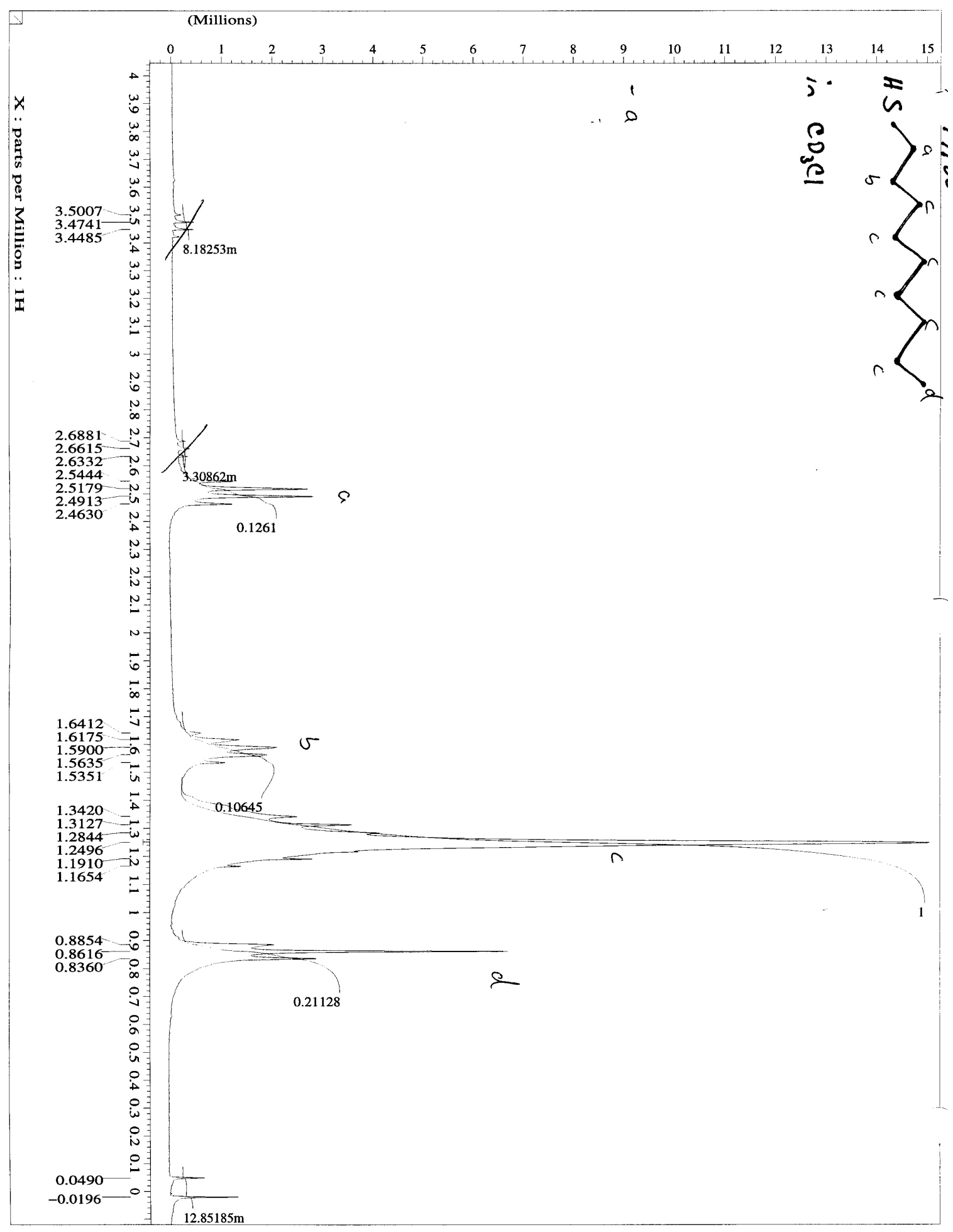

Figure 3.2. $1 \quad$ NMR of distilled 1-nonanethiol 
Table 3.2. $1 \quad$ Table of Electrolyte buffers

\begin{tabular}{|c||c|}
\hline pH & Electrolyte Buffer \\
\hline 2 & $0.1 \mathrm{M}$ phosphoric acid \\
\hline 3 & $0.1 \mathrm{M}$ phosphate buffer \\
\hline $4-5$ & $0.1 \mathrm{M}$ acetate buffer \\
\hline $6-8$ & $0.1 \mathrm{M}$ phosphate buffer \\
\hline $9-10$ & $0.1 \mathrm{M}$ borate buffer \\
\hline $11-12$ & $0.1 \mathrm{M}$ phosphate buffer \\
\hline 13 & $0.1 \mathrm{M}$ tetramethylamonium hydroxide \\
(TMAH)
\end{tabular}


single buffer system. This system consisted of $0.1 \mathrm{M}$ Britton-Robinson (B-R) buffer and $0.5 \mathrm{M} \mathrm{K}_{2} \mathrm{SO}_{4}$. B-R buffer was composed of equal concentrations of phosphate, borate, and citrate buffers. $\mathrm{K}_{2} \mathrm{SO}_{4}$ was added to control the ionic strength over the entire $\mathrm{pH}$ range. The $\mathrm{pH}$ of the buffer solution was adjusted with concentrated $\mathrm{H}_{2} \mathrm{SO}_{4}$ or $\mathrm{KOH}$, as necessary. Potassium salts were used instead of sodium salts in order to minimize the possibility of alkaline error, with the exception of the citrate salt, where only sodium citrate was available.

Mixed monolayers of the galvinol were prepared on gold beads. The freshly cleaned electrodes (see Section $\mathbf{2 . 4}$ for the procedure) were immersed in $10 \mathrm{ml}$ of absolute ethanol that contained $2.5 \mathrm{mg}$ each of 1-nonanethiol and galvinol thiol. Typically monolayers were deposited overnight (>12 hrs) and were subsequently annealed in a dilute solution of 1-nonanethiol in absolute ethanol, prior to use. The annealing step served two purposes. First, annealing in the diluent thiol improved the monolayer packing as shown by a decrease in interfacial capacitance. More importantly, the galvinol thiol slowly exchanged with the diluent thiol, which lowered the coverage of the galvinol on the monolayer. Low galvinol coverages were preferred in order to minimize possible interactions between individual galvinol couples. Low coverages also minimized systematic error in measurements due to uncompensated iR drop. Coverages of $1.0-2.0 \times 10^{-11} \mathrm{~mol} / \mathrm{cm}^{3}$ were selected as the minimum coverages which yielded adequate faradaic current for data analysis. This coverage is approximately one-tenth of the maximum coverage of $1.4-2.0 \times 10^{-10} \mathrm{~mol} / \mathrm{cm}^{2}$, as previously reported. 
Experiments were performed in a modified two-compartment electrochemical cell, as described in Section 2.3. It is important to note that the described cell was developed throughout this part of the project. The need for quick $\mathrm{pH}$ changes coupled to stability problems associated with the galvinol monolayer provided ample motivation for its development. Details on these problems will be discussed later.

Cyclic voltammetry was performed primarily using a Princeton Applied Research Model 283 potentiostat coupled with a Model 175 waveform generator. In the course of the project, there were times the Model 283 potentiostat was not available. During these times, a PAR Model 173 potentiostat was used in its place. Uncompensated resistance measurements were collected as described in Section 2.8, using the automated method when the Model 283 was available, and collecting the data manually when only the Model 173 was available. Typical uncompensated resistances ranged from 90-400 $\Omega$ when using the complicated buffer system, and 10-40 $\Omega$ when using the B-R buffer system. Uncompensated resistances were lower when using the B-R buffer because of the higher ionic strength from the added $\mathrm{K}_{2} \mathrm{SO}_{4}$.

Cyclic voltammograms of the galvinol monolayers were collected over a $\mathrm{pH}$ range of 2-14. Scan rate studies were performed on the galvinol monolayers over a pH range of 4-14. In initial experiments, voltammograms were limited to a $\mathrm{pH}$ range of 413 and scan rate studies were limited to a pH range of 7-13. Later experiments, using the B-R buffer along with a more rigorous degassing procedure, allowed data collection at lower $\mathrm{pH}$. 
Kinetic analyses were performed after isolating the faradaic current from the charging current and correcting the voltammograms for iR drop. Standard rate constants were obtained from voltammograms exhibiting intermediate peak splittings (20-200 mV) as discussed in Chapter 2. Tafel data (log (k) vs. $\eta$, where $\left.\eta=E-E^{0}\right)$ were also extracted at the time corresponding to $50 \%$ conversion of the reactant to the product on the anodic and cathodic faradaic peaks. The selection of $50 \%$ conversion is designed to minimize error associated with isolation of the faradaic current by extrapolation and subtraction of the charging current.

\subsection{Results - Preliminary Data}

Initially, data was collected to provide a general assessment of the galvinol system. Voltammograms and scan rate studies were collected using multiple monolayers and multiple electrodes over as wide a $\mathrm{pH}$ range as possible. Electrolyte buffer composition varied depending upon $\mathrm{pH}$ as described in Table 3.2.1. The use of the modified 2 compartment cell allowed quick change of the $\mathrm{pH}$ by flushing with new solution. Coverages of the redox couples on the electrodes averaged $1.6 \times 10^{-11}$ $\mathrm{mol} / \mathrm{cm}^{2}$. Figure 3.3.1 is representative of reversible voltammograms over a $\mathrm{pH}$ range of 4-13. Voltammograms were collected at $0.1 \mathrm{~V} / \mathrm{s}$ over all pHs. Note that the CV's exhibit reversible behavior at all but the lowest pHs. Peak splittings are less than 20 $\mathrm{mV}$, and $\mathrm{FWHM}$ is around $100 \mathrm{mV}$. At $\mathrm{pH}<5$, peak splittings increase due to the slower kinetics associated with galvinol at low $\mathrm{pH}$. Thus apparent formal potentials can 


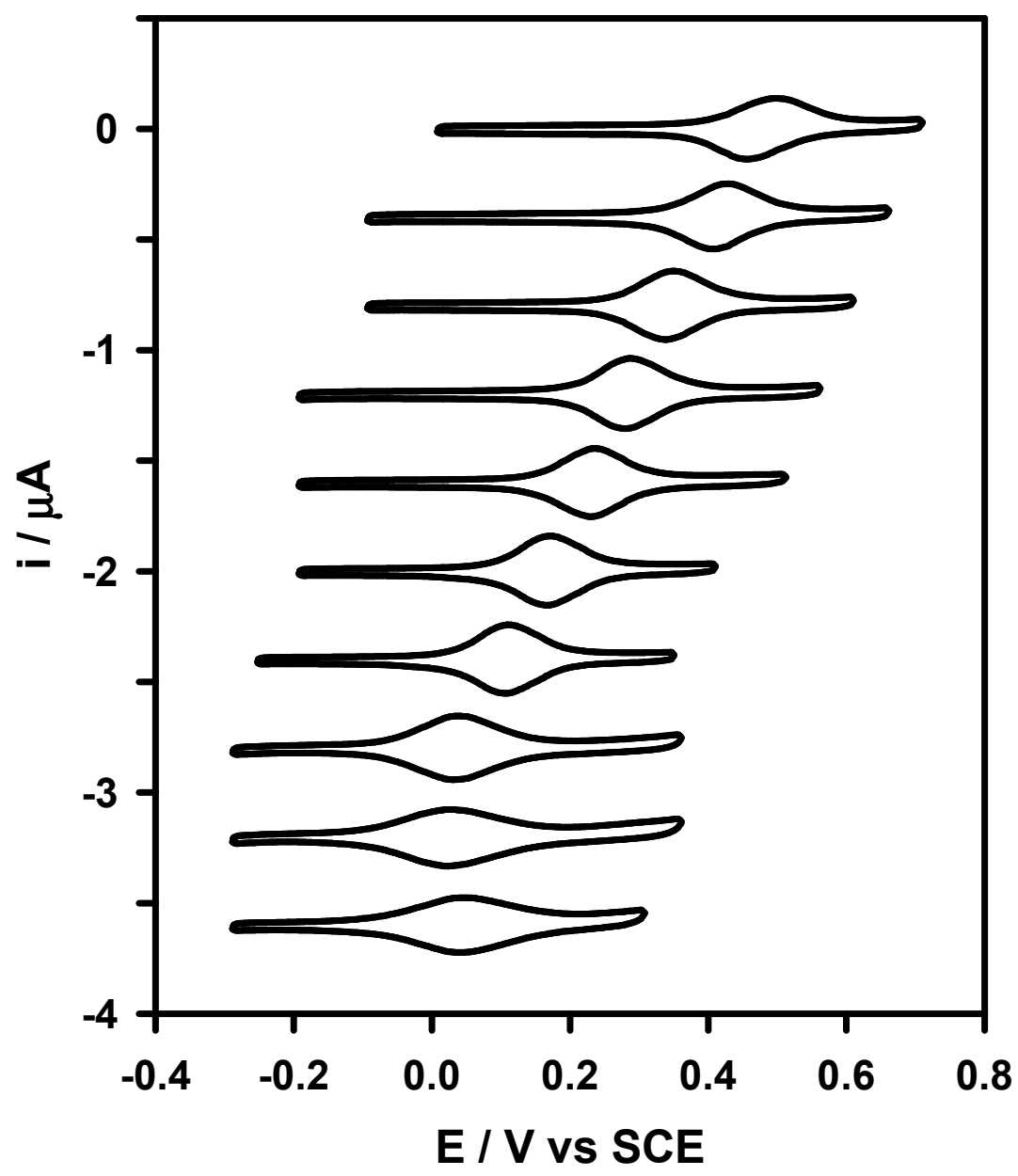

Figure 3.3. 1 Reversible Voltammograms $-\mathrm{pH} 4$ (top) to 13 (bottom) ${ }^{32}$. 


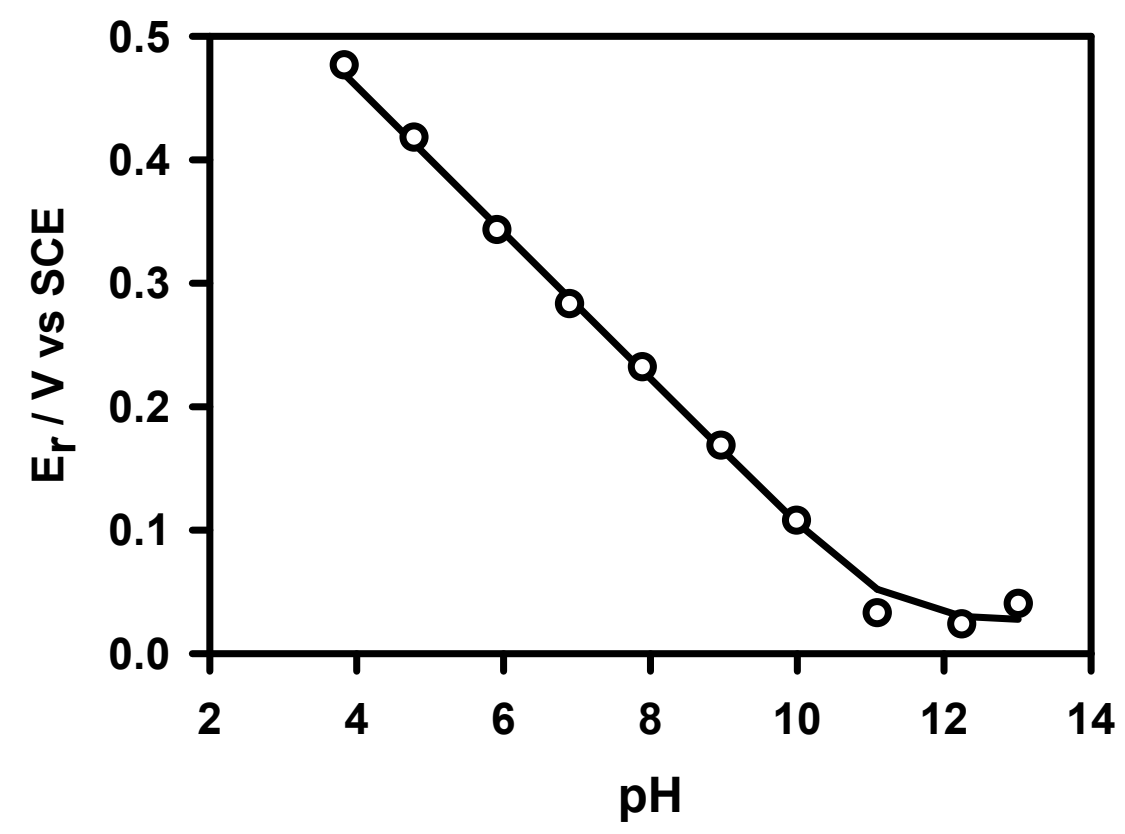

Figure 3.3. 2 Apparent Formal Potential vs $\mathrm{pH}^{32}$ 
be extracted accurately as an average of the anodic and cathodic peak positions. A plot of the apparent formal potential vs $\mathrm{pH}$ is seen in Figure 3.3.2. From $\mathrm{pH} 4-11, \mathrm{E}_{\mathrm{r}}$ shifts linearly with $\mathrm{pH}$ with a slope of $60 \mathrm{mV} / \mathrm{pH}$. $\mathrm{E}_{\mathrm{r}}$ becomes independent of $\mathrm{pH}$ at higher pHs. The line is a least squares fit of the data to the theoretical apparent formal potential from equation (1.11.2). The fit yields a $\mathrm{pK}_{\mathrm{a} 2}=11.3$ for the galvinol system with an $\mathrm{E}_{2}^{0^{\prime}}=+0.03 \mathrm{~V}$ vs SCE. Because the slope of the line stays Nernstian at the lowest $\mathrm{pHs}, \mathrm{pK}_{\mathrm{a} 1}$ (and thus $\mathrm{E}_{1}^{0^{\prime}}$ ) cannot be determined.

A closer examination of the thermodynamic behavior of the voltammogram reveals that at $\mathrm{pH}>11$, the peak shapes become less ideal with noticeable broadening of the waves. The FWHM goes from $100 \mathrm{mV}$ at $\mathrm{pH} 7$ to $150 \mathrm{mV}$ at $\mathrm{pH} 13$. This suggests that, at high $\mathrm{pH}$, the redox centers become thermodynamically heterogeneous, due to double layer effects and incorporation of the ionic species into the SAM.

It was impossible to collect data at $\mathrm{pH}<4$ because decomposition of the galvinol occurred at low pH (2-6) while in the oxidized (galvinoxyl) form. This became especially problematic because of the necessity for slower scan rates to assess the reversibility of the galvinol at lower $\mathrm{pHs}$. Figure $\mathbf{3 . 3 . 3}$ shows an initial voltammogram of galvinol at $\mathrm{pH} 7$, as well as a voltammogram of the same monolayer after multiple scans at slow scan rates $(\leq 0.1 \mathrm{~V} / \mathrm{s})$. The size of the original wave is reduced, while a new wave appears approximately $0.2 \mathrm{~V}$ negative of the original wave. Note that the FWHM of the new wave is less than that of the original wave (60 mV vs $100 \mathrm{mV})$. This 


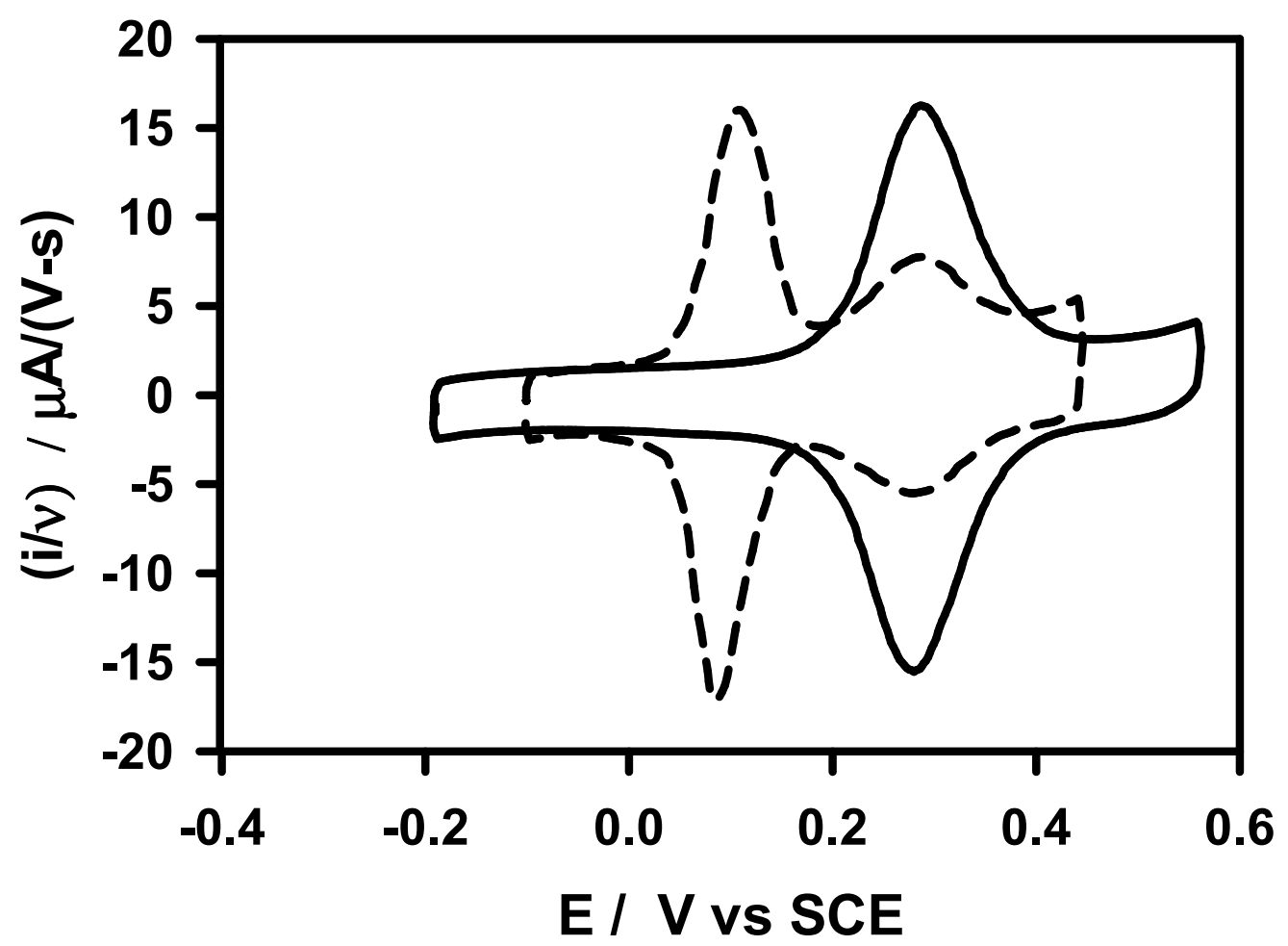

Figure 3.3. 3 Evidence of Galvinol Decomposition. Solid line is the monolayer before decomposition, and dashed line is the monolayer after decomposition. ${ }^{32}$ 
phenomenon suggests that a two electron process occurs with the new wave. In addition, the new wave exhibits the same dependence of formal potential on $\mathrm{pH}$ as galvinol. A Nersntian $\mathrm{pH}$ dependence is indicative of a system where the number of electrons equals the number of protons transferred. Thus, the smaller FWHM combined with Nernstian $\mathrm{pH}$ dependence suggests that the new wave behaves as a $2 \mathrm{e} 2 \mathrm{H}$ redox couple. It is possible that interaction with an adjacent galvinol could form a quinine/hydroquinone-like moiety, which whould exhibit $2 \mathrm{e} 2 \mathrm{H}$ behavior. The main consequence of this problem is that thermodynamic data could not be collected below $\mathrm{pH} 4$, and kinetic data (which requires many voltammograms) could not be collected below pH 7.

At $\mathrm{pH} \geq 7$, voltammograms were collected from a scan rate of $0.1 \mathrm{~V} / \mathrm{s}$ to 1000 V/s using 1, 2, 5 intervals through each decade. Figure 3.3.4 and Figure 3.3.5 illustrate the effect of scan rate on the shape of the voltammograms, at $\mathrm{pH} 9$ and $\mathrm{pH}$ 12. These two $\mathrm{pHs}$ were chosen because $\mathrm{pH} 9$ is below $\mathrm{pK}_{\mathrm{a} 2}$ and $\mathrm{pH} 12$ is above $\mathrm{pK}_{\mathrm{a} 2}$. As described in Chapter 1, the apparent transfer coefficient should be greater than 0.5 at $\mathrm{pK}_{\text {mid }}<\mathrm{pH}<\mathrm{pK}_{\mathrm{a} 2}$. Consequently, there should be a marked asymmetry in the voltammograms. According to theory, the anodic branch should be broader and should shift away from $E_{r}$ at a faster rate than the cathodic branch. The voltammograms in Figure 3.3.4 show this predicted asymmetry. At $\mathrm{pH}>\mathrm{pK}_{\mathrm{a} 2}$, theory predicts that the asymmetry disappears because $\alpha(0)$ becomes equal to 0.5 above $\mathrm{pK}_{\mathrm{a} 2}$. Figure 3.3 .5 is in agreement with the theory. CVs from Figure 3.3.5 are slightly distorted by iR drop, but clearly they are much more symmetrical than those in Figure 3.3.4. 


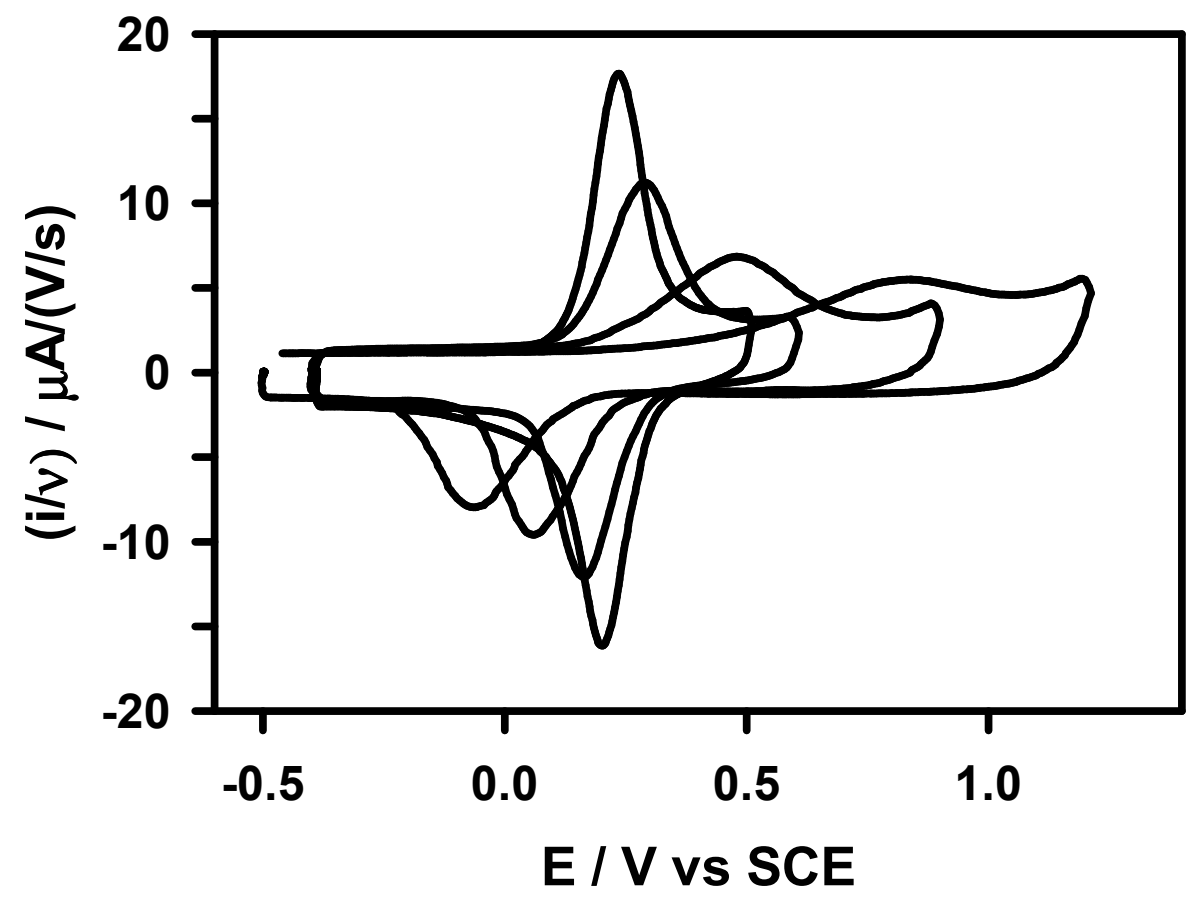

Figure 3.3. $4 \quad$ Voltammograms - multiple scan rates $-\mathrm{pH}$ 9. Scan rates are $0.1,10,100$, and $1000 \mathrm{~V} / \mathrm{s}$. Currents are normalized with respect to the scan rate. For this and all subsequent overlay voltammograms, the peak splitting increases progressively with scan rate. 


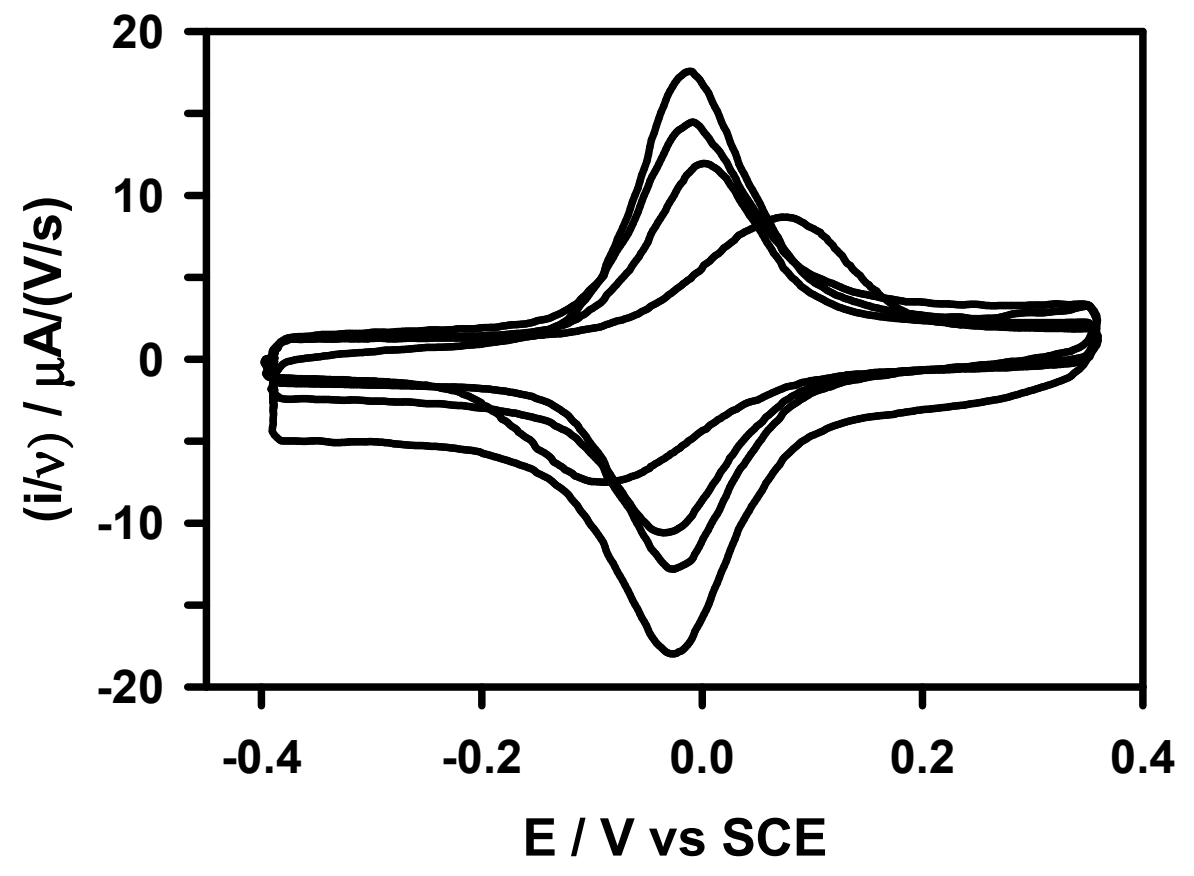

Figure 3.3. $5 \quad$ Voltammograms - multiple scan rates $-\mathrm{pH}$ 13. Scan rates are $0.1,10,100$, and $1000 \mathrm{~V} / \mathrm{s}$. Currents are normalized with respect to the scan rate. 
As discussed in the introduction, there are two methods for extracting kinetic data from voltammograms. In this preliminary study, kinetic information was measured at the reversible potential and not by Tafel analysis. For each $\mathrm{CV}$ of a given scan rate study, the apparent standard rate constant at zero overpotential was calculated for both the anodic wave and the cathodic wave; the two values were averaged to yield the apparent standard rate constant, $\mathrm{k}_{\mathrm{s}, \mathrm{app}}$. For CV's with peak splittings between 20 and $200 \mathrm{mV}, \mathrm{k}_{\mathrm{s}, \mathrm{app}}$ values were averaged together to yield an average apparent standard rate constant, $\mathrm{k}_{\mathrm{s}, \mathrm{app}, \text { avg. }}$. There were typically three or four scan rates of the proper peak splitting for each $\mathrm{pH}$. Kinetic results are seen in Figure 3.3.6. Using the $\mathrm{pK}_{\mathrm{a} 2}$ value of 11.3 from Figure 3.3.2, the least squares fit of the data from $\mathrm{pH} 10-13$ is shown as the nonlinear regression line. The fit yields a $k_{s, a p p, a v g}=4500 \mathrm{~s}^{-1}$. The fit assumes a potential dependent transfer coefficient with an arbitrary value for the reorganization energy of $0.6 \mathrm{eV}$. The fit appears to be quite good at high pH's. However, it appears that the data has a very steep slope at $\mathrm{pH}$ 9-10 and has a much shallower slope than the fit at low pH's. At low $\mathrm{pH}, \mathrm{k}_{\mathrm{s}, \mathrm{app}, \text { avg }}$ decreases at a rate less than the predicted order-of-magnitude per $2 \mathrm{pH}$ units.

\subsection{Results - Extensive Study}

After the publication of the preliminary results, the galvinol system was reexamined in much greater detail. Because of the issue of galvinol decomposition from the initial results, it was important to try and minimize the conversion of galvinol to 


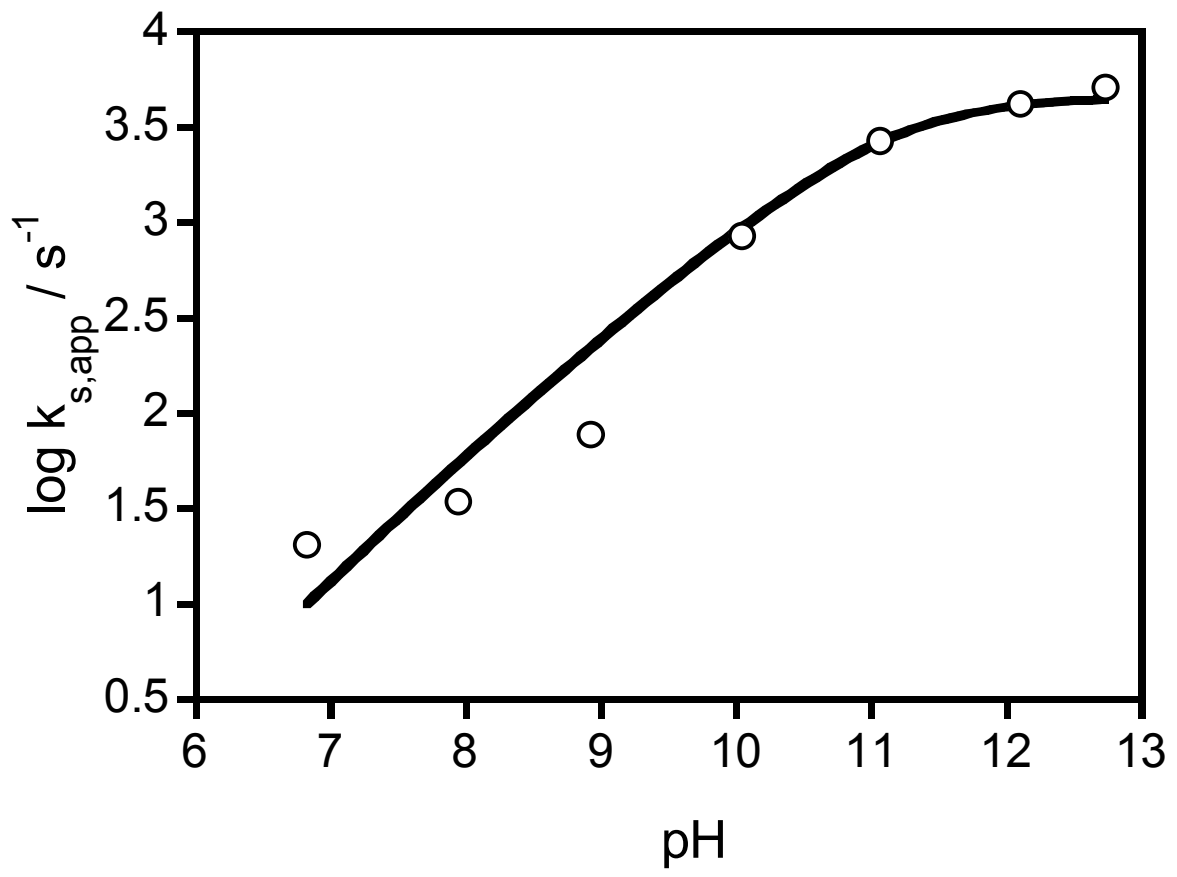

Figure 3.3. $6 \quad$ Average $\log \mathrm{k}_{\mathrm{s} \text {,app }} \mathrm{vs} \mathrm{pH}^{32}$ 
the quinone-hydroquinone-like complex. The same 2-compartment flow cell was used; however more effort was spent to keep the system as undisturbed as possible. This optimization was discussed in Chapter 2. The most important change centered around keeping dissolved oxygen out of the electrolyte. All electrolytes were purged continuously with argon prior to introduction into the flow cell reservoir. The flow of argon through the reservoir and over the working compartment was increased significantly. Extra holes were drilled in the working compartment lid to ensure that the argon flow would not disturb the surface level and pressurize the compartment. This care to ensure the absence of oxygen was successful in minimizing the formation of the decomposition product at all but the slowest scan rates $(\leq 0.02 \mathrm{~V} / \mathrm{s})$ at $\mathrm{pH}$ 's as low as 2 . At $\mathrm{pH}$ below 2 and at scan rates less than $0.02 \mathrm{~V} / \mathrm{s}$, the decomposition product caused too much interference to allow thermodynamic and kinetic analysis. The other important change involved flushing the cell during $\mathrm{pH}$ changes without turning off the cell. Turning off the cell was especially problematic at high $\mathrm{pH}$. The high potential fluctuations associated with switching the cell on or off caused loss of coverage of the attached galvinol. Leaving the cell turned on throughout analysis significantly improved monolayer stability, allowing the analysis of multiple $\mathrm{pH}$ 's with a single monolayer. It is worth noting that this flowcell optimization allowed the collection of data at scan rates an order of magnitude slower than in intial studies. Also, thermodynamic and kinetic data was collected from $\mathrm{pH} 2-14$, which is a significantly wider range compared to the preliminary study. 
Voltammograms and scan rate studies were collected using multiple monolayers and multiple electrodes over a pH range of 2-14. The electrolyte was composed of the $B-R$ buffer as described previously. Because of the decomposition problem described earlier, care was taken to keep the coverages smaller than before. Coverages of the redox couples on the electrodes averaged $1.0 \times 10^{-11} \mathrm{~mol} / \mathrm{cm}^{2}$. This had a desirable secondary effect of keeping iR drop insignificant at all but the highest scan rates ( $>500$ $\mathrm{V} / \mathrm{s})$.

A typical reversible voltammogram is seen in Figure 3.4.1. Small peak splittings $(>20 \mathrm{mV})$ combined with nearly ideal peak half-widths $(\sim 100 \mathrm{mV})$ suggest that the monolayer is well behaved with little thermodynamic heterogeneity. As seen before, at high pH's, the peak half-widths become noticeably larger. This peak broadening is attributed to increased double layer effects because of the large change in charge density for redox centers cycling between the 0 and -1 charge states ${ }^{37}$.

Figure 3.4.2 is a plot of the apparent formal potential vs $\mathrm{pH}$, which shows Nernstian $\mathrm{pH}$ dependence $(-60 \mathrm{mV} / \mathrm{pH})$ from $\mathrm{pH} 2-11$, with a break towards a $\mathrm{pH}$ independent value above $\mathrm{pH}$ 12. The solid line, which is a nonlinear least-squares fit of the data to the model, yields $\mathrm{pK}_{\mathrm{a} 2}=12.7$ and $\mathrm{E}_{2}{ }^{{ }^{\prime}}=-0.06 \mathrm{~V}$ vs SCE.

Both methods from the introduction are used in the extraction of the average apparent standard rate constant from the data. The Tafel data will be discussed later. The average $k_{s, a p p}$ data based on values at the apparent formal potential are summarized in Figure 3.4.3. Fitting the data to the model (the solid line) at $\mathrm{pH}$ greater than 7 (with $\mathrm{pK}_{\mathrm{a} 2}$ set at 12.7 ) yields $\mathrm{k}_{\mathrm{s} 2}=5600 \mathrm{~s}^{-1}$, independent of whether the transfer 


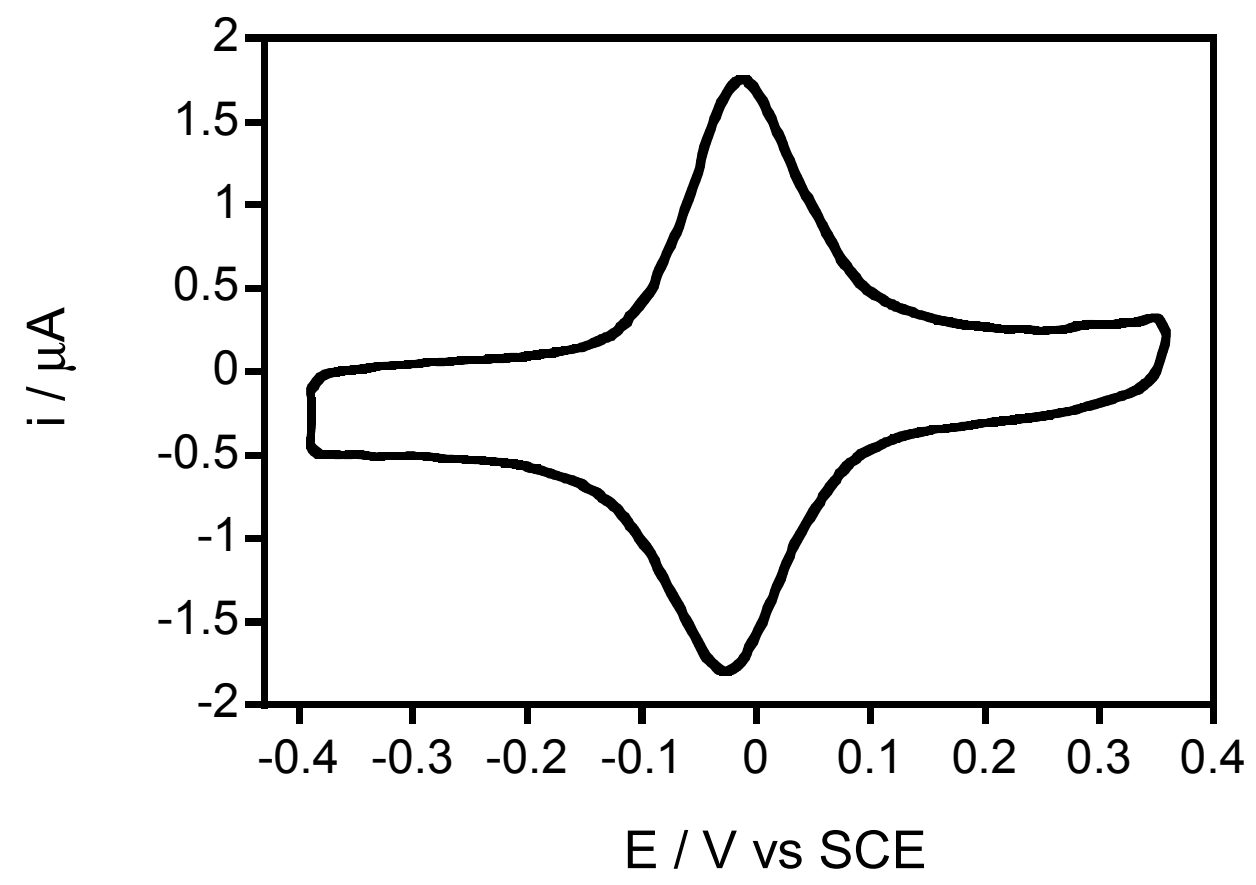

Figure 3.4. 1 Typical Reversible Voltammogram of Galvinol Monolayer. ${ }^{31}$ 


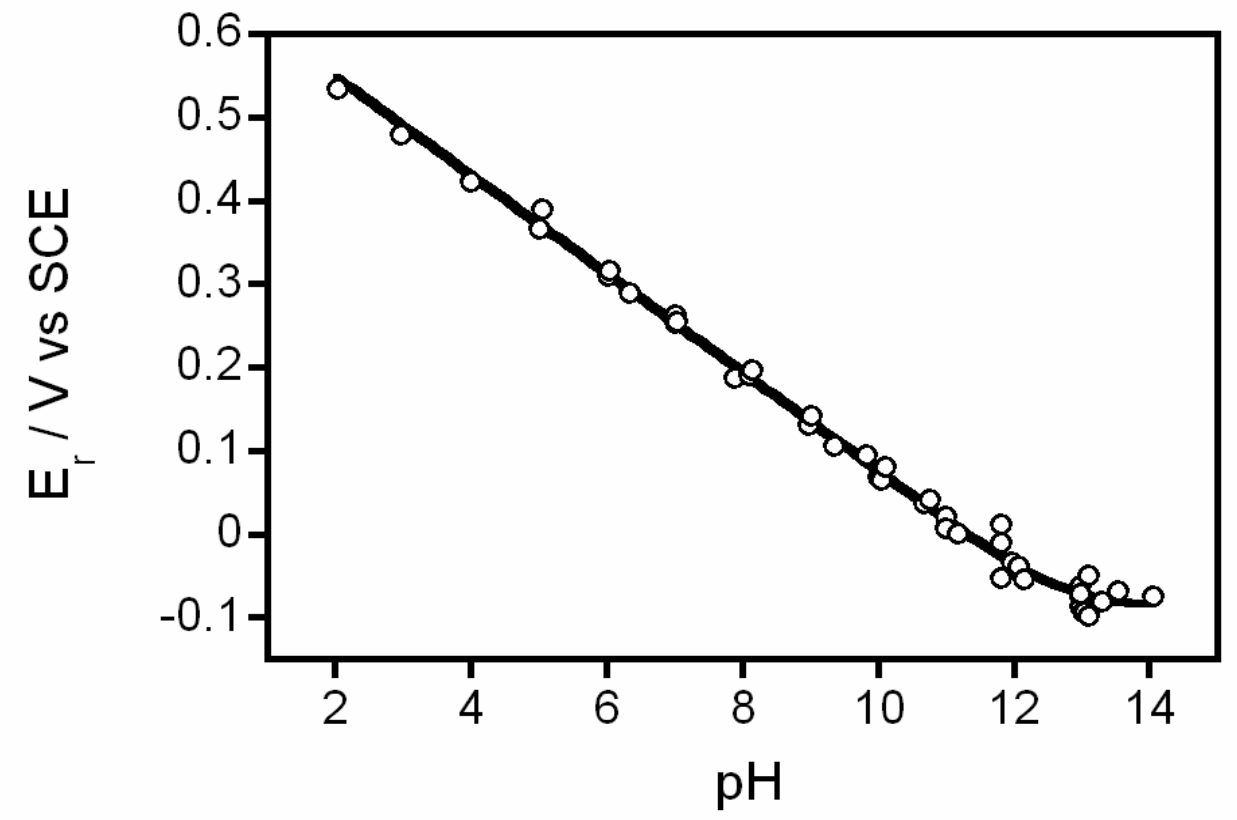

Figure 3.4. 2 Apparent Formal Potential vs $\mathrm{pH}^{31}$ 


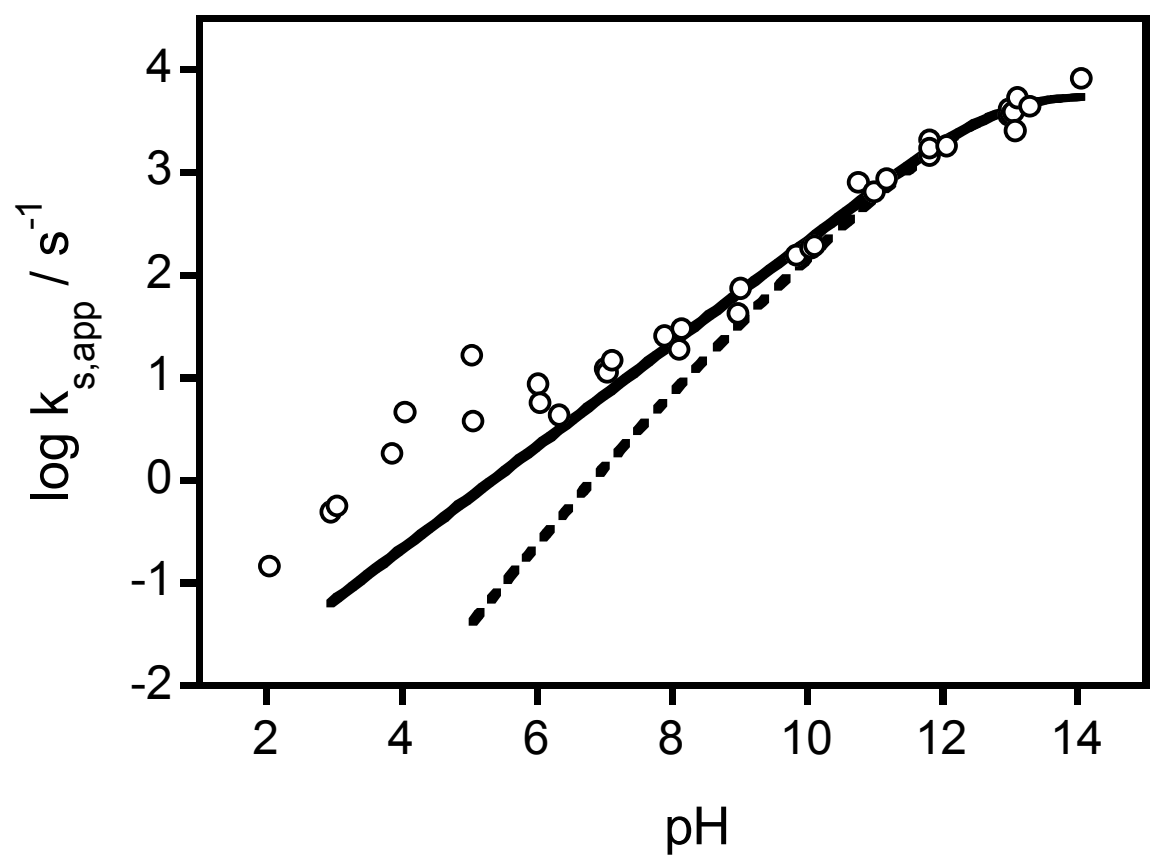

Figure 3.4. $3 \quad$ Average $\mathrm{k}_{\mathrm{s}, \mathrm{app}} \mathrm{vs} \mathrm{pH}-$ extracted at formal potential ${ }^{31}$ 


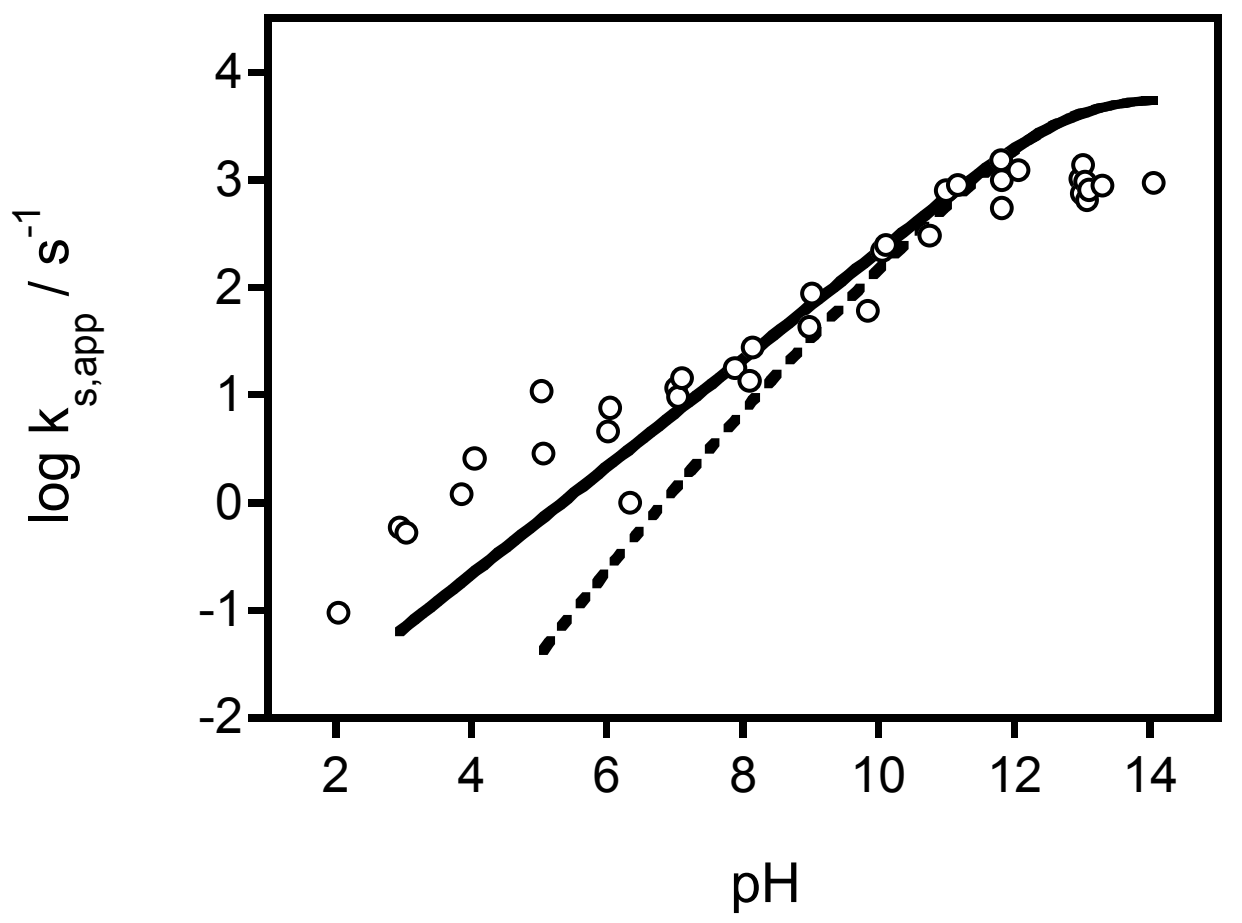

Figure 3.4. $4 \quad$ Average $\mathrm{k}_{\mathrm{s} \text {,app }} \mathrm{vs} \mathrm{pH}-$ extracted from Tafel Analysis ${ }^{31}$ 
coefficient is constant or potential dependent (for any reorganization energy, the theoretical plot of $\log \mathrm{k}_{\mathrm{s}} \mathrm{vs} \mathrm{pH}$ has a steeper slope than the plot for $\alpha=0.5$ ). The average $k_{\mathrm{s}, \mathrm{app}}$ data extrapolated from Tafel plots is summarized in Figure 3.4.4. Note that this plot is qualitatively similar to Figure 3.4 .3 , with the exception that the average $\mathrm{k}_{\mathrm{s}, \mathrm{app}}$ reaches a maximum of about $1100 \mathrm{~s}^{-1}$, significantly less than the other method. The fit in Figure 3.4.4 is identical to the fit from Figure 3.4.3, and is designed to illustrate the point that both data sets are nearly identical except at $\mathrm{pH}>10$. This discrepancy arises in part because the galvinols in the SAM's appear to be kinetically heterogeneous. The first method (measuring $\mathrm{k}_{\mathrm{s}}$ at the apparent formal potential) tends to emphasize kinetically faster galvinols because the data are obtained at a point where a small fraction of redox centers have been converted to product. The second method (extrapolation on a Tafel plot) emphasizes slower rate constants corresponding to $50 \%$ conversion of the reactant oxidation state to the product. Equally important, at high $\mathrm{pH}$, values of $\mathrm{k}_{\mathrm{s}}$ from Tafel plots are compromised by the difficulty of obtaining accurate rate constants when $\mathrm{k}_{\mathrm{s}}$ exceeds $1000 \mathrm{~s}^{-1}$ due to limitations in our equipment. The Model 173 universal programmer has a maximum scan rate of $1000 \mathrm{~V} / \mathrm{s}$. For the galvinol system, when $\mathrm{k}_{\mathrm{s}}$ exceeds $1000 \mathrm{~s}^{-1}$, there are not enough reliable points at overpotentials exceeding $0.1 \mathrm{~V}$ to accurately extract kinetic information. Extraction of kinetic data using data at insufficient overpotential systematically leads to low values, as seen in Figure 3.4.4. More reliable Tafel plots at high $\mathrm{pH}$ using existing equipment would require synthesizing the galvinol with a longer tether to slow down the kinetics, which is outside the scope of this project. A secondary reason that it appears the Tafel 
results are erroneous lies in the fact that a fit of the Tafel data, which allows both $\mathrm{pK}_{\mathrm{a} 2}$ and $\mathrm{k}_{\mathrm{s} 2}$ to float results in a $\mathrm{pK}_{\mathrm{a} 2}<10$, which clearly contradicts $\mathrm{pK}_{\mathrm{a} 2}=12.7$ from the thermodynamic data. A fit of the data from Figure 3.4.3, allowing both $\mathrm{pK}_{\mathrm{a} 2}$ and $\mathrm{k}_{\mathrm{s} 2}$ to float, results in a $\mathrm{pK}_{\mathrm{a} 2}=12.8$, which is identical to the thermodynamic data. For these reasons, it is felt that the data extracted at the apparent formal potential to be the more reliable data. It is important to note that both Figures 3.4.3 and 3.4.4 show a substantial deviation of the measured standard rate constants above the predicted standard rate constants of the model at pHs below 9. This deviation is discussed in

\section{Section 3.5 .}

In principle, Tafel analysis provides information on the reorganization energy of the redox species via the curvature of each branch. In all of the galvinol data, Tafel plots do not exhibit visible curvature over the somewhat limited accessable overpotential range (up to $0.4 \mathrm{~V}$ ). Consequently, the reorganization energy cannot be obtained from fitting the Tafel plots to the model. However, the lack of curvature does allow estimation of the apparent transfer coefficient using the Butler Volmer approach from Chapter 1. Slopes of the Tafel plots are obtained by linear regression of the data at overpotentials exceeding $0.1 \mathrm{~V}$. Figure 3.4.5 is a representative example of the results. The transfer coefficient can be calculated from the slope of each branch using the equations shown in Figure 1.2.1. The anodic transfer coefficient is typically higher than the cathodic transfer coefficient in each Tafel plot, as can be seen in Table 3.4.1. The transfer coefficient at the formal potential $\alpha(0)$ is obtained by averaging the anodic and cathodic values. A plot of $\alpha(0)$ vs $\mathrm{pH}$ is shown in Figure 3.4.6. The apparent 


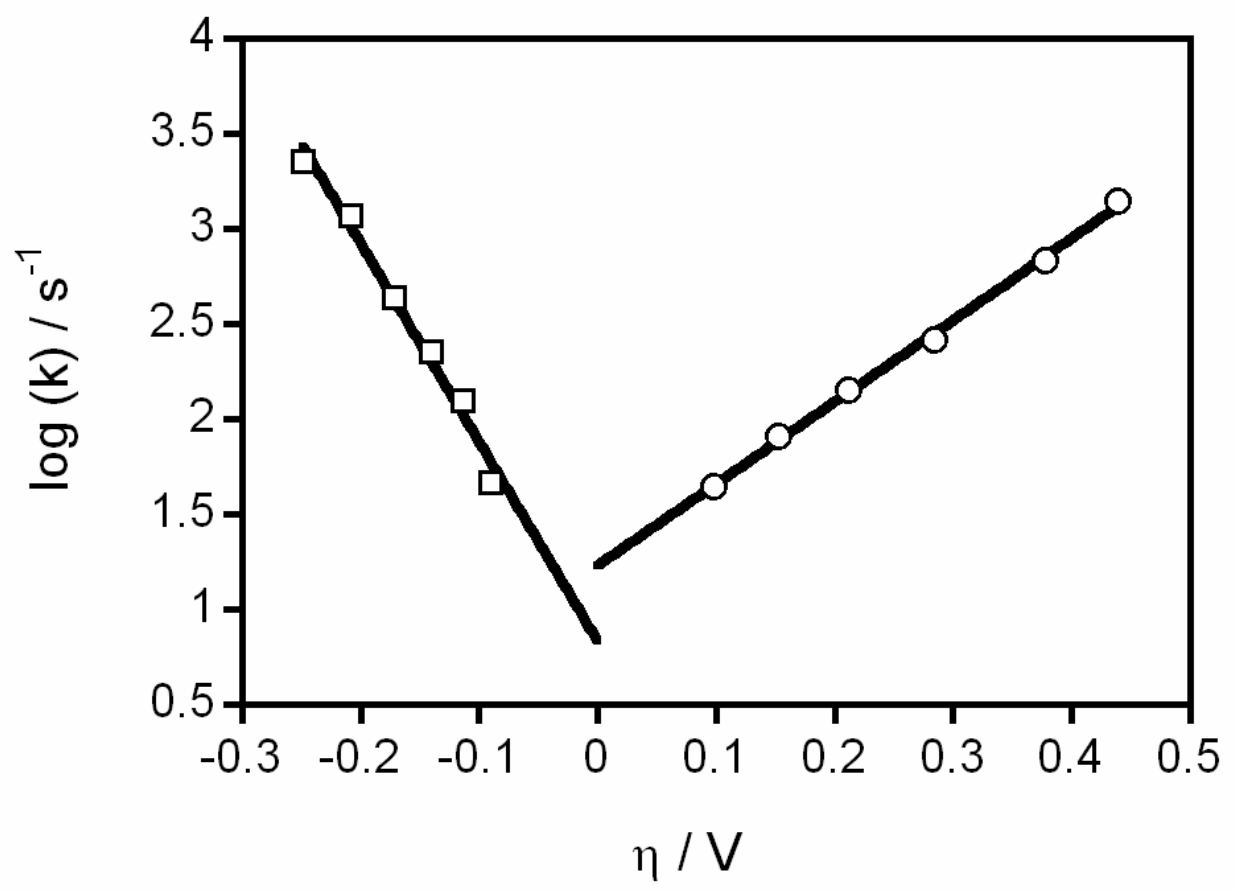

Figure 3.4. 5 Example Tafel Plot of Galvinol Monolayer at pH 8. ${ }^{31}$ 
Table 3.4. $1 \quad$ Apparent Transfer Coefficient vs pH

\begin{tabular}{|c|c|c|}
\hline$\overline{\mathrm{pH}}$ & Anodic $\alpha$ & Cathodic $\alpha$ \\
\hline 3.85 & 0.54 & 0.56 \\
\hline 4.04 & 0.57 & 0.51 \\
\hline 5.05 & 0.65 & 0.52 \\
\hline 5.05 & 0.68 & 0.53 \\
\hline 6.01 & 0.68 & 0.54 \\
\hline 6.06 & 0.71 & 0.60 \\
\hline 7.01 & 0.70 & 0.49 \\
\hline 7.04 & 0.70 & 0.65 \\
\hline 7.10 & 0.76 & 0.49 \\
\hline 7.88 & 0.64 & 0.43 \\
\hline 8.10 & 0.74 & 0.62 \\
\hline 8.14 & 0.73 & 0.52 \\
\hline 8.97 & 0.69 & 0.64 \\
\hline 9.01 & 0.81 & 0.49 \\
\hline 9.83 & 0.71 & 0.53 \\
\hline 10.05 & 0.74 & 0.52 \\
\hline 10.10 & 0.85 & 0.45 \\
\hline 10.99 & 0.86 & 0.41 \\
\hline 11.00 & 0.73 & 0.48 \\
\hline 11.80 & 0.58 & 0.51 \\
\hline 11.81 & 0.82 & 0.44 \\
\hline 12.06 & 0.72 & 0.48 \\
\hline 13.01 & 0.35 & 0.75 \\
\hline 13.07 & 0.58 & 0.56 \\
\hline
\end{tabular}




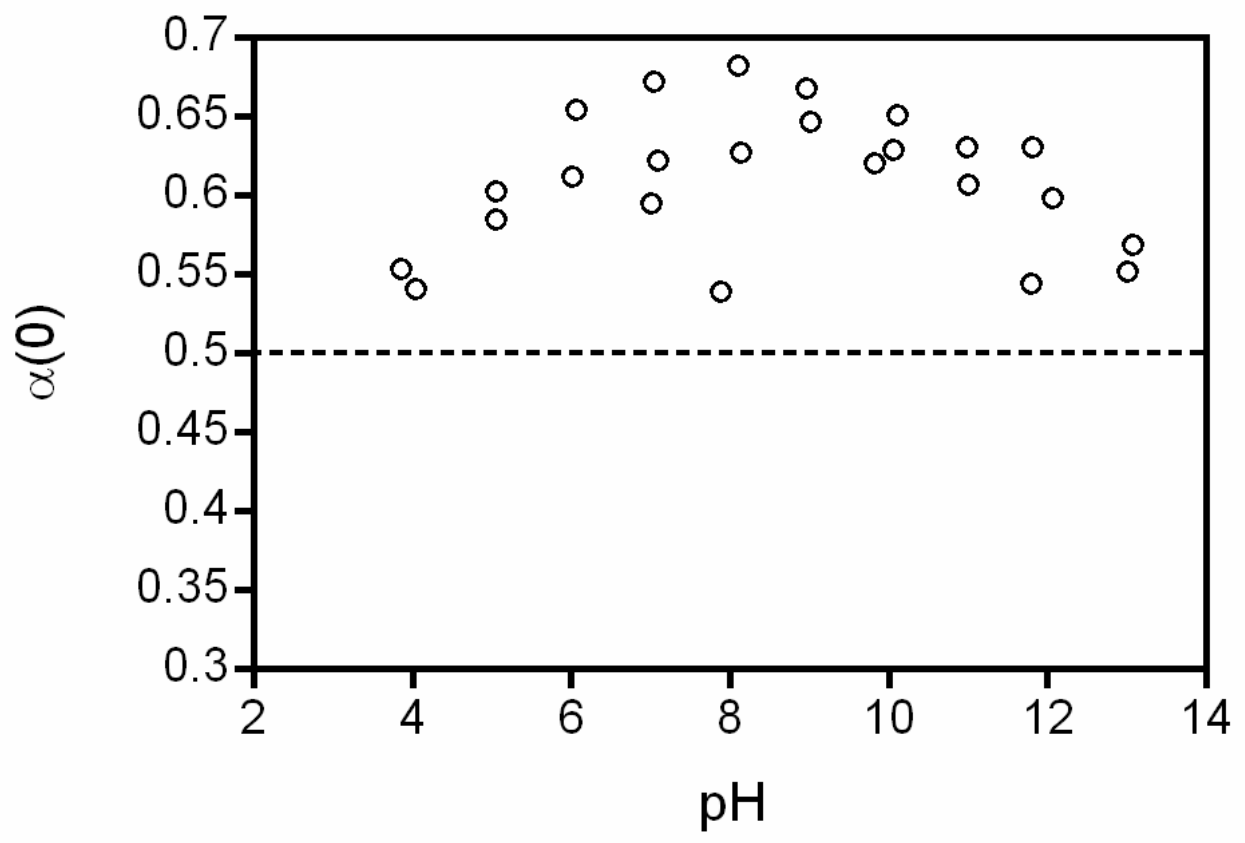

Figure 3.4. $6 \quad$ Average $\alpha(0) v \mathrm{pH}^{31}$ 
transfer coefficients are above 0.5 over the entire accessable $\mathrm{pH}$ range. When $\alpha(0)>$ 0.5 , kinetically controlled voltammograms exhibit anodic peaks which are broader and shifted farther from the formal potential compared to the cathodic peaks.

Voltammograms of galvinol show the predicted asymmetry at $2>\mathrm{pH}>11$. This asymmetry disappears at high $\mathrm{pH}$, as predicted by the model. The data is not quantitatively fitted to the model because of the deviation of the $\log k_{s}$ data from the model at low $\mathrm{pH}$.

\subsection{Discussion of Results}

Qualitatively, the analysis of both data sets gives similar results. Both systems appear to behave thermodynamically according to the model. The apparent formal potential exhibits the expected Nernstian slope at intermediate $\mathrm{pH}$ 's and approaches a value of $\mathrm{E}_{2}{ }^{{ }^{\prime}}$ where $\mathrm{pH}=\mathrm{pK}_{\mathrm{a} 2}$. The values for $\mathrm{E}_{2}{ }^{0^{\prime}}$ and $\mathrm{pK}_{\mathrm{a} 2}$ are somewhat different between the data sets. The first data set has an $\mathrm{E}_{2}{ }^{{ }^{\prime}}=+0.03 \mathrm{Vvs} \mathrm{SCE}$ and $\mathrm{pK}_{\mathrm{a} 2}=$ 11.3, while the second data set reports an $\mathrm{E}_{2}{ }^{{ }^{\prime}}=-0.06 \mathrm{~V}$ vs SCE and $\mathrm{pK}_{\mathrm{a} 2}=12.7$. The cause for these differences is not known. It is possible that the galvinol $\mathrm{pK}_{\mathrm{a} 2}$ is sensitive to buffer composition. Kinetically, again the results are qualitatively similar but quantitatively different, with the first data set yielding a $\mathrm{k}_{\mathrm{s} 2}=4600 \mathrm{~s}^{-1}$, and the second data set yielding a $\mathrm{k}_{\mathrm{s} 2}=5600 \mathrm{~s}^{-1}$. We believe the values for the second data set to be more reliable because more data points were used to fit the data to the model. In all other aspects, the results of both data sets give similar results. Thus, the 
remainder of this discussion will refer to both data sets indiscriminately as one collective data set, unless specified differently in the text.

As stated earlier, thermodynamically, the galvinol system exhibits the expected behavior of a $1 \mathrm{e} 1 \mathrm{H}$ system. There is increased scatter in the data at high pH's. This can be attributed somewhat to the presence of alkaline error in the $\mathrm{pH}$ electrode, and to the fact that several different electrolytes were used to explore the high $\mathrm{pH}$ behavior. Thermodynamic heterogeneity, which was noted as peak broadening at the highest pH's might also contribute to slight discrepancies in the formal potential, especially if the contribution of double layer effects differed between monolayers. Variations in liquid junction potentials at the reference electrode tip could also account for increased scatter in the apparent formal potential.

The value of $\mathrm{pK}_{\mathrm{a} 2}(12.7)$ is somewhat consistent with the phenol-like structure of galvinol. While the increased resonance effects of galvinol would be expected to lower the $\mathrm{pK}_{\mathrm{a} 2}$ relative to the $\mathrm{pK}_{\mathrm{a}}$ of phenol (9.99), they may be offset by the electron donating effects of the multiple tert-butyl substituents (the $\mathrm{pK}_{\mathrm{a}}$ of 2,4-di-tert-butylphenol is 11.57 ) and possibly by a lower dielectric constant if the galvinol partially penetrates the $\mathrm{SAM}^{53}$. A much lower value is anticipated for $\mathrm{pK}_{\mathrm{a} 1}$ because protonation of the electron-deficient galvinoxyl is expected to be energetically unfavorable. This can be seen in the hydroquinone system, whose $\mathrm{pK}_{\mathrm{a}}$ is 9.85 , and the one-electron oxidation product $\mathrm{QH}_{2}{ }^{+}$, whose $\mathrm{pK}_{\mathrm{a}}$ is estimated to be $-1 \cdot 1^{54}$. The electron transfer kinetic behavior (see below) of galvinol suggests that $\mathrm{pK}_{\mathrm{a} 1}$ could be quite negative. 
Asymmetry in the CV's at intermediate $\mathrm{pH}$ is predicted when the transfer coefficients are potential dependent and $\alpha(0) \neq 0.5$, and not when the transfer coefficient is equal to 0.5 at all overpotentials. The voltammograms in Figure 3.3.4. clearly show asymmetry between the anodic and cathodic peaks. This asymmetry is consistent with predictions of a redox couple at a $\mathrm{pH}$ between $\mathrm{pK}_{\text {mid }}$ and $\mathrm{pK}_{\mathrm{a} 2}$, according to the model. As seen in Figure 3.3.5, the asymmetry disappears at high pH, where $\mathrm{pH}>\mathrm{pK}_{\mathrm{a} 2}$, representative of the fact that $\alpha(0)=0.5$. To our knowledge, this result is the first evidence showing that the apparent transfer coefficient for a coupled electron/proton transfer process is dependent on potential in the manner predicted by the Marcus DOS theory.

Kinetically, the galvinol system appears to follow the model at pH greater than 7 . The nonlinear fit from both data sets (Figures 3.3.6 and 3.4.3) show good agreement with the theory. Over this $\mathrm{pH}$ range near $\mathrm{pK}_{\mathrm{a} 2}$, the fit is insensitive to the reorganization energy, so the fit should yield an accurate $\mathrm{k}_{\mathrm{s} 2}$, independent of $\lambda$. However, this also means that it is impossible to estimate the reorganization energy. $\lambda$ would more accurately be calculated using curvature in Tafel plots. Unfortunately, limitations in our equipment will not allow the collection of data of sufficient overpotentials to estimate the curvature of the Tafel plots. Thus, the reorganization energy is still unknown. It would be necessary to synthesize a galvinol SAM with a longer alkane chain length in order to bring $\mathrm{k}_{\mathrm{s} 2}$ into a more accessible range.

At lower pH's, the data clearly deviate from the model. The rate of decrease in $\mathrm{k}_{\mathrm{s}, \mathrm{app}}$ as a function of $\mathrm{pH}$ is much less than that predicted by theory. The cause for the 
poor fit of $\mathrm{k}_{\mathrm{s}, \text { app }}$ to theory at lower $\mathrm{pH}$ 's is not known. Voltammogram distortion due to iR drop is only significant at the highest pH's, where voltammograms were collected at greater than $500 \mathrm{~V} / \mathrm{s}$. Double layer effects (partitioning of the galvinol into the SAM) is possible, which could cause kinetic deviation. However, this is unlikely because of the ideal nature of the reversible voltammograms at low $\mathrm{pH}$. Only at high $\mathrm{pH}$ do the waves broaden out, suggesting double layer effects. Another potential cause lies in the different buffer compositions and ionic strengths used in the initial study. We had observed that adding $1.0 \mathrm{M} \mathrm{Na}_{2} \mathrm{SO}_{4}$ to $\mathrm{pH} 9$ borate buffer caused $\mathrm{k}_{\mathrm{s} \text {,app }}$ to increase by a factor of 2. The possibility of electrolyte effects was addressed in the second data set, where an electrolyte buffer of a single composition was used. $0.5 \mathrm{M} \mathrm{K}_{2} \mathrm{SO}_{4}$ was added to buffer the ionic strength. Thus, electrolyte effects should have been minimized. However, the deviation of $\mathrm{k}_{\mathrm{s}, \mathrm{app}}$ at low $\mathrm{pH}$ persists in the second data set. A more thorough discussion of this deviation will be provided later.

Despite the clear deviation of log $\left(\mathrm{k}_{\mathrm{s}}\right)$ from the model, a number of interesting observations can be made about the galvinol system. First, the average $\mathrm{k}_{\mathrm{s} \text {,app }}$ continues to decrease down to $\mathrm{pH} 2$. If one assumes that $\mathrm{ks} 1$ is roughly equal to $\mathrm{k}_{\mathrm{s} 2}$, then $\mathrm{pK}_{\text {mid }}$ can be no higher than 2. Thus by extrapolation of the $\log \mathrm{k}_{\mathrm{s} \text {,app }} \mathrm{vs} \mathrm{pH}$ plot would result in a $\mathrm{pK}_{\mathrm{a} 1}$ of no higher than -8.7 , which is quite low. Thus, it can be concluded that over the explored $\mathrm{pH}$ range, the protonated galvinoxyl does not participate significantly in the electron transfer process. The relevant kinetic scheme is galvinoxyl - deprotonated galvinol - galvinol (O - P - R). 
Table 3.5. 1

Centers $^{31}$

Standard Rate Constants vs chain lengths for two redox

\begin{tabular}{||c|c|c|c|c|c||}
\hline Redox Center & \# $\mathbf{C H}_{\mathbf{2}}$ & Link & \#atoms $^{\mathbf{a}}$ & $\mathbf{k}_{\mathbf{s}}^{\mathbf{b}} / \mathbf{s}^{\mathbf{- 1}}$ & Ref. \\
\hline \hline $\mathbf{R u}\left(\mathbf{N H}_{\mathbf{3}}\right)_{\mathbf{5}}(\mathbf{p y})$ & 15 & $-\mathrm{C}(\mathrm{O}) \mathrm{N}(\mathrm{H}) \mathrm{CH}_{2}-$ & 19 & 1.2 & 55 \\
\hline & 11 & & 15 & 80 & 56 \\
\hline & 7 & & 11 & 5000 & 56 \\
\hline Ferrocene & 15 & $-\mathrm{N}(\mathrm{H}) \mathrm{C}(\mathrm{O})-$ & 18 & 6 & 57 \\
\hline & 12 & & 15 & 180 & \\
\hline & 8 & & 11 & 17000 & \\
\hline Ferrocene & 15 & $-\mathrm{OC}(\mathrm{O})-$ & 18 & 5 & 58 \\
\hline & 12 & & 15 & 210 & \\
\hline & 8 & & 11 & 33000 & \\
\hline
\end{tabular}

ancludes sulfur atom. ${ }^{b}$ Based on linear regression of $\log \left(\mathrm{k}_{\mathrm{s}}\right) \mathrm{vs} \# \mathrm{CH}_{2}$ 's in the cited reference. 
It is of interest to compare $\mathrm{k}_{\mathrm{s} 2}$ of the galvinol system to standard rate constants of other systems attached to SAM's. Extensive data indicate that, in the absence of extended conjugation, a primary determinate of the standard rate constant is the number of atoms connecting the redox center to the electrode. Linear regression of $\log \left(k_{s}\right)$ vs \# of methylenes allow prediction of $\log \left(k_{s}\right)$ for a given number of atoms, and can be seen in Table 3.5.1. The galvinol redox center is normally just the two phenyl rings substituted with the t-butyl groups and the connecting carbon. If that is the case, then 15 atoms connect the galvinol with the electrode. As can be seen in Table 3.5.1, a 15 atom linkage results in a standard rate constant of $80-210 \mathrm{~s}^{-1}$ for the various redox centers, which is considerably less than the $\mathrm{k}_{\mathrm{s} 2}$ of galvinol. If the comparison is valid, the higher standard rate constant for galvinol implies that it has a small reorganization energy, compared to the other redox centers. Reorganization energies of the Ru complex and ferrocene are approximately $0.8 \mathrm{eV}$. The idea that the reorganization energy for galvinol is much smaller than $0.8 \mathrm{eV}$ is unlikely considering the lack of curvature in the Tafel plots at overpotentials up to $0.4 \mathrm{~V}$. It seems reasonable that the phenyl ring attached to the galvinol is also part of the redox center (i.e., the electronic coupling of the phenyl ring to the galvinol moiety is strong, which is likely due to resonance with the other two phenyl rings). If this is the case, then only 11 atoms connect the Ru complexes and the two ferrocenes. Values of $k_{s}$ for 11 atoms are 5000$33000 \mathrm{~s}^{-1}$. Because the $\mathrm{k}_{\mathrm{s} 2}$ for galvinol is comparable to the standard rate constant for the Ru complex, the reorganization energy should be similar. 


\section{Chapter 4 Proton Coupled Electron Transfer Kinetic Studies of Polypyridyl Osmium Complexes}

\subsection{Introduction - The Osmium System}

Numerous organometallic compounds with different central metal atoms have been reported to exhibit PCET ${ }^{14 ; 59-61}$. Metal-aqua complexes can undergo PCET through the two hydrogens on each water group. Typically, noncomplexed metals can form a number of oxide/aqua based complexes depending on the charge of the metal ${ }^{14}$. Unfortunately, this results in complex multi-proton/electron transfers. It is common to simplify the metallic system by complexing the inner-coordination sphere with non-ionizable ligands. There are many reported metals used ${ }^{62-64}$, but by far the most common systems use Ruthenium and Osmium. Polypyridyl ligands are commonly used because of their strong interaction with the metal. Meyer has developed the use of $\left[\mathrm{M}(\mathrm{bpy})_{2}\left(\mathrm{OH}_{2}\right)_{2}\right]^{2+}(\mathrm{M}=\mathrm{Ru}, \mathrm{Os})^{65}$. This system is interesting because it has only four ionizible hydrogens, and under the proper conditions can be separated into four distinct $1 \mathrm{e} 1 \mathrm{H}$ reactions. Unfortunately, this is still rather complex. Meyer has also developed a complex with only one aqua site, $\left[\mathrm{M}(\mathrm{tpy})(\mathrm{bpy}) \mathrm{OH}_{2}\right]^{2+}(\mathrm{M}=$ $\mathrm{Ru}, \mathrm{Os})^{66}$. These compounds even further limit the number of potential PCET sites. In the Ru system, two distinct $1 \mathrm{e} 1 \mathrm{H}$ reactions occur at very similar potentials, making the discrete analysis of each process nearly impossible. However, in $\left[\mathrm{Os}(\mathrm{tpy})(\mathrm{bpy}) \mathrm{OH}_{2}\right]^{\mathrm{nt}}$, the two $1 \mathrm{e} 1 \mathrm{H}$ processes are separated by a potential of $>0.2 \mathrm{~V}$ at most $\mathrm{pH}$ 's. 
Figure 4.1.1 is a Pourbaix diagram of the $\left[\mathrm{Os}(\mathrm{tpy})(\mathrm{bpy}) \mathrm{OH}_{2}\right]^{\mathrm{nt}}$ system. Clearly the $\mathrm{Os}^{2+/ 3+}$ appears to be a good choice for study. Both $\mathrm{pK}_{\mathrm{a} 1}$ and $\mathrm{pK}_{\mathrm{a} 2}$ lie within a $\mathrm{pH}$ range accessible in aqueous solution ( 2 and 8 , respectively). $\mathrm{E}_{1}{ }^{0}$ and $\mathrm{E}_{2}{ }^{0}$ are well within the potential window of a gold electrode $(+0.36 \mathrm{~V}$ vs. SCE and $0 \mathrm{~V}$ vs. SCE, respectively). Also, the $3+/ 4+$ wave starts merging with the $2+/ 3+$ wave at a $\mathrm{pH}$ greater than $\mathrm{pK}_{\mathrm{a} 2}$. Thus, thermodynamic and kinetic data could be extracted in all three dynamic areas: low, intermediate, and high $\mathrm{pH}$.

While the $\left[\mathrm{Os}(\mathrm{tpy})(\mathrm{bpy}) \mathrm{OH}_{2}\right]^{2+/ 3+}$ appears to be a good system to explore, it is not possible to get kinetic information from the complex in free solution because of the high rates of electron transfer associated with free solution kinetics. As discussed earlier, the attachment of redox couples to a monolayer is a convenient means of slowing the kinetics into a measureable range. In order to attach the osmium complex to a monolayer, the molecule must be modified to include a tether. A number of scenarios are used today to attach an organometallic complex to a monolayer, using a

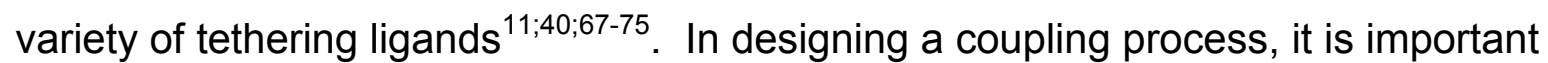
minimize changes to the molecule. Specifically, in the case of the osmium complex, it is important to provide a place for attachment without changing the electrostatic environment of the original complex. Changing the structure of the complex can have significant effects on the thermodynamics and kinetics. The osmium complex is a polypyridyl system with one coordinating water group. It has been shown that polypyridyl osmium complexes have similar thermodynamics regardless of the nature of the bonding between polypyridines. Table 4.1.1 includes published thermodynamic 


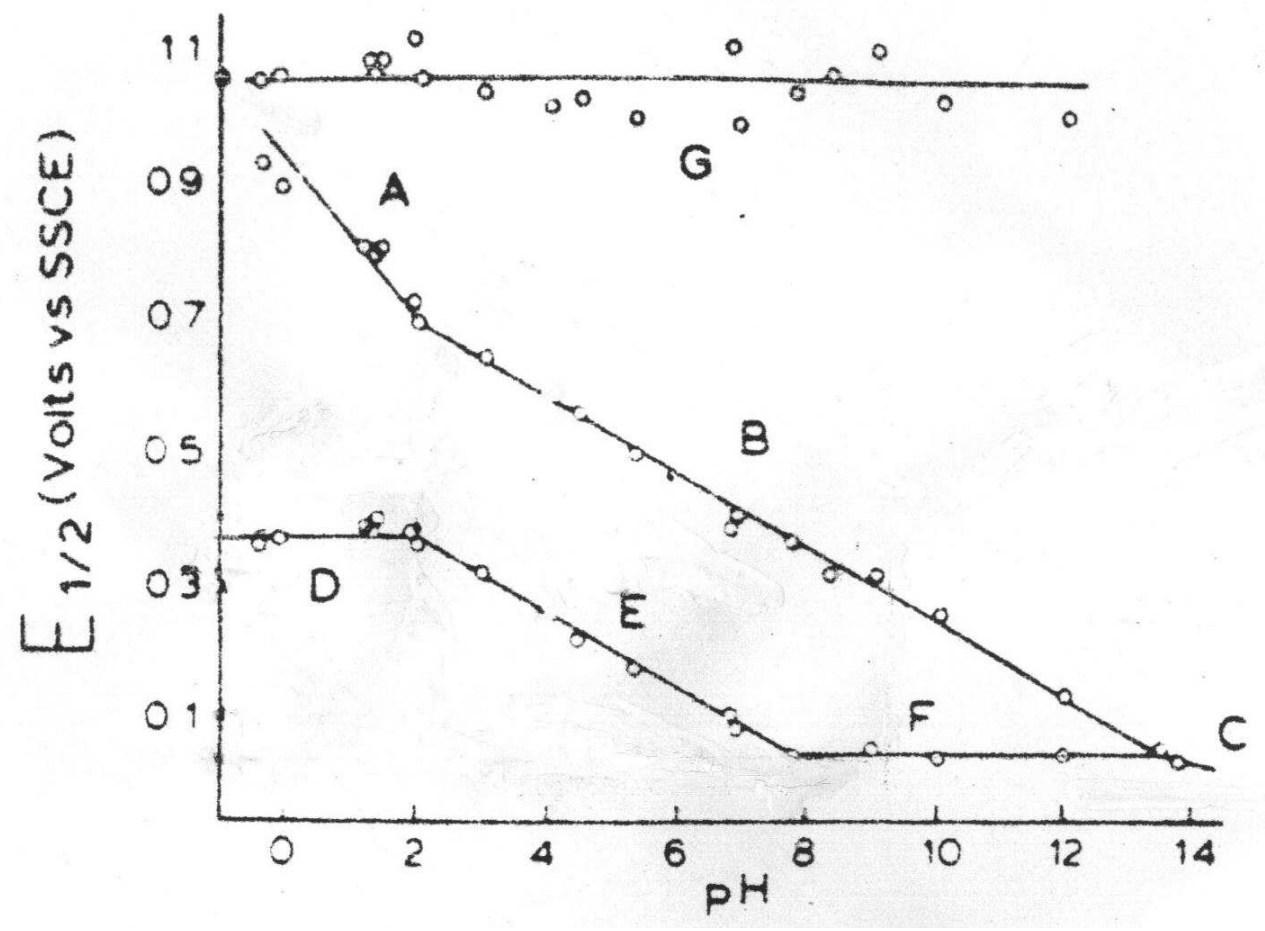

Figure 4.1. 1 Pourbaix Diagram of $\left[\mathrm{Os}(\mathrm{tpy})(\mathrm{bpy}) \mathrm{OH}_{2}\right]^{\mathrm{n}+66}$. Data collected in $0.1 \mathrm{M}$ aqueous buffer using a glassy-carbon working electrode. (A) $\mathrm{Os}^{\mathrm{iv}}=\mathrm{O}^{2+} / \mathrm{Os}^{\mathrm{iii}}-$ $\mathrm{OH}_{2}{ }^{3+}$; (B) $\mathrm{Os}^{\mathrm{iv}}=\mathrm{O}^{2+} / \mathrm{Os}^{\mathrm{iii}}-\mathrm{OH}^{2+}$; (C) Os $s^{\mathrm{iv}}=\mathrm{O}^{2+} / \mathrm{Os}^{\mathrm{ii}}-\mathrm{OH}^{+}$; (D) $\mathrm{Os}^{\mathrm{iii}}-\mathrm{OH}_{2}{ }^{3+} / \mathrm{Os}^{\mathrm{ii}}-\mathrm{OH}_{2}{ }^{2+}$; (E) $\mathrm{Os}^{\mathrm{iii}}-\mathrm{OH}^{2+} / \mathrm{Os}^{\mathrm{ii}}-\mathrm{OH}_{2}{ }^{2+}$; (F) $\mathrm{Os}^{\mathrm{iii}}-\mathrm{OH}^{2+} / \mathrm{Os}^{\mathrm{ii}}-\mathrm{OH}^{+}$; (G) Os $\mathrm{Os}^{\mathrm{v}}=\mathrm{O}^{3+} / \mathrm{Os}^{\mathrm{iv}}-\mathrm{O}^{2+}$ 
Table 4.1. 1 Formal Potential of $\left[\mathrm{Os}\left(\mathrm{L}_{5}\right) \mathrm{Cl}\right]^{+}$attached to a gold electrode.

Data collected using various aqueous electrolytes.

\begin{tabular}{|c|c|c||}
\hline COMPOUND & $\begin{array}{r}\text { FORMAL } \\
\text { POTENTIAL }(V) \text { VS } \\
\text { SCE) }\end{array}$ & REFERENCE \\
\hline$\left[\mathrm{Os}(\mathrm{bpy})_{2}(\mathrm{dipy}) \mathrm{Cl}\right]^{+}$ & .26 & 67 \\
\hline$\left[\mathrm{Os}(\mathrm{bpy})_{2}(\mathrm{dipy}) \mathrm{Cl}\right]^{+}$ & .31 & 40 \\
\hline$\left[\mathrm{Os}(\mathrm{bpy})_{2}(\mathrm{vpy}) \mathrm{Cl}\right]^{+}$ & .36 & 76 \\
\hline$\left[\mathrm{Os}(\mathrm{bpy})_{2}(\mathrm{py}=\mathrm{py}) \mathrm{Cl}\right]^{+}$ & .33 & 76 \\
\hline$\left[\mathrm{Os}(\mathrm{bpy})_{2}(\mathrm{bpt}) \mathrm{Cl}\right]^{+}$ & .26 & 77 \\
\hline$\left[\mathrm{Os}(\mathrm{bpy})_{2}(\mathrm{py}) \mathrm{Cl}\right]^{+}$ & .31 & 78 \\
\hline$\left[\mathrm{Os}(\mathrm{bpy})_{2}(\mathrm{pypy}) \mathrm{Cl}\right]^{+}$ & .28 & 68 \\
\hline$\left[\mathrm{Os}(\mathrm{bpy})_{2}(\mathrm{py}-\mathrm{SH}) \mathrm{Cl}\right]^{+}$ & .15 & 78 \\
\hline \hline
\end{tabular}


data on $\left[\mathrm{Os}(\mathrm{L})_{5} \mathrm{Cl}\right]^{+}$complexes attached to a gold electrode, where $\mathrm{L}$ represents a pyridine-like ligand . Figure 4.1.2 represents the structures of the various polypyridyl ligands. In this research project, we were interested in synthesizing an osmium polypyridyl aqua complex with an amino-terminated or alcohol-terminated tether. An amino- or alcohol-terminus is necessary to couple the redox molecule to the monolayer using the coupling process. Details on this process will be provided in a later section. Any tether would have to be attached to one of the pyridyl complexes: pyridine, bipyridine, or terpyridine. An amino-modified pyridine ligand is available commercially, 4-aminomethyl pyridine. This ligand has been successfully used as a tether for a number of redox couples ${ }^{70 ; 71 ; 74}$. While there were no commercially available aminomodified bipyridine or terpyridine ligands, an alcohol-modified terpyridine ligand, tpy$\mathrm{OH}$, was donated to us by Prof. Debbie Mohler. The structure of tpy-OH is seen in Figure 4.1.3. Using the two available tether ligands, research focused on making two osmium aqua complexes - $\left[\mathrm{Os}(\mathrm{tpy}-\mathrm{o}-)(\mathrm{bpy}) \mathrm{OH}_{2}\right]^{2+/ 3+}$ and $\left[\mathrm{Os}(\mathrm{bpy})_{2}(4-\mathrm{AMP}) \mathrm{OH}_{2}\right]^{2+/ 3+}$. In making these "tethered" molecules, it is assumed that the tether should have little effect on the thermodynamics and the reorganization energy of the system.

The synthesis of the two target compounds can theoretically be achieved via a number of different pathways. In our research, the synthesis of [Os(tpy-o)(bpy) $\left.\mathrm{OH}_{2}\right]^{2+/ 3+}$ was attempted using one reaction scheme, and the synthesis of $\left[\mathrm{Os}(\mathrm{bpy})_{2}(4-\mathrm{AMP}) \mathrm{OH}_{2}\right]^{2+/ 3+}$ was attempted using three reaction schemes. Synthesis of these molecules was achieved with varying success using the different pathways, and will be discussed in detail in the following sections. 


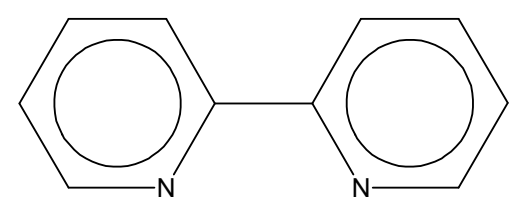

2,2'-bipyridine (bpy)

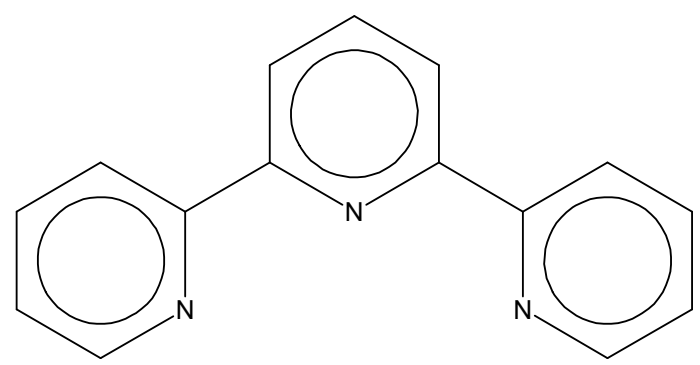

$2,2^{\prime}: 6^{\prime}, 2^{\prime \prime}$ - terpyridine (tpy)

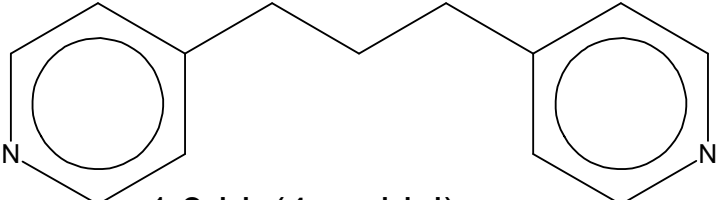

1,3-bis(4-pyridyl)propane (dipy)

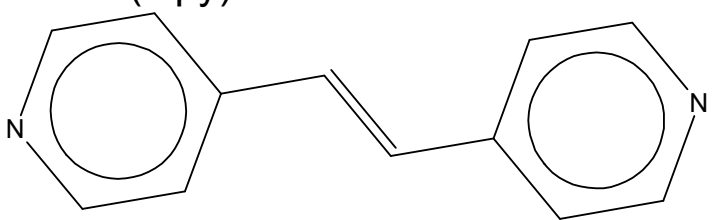

trans-1,2-bis(4-pyridyl)ethylene (py=py)

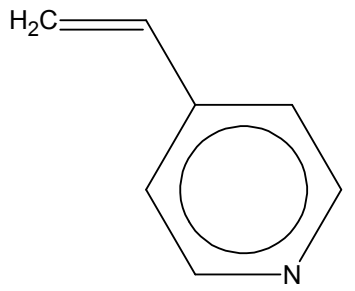

4-vinylpyridine (vpy)

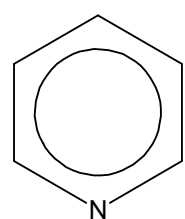

pyridine (py)
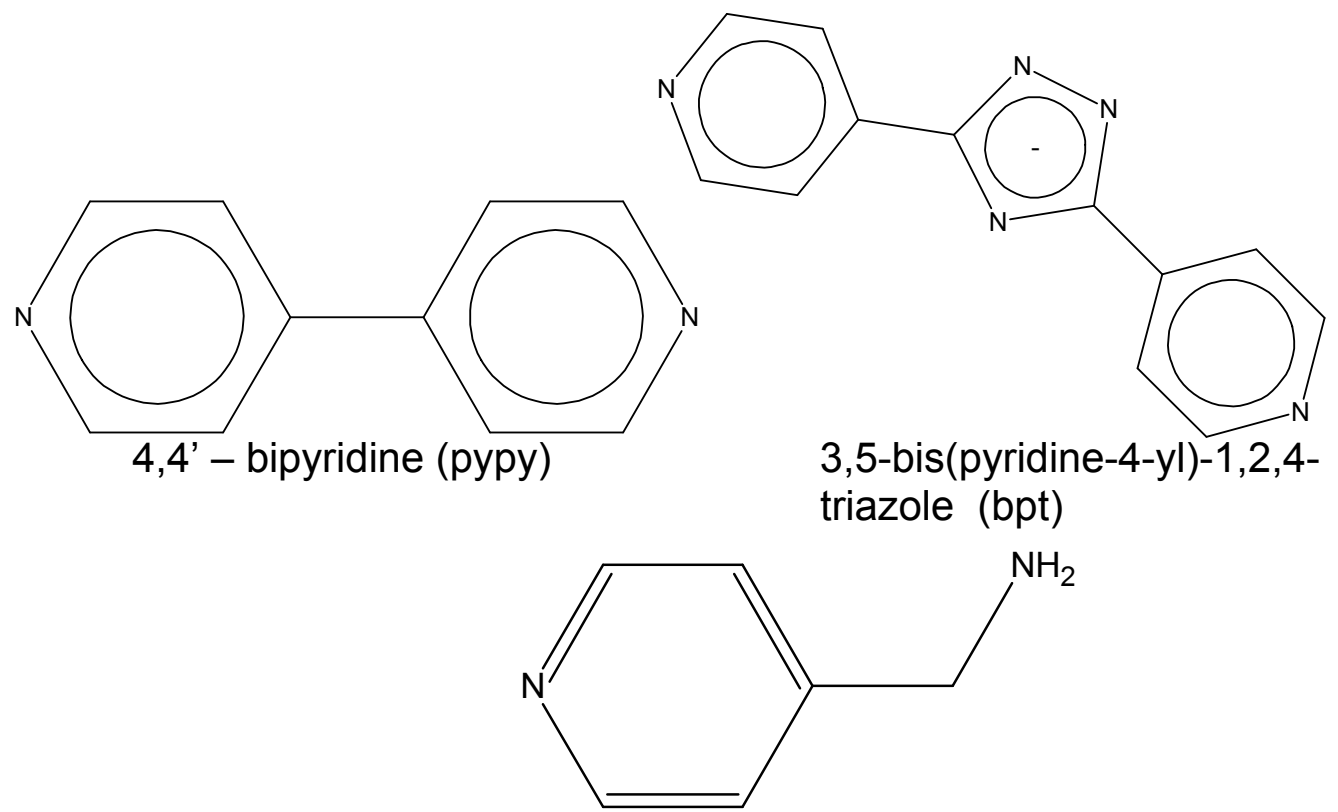

4-aminomethylpyridine (4-AMP)

Figure 4.1. 2 Various Pyridyl and Polypyridyl ligands 


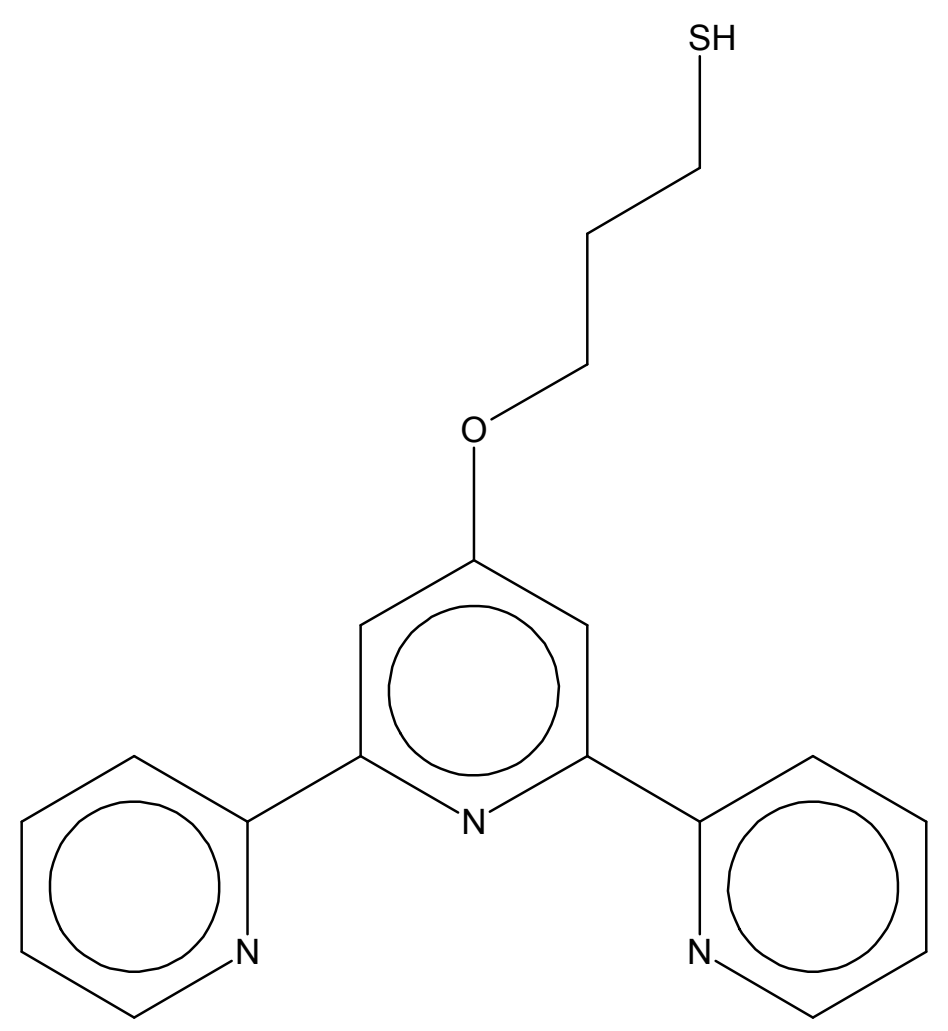

Figure 4.1. $3 \quad$ Dr. Mohler's Modified Terpyridine Ligand 


\subsection{Experimental - The Osmium System}

\subsubsection{Synthesis of $\left[\mathrm{Os}(\mathrm{tpy}-\mathrm{OH})(\mathrm{bpy}) \mathrm{OH}_{2}\right]\left(\mathrm{PF}_{6}\right)_{2}$}

It is important to note that because the modified terpyridine ligand was synthesized in-house, large quantities of it were not available. For this reason, it was important to successfully develop a method to synthesize $\left[\mathrm{Os}(\mathrm{tpy})(\mathrm{bpy}) \mathrm{OH}_{2}\right]^{2+/ 3+}$ first. The standard terpyridine ligand was commercially available and much cheaper to use. Once the development of the non-modified aqua complex was successful, the complex was synthesized using the modified terpyridine ligand. The following reaction schemes will be discussed with the non-modified terpyridine ligand. Incorporation of the tpy-OH ligand will be discussed in Section 4.2.3.5. CV's were performed in the indicated electrolytes at $0.1 \mathrm{~V} / \mathrm{s}$, unless otherwise specified.

\subsubsection{Synthesis of $\left[\mathrm{Os}(\mathrm{tpy})(\mathrm{bpy}) \mathrm{OH}_{2}\right]\left(\mathrm{PF}_{6}\right)_{2}$}

The synthesis of $\left.\mathrm{Os}(\mathrm{tpy})(\mathrm{bpy}) \mathrm{OH}_{2}\right]\left(\mathrm{PF}_{6}\right)_{2}$ was achieved via a multistep reaction according to the flowchart shown in Figure 4.2.1.1.1. Each step will be discussed separately. At each step, the reaction will be scaled to $100 \mathrm{mg}$ of Osmium-containing starting material, which was typical. All reactions contained a minimum of $50 \mathrm{mg}$ and a maximum of $200 \mathrm{mg}$ starting material. The amounts of the polypyridyl reagents will be discussed in terms of equivalents with respect to the Osmium starting 


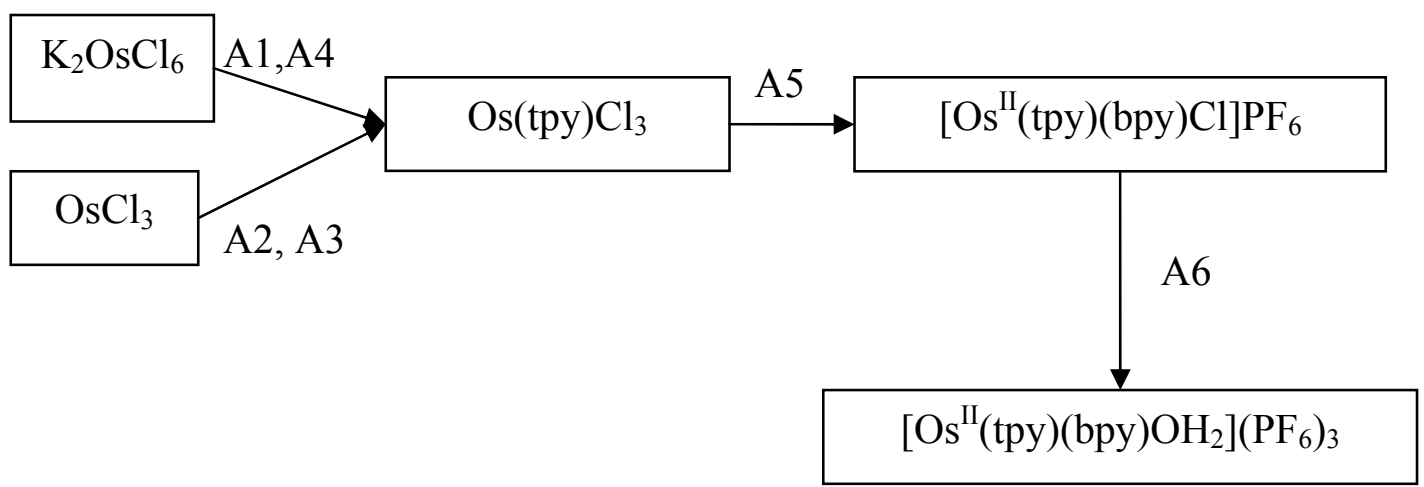

Figure 4.2.1.1. $1 \quad$ Flowchart of $\left[\mathrm{Os}(\mathrm{tpy})(\mathrm{bpy}) \mathrm{OH}_{2}\right]^{2+/ 3+}$ Synthesis. Synthesis pathways A1-A6 are discussed in the text. 
material, where relevant. Unless specified differently in the text, HPLC grade methanol and absolute ethanol were used as-purchased from Fisher, and N,Ndimethylformamide (DMF) was distilled over copper sulfate and stored over $4 \AA$ molecular sieves prior to use in order to remove water and decomposition products.

\subsection{Synthesis of $\mathrm{Os}(\mathrm{tpy}) \mathrm{Cl}_{3}$}

The synthesis of $\mathrm{Os}$ (tpy) $\mathrm{Cl}_{3}$ occurred via 4 pathways, two using $\mathrm{K}_{2} \mathrm{OsCl}_{6}$ as the starting material, and two using $\mathrm{OsCl}_{3} \cdot 3 \mathrm{H}_{2} \mathrm{O}$ as the starting material. Each path will be discussed separately.

A1. $\mathrm{K}_{2} \mathrm{OsCl}_{6} \rightarrow \mathrm{Os}(\mathrm{tpy}) \mathrm{Cl}_{3}$ (methanol synthesis)

This synthesis is taken from the literature ${ }^{79}$. $100 \mathrm{mg} \mathrm{K}_{2} \mathrm{OsCl}_{6}$ was combined with 1 equivalent (eq) terpyridine and $20 \mathrm{ml}$ methanol in a $50 \mathrm{ml}$ round bottom flask (r.b.f). The solution was refluxed at $65^{\circ} \mathrm{C}$ for $20 \mathrm{hrs}$. The solution was cooled, and a red solid was collected. According to Constable, the product should be black. Thus it was suspected that the replacement reaction did not occur. Voltammetry of both the solid product and a portion of the filtrate in DMF confirmed that $\mathrm{Os}(\mathrm{tpy}) \mathrm{Cl}_{3}$ had not formed. It is possible that the reflux temperature was not high enough to promote the reaction. An unpublished UV-VIS spectrum and cyclic voltammogram of Os(tpy) $\mathrm{Cl}_{3}$ (provided by Dr. Konstantino DeMadis) is shown in Figure 4.2.1.1.1.1. and Figure 4.2.1.1.1.2 


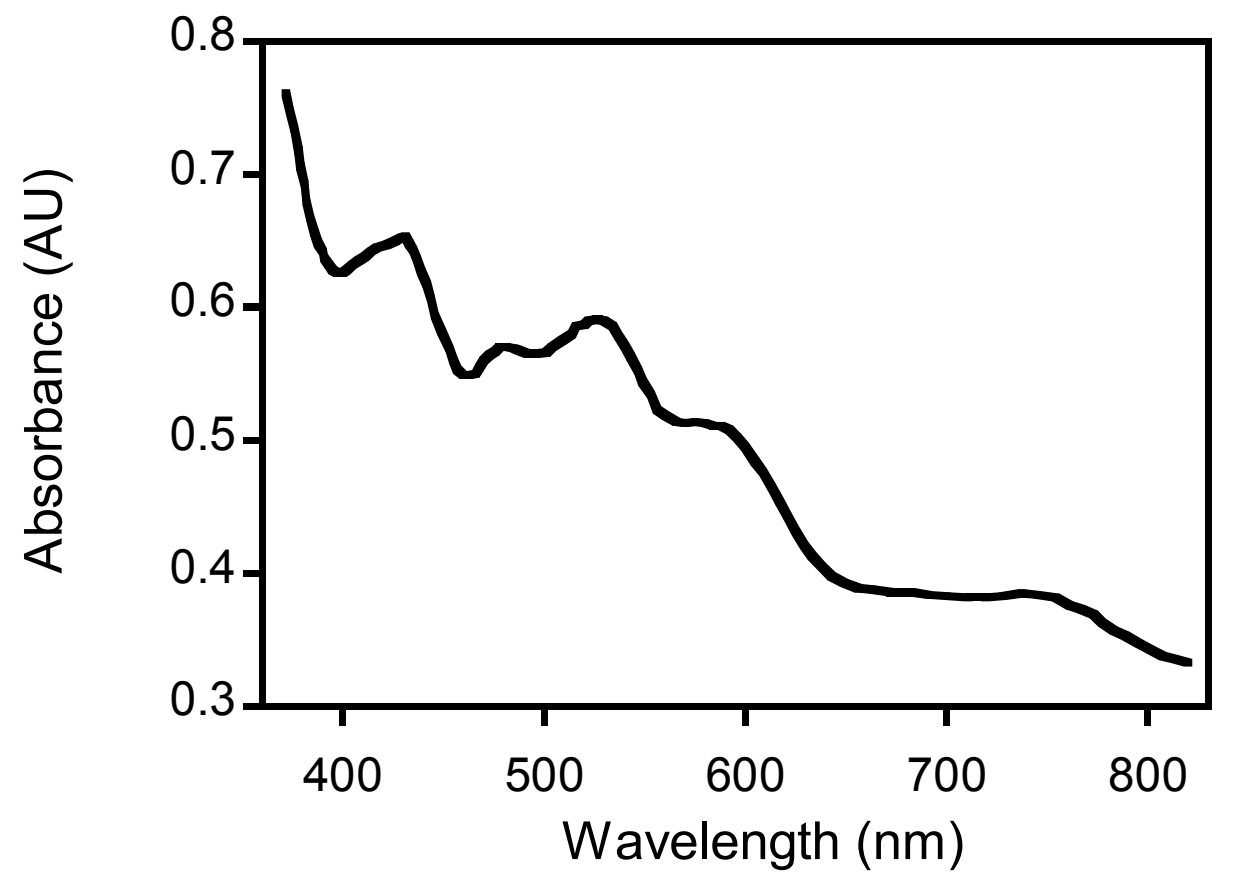

Figure 4.2.1.1.1. 1

Reference UV-VIS Spectrum of Os(tpy) $\mathrm{Cl}_{3}$ in $\mathrm{DMF}^{80}$ 

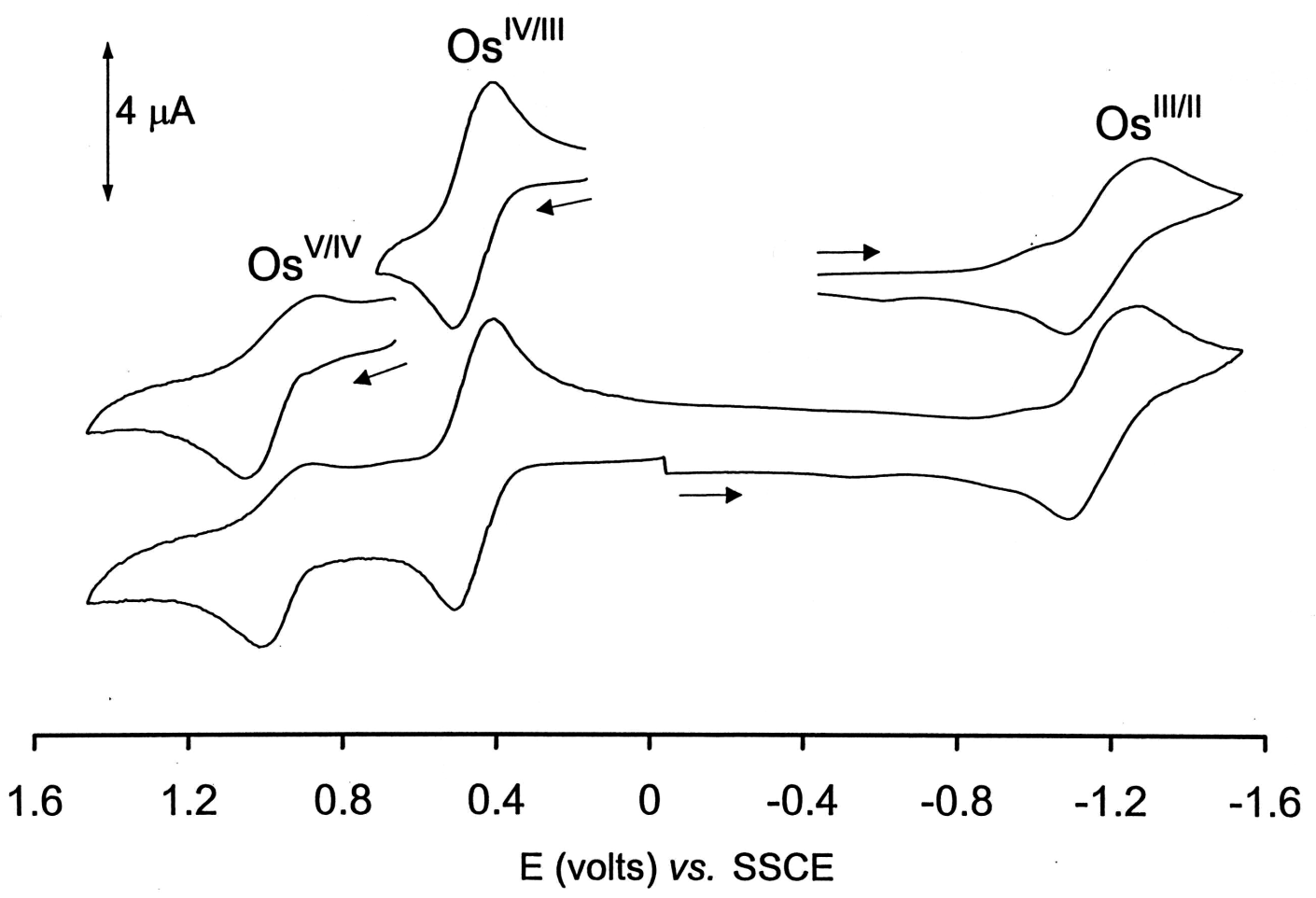

Figure 4.2.1.1.1. 2 Reference Voltammogram of $\mathrm{Os}(\mathrm{tpy}) \mathrm{Cl}_{3}$ in $0.1 \mathrm{M}$ tertbutylammonium hydroxide/DMF ${ }^{80}$ 
UV-VIS Spectrum (nm) - 420, 480, 530, 600, 740

Cyclic Voltammetry - CV's of both filtrate and filtrant were recorded. No significant electroactive waves in either CV.

It was apparent that neither the UV-VIS spectrum or the voltammogram were comparable to Demadis' data.

A2. $\mathrm{OsCl}_{3} \cdot 3 \mathrm{H}_{2} \mathrm{O} \rightarrow \mathrm{Os}($ tpy $) \mathrm{Cl}_{3}$

This procedure was provided by a previous student working on the project ${ }^{81}$. $100 \mathrm{mg} \mathrm{OsCl} \cdot 3 \mathrm{H}_{2} \mathrm{O}$ was combined with 1 eq terpyridine and $60 \mathrm{ml}$ ethanol in a $100 \mathrm{ml}$ r.b.f The mixture was refluxed at $78^{\circ} \mathrm{C}$ overnight. After cooling, an excess of ether was added, and a black solid was collected by filtration. The UV-VIS spectrum does not appear to match the spectrum of $\mathrm{Os}(\mathrm{tpy}) \mathrm{Cl}_{3}$.

UV-VIS Spectrum (nm) - 400, 520

A3. $\mathrm{OsCl}_{3} \cdot 3 \mathrm{H}_{2} \mathrm{O} \rightarrow \mathrm{Os}(\mathrm{tpy}) \mathrm{Cl}_{3}$

This approach was taken from a publication by Dwyer ${ }^{82} \cdot 100 \mathrm{mg} \mathrm{OsCl} 3 \cdot 3 \mathrm{H}_{2} \mathrm{O}$ is dissolved in $1 \mathrm{ml}$ DMF. The solution was heated to near boiling, then 1eq terpyridine was added. The mixture was refluxed for 15 minutes. The solution was cooled, and a black solid spontaneously precipitated. The solid was collected, then washed with cold DMF, followed by anhydrous ether. Initial experiments used distilled DMF as described 
above. The UV-VIS spectrum appears to have peaks around the proper positions, but it appears to be partially masked by impurities.

$$
\text { Yield - 64\% }
$$

UV-VIS Spectrum(nm) - 468, 534, 568, with extensive tailing

Later experiments used a modification of the published synthesis. First, high purity grade DMF was purchased which was sealed under a blanket of nitrogen in a sure-seal container, and dispensed with a syringe to minimize possible contamination. The DMF was stored in the refrigerator to minimize decomposition. While not a change in the synthesis step, the use of high grade DMF minimized the possibility of water contamination in the synthesis. As a further step to minimize the introduction of water, the reflux was performed under a flowing blanket of argon. The main procedural change was that the black solid was encouraged to precipitate with the addition of excess anhydrous ether. Also, the solid was washed extensively with distilled, deionized water. Washing with water removed a green water-soluble impurity. The product was washed until the washings turned brown. The UV-VIS spectrum of the product was identical to the one provided by DeMadis. Electrochemistry confirmed that there were no electroactive waves except for the two waves associated with $\mathrm{Os}(\mathrm{tpy}) \mathrm{Cl}_{3}$.

Typical Yield - 87\%

UV-VIS Spectrum(nm) - Figure 4.2.1.1.1.3. 428, 480, 524, 570, 750.

Cyclic Voltammetry(V vs. SCE) - Figure 4.2.1.1.1.4. 2 electroactive waves: Os(tpy) $\mathrm{Cl}_{3}$ waves at $\mathrm{E}_{1 / 2}=-0.28,+0.94$. 


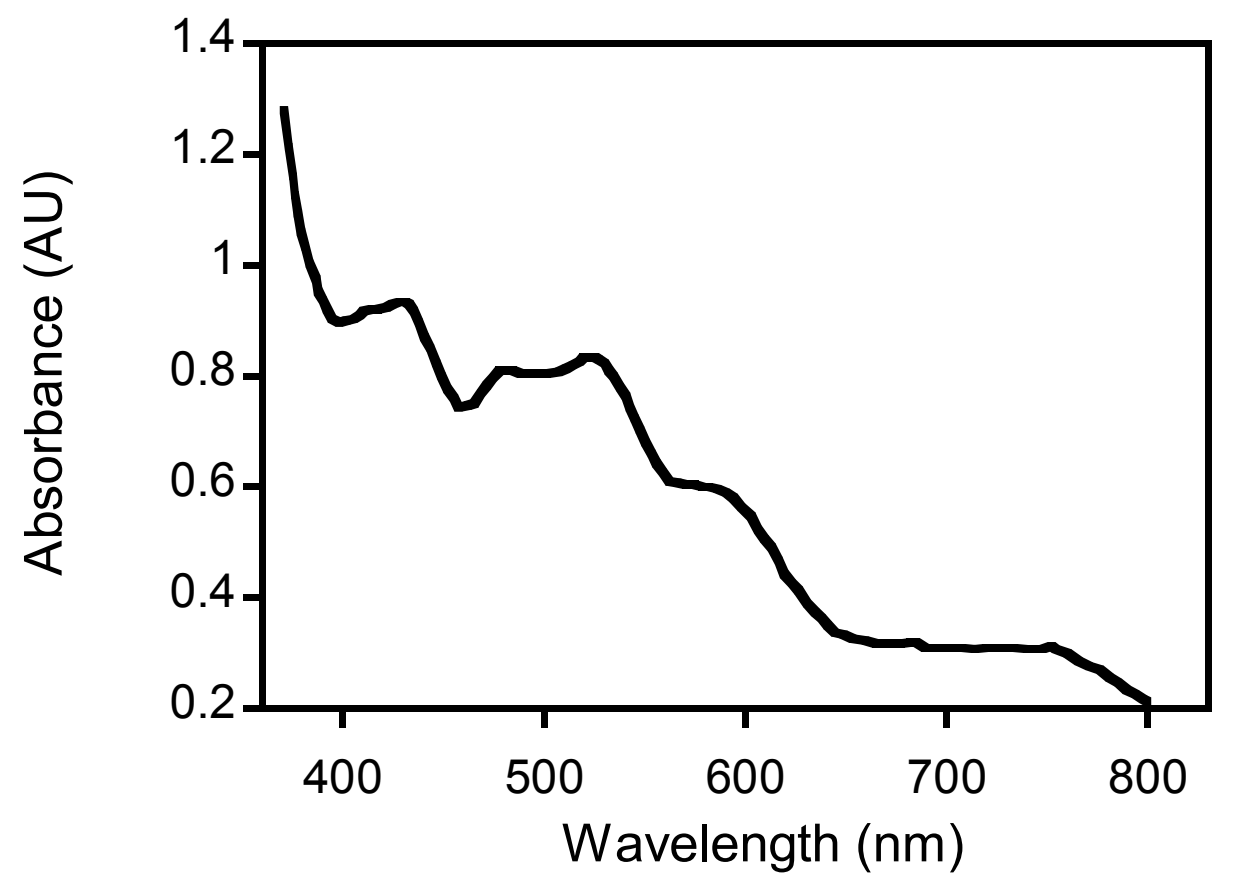

Figure 4.2.1.1.1. 3 UV-VIS Spectra of Os(tpy) $\mathrm{Cl}_{3}$ in DMF - Synthesis A3 with high purity grade DMF 


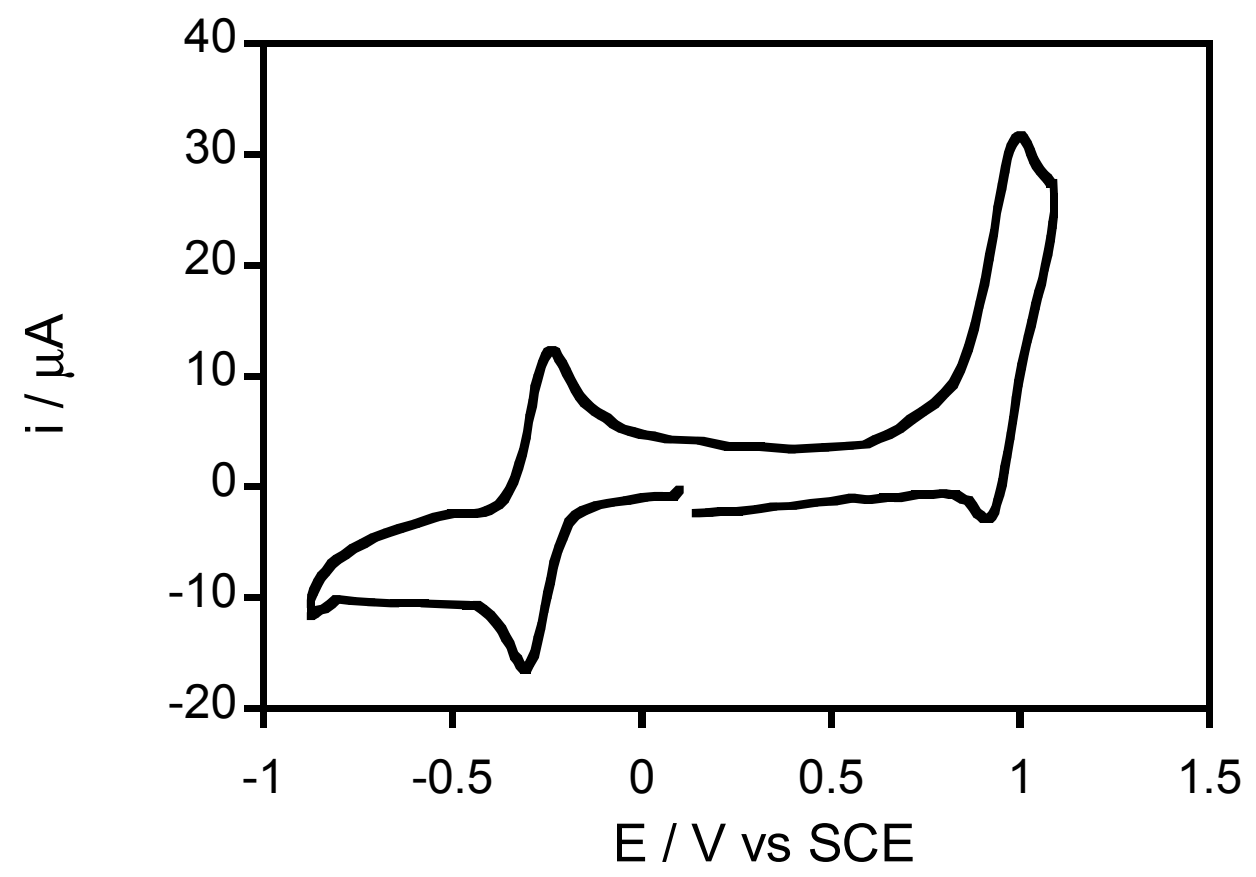

Figure 4.2.1.1.1. $4 \quad$ Voltammogram of $\mathrm{Os}(\mathrm{tpy}) \mathrm{Cl}_{3}$ in $0.1 \mathrm{M}$ tetramethylammonium tetrafluoroborate/DMF- Synthesis $A 3$ with high purity grade DMF. 
A4. $\mathrm{K}_{2} \mathrm{OsCl}_{6} \rightarrow \mathrm{Os}(\mathrm{tpy}) \mathrm{Cl}_{3}$ (DMF synthesis)

This procedure was taken from a publication by Buckingham ${ }^{83} .100 \mathrm{mg}(0.2 \mathrm{mmol})$ of $\mathrm{K}_{2} \mathrm{OsCl}_{6}$ was placed in a $50 \mathrm{ml}$ r.b.f., and dissolved in $2 \mathrm{ml}$ of DMF. 1 eq of $2,2^{\prime}: 6^{\prime}, 2^{\prime \prime}-$ terpyridine $(53 \mathrm{mg}, 0.2 \mathrm{mmol}$ ) was added to the r.b.f. Then, the mixture was refluxed at $153^{\circ} \mathrm{C}$ for two hours. The mixture was cooled, then filtered through a porous glass frit (medium grade). Potassium chloride $(\mathrm{KCl})$ crystals were collected in the frit and discarded. $2 \mathrm{ml}$ of absolute ethanol was added to the filtrate to promote the formation of crystals. Os(tpy) $\mathrm{Cl}_{3}$ was precipitated out of solution by the slow addition of an excess (typically 5 fold or greater) of anhydrous ether (ethyl ether). The black solid was collected by filtration and rinsed with copious quantities of distilled, deionized water, then anhydrous ether. Rinsing with water removed a green, water-soluble impurity and washing with ether facilitated drying of the product. The purity of the product was checked using thin-layer chromatography (TLC) and cyclic voltammetry. TLC using DMF as the solvent showed migration $95 \%$ of the product with the solvent front, with about $5 \%$ of the product barely migrating from the origin, using both silica gel and $\mathrm{C} 18$ reverse-phase silica gel plates. TLC using alumina plates showed product streaking across entire plate. A UV-VIS spectrum of the product showed the proper peaks. However, the spectrum is not very distinct, which suggests that some impurities are present. Voltammetry confirmed the presence of two minor electroactive peaks, in addition to the Os(tpy) $\mathrm{Cl}_{3}$ waves.

Typical Yield $-65 \%$

UV-VIS Spectrum(nm) - 425, 470, 520, 580, 755. 
Cyclic Voltammetry(V vs. SCE) - CV shows 5 electroactive waves: 2 minor impurity waves at $E_{1 / 2}=-0.63,+0.74$. Ferrocene reference at $E_{1 / 2}=+0.46$. Os(tpy) $\mathrm{Cl}_{3}$ waves at $\mathrm{E}_{1 / 2}=-0.29,+0.93$.

Purification - Recrystallization of product in DMF/ether+5\%EtOH, as per DeMadis $^{80}$. UV-VIS looks identical before and after recrystallization.

This procedure was modified in later experiments to minimize steps while achieving a reasonable amount of product. First, the high purity grade DMF was used (as discussed in method A3), and the reflux was performed under a blanket of argon. Also, the removal potassium chloride prior to precipitation of the product was omitted. The addition of ethanol to the solution prior to the addition of ether was removed as well. These two steps were removed primarily because they were unnecessary. Precipitation of the product occurred readily with the addition of ether alone, and the potassium chloride dissolved quickly during the washing step, leaving solid product only. Purity of the product was checked by cyclic voltammetry. CV's contained the two expected electroactive peaks, plus a small amount of one impurity peak.

Typical Yield $-65 \%$

Cyclic Voltammetry(V vs. SCE) - Figure 4.2.1.1.1.5. 1 minor unknown wave at $\mathrm{E}_{1 / 2}=+0.74$. Os $($ tpy $) \mathrm{Cl}_{3}$ waves at $\mathrm{E}_{1 / 2}=-0.29,+0.93$. 


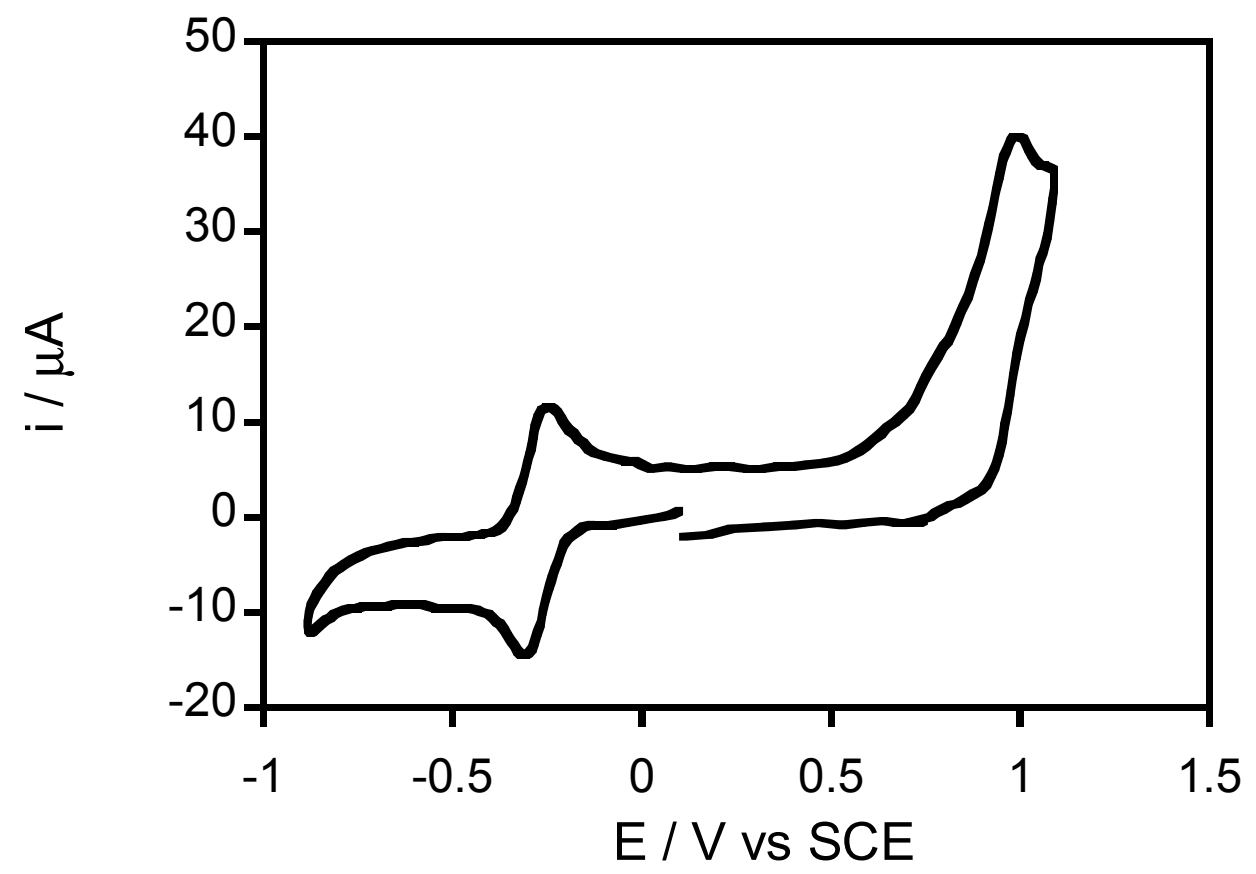

Figure 4.2.1.1.1. $5 \quad$ Voltammogram of $\mathrm{Os}(\mathrm{tpy}) \mathrm{Cl}_{3}$ in $0.1 \mathrm{M}$ tetramethylammonium tetrafluoroborate/DMF- Synthesis A4 with high purity grade DMF 


\section{Summary}

The formation of $\mathrm{Os}(\mathrm{tpy}) \mathrm{Cl}_{3}$ from either starting material is a simple ligand substitution reaction with a solvent reduction. I found that two main factors affected the success of the synthesis. First, the reactants needed be refluxed in an inert solvent at a temperature high enough to promote substitution of the terpyridine ligand. Reaction A1 likely failed because of the low reflux temperature of methanol. The second factor governing the success of the reaction appeared to be the purity of the solvent, especially with respect to the absence of water. It appears that water contamination will cause impurities. Throughout a large portion of the reactions attempted, the solvent used was distilled DMF that had been stored over molecular sieves. Apparently, this distillation procedure was not sufficient to remove all water content. After purchasing the high purity DMF, the impurities disappeared from the product. Both the A4 and A5 synthesis routes give similar results with the high purity DMF, with the synthesis from $\mathrm{OsCl}_{3} \cdot 3 \mathrm{H}_{2} \mathrm{O}$ yielding slightly cleaner voltammograms.

It is worth nothing that a published voltammogram of Os(tpy) $\mathrm{Cl}_{3}$ was not available early in method development. The unpublished UV-VIS spectrum from Dr. DeMadis provided the only reference to the $\mathrm{Os}(\mathrm{tpy}) \mathrm{Cl}_{3}$ product. This lack of published information might have resulted from problems in analyzing $\mathrm{Os}(\mathrm{tpy}) \mathrm{Cl}_{3}$ due to its high insolubility in conventional solvents. Os(tpy) $\mathrm{Cl}_{3}$ was moderately soluble only in DMF and dimethyl sulfoxide (DMSO). Voltammograms were collected in DMF. However, the analysis of the CV's was a bit indirect in determining which waves resulted from impurities and which waves resulted from $\mathrm{Os}(\mathrm{tpy}) \mathrm{Cl}_{3}$. The two major waves (at +0.30 
and $+.095 \mathrm{~V}$ vs. SCE ) were present in all CV's. However, the two minor waves (at 0.60 and $+0.75 \mathrm{~V}$ vs. SCE) were present only in products whose UV/VIS spectra showed significant deviation from the DeMadis reference. The two minor waves were absent from products whose spectra mirrored DeMadis' results. Thus, it was concluded that the two major waves were indeed due to $\mathrm{Os}(\mathrm{tpy}) \mathrm{Cl}_{3}$. This conclusion was also confirmed by the success of the next synthesis step. [Os(tpy)(bpy)Cl] $]^{2+/ 3+}$ has been well characterized and the good yields and purity of this step (which will be discussed shortly) suggest a successful synthesis of Os(tpy) $\mathrm{Cl}_{3}$. Demadis forwarded an advance copy of a publication which contained voltammetry information on Os(tpy)Cl $\mathrm{Cl}_{3}$ in late $1998^{80}$, which also confirmed that the two major waves seen in our spectra corresponded to $\mathrm{Os}(\mathrm{tpy}) \mathrm{Cl}_{3}$.

\subsection{Synthesis of $[\mathrm{Os}(\mathrm{tpy})(\mathrm{bpy}) \mathrm{Cl}]^{+/ 2+}$}

A5. Os(tpy) $\mathrm{Cl}_{3} \rightarrow\left[\mathrm{Os}\left(\right.\right.$ tpy)(bpy) $\mathrm{Cl}_{3} \mathrm{PF}_{6}$

The synthesis of $[\mathrm{Os}(\mathrm{tpy})(\mathrm{bpy}) \mathrm{Cl}]^{+}$is based on Buckingham's original synthesis paper from $1964^{83}$. Since then, a number of publications have synthesized similar compounds using the Buckingham paper as the foundation ${ }^{12 ; 39 ; 40 ; 67-69 ; 75 ; 84-90}$. In our synthesis, $100 \mathrm{mg}$ Os(tpy)Cl $\mathrm{Cl}_{3}$ was placed in a r.b.f., along with 1 equivalent bipyridine ligand $(29 \mathrm{mg})$ and $3 \mathrm{ml}$ of ethylene glycol. The mixture was refluxed for 1 hour under a blanket of argon to ensure complete substitution of the bpy ligand, although a color change (from brown to red) was noted after about 10 minutes under reflux. The 
solution was then cooled. $1 \mathrm{ml}$ of distilled, deionized water was added, and precipitation of the $\mathrm{PF}_{6}{ }^{-}$salt was initiated with the addition of $20-50 \mathrm{mg}$ solid $\mathrm{NH}_{4} \mathrm{PF}_{6}$.

Typical Yield > 90\%

UV-VIS Spectrum(nm) - Figure 4.2.1.1.2.1. 430, 520, 630, 800

Cyclic Voltammetry(V vs. SCE) - Figure 4.2.1.1.2.2. single wave at $+0.33 \mathrm{~V}$ No spectrum on $[\mathrm{Os}(\mathrm{tpy})(\mathrm{bpy}) \mathrm{Cl}]^{+}$was available. However, a spectrum of a similar compound $\left[\mathrm{Os}(\mathrm{bpy})_{2}(\mathrm{py}) \mathrm{Cl}\right]^{+}$had absorbance peaks at the following wavelengths ${ }^{78}$ $(\mathrm{nm})-427,500$, and 735 . These numbers roughly correspond to the spectrum of the compound.

Purification - Column chromatography using acidic alumina and acetonitrile. Red product band migrates, leaving an impurity band that sticks to the column until $\mathrm{MeOH}$ is added.

Summary

This synthesis was fairly straightforward. Comparison of the voltammetry (Figure 4.2.1.1.2.2) to Table 4.1.1 shows that the electroactive wave in the product agrees with the literature values. The purity of the product appeared to be mainly dependent on the quality of the starting material. Adsorption chromatography using alumina as the stationary phase was successful at removing impurities (which showed up in voltammetry around $+0.69 \mathrm{~V}$ vs. SCE). However, purification was largely unnecessary when using $\mathrm{Os}(\mathrm{tpy}) \mathrm{Cl}_{3}$ from a good synthesis batch. 


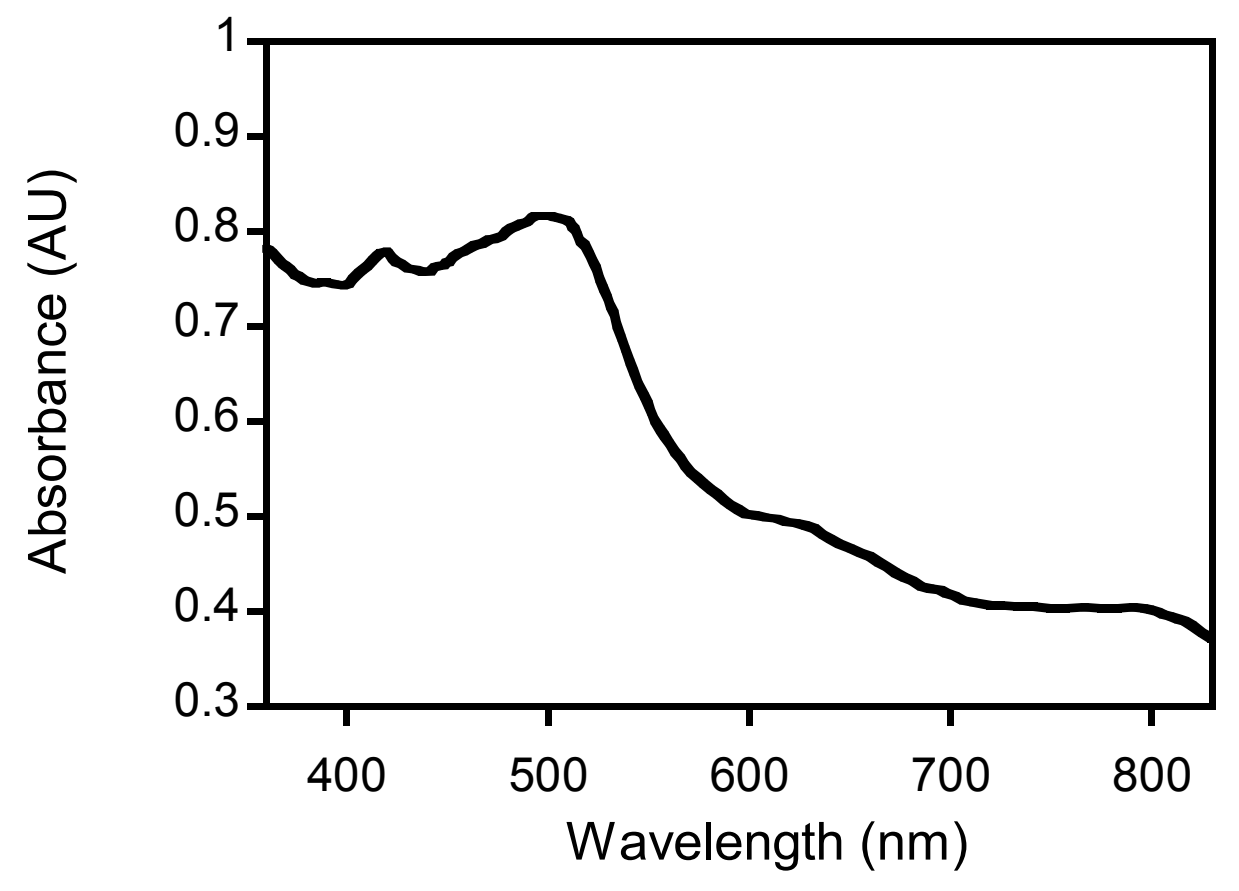

Figure 4.2.1.1.2. 1 UV-VIS Spectra of [Os(tpy)(bpy)CI]PF 6 in $\mathrm{CH}_{3} \mathrm{CN}-$ Synthesis A5 


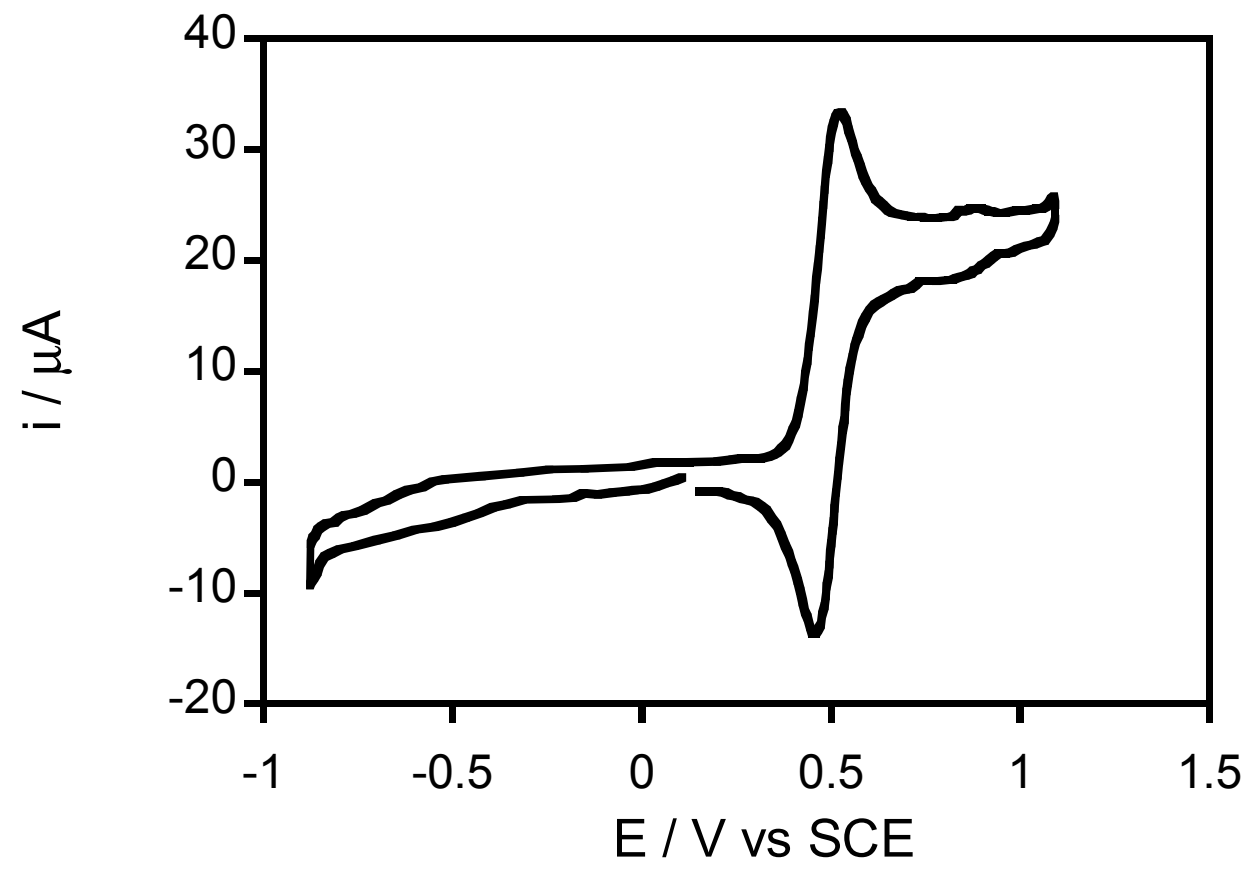

Figure 4.2.1.1.2. $2 \quad$ Voltammogram of [Os(tpy)(bpy)Cl]PF 6 in 0.1 $\mathrm{NaClO}_{4} / \mathrm{CH}_{3} \mathrm{CN}$ - Synthesis A5 


\subsection{Synthesis of $\left[\mathrm{Os}(\mathrm{tpy})(\mathrm{bpy}) \mathrm{OH}_{2}\right]^{2+/ 3+}$}

A6. Os(tpy)(bpy)Cl]PF $\rightarrow\left[\mathrm{Os}(\right.$ tpy $)($ bpy $\left.) \mathrm{OH}_{2}\right]\left(\mathrm{PF}_{6}\right)_{2}$

The synthesis of $\left[\mathrm{Os}(\mathrm{tpy})(\mathrm{bpy}) \mathrm{OH}_{2}\right]^{2+/ 3+}$ was first attempted using a method published by Meyer ${ }^{66}$. $100 \mathrm{mg}\left[\mathrm{Os}(\mathrm{tpy})(\mathrm{bpy}) \mathrm{Cl}_{\mathrm{PF}} \mathrm{F}_{6}\right.$ was added to $1.5 \mathrm{ml}$ concentrated trifluoromethanesulfonic acid (triflic acid). Under a blanket of argon, the mixture was heated to $110^{\circ} \mathrm{C}$ for $2 \mathrm{hr}$ in an oil bath. The rigorous conditions substituted the chloride with the readily displaceable triflate anion $\left(\mathrm{CF}_{3} \mathrm{SO}_{3}{ }^{-}\right)$. The solution was cooled and $2.5 \mathrm{ml}$ distilled, deionized water was added along with a small amount of freshly prepared Zinc amalgam. Zinc amalgam was prepared by placing Zinc chunks in a $1 \mathrm{M}$ $\mathrm{HNO}_{3}$ solution for 15 minutes to clean the Zinc. The Zinc was removed, rinsed thoroughly with distilled, deionized water, and added to a dilute solution of $\mathrm{Hg}\left(\mathrm{NO}_{3}\right)_{2}$ for 15 minutes. The newly formed zinc amalgam was rinsed at least 4 times with deionized, distilled water, then immediately placed in the triflic acid solution. The solution was stirred for 3 hours to ensure complete reduction of the Osmium product to the $\mathrm{Os}^{2+}$ oxidation state. The amalgam was removed by filtration. A small amount $(<$ $1 \mathrm{ml}$ ) of saturated $\mathrm{NH}_{4} \mathrm{PF}_{6}$ was added to the filtrate, and the precipitate was collected via filtration and rinsed thoroughly with distilled, deionized water. Since the osmiumaqua complex is a $1 \mathrm{e} 1 \mathrm{H}$ system, the formal potential is dependent on $\mathrm{pH}$. Thus voltammetry must be performed over a range of pH's to characterize the complex. 
Typical Yield > 80\%

UV-VIS Spectrum(nm) - 500, 600, 780

Cyclic Voltammetry(V vs. SCE) - correct pH dependency, significant impurity In all initial syntheses, the osmium aqua complex was impure. Both the UV-VIS spectra and the voltammograms contained evidence for significant impurities. A number of purification techniques were attempted with little success. Adsorption chromatography using acetone/acetonitrile/methanol as eluents was successful at separating bands from the product, but upon investigation, all bands were $\mathrm{pH}$ independent. It was thought that something (perhaps residual chloride) displaced the water group from the complex during chromatography. Ion-exchange chromatography was used by Meyer for the separation of [Os(tpy)(bpy)OH $\left.\mathrm{OH}_{2}\right]\left(\mathrm{PF}_{6}\right)_{2}$, using Sephadex SP120 with $0.1 \mathrm{M} \mathrm{NaClO}_{4}$ as the eluent ${ }^{66}$. SP-120 was unavailable, but chromatography was performed using SP-25 and CM-25 Sephadex. The number value relates to the crosslinking of the gel, with SP-25 having a higher percentage of crosslinking than SP120. 1:1 acetic acid/water mix was used as the mobile phase. Purification was unsuccessful using SP-25; no bands would elute. However, using CM-25, the osmium aqua product was separated as a brown band. It was determined that purification was unnecessary when using triflic acid that had been freshly distilled over toluene, a procedure taken from the literature ${ }^{84}$. Syntheses using distilled triflic acid yielded a product which did not require further purification.

UV-VIS Spectrum(nm) - Figure 4.2.1.1.3.1.

Cyclic Voltammetry(V vs. SCE) - Figure 4.2.1.1.3.2. 


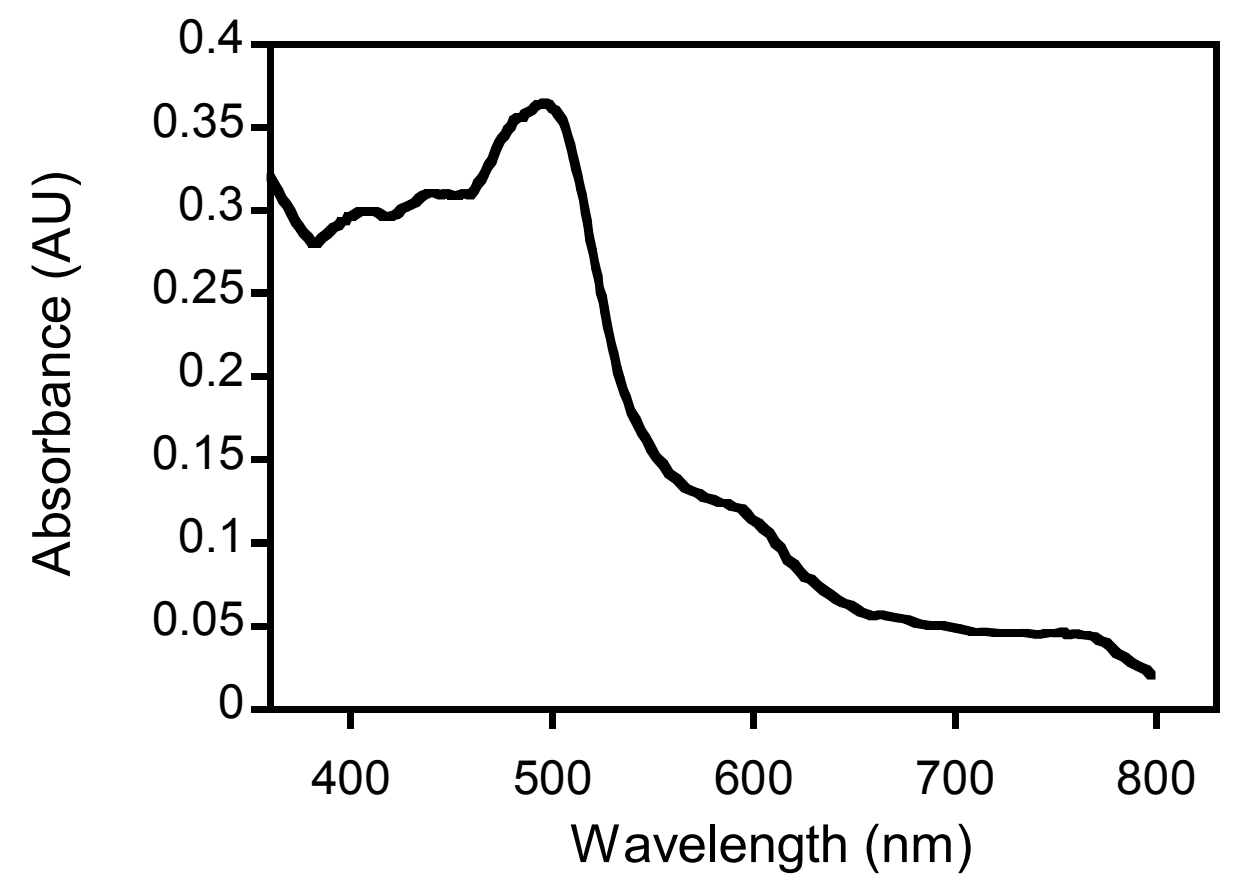

Figure 4.2.1.1.3. $1 \quad$ UV-VIS Spectra of $\left[\mathrm{Os}(\mathrm{tpy})(\mathrm{bpy}) \mathrm{OH}_{2}\right]\left(\mathrm{PF}_{6}\right)_{2}$ in $\mathrm{H}_{2} \mathrm{O}$ Synthesis A6 using Distilled TFMS acid 


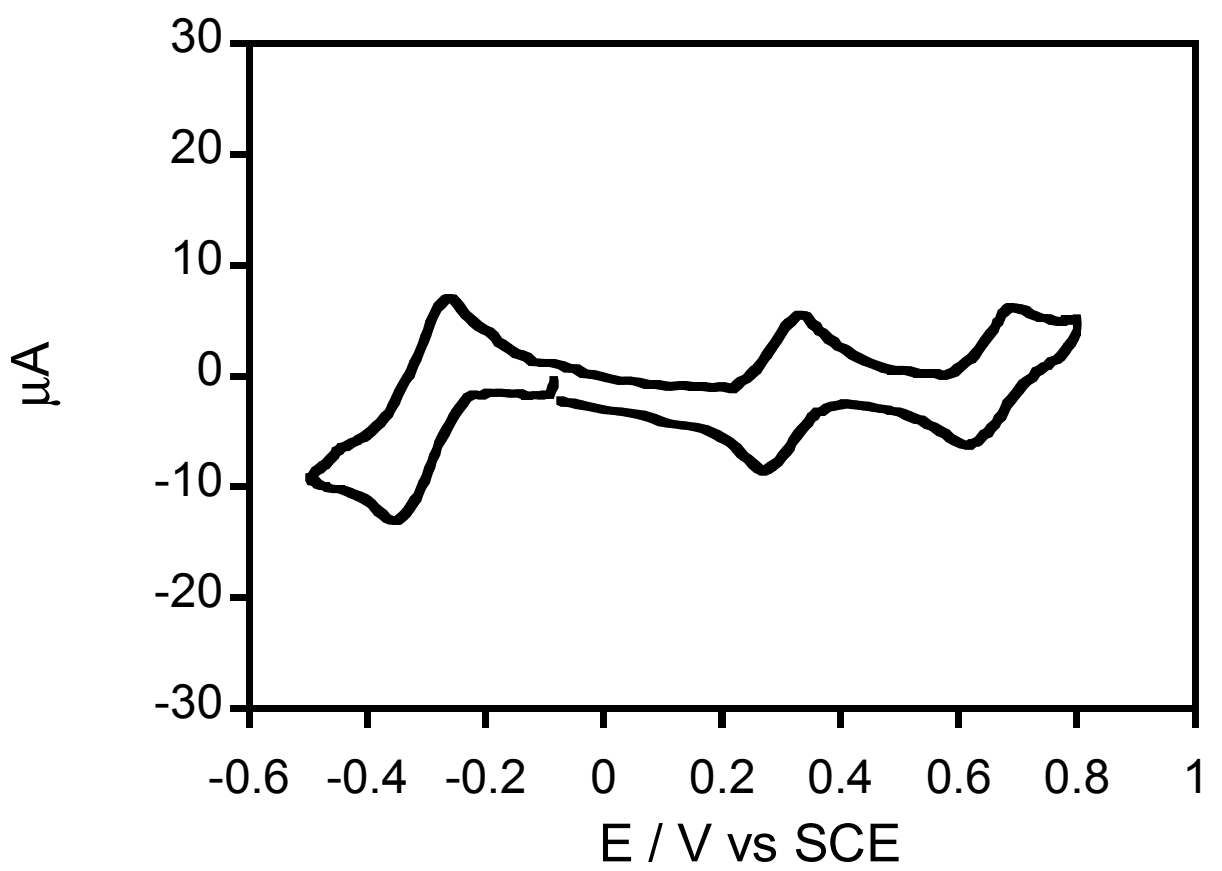

Figure 4.2.1.1.3. 2 Voltammogram of $\left[\mathrm{Os}(\mathrm{tpy})(\mathrm{bpy}) \mathrm{OH}_{2}\right]\left(\mathrm{PF}_{6}\right)_{2}$ in $0.1 \mathrm{M}$ $\mathrm{H}_{2} \mathrm{SO}_{4}+0.1 \mathrm{M} \mathrm{H}_{3} \mathrm{PO}_{4}$ aqueous solution at $\mathrm{pH} 3$ - Synthesis $\mathrm{A6}$ using Distilled TFMS acid 
Figure 4.2.1.1.3.3 shows the dependence of $\mathrm{E}_{1 / 2}$ of the $\mathrm{Os}^{2+/ 3+}$ wave on $\mathrm{pH}$. The fit of the data yields $\mathrm{pK}_{\mathrm{a} 1}=2.1$ and $\mathrm{pK}_{\mathrm{a} 2}=7.9$, which is in agreement with the data published by Meyer ${ }^{66}$.

\subsubsection{Synthesis of the modified terpyridine ligand}

With the successful synthesis of $\left[\mathrm{Os}(\mathrm{tpy})(\mathrm{bpy}) \mathrm{OH}_{2}\right]\left(\mathrm{PF}_{6}\right)_{2}$, it was necessary to incorporate the modified tpy ligand into the synthesis. Prof. Mohler had provided a small amount of the tpy-OH ligand along with a significant amount of tpy=O, a precursor to the tpy- $\mathrm{OH}$ ligand (where the tether was simply an oxygen at the 4 position of the middle ring). In order to provide enough tpy-OH ligand, more ligand was synthesized using a procedure provided by Prof. Mohler. $500 \mathrm{mg}$ tpy=O and $1.66 \mathrm{~g}$ $\mathrm{K}_{2} \mathrm{CO}_{3}$ were placed in a $100 \mathrm{ml}$ r.b.f. $68 \mathrm{ml}$ acetonitrile was added, followed by $0.18 \mathrm{ml}$ of 3-bromopropanol. The mixture was heated to $60^{\circ} \mathrm{C}$ and stirred overnight. The solution was cooled and the solvent was removed in vacuo with as little heating as possible. The residue was dissolved in $100 \mathrm{ml}$ methylene chloride and extracted with $100 \mathrm{ml}$ saturated sodium chloride solution. The aqueous layer was washed twice with methylene chloride, and all three organic portions were combined. The methylene chloride was dried with the addition of magnesium sulfate. The magnesium sulfate was filtered off and the filtrate was dried in vacuo. Next,the solid was washed with copious amounts of ether to remove any nopolar impurities. The tpy-OH product was characterized with proton NMR. The $\mathrm{H}^{1}-\mathrm{NMR}$ of the tpy-OH product is given in Figure

\subsubsection{1.}




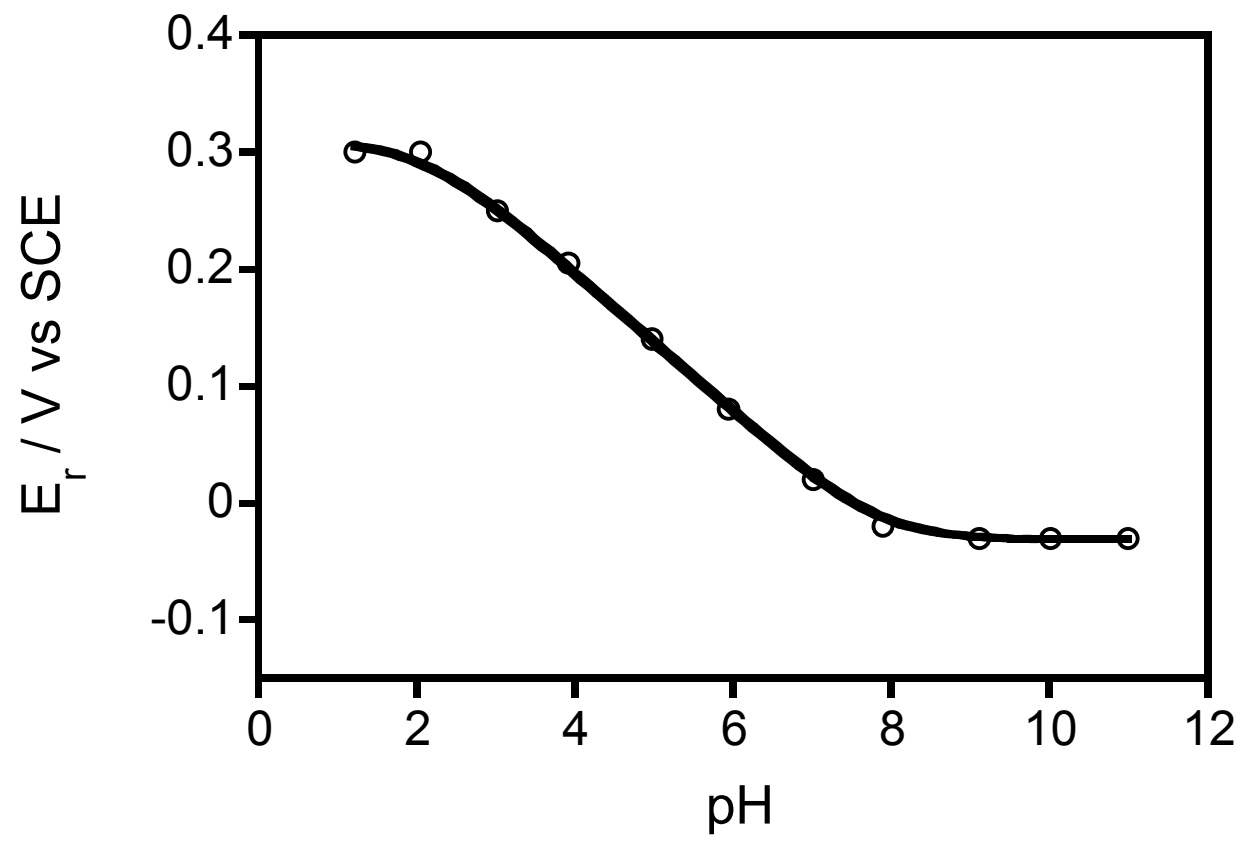

Figure 4.2.1.1.3. 3

E1/2 vs pH - [Os(tpy)(bpy)OH $\left.{ }_{2}\right]^{2+}$ 


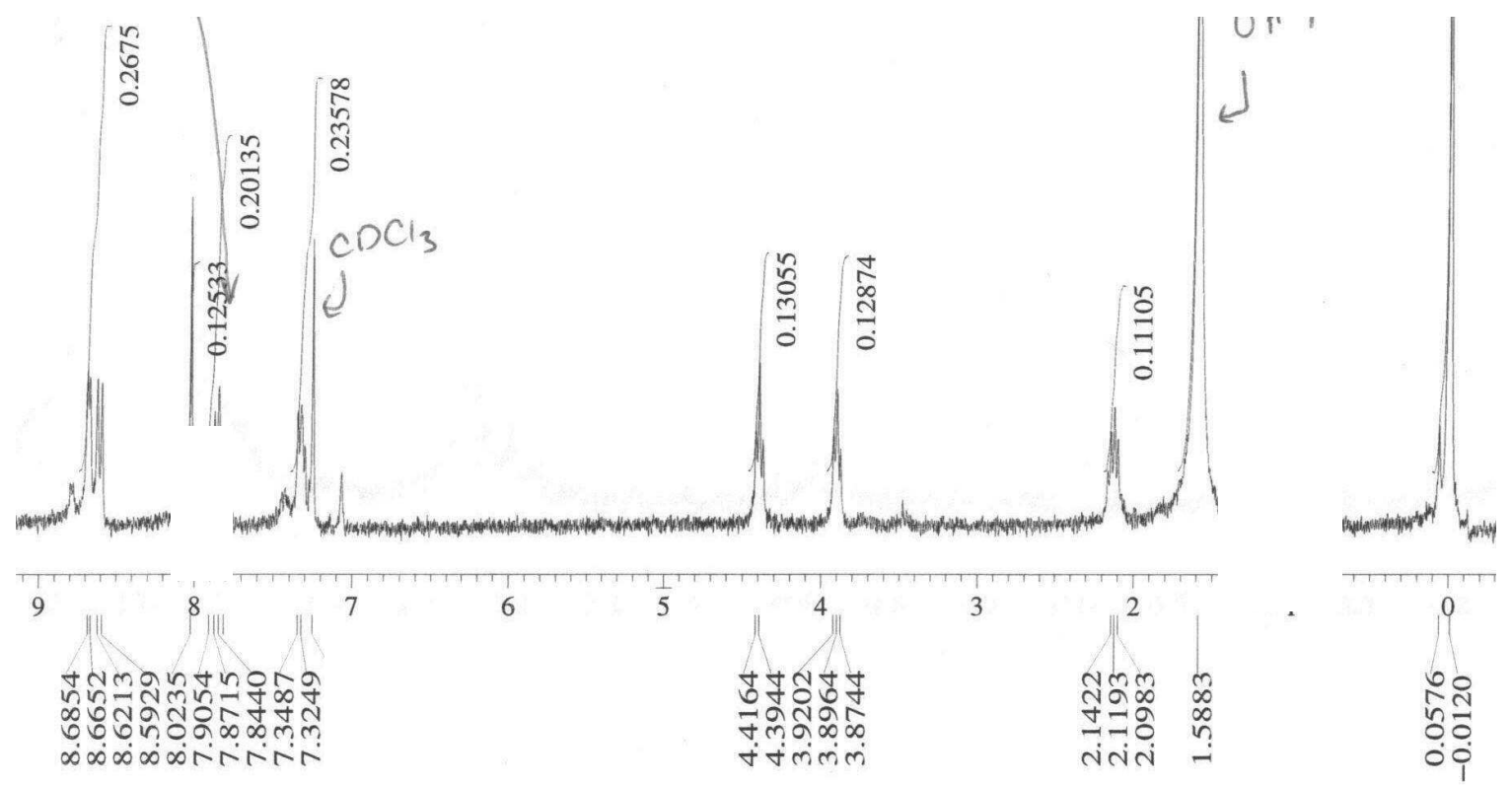

Figure 4.2.1.2. 1

${ }^{1} \mathrm{H}-\mathrm{NMR}$ of tpy-OH in deuterated methanol 
Yield - 15\%

$H^{1}$-NMR - Figure 4.2.1.2.1.

The yield in this reaction is low because the tpy=0 precursor is quite impure. Dr.

Mohler had found that the synthesis of tpy-OH could be completed without purification of the tpy=O material.

\subsubsection{Synthesis of $[\mathrm{Os}(\mathrm{tpy}-\mathrm{OH})(\mathrm{bpy}) \mathrm{Cl}]^{2+/ 3+}$ and $\left[\mathrm{Os}(\mathrm{tpy}-\mathrm{OH})(\mathrm{bpy}) \mathrm{OH}_{2}\right]^{2+/ 3+}$}

The synthesis of $\mathrm{Os}(\mathrm{tpy}-\mathrm{OH}) \mathrm{Cl}_{3}$ was achieved using the $\mathrm{A} 3$ pathway. The only modification to the synthesis was that 1 equivalent of tpy-OH was substituted for the tpy ligand. $\mathrm{Os}(\mathrm{tpy}-\mathrm{OH}) \mathrm{Cl}_{3}$ was synthesized in reasonable yield and purity

Typical Yield > 80\%

Cyclic Voltammetry(V vs. SCE) - Figure 4.2.1.3.1.

The synthesis of $\left[\mathrm{Os}(\mathrm{tpy}-\mathrm{OH})(\mathrm{bpy}) \mathrm{Cl}_{\mathrm{PF}} \mathrm{P}_{6}\right.$ proceeded throught the A5 pathway as described above, using $\mathrm{Os}(\mathrm{tpy}-\mathrm{OH}) \mathrm{Cl}_{3}$ as the starting material. This synthesis gave a good yield and a reasonably pure product.

Typical Yield > 90\%

Cyclic Voltammetry(V vs. SCE) - Figure 4.2.1.3.2.

Mass Spectrum - Figure 4.2.1.3.3

It was important to determine if the alcohol-terminated tether had survived the synthesis. A mass spectrum was collected with the help of Gregg Pratt using a LC/MS 


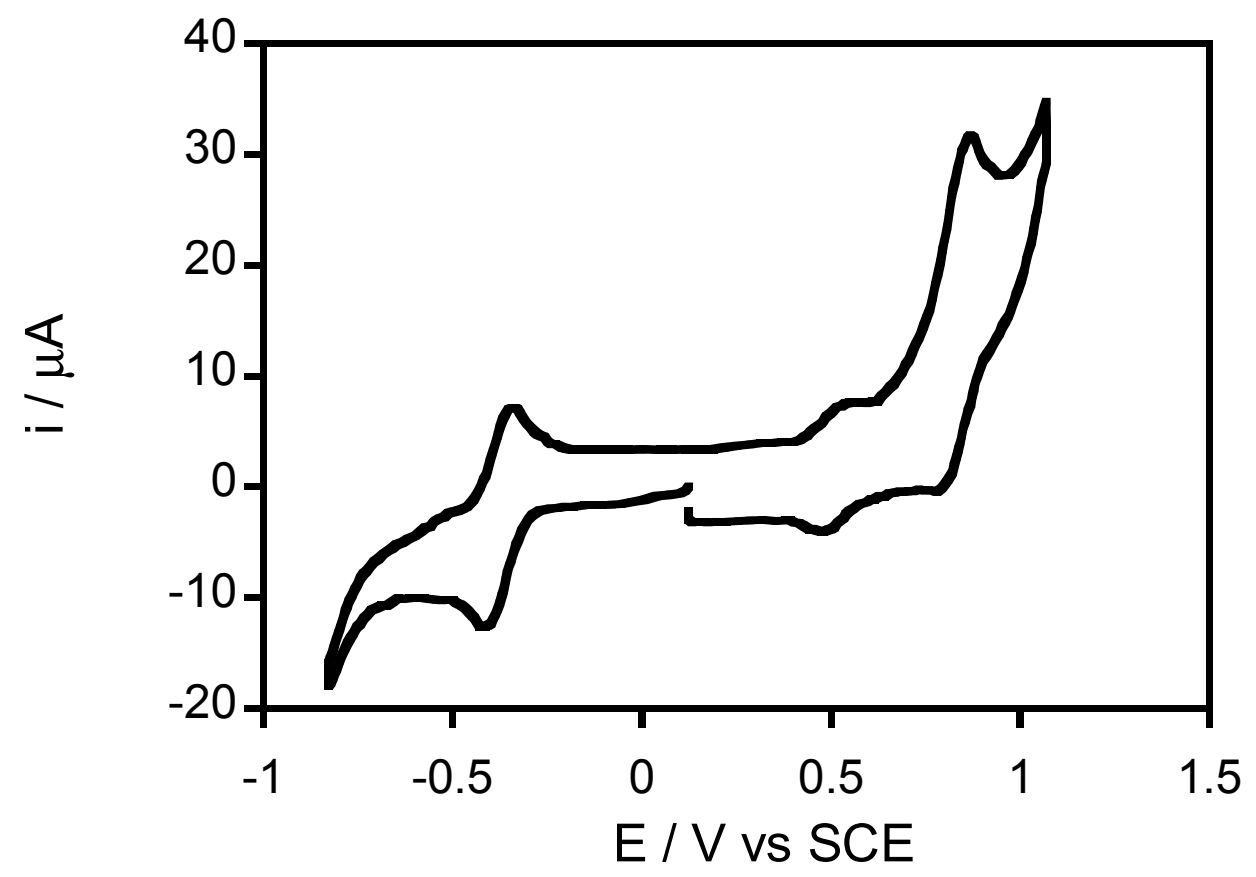

Figure 4.2.1.3. $1 \quad$ Voltammogram of $\mathrm{Os}(\mathrm{tpy}-\mathrm{OH}) \mathrm{Cl}_{3}$ in $0.1 \mathrm{M}$ tetramethylammonium tetrafluoroborate/DMF 


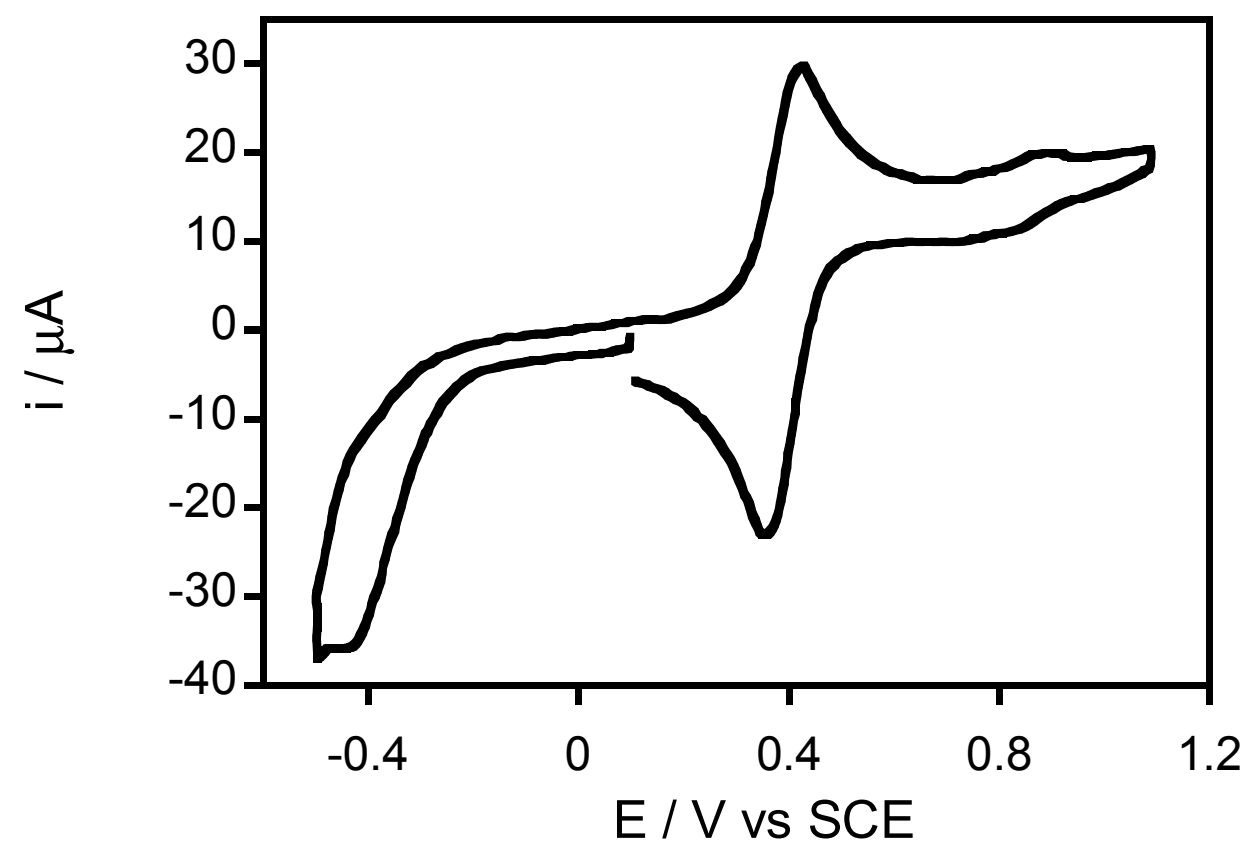

Figure 4.2.1.3. 2

$\mathrm{NaClO}_{4} / \mathrm{CH}_{3} \mathrm{CN}$

Voltammogram of [Os(tpy-OH)(bpy)Cl]PF 6 in $0.1 \mathrm{M}$ 


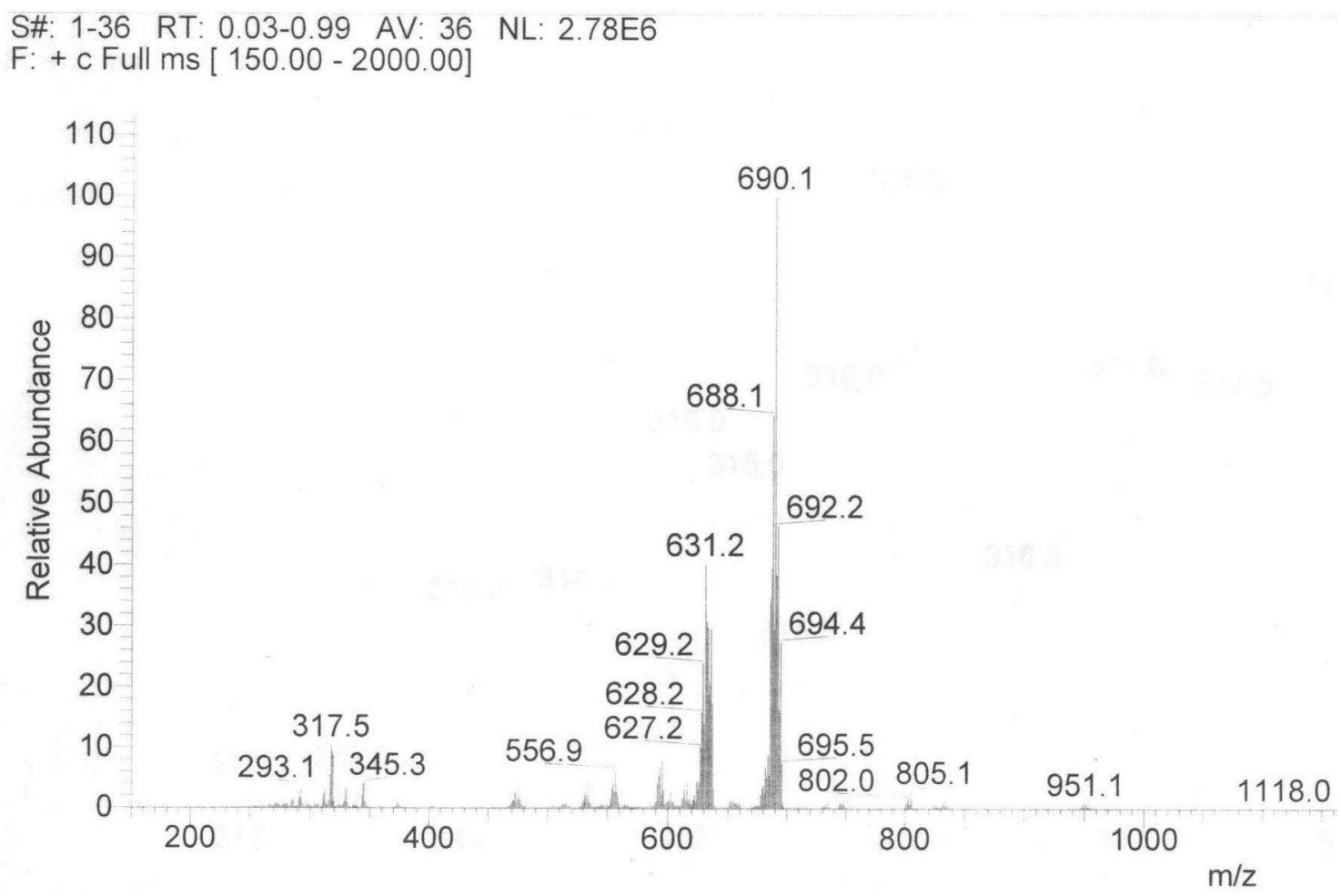

Figure 4.2.1.3. 3

Mass Spectrum of [Os(tpy-OH)(bpy)Cl] $\mathrm{PF}_{6}$ in $\mathrm{CH}_{3} \mathrm{CN}$ 
with an electrospray interface. Results are seen in Figure 4.2.1.3.3. [Os(tpy$\mathrm{OH})(\mathrm{bpy}) \mathrm{Cl}]^{+}$has a $\mathrm{m} / \mathrm{z}=689.22$. Experimentally, the major parent peak in the $\mathrm{MS}$ appeared at $\mathrm{m} / \mathrm{z}=690.1$. These two numbers are sightly different. It turns out that this discrepancy is due to a shift in the $\mathrm{m} / \mathrm{z}$, associated with the isotopic distribution of the compound. Figure 4.2.1.3.4 compares the calculated distribution with the experimental distribution taken from the spectrum. Both distributions have $\mathrm{m} / \mathrm{z}=690$ for the main peak, with good correlation between the minor peaks. Thus, it was concluded that the synthesis of $[\mathrm{Os}($ tpy-OH)(bpy)Cl]PF 6 was successful.

The synthesis of $\left[\mathrm{Os}(\mathrm{tpy}-\mathrm{OH})(\mathrm{bpy}) \mathrm{OH}_{2}\right]\left(\mathrm{PF}_{6}\right)_{2}$ was achieved using the $\mathrm{A} 6$ synthesis pathway, using $\left[\mathrm{Os}(\mathrm{tpy}-\mathrm{OH})(\mathrm{bpy}) \mathrm{Cl}_{\mathrm{P}} \mathrm{PF}_{6}\right.$ as the starting material. The only change that was made was to the reduction step with Zinc amalgam. This was removed in favor of reduction by electrolysis, which removed the possibility of Zinc contamination. After heating the solution at $110^{\circ} \mathrm{C}$ for 1 hour, the reflux condenser was removed and $95 \%$ of the triflic acid was allowed to evaporate. The residue was cooled and $10 \mathrm{ml}$ of $.1 \mathrm{M} \mathrm{H}_{2} \mathrm{SO}_{4} / \mathrm{H}_{3} \mathrm{PO}_{4}$ was added to dissolve the residue. The solution was placed in an electrochemical cell with a gold mesh working electrode and a platinum mesh counter electrode to allow for large currents. The cell was turned on at the lowest current sensitivity, and the potential was held at $0.0 \mathrm{~V}$ vs SCE, which was sufficient to reduce the complex to its +2 form. The solution was rotovapped to remove $90 \%$ of the solution and the product was precipitated with the addition of a small amount of $\mathrm{NH}_{4} \mathrm{PF}_{6}$. 


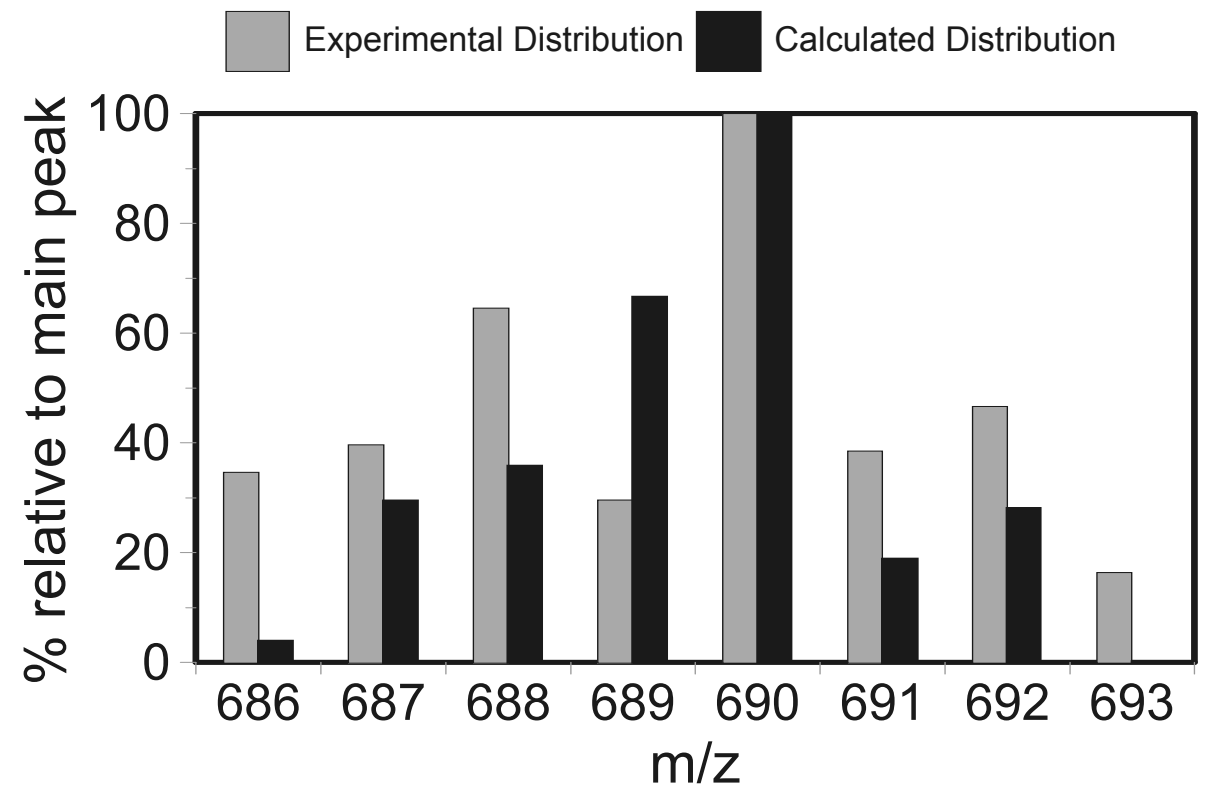

Figure 4.2.1.3. 4

Isotopic Distribution of [Os(tpy-OH)(bpy)Cl] $\mathrm{PF}_{6}$ 
Typical Yield $-50 \%$

UV-VIS Spectrum - Figure 4.2.1.3.5

Cyclic Voltammetry(V vs. SCE) - Figure 4.2.1.3.6.

Mass Spectrum - Figure 4.2.1.3.7

Yields were relatively low, and voltammograms appeared to contain impurities.

Chromatography using SP-25 and CM-25 was unsuccessful at removing impurities. More important, however, was the data from the mass spectrum. [Os(tpy-

$\left.\mathrm{OH})(\mathrm{bpy}) \mathrm{OH}_{2}\right]^{2+}$ should have an approximate $\mathrm{m} / \mathrm{z}=667.1$. This peak is absent from the spectrum. The main parent peak is around $\mathrm{m} / \mathrm{z}=613.2$. This peak would correspond to the complex after removal of the propanol tether. This complex, [Os(tpyO)(bpy) $\left.\mathrm{OH}_{2}\right]^{\mathrm{n+}}$ would have a $\mathrm{m} / \mathrm{z}=612.8$, assuming a negative charge on the oxygen attached to the terpyridine, or a $\mathrm{m} / \mathrm{z}=306.4$ if the oxygen was in radical form. With the $1 \mathrm{~m} / \mathrm{z}$ shift due to the isotopic distribution shift of the main peak (as seen earlier), the mass spectrum is consistent with $\left[\mathrm{Os}\left(\mathrm{tpy}-\mathrm{O}^{-}\right)(\mathrm{bpy}) \mathrm{OH}_{2}\right]$. Unfortunately this means that the tether was lost somewhere in the A6 synthesis step. It is likely that it was lost due to the vigorous conditions (exposure to hot triflic acid) necessary in removing the chloride from the complex. 


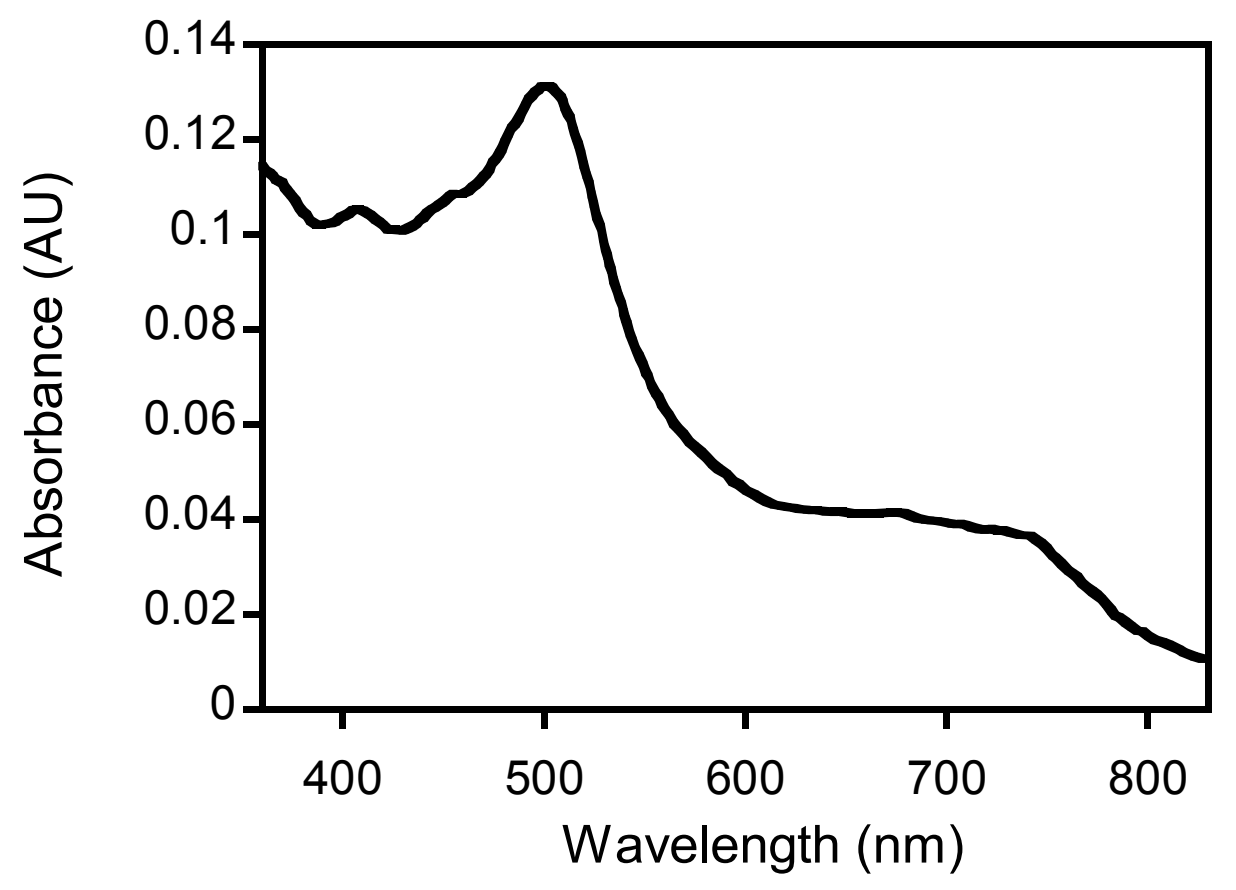

Figure 4.2.1.3. 5 UV-VIS of [Os(tpy-OH)(bpy) $\left.\mathrm{OH}_{2}\right]\left(\mathrm{PF}_{6}\right)_{2}$ in $\mathrm{H}_{2} \mathrm{O}$ 


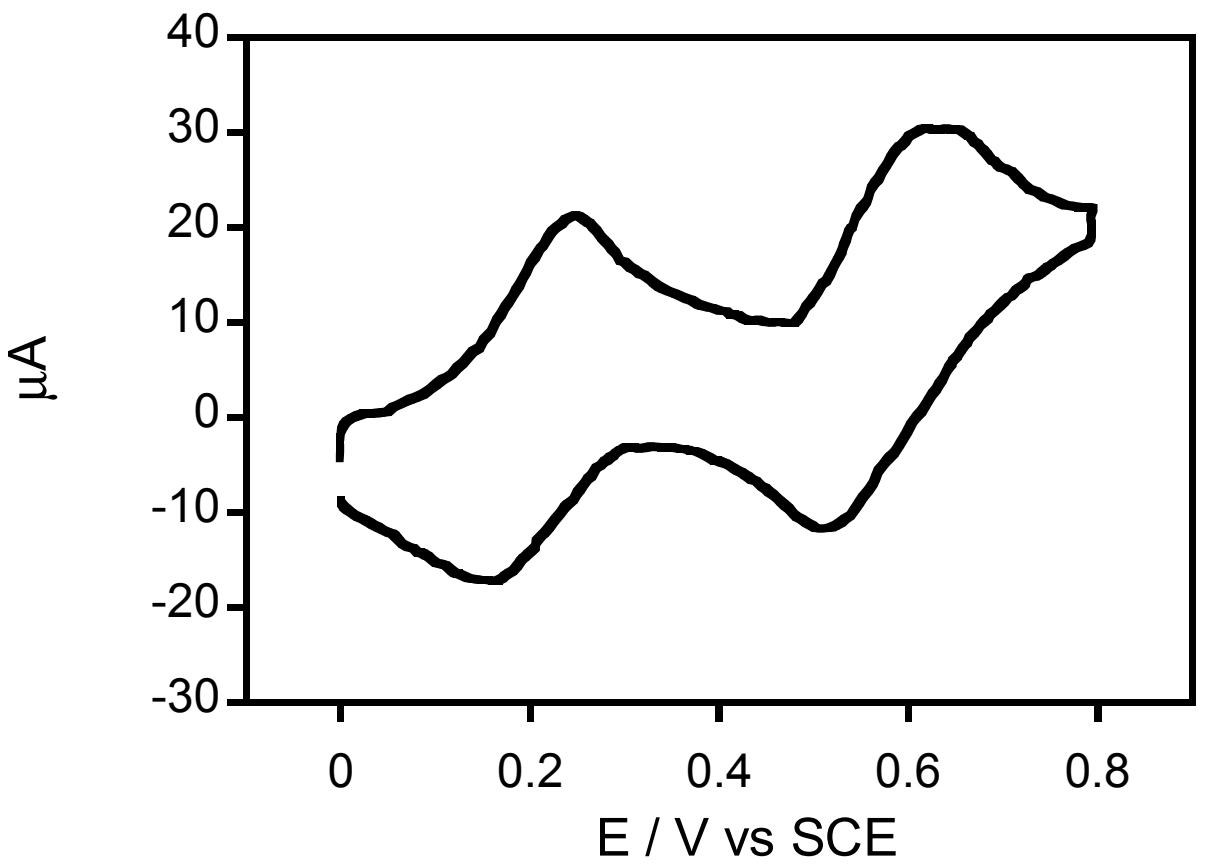

Figure 4.2.1.3. 6 Voltammogram of $\left[\mathrm{Os}(\mathrm{tpy}-\mathrm{OH})(\mathrm{bpy}) \mathrm{OH}_{2}\right]\left(\mathrm{PF}_{6}\right)_{2}$ in $0.1 \mathrm{M}$ $\mathrm{H}_{2} \mathrm{SO}_{4}+0.1 \mathrm{M} \mathrm{H}_{3} \mathrm{PO}_{4}$ aqueous solution at $\mathrm{pH} 3$ 


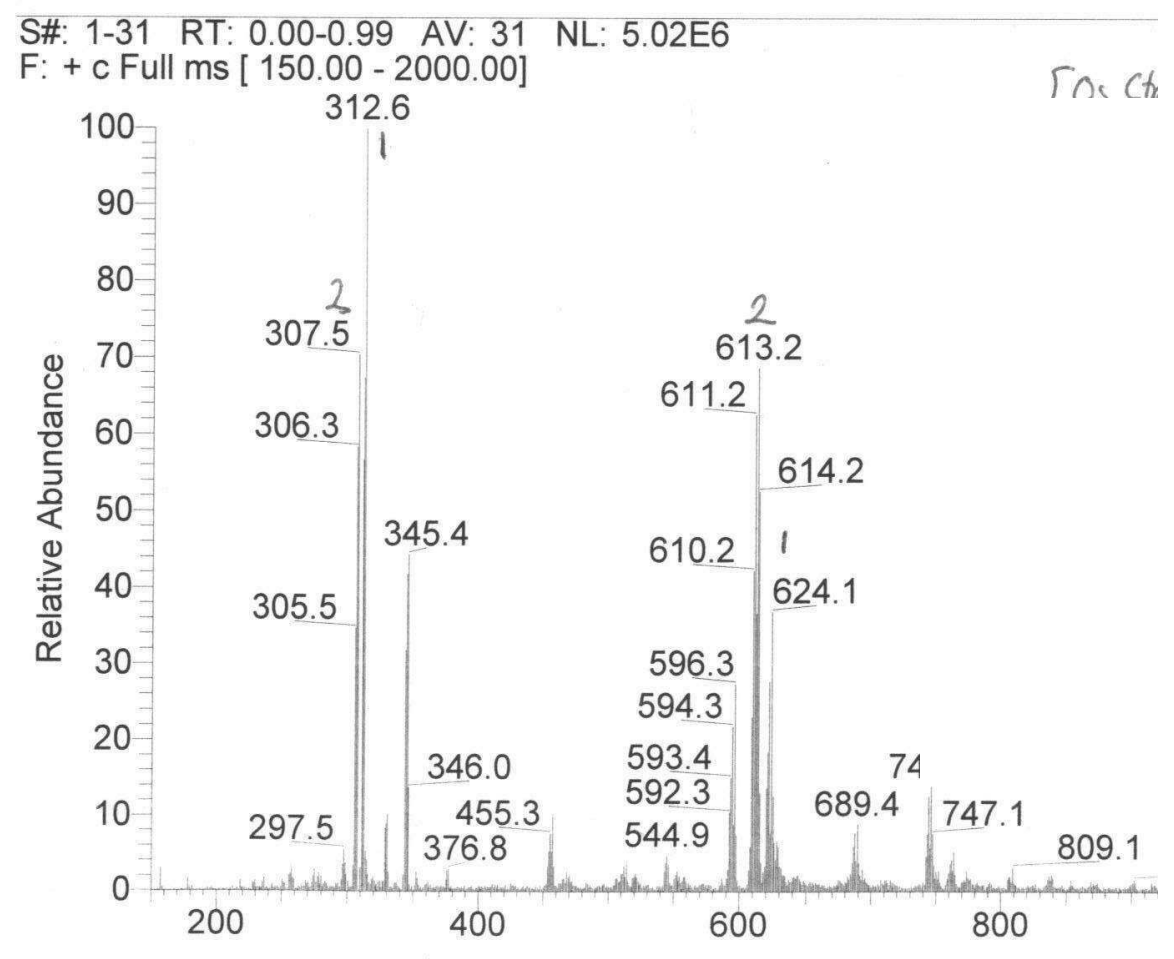

Figure 4.2.1.3. 7

Mass Spectrum of $\left[\mathrm{Os}(\right.$ tpy- $\left.-\mathrm{OH})(\mathrm{bpy}) \mathrm{OH}_{2}\right]\left(\mathrm{PF}_{6}\right)_{2}$ in $\mathrm{H}_{2} \mathrm{O}$ 


\subsubsection{Synthesis of $\left[\mathrm{Os}(\mathrm{bpy})_{2}(4-\mathrm{AMP}) \mathrm{OH}_{2}\right]\left(\mathrm{PF}_{6}\right)_{2}$}

The synthesis of $\left[\mathrm{Os}(\mathrm{bpy})_{2}(4-\mathrm{AMP}) \mathrm{OH}_{2}\right]\left(\mathrm{PF}_{6}\right)_{2}$ was achieved by either of two pathways, as seen in Figure 4.2.2.1. All DMF was used as purchased in a nitrogen covered sure-seal container, and kept refrigerated to minimize decomposition.

\subsubsection{Synthesis of $\mathrm{Os}(\mathrm{bpy})_{2} \mathrm{Cl}_{2}$}

B1 $\quad \mathrm{K}_{2} \mathrm{OsCl}_{6} \rightarrow \mathrm{Os}(\mathrm{bpy})_{2} \mathrm{Cl}_{2}$

Numerous publications are available on the synthesis of $\mathrm{Os}(\mathrm{bpy})_{2} \mathrm{Cl}_{2}{ }^{68 ; 78 ; 91 ; 92}$. All procedures are based on the original 1964 Buckingham paper ${ }^{93} .100 \mathrm{mg}$ of $\mathrm{K}_{2} \mathrm{OsCl}_{6}$ was added with 2 equivalents of bpy $(65 \mathrm{mg})$ and $2 \mathrm{ml}$ anhydrous of DMF were placed in a $50 \mathrm{ml}$ r.b.f. The solution was refluxed for 1 hour. The solution was then cooled and $20 \mathrm{ml}$ of a dilute aqueous solution of $\mathrm{Na}_{2} \mathrm{~S}_{2} \mathrm{O}_{4}$ was added in order to reduce the complex. Then it was cooled in an ice bath. The dark red/purple solid was collected, then washed with distilled, deionized water and anhydrous ether.

Typical Yield - 62\%

UV/VIS Spectrum $(\mathrm{nm})$ - Figure 4.2.2.1.1

Cyclic Voltammetry(V vs. SCE) - Figure 4.2.2.1.2 -

Single wave at $E_{1 / 2}=-0.19 \mathrm{~V}$ vs. SCE 


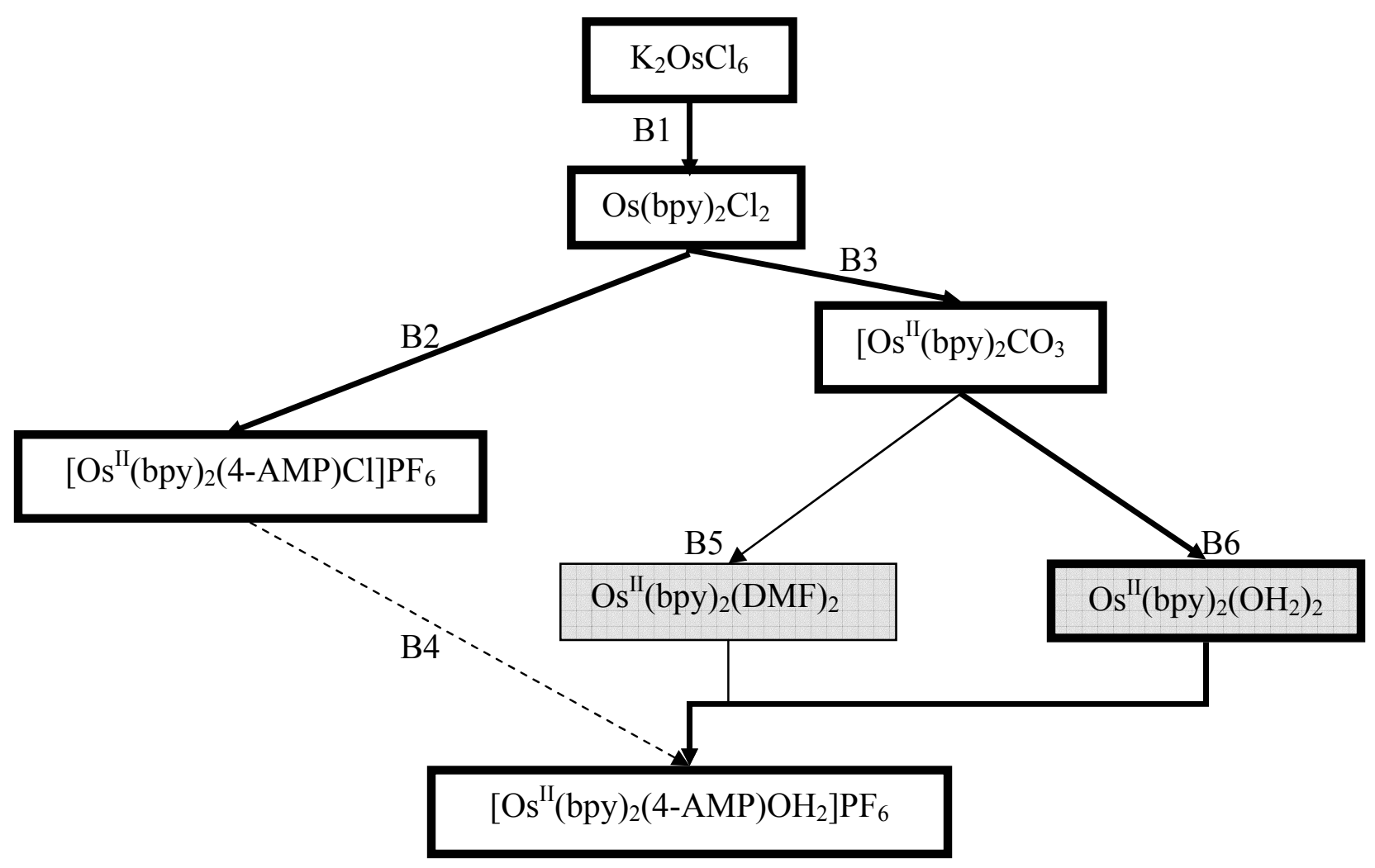

Figure 4.2.2. 1

Flowchart of [Os(bpy $\left.)_{2}(4-\mathrm{AMP}) \mathrm{OH}_{2}\right]\left(\mathrm{PF}_{6}\right)_{2}$ of Synthesis Bold arrows indicate the preferred path, while dashed arrows indicate an unsuccessful path. Shaded box represents an intermediate, not a separate product. 


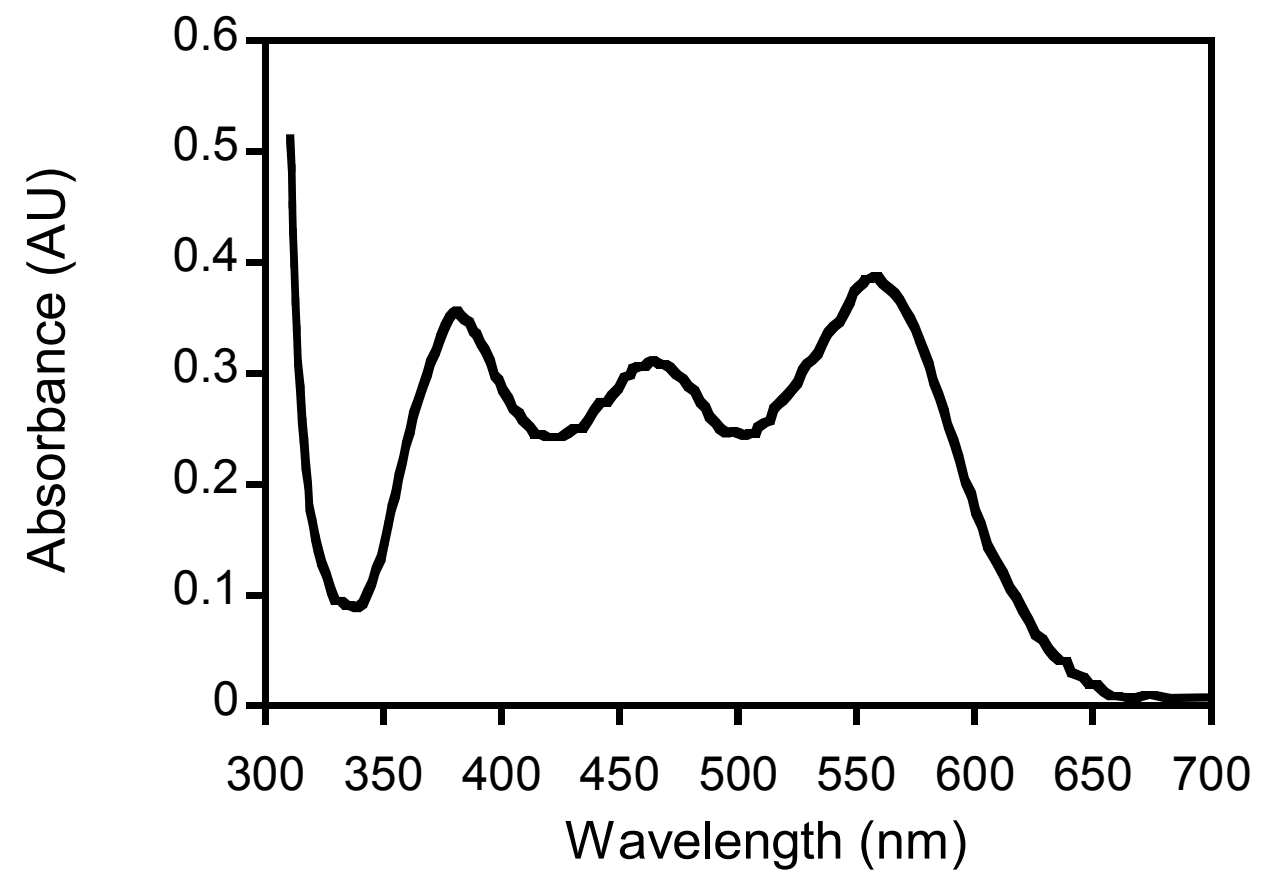

Figure 4.2.2.1. 1

UV-VIS Spectrum of Os(bpy) ${ }_{2} \mathrm{Cl}_{2}$ in $\mathrm{CH}_{3} \mathrm{CN}$ 


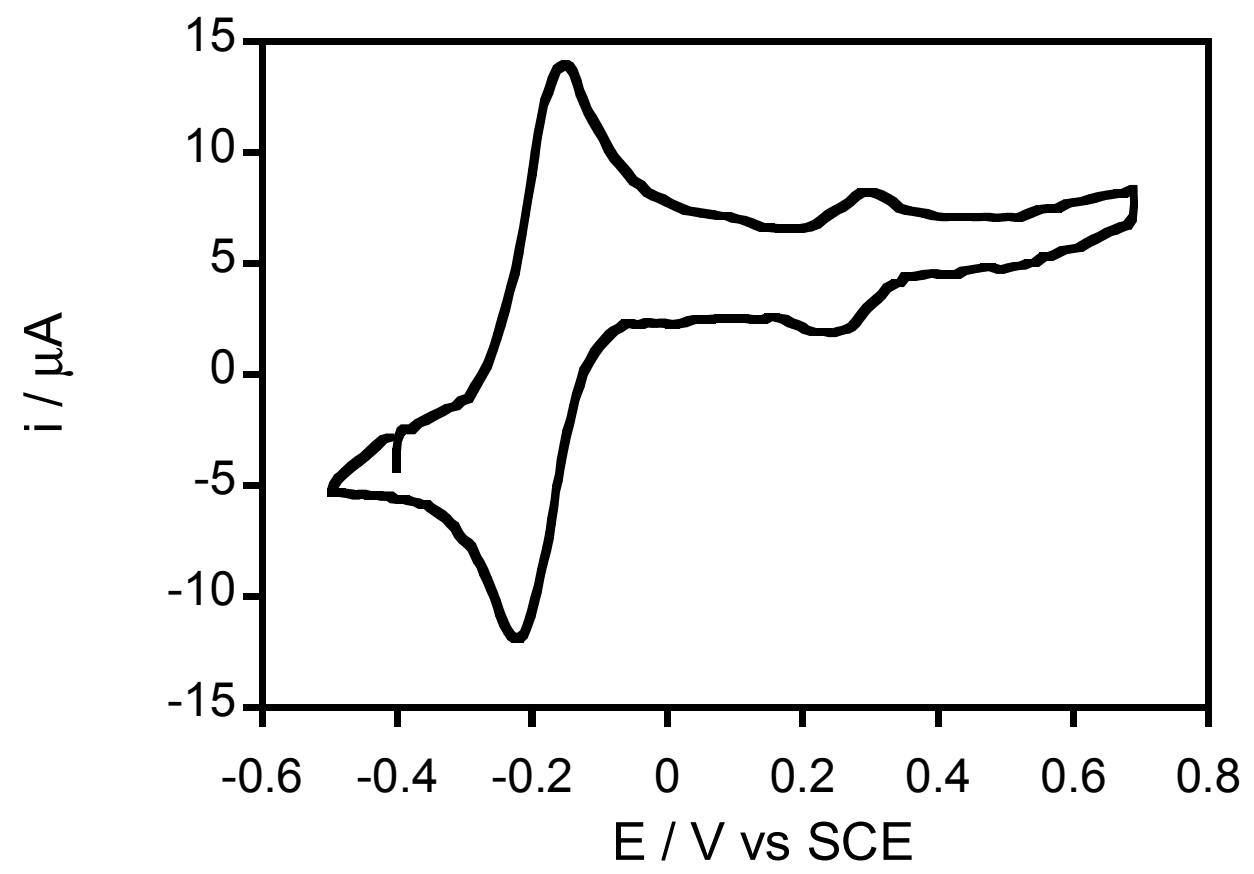

Figure 4.2.2.1. 2 Voltammogram of $\mathrm{Os}(\mathrm{bpy})_{2} \mathrm{Cl}_{2}$ in $0.1 \mathrm{M}$ tetramethylammonium tetrafluoroborate $/ \mathrm{CH}_{3} \mathrm{CN}$ 
The yield of this reaction is reasonable and the voltammogram shows a mostly pure product. Figure 4.2.2.1.1 agrees well with the literature values of 382, 467, and 562 $\mathrm{nm}^{78}$. The literature value for the formal potential, $-0.04 \mathrm{Vs} \mathrm{SSCE}^{78}$, is significantly more positive than our experimental value. The literature publication did not include the voltammogram, so visual comparison was not available. Confirmation of a pure product was achieved mainly by the success of the next step.

\subsubsection{Synthesis of $\left[\mathrm{Os}(\mathrm{bpy})_{2}(4-\mathrm{AMP}) \mathrm{Cl}\right]^{2+}$}

B2. $\mathrm{Os}(\mathrm{bpy})_{2} \mathrm{Cl}_{2} \rightarrow\left[\mathrm{Os}(\mathrm{bpy})_{2}(4-\mathrm{AMP}) \mathrm{Cl}_{\mathrm{P}} \mathrm{PF}_{6}\right.$

This step is based on the A5 synthesis step described above. $100 \mathrm{mg} \mathrm{Os}(\mathrm{bpy})_{2} \mathrm{Cl}_{2}$, one equivalent 4-AMP (18mg, $0.017 \mathrm{ml}$ ) and $2 \mathrm{ml}$ ethylene glycol were added to a $50 \mathrm{ml}$ r.b.f. The mixture was refluxed under a blanket of argon. Then it was cooled and the product precipitated by addition of $2 \mathrm{ml}$ saturated $\mathrm{NH}_{4} \mathrm{PF}_{6}$ solution. The brown solid was collected via filtration and rinsed with copious amounts of distilled, deionized water.

Typical Yield $>90 \%$

Cyclic Voltammetry $(\mathrm{V}$ vs. SCE $)$ - Figure 4.2.2.2.1. - Single wave at $\mathrm{E}_{1 / 2}=$ $+0.20 \mathrm{~V}$ vs. SCE

This synthesis is very successful. Yields were high and typically there were no impurity waves. Purification using chromatography as outlined in Section 4.2.1.1.2 was 


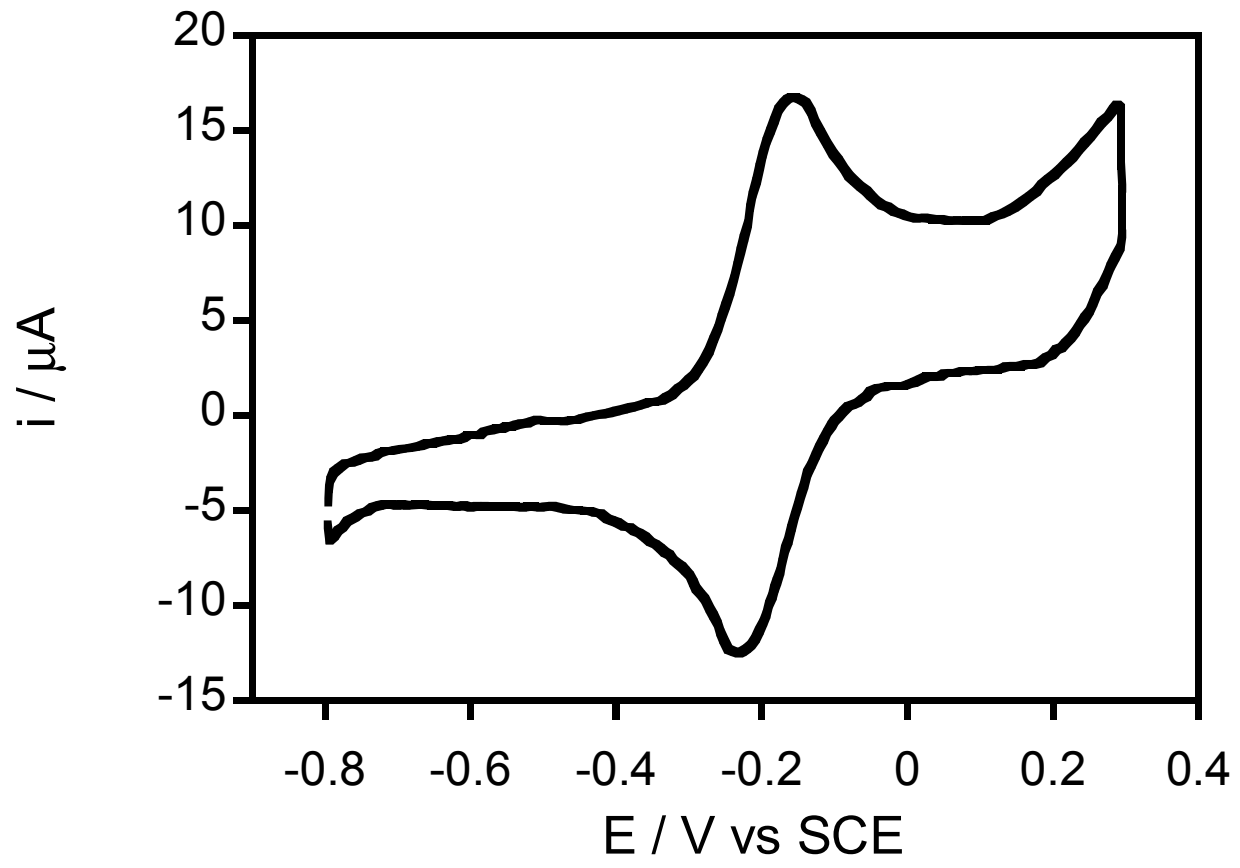

Figure 4.2.2.2. 1

$\mathrm{NaClO}_{4} / \mathrm{CH}_{3} \mathrm{CN}$

Voltammogram of [Os(bpy) $\left.{ }_{2}(4-\mathrm{AMP}) \mathrm{CI}\right]^{+}$in $0.1 \mathrm{M}$ 
successful, but largely unnecessary. All useful data was collected using unpurified product.

\subsubsection{Synthesis of $\mathrm{Os}(\mathrm{bpy})_{2} \mathrm{CO}_{3}$ and $\left[\mathrm{Os}(\mathrm{bpy})_{2}\left(\mathrm{OH}_{2}\right)_{2}\right]^{2+}$}

B3. $\mathrm{Os}(\mathrm{bpy})_{2} \mathrm{Cl}_{2} \rightarrow \mathrm{Os}(\mathrm{bpy})_{2} \mathrm{CO}_{3}$

The synthesis of $\mathrm{Os}(\mathrm{bpy})_{2} \mathrm{CO}_{3}$ from $\mathrm{Os}(\mathrm{bpy})_{2} \mathrm{Cl}_{2}$ was based on a procedure reported by Meyer ${ }^{78} .100 \mathrm{mg}$ of Os(bpy $)_{2} \mathrm{Cl}_{2}$ was added to $15 \mathrm{ml}$ of distilled, deionized water in a $50 \mathrm{ml}$ r.b.f. The solution was sparged with argon for 10 minutes to remove all oxygen. $1 \mathrm{~g}$ of sodium carbonate was added to the solution, then the solution was refluxed at $100^{\circ} \mathrm{C}$ under a blanket of argon for 2 hours. Another $1 \mathrm{~g}$ portion of sodium carbonate was added and the solution was refluxed for another two hours. A final $1 \mathrm{~g}$ portion of sodium carbonate was added and refluxed for a final 2 hours. The solution was cooled, and the black product was collected by filtration. The product was then rinsed with water that had been rendered basic by the addition of $\mathrm{NaOH}$ until the $\mathrm{pH}$ of the solution was greater than 9. The product was rinsed until the rinsings turned from black/purple to brown/red.

\section{Typical Yield - 50\%}

The yield in this reaction was moderately low. However, this is compensated by the fact that the product appears to be very pure. Analysis of the product was difficult because of the lability of the carbonate ligand. Upon dissolution in any acidic aqueous medium, the carbonate was immediately displaced by aqua groups, forming 
$\left[\mathrm{Os}(\mathrm{bpy})_{2}\left(\mathrm{OH}_{2}\right)_{2}\right]^{2+}$. This conversion is quantitative. The success of the reaction was determined by the purity of the bis-aqua species, which was readily seen in the UV-VIS spectrum and voltammogram of $\left[\mathrm{Os}(\mathrm{bpy})_{2}\left(\mathrm{OH}_{2}\right)_{2}\right]^{2+}$. Figure 4.2.2.3.1 was the voltammogram of $\left[\mathrm{Os}(\mathrm{bpy})_{2}\left(\mathrm{OH}_{2}\right)_{2}\right]^{2+}$ taken from the literature ${ }^{65}$.

\section{Cyclic Voltammetry(V vs. SCE) - Figure 4.2.2.3.2}

$$
\begin{aligned}
& E_{1 / 2}(\mathrm{pH} 1.5)=+0.06(2+/ 3+),+0.54(3+/ 5+), \\
& +0.72(5+/ 6+)
\end{aligned}
$$

\section{Coulometry $-95 \%$ pure}

Both the UV-VIS spectrum and the voltammogram matched the literature data. Also the coulometry suggests that the product was highly pure.

\subsubsection{Synthesis of $\left[\mathrm{Os}(\mathrm{bpy})_{2}(4-\mathrm{AMP}) \mathrm{OH}_{2}\right]^{2+}$}

B4. $\left[\mathrm{Os}(\mathrm{bpy})_{2}(4-\mathrm{AMP}) \mathrm{Cl}\right] \mathrm{PF}_{6} \rightarrow\left[\mathrm{Os}(\mathrm{bpy})_{2}\left(4-\mathrm{AMP}_{6}\right) \mathrm{OH}_{2}\right]\left(\mathrm{PF}_{6}\right)_{2}$

This synthesis is based on the A6 step. $100 \mathrm{mg}$ of $\left[\mathrm{Os}(\mathrm{bpy})_{2}(4-\mathrm{AMP}) \mathrm{CI}_{\mathrm{P}} \mathrm{F}_{6}\right.$ was added to $3 \mathrm{ml}$ of triflic acid in a $50 \mathrm{ml}$ r.b.f., and kept under a blanket of argon. The mixture was heated to $110^{\circ} \mathrm{C}$ in an oil bath for 5 hours. After 5 hours, the condenser was removed, and most of the triflic acid was evaporated. $5 \mathrm{ml}$ of distilled, deionized water was added, along with a small amount of Zinc amalgam. The solution was stirred for 3 hours. The amalgam was filtered off, and the product precipitated with the addition of $\mathrm{NH}_{4} \mathrm{PF}_{6}$. The product was rinsed thoroughly with distilled, deionized water. 

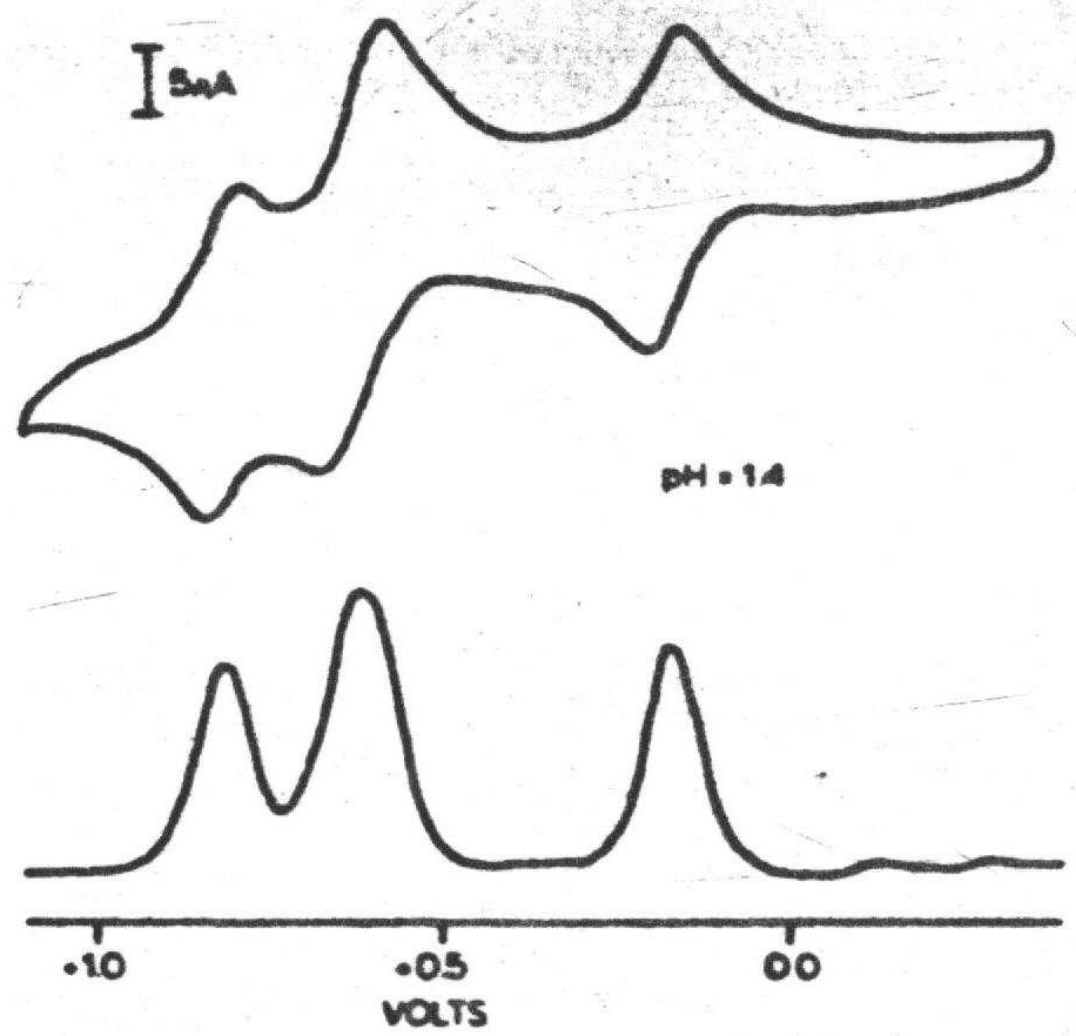

Figure 4.2.2.3. $1 \quad$ Voltammogram of $\left[\mathrm{Os}(\mathrm{bpy})_{2} \mathrm{OH}_{2}\right]_{2}{ }^{2+}$ from the Literature. Note that this figure uses the American axis naming convention, not the IUPAC convention used by the Finklea group 


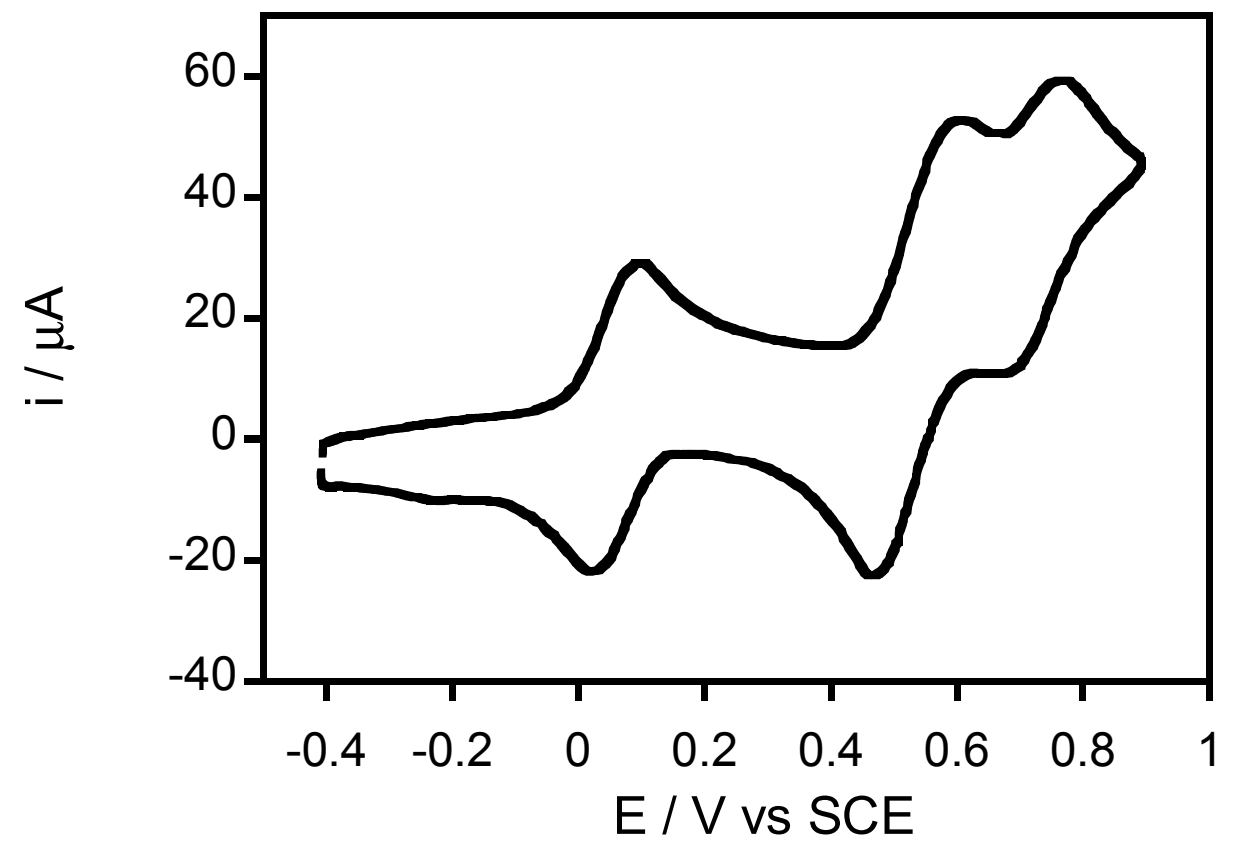

Figure 4.2.2.3. 2 Voltammogram of $\left[\mathrm{Os}(\mathrm{bpy})_{2} \mathrm{OH}_{2}\right]_{2}{ }^{2+}$ in $0.1 \mathrm{M} \mathrm{HClO}_{4}$ in aqueous solution, $\mathrm{pH} 1.08$. 


\section{Cyclic Voltammetry(V vs. SCE $)-E_{1 / 2}(p H 1.0)=0.16,0.28,0.48$ \\ $E_{1 / 2}(p H ~ 8.0)=0.0,0.16,0.45$}

The product is quite impure. There is a $\mathrm{pH}$ dependence with one of the waves, however this was masked by the impurities present.

B5. Os $(\text { bpy })_{2} \mathrm{CO}_{3} \rightarrow\left[\mathrm{Os}(\mathrm{bpy})_{2}(\mathrm{~L})_{2}\right]\left(\mathrm{PF}_{6}\right)_{2} \rightarrow\left[\mathrm{Os}(\mathrm{bpy})_{2}(4-\mathrm{AMP}) \mathrm{OH}_{2}\right]\left(\mathrm{PF}_{6}\right)_{2}$

This approach is based upon the 1988 publication by Meyer ${ }^{78}$. Meyer's approach allows for the substitution of two ligands onto the metal center by displacement of the carbonate ligand with two equivalents of the solvent. Both DMF and t-butylalcohol were used as solvents. Since the solvent is fairly labile, the two solvent ligands are readily displaced by any less labile ligands that are introduced. In the Meyer synthesis, the first solvent ligand is displaced with a 27 fold excess addition of the first desired ligand, followed by 1 hour of reflux. Substitution of the second ligand occurred only under more vigorous conditions. A 600 fold excess of the second ligand was added followed by another hour of reflux. This procedure was modified in our synthesis. $100 \mathrm{mg}$ of $\mathrm{Os}(\mathrm{bpy})_{2} \mathrm{CO}_{3}$ was combined with $60 \mathrm{ml}$ t-butylalcohol in a $100 \mathrm{ml}$ r.b.f. The solution was sparged with argon for 10 minutes. 8 drops of $\mathrm{HPF}_{6}$ was added under a blanked of argon. A 27 fold excess of 4-AMP was added to the solution. The mixture was refluxed for 1 hour. The solution was cooled and the majority of the solvent was removed via the rotovap. A black precipitate had formed (product $A$ ), and was removed from the solution via filtration. $10 \mathrm{ml}$ of distilled, deionized water was added, then the product was precipitated with $\mathrm{NH}_{4} \mathrm{PF}_{6}$ (product $\mathrm{B}$ ). It was hoped that because 
of the rigourous conditions necessary for the second ligand substitution, the reaction would stop after the $1^{\text {st }}$ substitution. The labile solvent should then be displaced by water upon its addition.

\section{Cyclic Voltammetry(V vs. SCE) - \\ - Product $A-E_{1 / 2}(p H 2)=-0.15, E_{1 / 2}(p H ~ 8)=-0.12$ \\ - Product B - multiple overlapping waves}

Product $A$ only showed one electroactive wave, with an $\mathrm{E}_{1 / 2} \approx-0.15 \mathrm{~V}$ vs. SCE, at all pH's. The $\mathrm{pH}$ independence suggests that it is not $\left[\mathrm{Os}(\mathrm{bpy})_{2}(4-\mathrm{AMP}) \mathrm{OH}_{2}\right]\left(\mathrm{PF}_{6}\right)_{2}$. Product B is very dirty, with at least 4 different electroactive waves. Two of these waves do appear to shift with $\mathrm{pH}$, however, the synthesis is too dirty to be certain. B6. $\mathrm{Os}(\mathrm{bpy})_{2} \mathrm{CO}_{3} \rightarrow\left[\mathrm{Os}(\mathrm{bpy})_{2}\left(\mathrm{OH}_{2}\right)_{2}\right]\left(\mathrm{PF}_{6}\right)_{2} \rightarrow\left[\mathrm{Os}(\mathrm{bpy})_{2}(4-\mathrm{AMP}) \mathrm{OH}_{2}\right]\left(\mathrm{PF}_{6}\right)_{2}$ To our knowledge, this synthesis pathway has not been reported before. Synthesis pathway B4 is based on the displacement of a labile solvent ligand with the 4-AMP ligand. Meyer used DMF and t-butylalcohol because the substituted ligands would be bulky and labile It was possible that water, a smaller, less steric ligand, could also be displaced by 4-AMP under refluxing conditions. $100 \mathrm{mg} \mathrm{Os}(\mathrm{bpy})_{2} \mathrm{CO}_{3}$ was added to 50 $\mathrm{ml}$ distilled, deionized water in a $100 \mathrm{ml}$ r.b.f. The solution was sparged with argon for 10 minutes. 5 drops of 4-AMP (a large excess) was added, then the solution was refluxed for 4 hours. The solution was cooled, and the product was precipitated with the addition of $\mathrm{NH}_{4} \mathrm{PF}_{6}$. The product was washed with distilled, deionized water, then dried in a dessicator.

Typical Yield - $70 \%$ 
Cyclic Voltammetry (V vs. SCE) - Figure 4.2.2.4.1

$$
\begin{aligned}
& E_{1 / 2}(p H ~ 2.85)=+0.18 \\
& E_{1 / 2}(p H 5.40)=+0.04
\end{aligned}
$$

Coulometry $-84 \%$ pure

The yield of this synthesis was reasonable, and voltammetry showed that the product did not require further purification. This was confirmed by coulometry, which showed that the product was $84 \%$ electroactively pure. Upon initial examination, the material showed the proper nernstian $\mathrm{pH}$ dependence. 


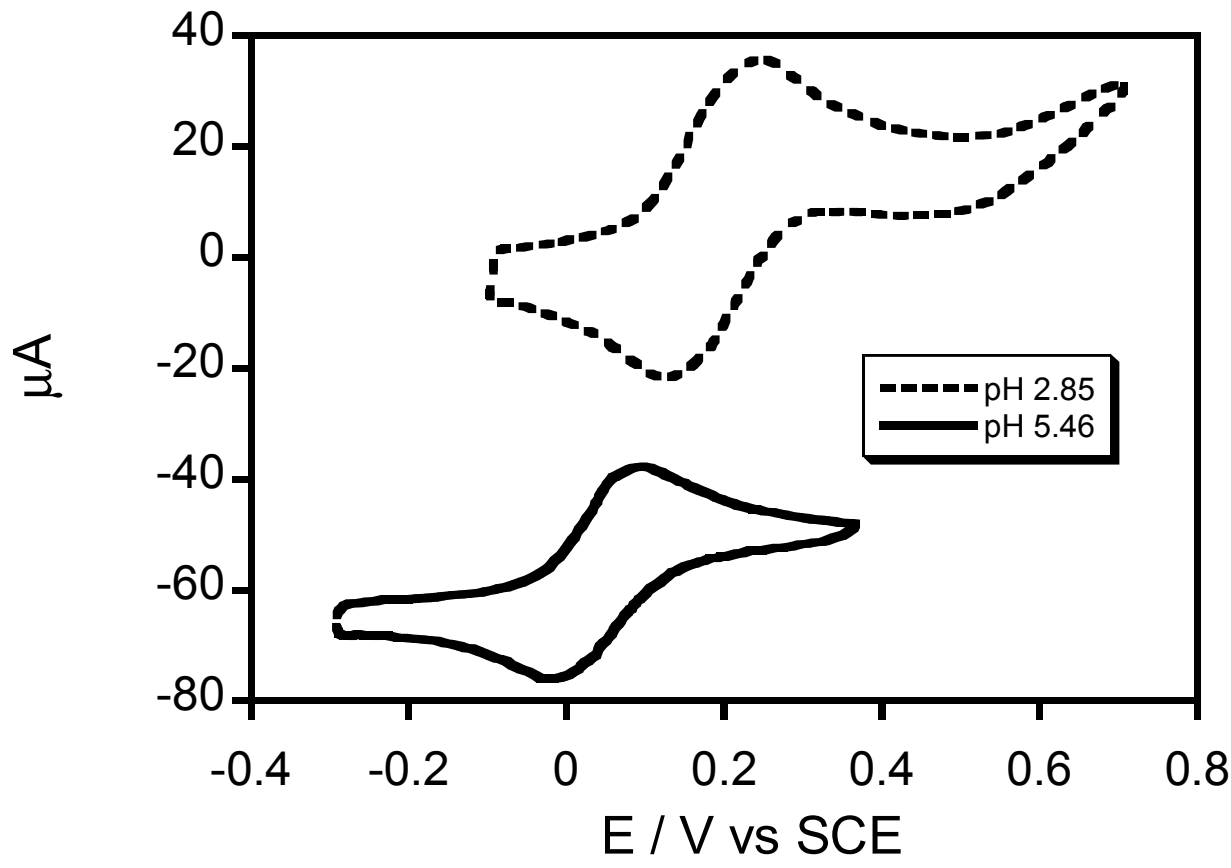

Figure 4.2.2.4. $1 \quad$ Voltammograms of $\left[\mathrm{Os}(\mathrm{bpy})_{2}(4-\mathrm{AMP}) \mathrm{OH}\right]_{2}{ }^{2+}$ in $0.5 \mathrm{M}$ $\mathrm{K}_{2} \mathrm{SO}_{4}+0.1 \mathrm{M}$ Britton-Robinson buffer in aqueous solution - Method B6 


\subsubsection{Preparation and Deposition of Monolayers}

The formation of a redox center attached to a SAM occurred in two steps. First, the monolayer was deposited onto the surface of a gold electrode. Then the redox center was coupled to the monolayer through the formation of an amide bond or ester

bond. A general discussion on monolayer deposition is given in Chapter 2, and will not be repeated here. In the osmium project, two distinct monolayers were used. A uniform monolayer of 16-mercaptohexadecanoic acid, $\mathrm{HS}\left(\mathrm{CH}_{2}\right){ }_{15} \mathrm{COOH}$, was used in the analysis of $\left[\mathrm{Os}(\mathrm{tpy}-\mathrm{OH})(\mathrm{bpy}) \mathrm{Cl}_{\mathrm{PF}} \mathrm{F}_{6}\right.$ and $\left[\mathrm{Os}(\mathrm{bpy})_{2}(4-\mathrm{AMP}) \mathrm{Cl}\right] \mathrm{PF} 6$. Gold electrodes were immersed overnight in $20 \mathrm{ml}$ of ethanol that contained a small amount $(<5 \mathrm{mg})$ of the thiol. In the analysis of $\left[\mathrm{Os}(\mathrm{bpy})_{2}(4-\mathrm{AMP}) \mathrm{OH}_{2}\right]\left(\mathrm{PF}_{6}\right)_{2}$, a mixed monolayer was used. $5 \mathrm{mg}$ of 12-mercaptododecanol , $\mathrm{HS}\left(\mathrm{CH}_{2}\right){ }_{12} \mathrm{OH}$, was added to $20 \mathrm{ml}$ ethanol in a small vial, and $5 \mathrm{mg} \mathrm{HS}\left(\mathrm{CH}_{2}\right){ }_{15} \mathrm{COOH}$ was added to a separate vial conaining $20 \mathrm{ml}$ ethanol. The deposition solution was created with the addition of 10 drops from each vial to 20 $\mathrm{ml}$ of ethanol $\left(50 / 50 \mathrm{HS}\left(\mathrm{CH}_{2}\right){ }_{15} \mathrm{COOH} / \mathrm{HS}\left(\mathrm{CH}_{2}\right){ }_{12} \mathrm{OH}\right)$. All electrodes were cleaned as described in Chapter 2, prior to overnight deposition.

\subsubsection{Coupling of Redox Centers to Monolayers}

The osmium redox centers were attached to monolayers using two different coupling procedures. Both procedures are based on a carbodiimide coupling reaction. 
The carbodiimide coupling reaction is a common technique for reacting a carboxylic acid with either a primary alcohol or amine in the formation of an ester or amide, respectively ${ }^{94}$. This reaction has also had success in the formation of ether linkages from alcohols ${ }^{95}$. Two carbodiimides are typically used: N,N'-dicyclohexyl-carbodiimide (DCC) and 1-ethyl-3(3-dimethylaminopropyl)-carbodiimide (EDC). DCC is organic soluble carbodiimide, and EDC is used in aqueous coupling reactions. In the context of this research project, the carbodiimide coupling reaction was used to attach the osmium redox couples to the carboxylic acid terminated monolayer via amide or ester linkage formation.

In this project, the DCC coupling reaction was performed in accordance to the literature $^{96} .824 \mathrm{mg}$ of DCC was added to $58 \mathrm{mg}$ DMAP and $10 \mathrm{ml}$ methylene chloride in a $20 \mathrm{ml}$ glass vial. About $5 \mathrm{mg}$ of the osmium redox couple was added to the vial and any insolubles were filtered off. The solution was then sparged with argon for 10 minutes. The monolayer coated electrode was rinsed thoroughly with methylene chloride, then placed in the coupling solution. Deposition times varied, but were typically around 1 hour followed by 1 hour of annealing in an ethanol solution containing $\mathrm{HS}\left(\mathrm{CH}_{2}\right)_{15} \mathrm{COOH}$. This method was used to attach both [Os(tpy$\mathrm{OH})(\mathrm{bpy}) \mathrm{Cl}_{\mathrm{PF}} \mathrm{F}_{6}$ and $\left[\mathrm{Os}(\mathrm{bpy})_{2}(4-\mathrm{AMP}) \mathrm{Cl}_{3} \mathrm{PF}_{6}\right.$ to their respective monolayers.

The EDC reaction was based on a method provided by Dr. Finklea. Two different final procedures were used. For the coupling of $\left[\mathrm{Os}(\mathrm{bpy})_{2}(4-\mathrm{AMP}) \mathrm{Cl}_{\mathrm{P}}\right] \mathrm{F}_{6}$, $150 \mathrm{mg}$ of EDC was added to a vial containing $150 \mathrm{mg} \mathrm{KNO}_{3}$ and $20 \mathrm{ml}$ of $0.005 \mathrm{M}$ $\mathrm{H}_{3} \mathrm{PO}_{4}$, whose $\mathrm{pH}$ had been adjusted to 7 using $\mathrm{NaOH}$. Around 2-3 mg of osmium 
product was added to the vial. Any insolubles were filtered, then the solution was sparged with argon for 10 minutes. The monolayer coated electrode was rinsed with distilled, deionized water, then deposited into the coupling solution. After 1 hour in the coupling solution, the electrode was annealed in an ethanol solution containing $\mathrm{HS}\left(\mathrm{CH}_{2}\right)_{15} \mathrm{COOH}$ for 1 hour. In the case of $\left[\mathrm{Os}(\mathrm{bpy})_{2}(4-\mathrm{AMP}) \mathrm{OH}_{2}\right]\left(\mathrm{PF}_{6}\right)_{2}$, the deposition conditions had to be modified in order to achieve good monolayer coupling. $15 \mathrm{mg}$ of EDC was added to $20 \mathrm{ml}$ of $.1 \mathrm{M} \mathrm{H}_{3} \mathrm{PO}_{4}$ at $\mathrm{pH} 7$ in a vial. $2-3 \mathrm{mg}$ of osmium product was added to the vial and any insolubles were removed. After 10 minutes of sparging in argon, a freshly rinsed monolayer coated electrode was placed in the vial. After 15 minutes in the coupling solution, the electrode was removed, rinsed thoroughly and covered in distilled, deionized water, until use. The amount of EDC needed to be lowered in this reaction. Reactions with $150 \mathrm{mg}$ EDC formed monolayers with a pH independent impurity wave slightly negative of the expected wave. By shortening the deposition time and reducing the amount of EDC used, the impurity wave disappeared. It was thought that derivatized urea side product was interacting with, or displacing the aqua group causing the impurity wave.

\subsection{Results - The Osmium System}

\subsubsection{Results - Introduction}

While the ultimate goal is to explore the thermodynamics and kinetics of the $\left[\mathrm{Os}(\mathrm{L})_{5} \mathrm{OH}_{2}\right]^{2+}$ system, it is also of interest to study some of the precursor products, 
specifically the $\left[\mathrm{Os}(\mathrm{L})_{5} \mathrm{Cl}\right]^{+}$system. A number of publications, mostly by Forster and Abruna ${ }^{12 ; 40 ; 69 ; 97 ; 98}$, have dealt with the thermodynamics and kinetics of this system. It was of interest to compare the results of the $[\mathrm{Os}(\mathrm{tpy}-\mathrm{OH})(\mathrm{bpy}) \mathrm{Cl}] \mathrm{PF} \mathrm{F}_{6}$ and $\left[\mathrm{Os}(\mathrm{bpy})_{2}(4-\right.$ $\mathrm{AMP}) \mathrm{Cl}_{\mathrm{PF}}$ systems to similar systems in the literature. It was also beneficial to explore these systems in order to have a better background in exploring the aqua system.

\subsubsection{Results - The [Os(tpy-OH)(bpy)Cl] ${ }^{+}$System}

The synthesis of [Os(tpy-OH)(bpy)Cl]PF 6 was fairly straightforward. As described above, alumina chromatography using acetone/acetonitrile/methanol as the progressive eluent was successful in purifying the compound. However, it is important to note that purification was not necessary in most batches. In fact, all significant data was obtained with product that had not been purified. Proper synthesis technique removed the necessity of purification. [Os(tpy-OH)(bpy)Cl] ${ }^{+}$readily coupled to monolayers using the DCC coupling reaction. Figure 4.3.2.1 was a typical voltammogram of the $[\mathrm{Os}(\mathrm{tpy}-\mathrm{OH})(\mathrm{bpy}) \mathrm{Cl}]^{+}$complex attached to a SAM. The reversible formal potential was $E_{1 / 2}=+0.26 \mathrm{~V}$ vs. SCE. Typical reversible peak splittings, $\Delta E_{p}$, were less than $15 \mathrm{mV}$. Typical FWHM values were about $120 \mathrm{mV}$. These near-ideal values suggest that there is little thermodynamic heterogeneity. The redox couple was quite stable over time. A scan rate study was performed in $0.1 \mathrm{M} \mathrm{H}_{2} \mathrm{SO}_{4}+0.1 \mathrm{M} \mathrm{H}_{3} \mathrm{PO}_{4}$. Figure 4.3.2.2 shows the effect that the scan rate had on the voltammogram. As can 


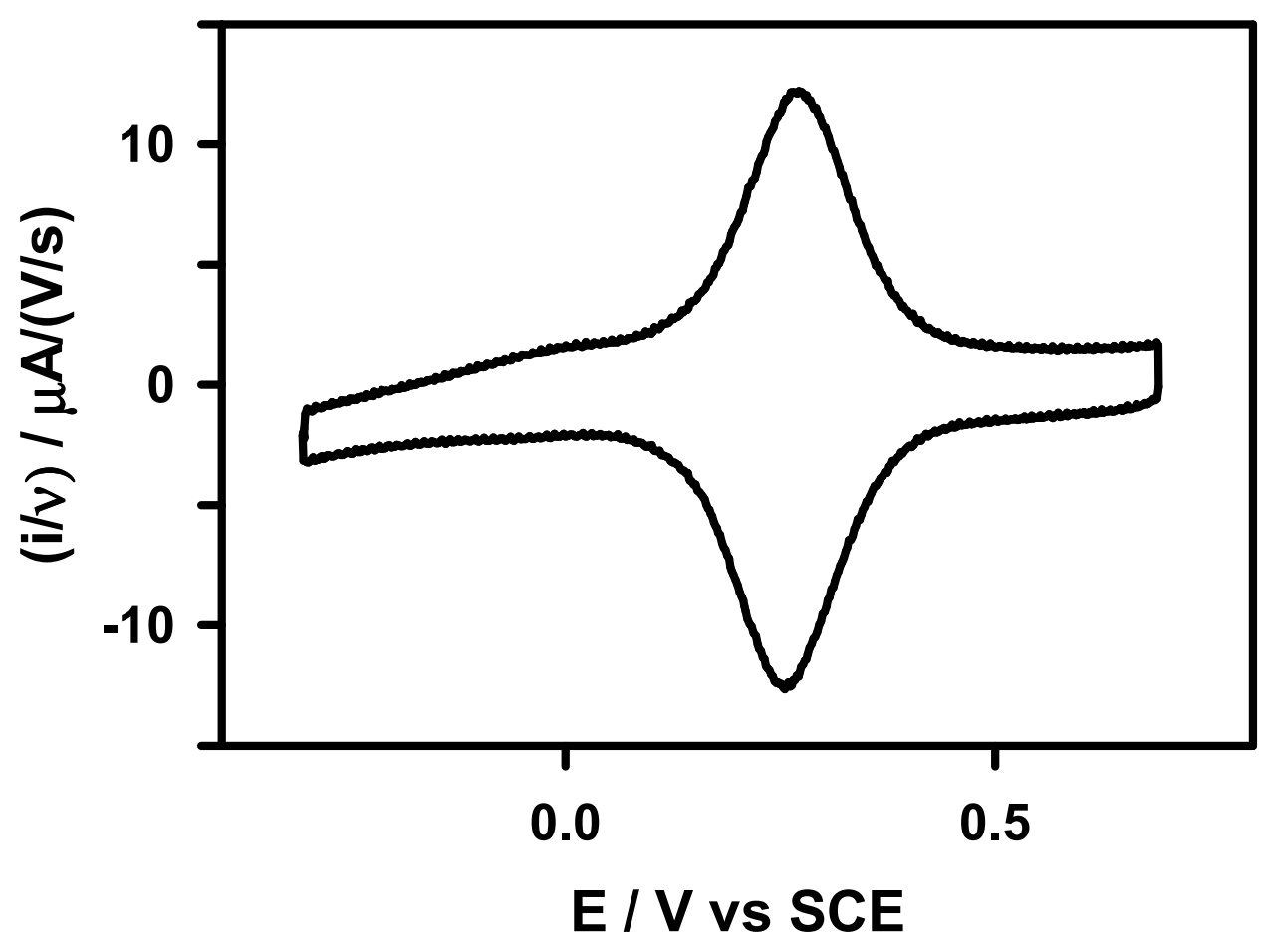

Figure 4.3.2. 1 Voltammogram of [Os(tpy-OH)(bpy)Cl] $]^{+}$attached to $\mathrm{HS}\left(\mathrm{CH}_{2}\right)_{15} \mathrm{COOH}$ Monolayer on a Gold Electrode in $0.1 \mathrm{M} \mathrm{H}_{2} \mathrm{SO}_{4}+0.1 \mathrm{M} \mathrm{H}_{3} \mathrm{PO}_{4}$ aqueous solution 


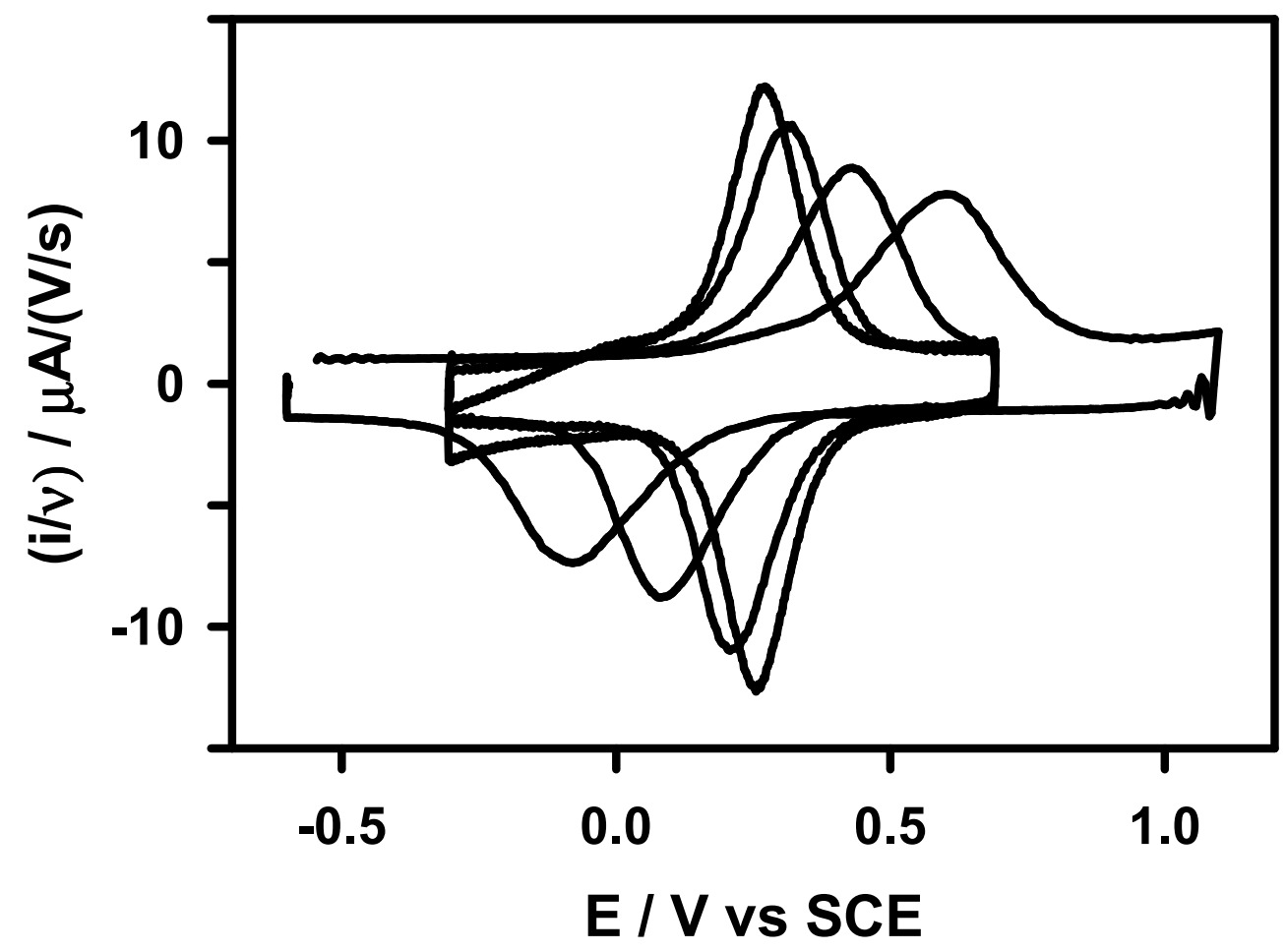

Figure 4.3.2. $2 \quad$ Voltammograms of [Os(tpy-OH)(bpy)Cl] ${ }^{+}$at multiple Scan Rates in $0.1 \mathrm{M} \mathrm{H}_{2} \mathrm{SO}_{4}+0.1 \mathrm{M} \mathrm{H}_{3} \mathrm{PO}_{4}$ aqueous solution. Scan rates are 0.1, 1, 10 , and $100 \mathrm{~V} / \mathrm{s}$. Currents are normalized with respect to the scan rate. 
be seen from the figure, at high scan rates the anodic and cathodic waves stayed symmetrical. The standard rate constant, $\mathrm{k}_{\mathrm{s}}$, was extracted using Tafel analysis. Tafel plots were created at $80 \%, 50 \%$, and $20 \%$ conversion, as is seen in Figure 4.3.2.3. $\mathrm{k}_{\mathrm{s}}$ values are listed in Table 4.3.2.1.

\subsubsection{Results - The $\left[\mathrm{Os}(\mathrm{bpy})_{2}(4-\mathrm{AMP}) \mathrm{Cl}\right]^{+}$System}

The $\left[\mathrm{Os}(\mathrm{bpy})_{2}(4-\mathrm{AMP}) \mathrm{Cl}\right]^{+}$redox couple readily attached to the $\mathrm{HS}\left(\mathrm{CH}_{2}\right){ }_{15} \mathrm{COOH}$ monolayer using either the EDC or DCC coupling method. Figure

4.3.3.1 is indicative of a typical voltammogram. Under thermodynamically reversible conditions, the redox couple exhibits near-ideal behavior, with peak splittings of around $12-15 \mathrm{mV}$, and FWHM of around $120 \mathrm{mV}$. Scan Rate studies were performed over a pH range of 1.5 to 11 to explore the dependence of the redox couple on $\mathrm{pH}$. Figure 4.3.3.2 shows the typical effect of scan rate on the voltammogram. Figure 4.3.3.3 and Figure 4.3.3.4 show the absence of $\mathrm{pH}$ efffects on the peak shapes and peak positions under both thermodynamic and kinetic control, respectively. Note that peak potentials at low and high scan rate do not show a dependence on $\mathrm{pH}$. Table 4.3.3.1 shows the effect of $\mathrm{pH}$ on the standard rate constant. 


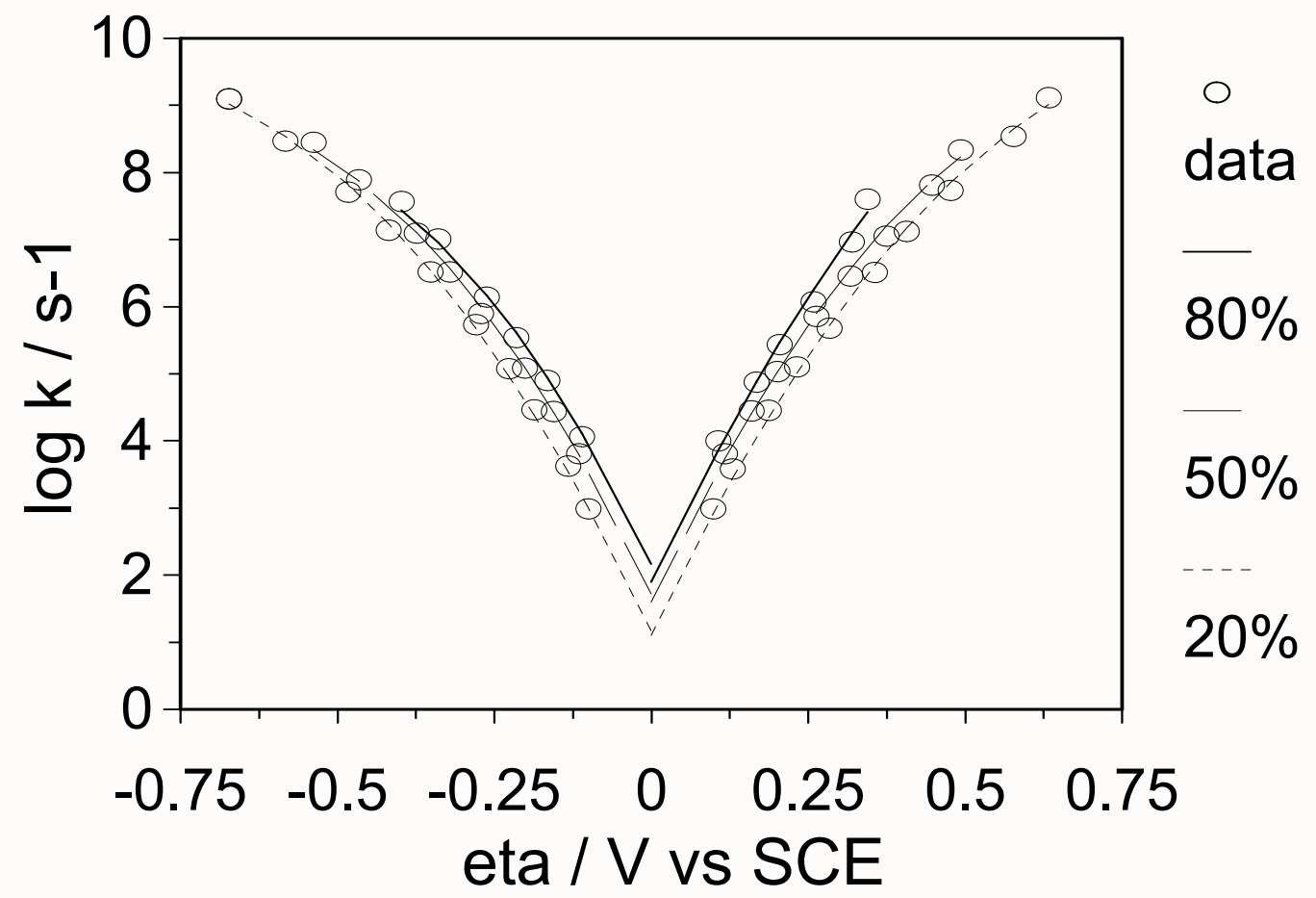

Figure 4.3.2. 3

Tafel Plots of [Os(tpy-OH)(bpy)Cl] 


\begin{tabular}{|l|l|l||}
\hline WAVE & STANDARD RATE & REORGANIZATION \\
CONSTANT & ENERGY \\
\hline 80 -anodic & 6.7 & 0.85 \\
\hline 80 -cathodic & 8.8 & 0.50 \\
\hline 50 -anodic & 5.0 & 0.70 \\
\hline 50 -cathodic & 5.6 & 0.60 \\
\hline 20-anodic & 3.0 & 0.75 \\
\hline 20-cathodic & 3.2 & 0.70 \\
\hline
\end{tabular}

\begin{tabular}{|c|c|}
\hline AVERAGE $\lambda$ & AVERAGE $\mathbf{k}_{\mathbf{s}}$ \\
\hline \hline $0.68 \pm 0.12 \mathrm{eV}$ & $5 \pm 2 \mathrm{~s}^{-1}$ \\
\hline
\end{tabular}




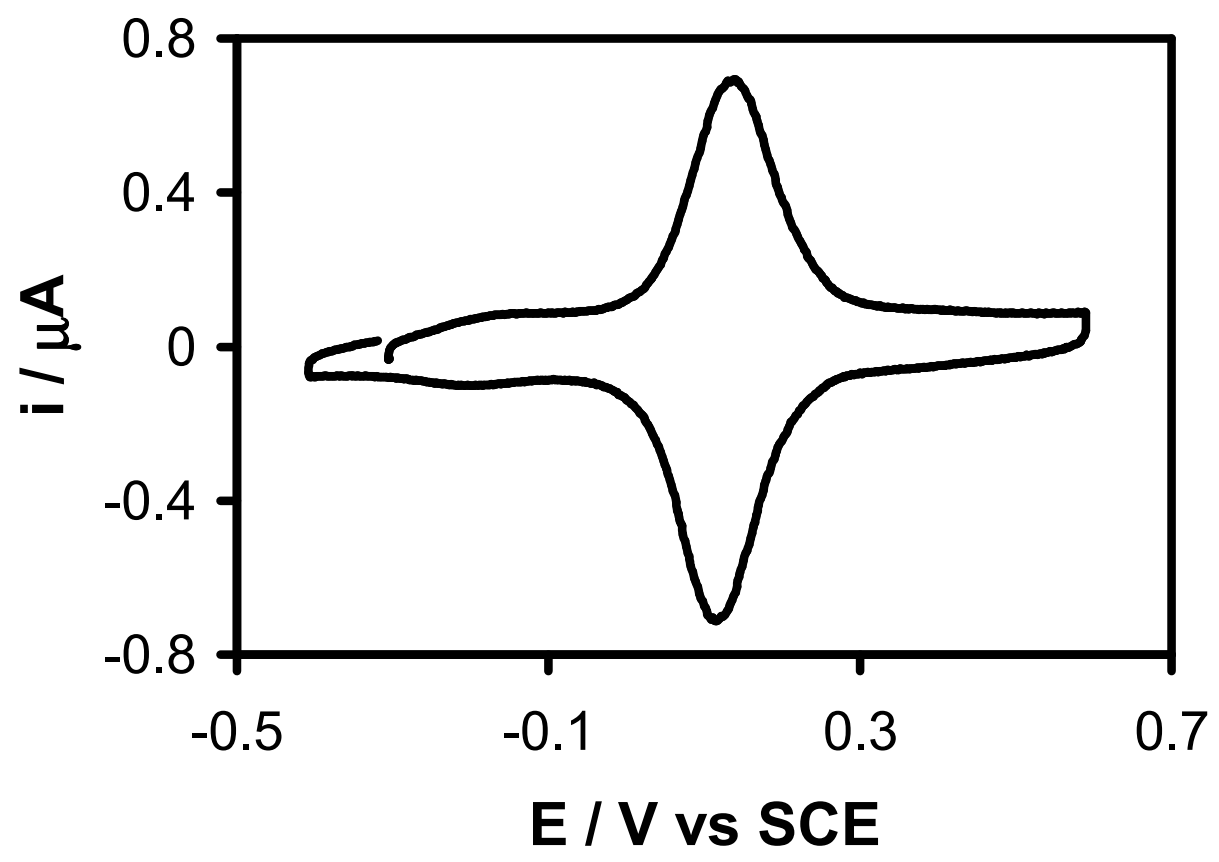

Figure 4.3.3. 1

Voltammogram of [Os(bpy $)_{2}$ (4-AMP)Cl] ${ }^{+}$attached to $\mathrm{HS}\left(\mathrm{CH}_{2}\right){ }_{15} \mathrm{COOH}$ Monolayer on a Gold Electrode in $1.0 \mathrm{M} \mathrm{NaClO}_{4}$ 


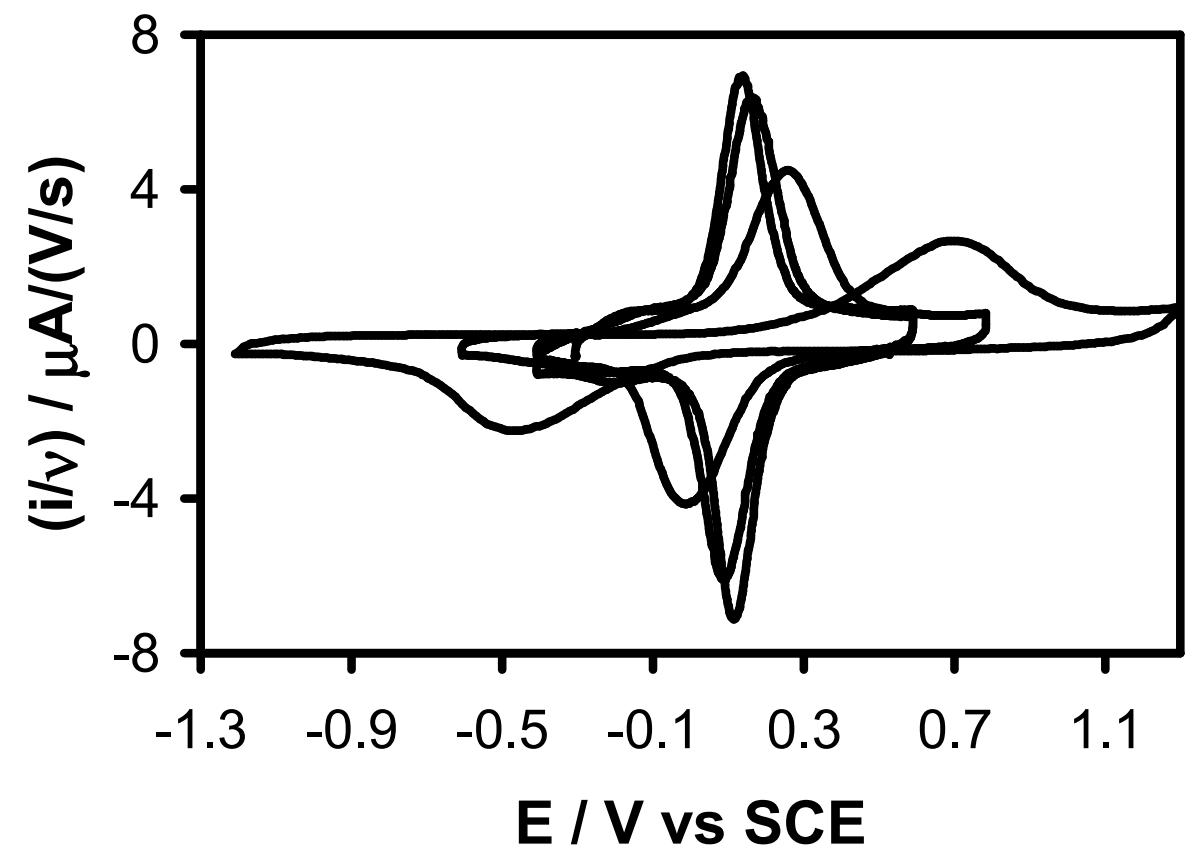

Figure 4.3.3. 2 Voltammograms of [Os(bpy)2(4-AMP)Cl] in $1.0 \mathrm{M}$ $\mathrm{NaClO}_{4}$, at multiple scan rates. Scan rates are $0.1,1,10,100$, and $1000 \mathrm{~V} / \mathrm{s}$. Currents are normalized with respect to the scan rate. $\mathrm{pH}$ was adjusted to $\mathrm{pH} 2$ using dilute $\mathrm{HClO} 4$ and $\mathrm{NaOH}$, as needed. 


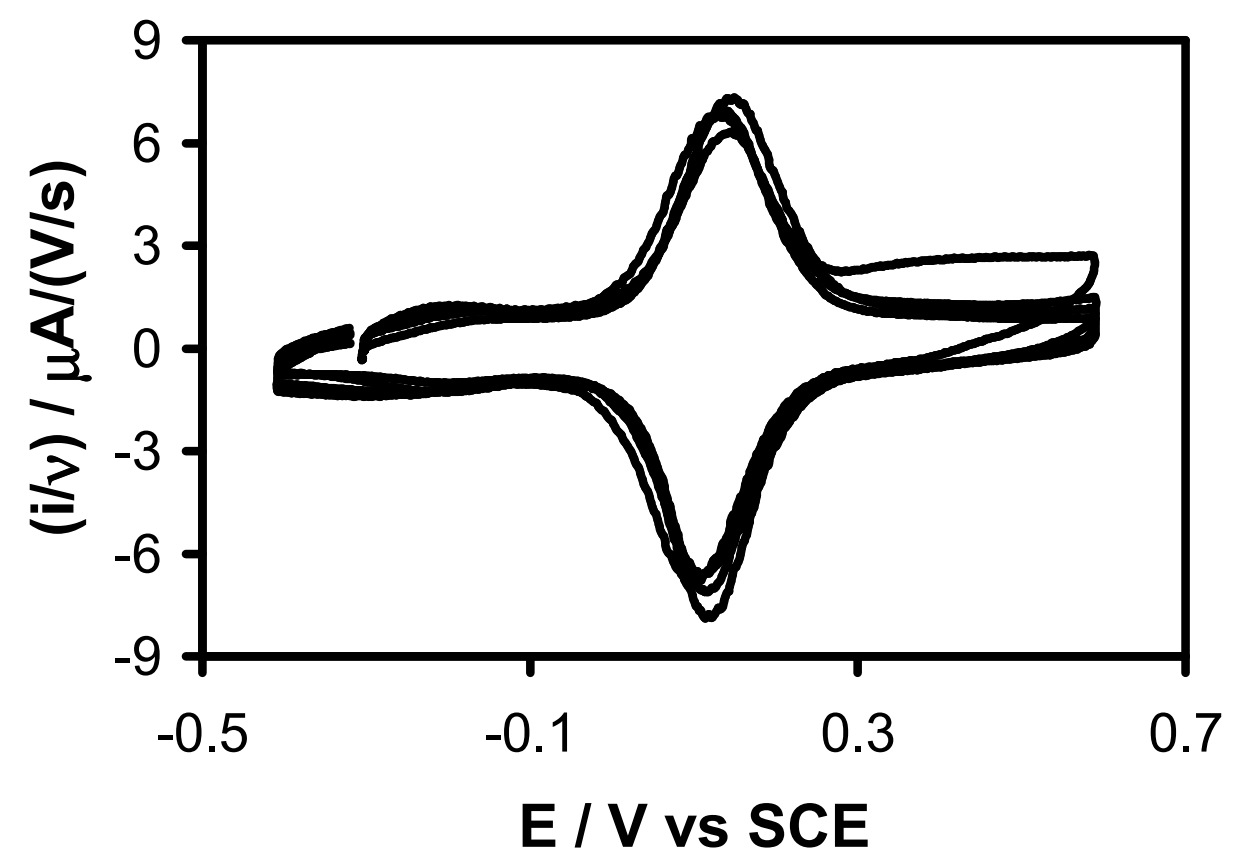

Figure 4.3.3. 3

Voltammograms of [Os(bpy)2(4-AMP)Cl] ${ }^{+}$at multiple $\mathrm{pH}$ 's at a scan rate of $.1 \mathrm{~V} / \mathrm{s}$. Electrolyte at all pH's was $1.0 \mathrm{M} \mathrm{NaClO}_{4}$. $\mathrm{pH}$ was adjusted using dilute $\mathrm{HClO} 4$ and $\mathrm{NaOH}$, as needed. CV's were collected at the following pH's: 2,4,6, and 9 . 


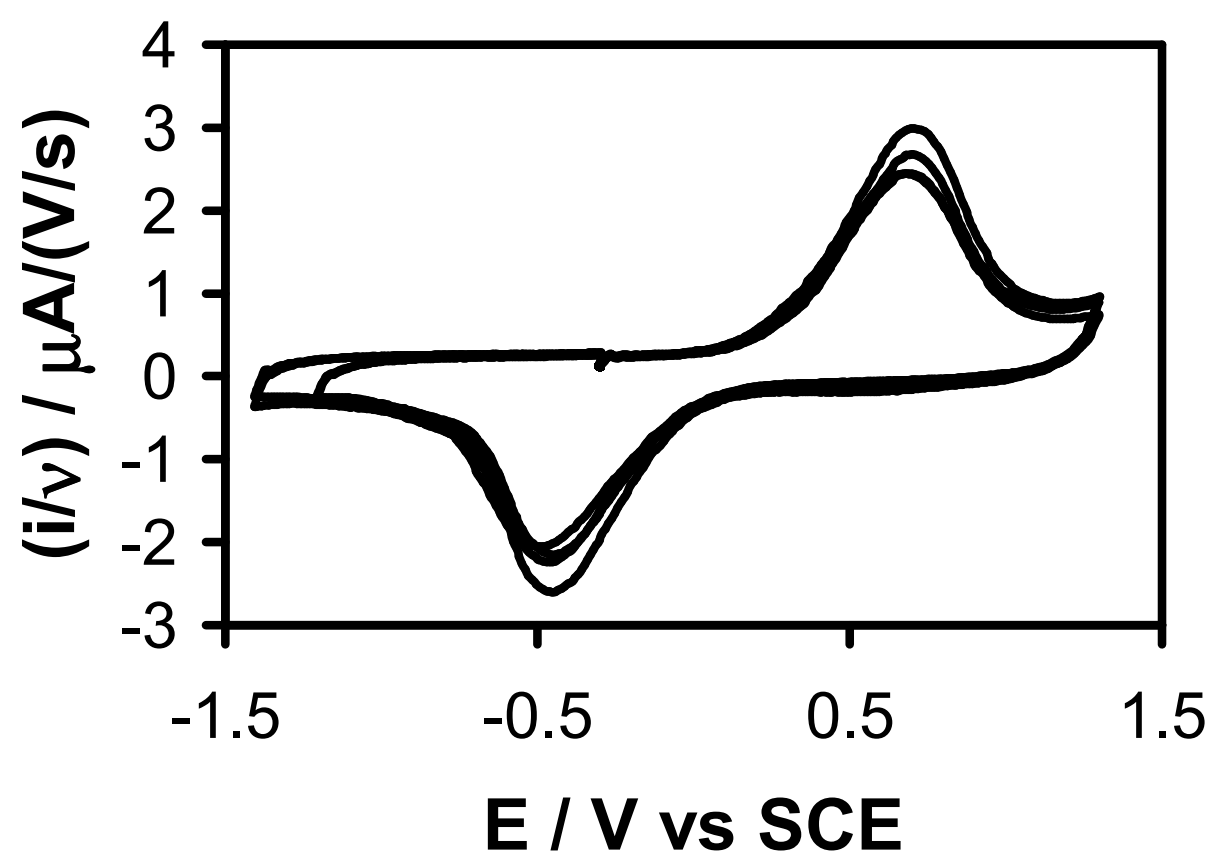

Figure 4.3.3. 4

Voltammograms of [Os(bpy)2(4-AMP)CI] $]^{+}$at multiple $\mathrm{pH}$ 's at a scan rate of $1000 \mathrm{~V} / \mathrm{s}$. . Electrolyte at all $\mathrm{pH}$ 's was $1.0 \mathrm{M} \mathrm{NaClO}_{4}$. $\mathrm{pH}$ was adjusted using dilute $\mathrm{HClO} 4$ and $\mathrm{NaOH}$, as needed. CV's were collected at the following $\mathrm{pH}$ 's: $2,4,6$, and 9 . 


\begin{tabular}{|c|c|c|c|}
\hline pH & $\begin{array}{c}\text { Standard Rate } \\
\text { Constant (k50 avg) }\left(\mathrm{s}^{-1}\right)\end{array}$ & $\begin{array}{c}\text { Reorganization } \\
\text { Energy - } \\
\text { cathodic (eV) }\end{array}$ & $\begin{array}{c}\text { Reorganization } \\
\text { Energy - } \\
\text { anodic(eV) }\end{array}$ \\
\hline 1.5 & 12.3 & 0.60 & 0.65 \\
\hline 1.9 & 8.9 & 0.55 & 0.70 \\
\hline 2.9 & 10.8 & 0.60 & 0.70 \\
\hline 4.3 & 9.5 & 0.60 & 0.65 \\
\hline 5.4 & 13.0 & 0.60 & 0.60 \\
\hline 6.5 & 11.3 & 0.55 & 0.60 \\
\hline 7.2 & 9.9 & 0.55 & 0.65 \\
\hline 9.1 & 9.9 & 0.55 & 0.65 \\
\hline 11.0 & 9.3 & 0.55 & 0.55 \\
\hline
\end{tabular}

\begin{tabular}{|c||c|}
\hline AVERAGE $\lambda$ & AVERAGE $\mathbf{k}_{\mathbf{s}}$ \\
\hline \hline $0.61 \pm 0.05 \mathrm{eV}$ & $10.5 \pm 1.4 \mathrm{~s}^{-1}$ \\
& \\
\hline
\end{tabular}




\subsubsection{Results - The $\left[\mathrm{Os}(\mathrm{tpy}-\mathrm{OH})(\mathrm{bpy}) \mathrm{OH}_{2}\right]^{2+}$ System}

The synthesis of $\left[\mathrm{Os}(\mathrm{tpy}-\mathrm{OH})(\mathrm{bpy}) \mathrm{OH}_{2}\right]\left(\mathrm{PF}_{6}\right)_{2}$ was ultimately unsuccessful. Initial voltammograms showed a $\mathrm{pH}$ dependency that qualitatively appeared to be correct. However, upon fitting the data, the slope of the data was super-nernstian $(<-60 \mathrm{mV} / \mathrm{pH})$, as seen if Figure 4.3.4.1. Also, the fitted value of $\mathrm{pK}_{\mathrm{a} 2}$ was larger than the expected value from the literature. This feature was compounded by the fact that we were never able to get a clean product, through synthesis or purification. Upon obtaining a mass spectrum of the product, it became obvious that the alkanethiol tether had been lost somewhere during the triflic acid reflux step. This might explain the abnormal $\mathrm{pH}$ dependency, since the coupling point on the tpy without the tether could introduce a second position where PCET could occur. The appearance of additional waves might be due to this new ET site. Because of the overwhelming problems, this approach was scrapped.

\subsubsection{Results - The $\left[\mathrm{Os}(\mathrm{bpy})_{2}(4-\mathrm{AMP}) \mathrm{OH}_{2}\right]^{2+}$ System}

Coupling the redox center to the mixed monolayer was fairly routine once the amount of EDC was minimized in the coupling reaction as discussed in Section 4.2.4. Figure 4.3.5.1 is indicative of a typical voltammogram of the $\mathrm{Os}^{2+/ 3+}$ wave for the attached redox couple (data analysis for the $\mathrm{Os}^{3+/ 4+}$ wave was omitted because of the overlap with gold oxidation). Typical reversible peak splittings, $\Delta \mathrm{E}_{\mathrm{p}}$, were less than 


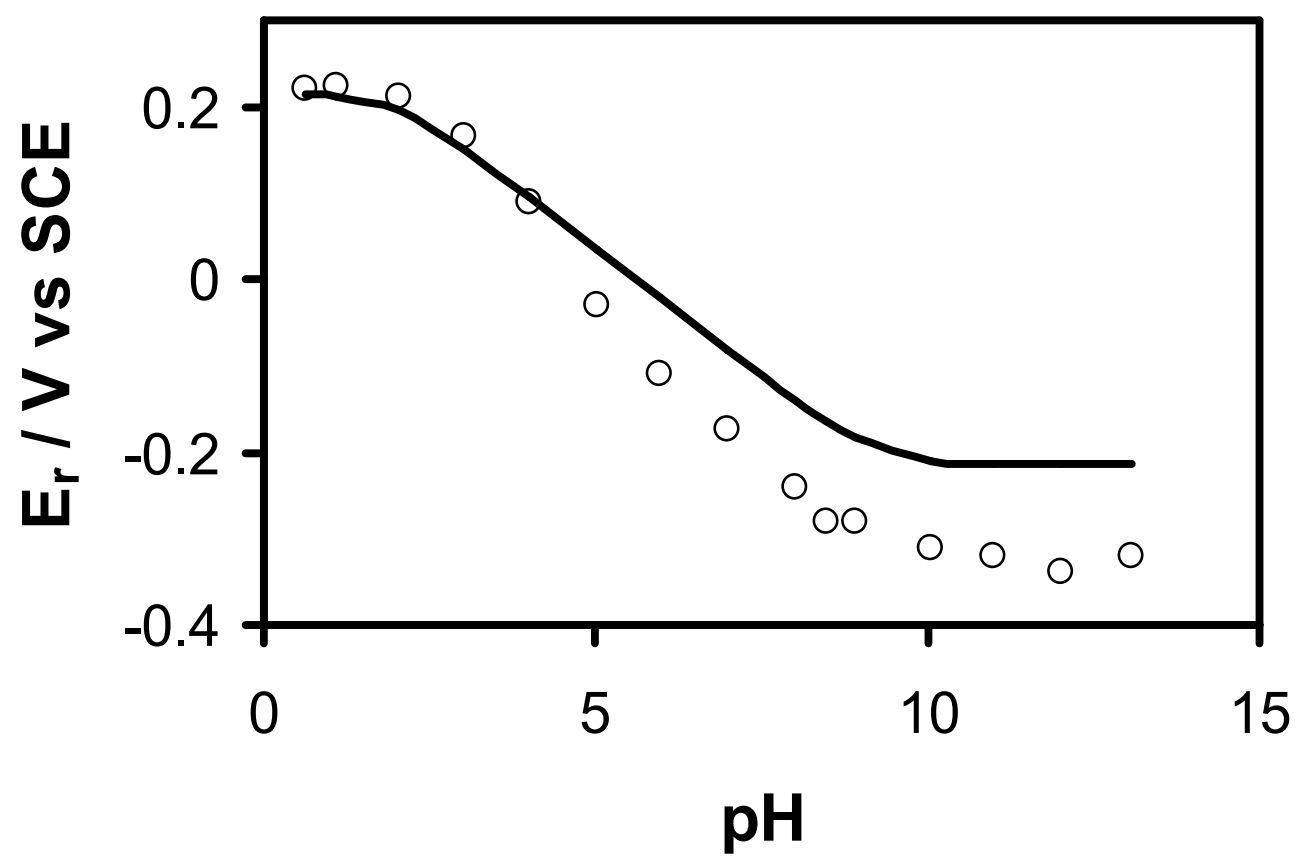

Figure 4.3.4. $1 \quad \mathrm{E} 1 / 2 \mathrm{vs} \mathrm{pH}$ for $\left[\mathrm{Os}(\mathrm{tpy}-\mathrm{OH})(\mathrm{bpy}) \mathrm{OH}_{2}\right]^{2+}$. The line is a theoretical fit to $\mathrm{E}_{1}{ }^{0}$ and $\mathrm{pK}_{\mathrm{a} 1}$, and $\mathrm{pK}_{\mathrm{a} 2}$. Clearly the formal potential decreases more rapidly with $\mathrm{pH}$ than the Nernstian $-59 \mathrm{mV} / \mathrm{pH}$. 


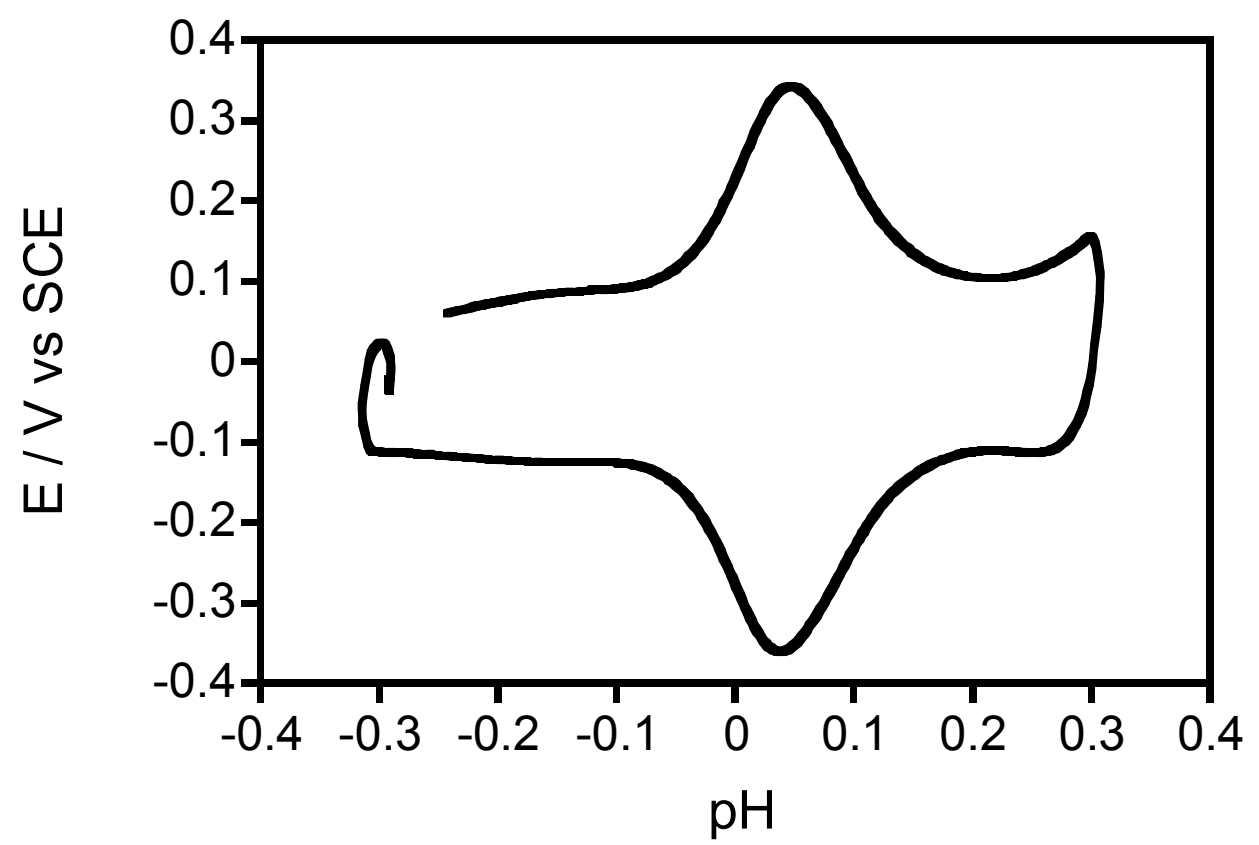

Figure 4.3.5. $1 \quad$ Typical Voltammogram of [Os(bpy)2(4-AMP)OH ${ }_{2}{ }^{2+}$ attached to a $50 / 50 \mathrm{HS}\left(\mathrm{CH}_{2}\right)_{12} \mathrm{OH} / \mathrm{HS}\left(\mathrm{CH}_{2}\right)_{15} \mathrm{COOH}$ Monolayer, $0.5 \mathrm{M} \mathrm{K}_{2} \mathrm{SO}_{4}+0.1 \mathrm{M}$ Britton-Robinson buffer at pH 6.85 . 
$17 \mathrm{mV}$. Typical FWHM values were about $100 \mathrm{mV}$. This ideal reversible peak shape occurred throughout the entire $\mathrm{pH}$ range. There was no peak broadening at high $\mathrm{pH}$ as seen in the galvinol system. The $\mathrm{pH}$ dependence of the formal potential is seen in Figure 4.3.5.2. From $\mathrm{pH} 3$ to $\mathrm{pH} 9, \mathrm{E}_{\mathrm{r}}$ shifts linearly with $\mathrm{pH}$ with a Nernstian slope of $60 \mathrm{mV} / \mathrm{pH} . \mathrm{E}_{\mathrm{r}}$ flattens out, towards $\mathrm{pH}$ independence, at $\mathrm{pH}<3$ and $\mathrm{pH}>9$. The solid line in the figure is a least squares fit of the data to the theoretical model. The fit yields an $\mathrm{E}_{1}{ }^{0^{\prime}}=+0.30 \mathrm{~V}$ vs SCE with a $\mathrm{pK}_{\mathrm{a} 1}=2.4$ and $\mathrm{E}_{2}{ }^{0^{\prime}}=-0.11 \mathrm{~V}$ vs SCE with a $\mathrm{pK}_{\mathrm{a} 2}=9.3$ Thus, the value for $\mathrm{pK}_{\text {mid }}=5.9$.

At all pH's, voltammograms were collected at scan rates from $0.1 \mathrm{~V} / \mathrm{s}$ to 1000 V/s, using 1, 2, 5 intervals. Figures 4.3.5.3, 4.3.5.4, 4.3.5.5, 4.3.5.6, and 4.3.5.7 illustrate the effect of scan rate $(0.1,1,10,100$, and $1000 \mathrm{~V} / \mathrm{s})$ on the shape of the voltammograms, at $\mathrm{pH} 0.82,4.01,5.89,8.08$, and 10.05 , respectively. These $\mathrm{pH}$ 's were chosen because they represent key pHs. Voltammograms at $\mathrm{pH} 0.82$ and 10.05 (which are outside the range where the complex is $\mathrm{pH}$ dependent) and 5.89 ( $\left.\mathrm{pK}_{\text {mid }}\right)$ should be symmetrical, with respect to the anodic and cathodic peaks.

Voltammograms at $\mathrm{pH} 4.01$ and 8.08 should exhibit distinct asymmetry in the voltammograms under kinetic control, with $\mathrm{pH} 4.01$ having a sharper, less shifted anodic peak, and $\mathrm{pH} 8.08$ having a sharper, less shifted cathodic peak. The first observation that can be made is that at no $\mathrm{pH}$ is the extreme asymmetry in peak shape seen that was apparent in the galvinol system. In fact, one would be hard pressed to make any judgements at all by visual inspection alone. It does appear that at the pH's below $\mathrm{pK}_{\text {mid }}$ that the cathodic peak is slightly broader than the anodic peak, and at 


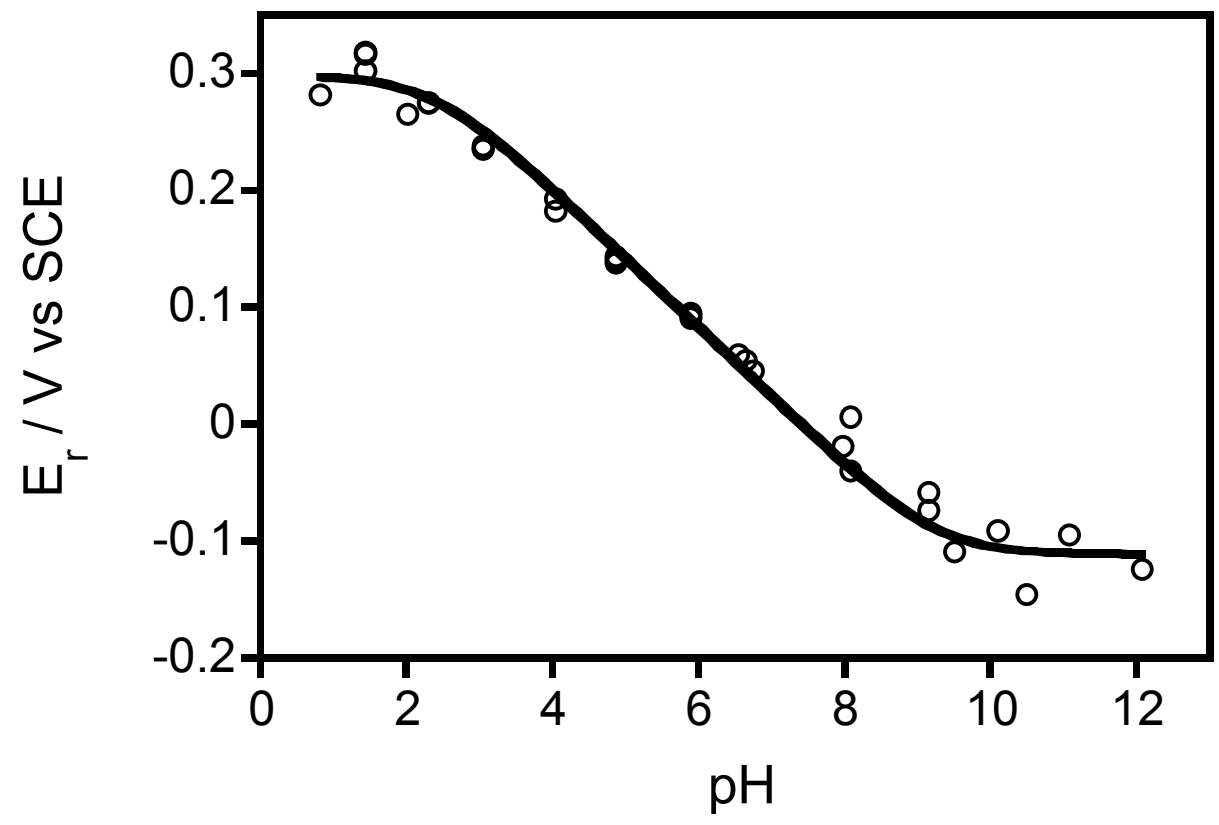

Figure 4.3.5. $2 \quad \mathrm{E}_{\mathrm{r}} \mathrm{vs} \mathrm{pH}$ for [Os(bpy $\left.)_{2}(4-\mathrm{AMP}) \mathrm{OH}\right]_{2}{ }^{2+}$ attached to a 50/50 $\mathrm{HS}\left(\mathrm{CH}_{2}\right)_{12} \mathrm{OH} / \mathrm{HS}\left(\mathrm{CH}_{2}\right)_{15} \mathrm{COOH}$ Monolayer 


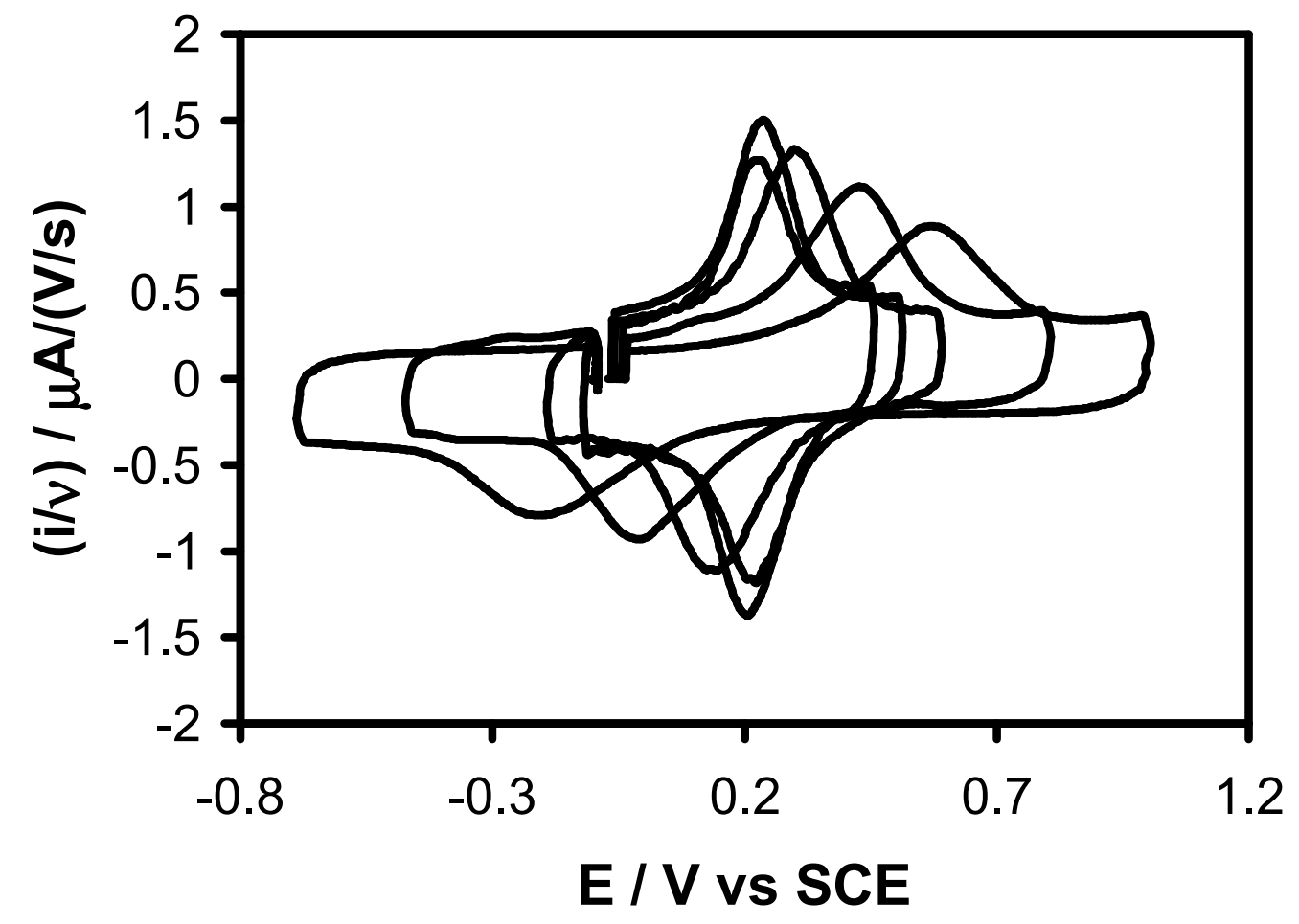

Figure 4.3.5. 3

Voltammograms at multiple scan rates in $0.5 \mathrm{M} \mathrm{K}_{2} \mathrm{SO}_{4}+$ $0.1 \mathrm{M}$ Britton-Robinson buffer $-\mathrm{pH}$ 0.82. Scan rates are $0.1,1,10,100$, and 1000 $\mathrm{V} / \mathrm{s}$. Currents are normalized with respect to the scan rate. 


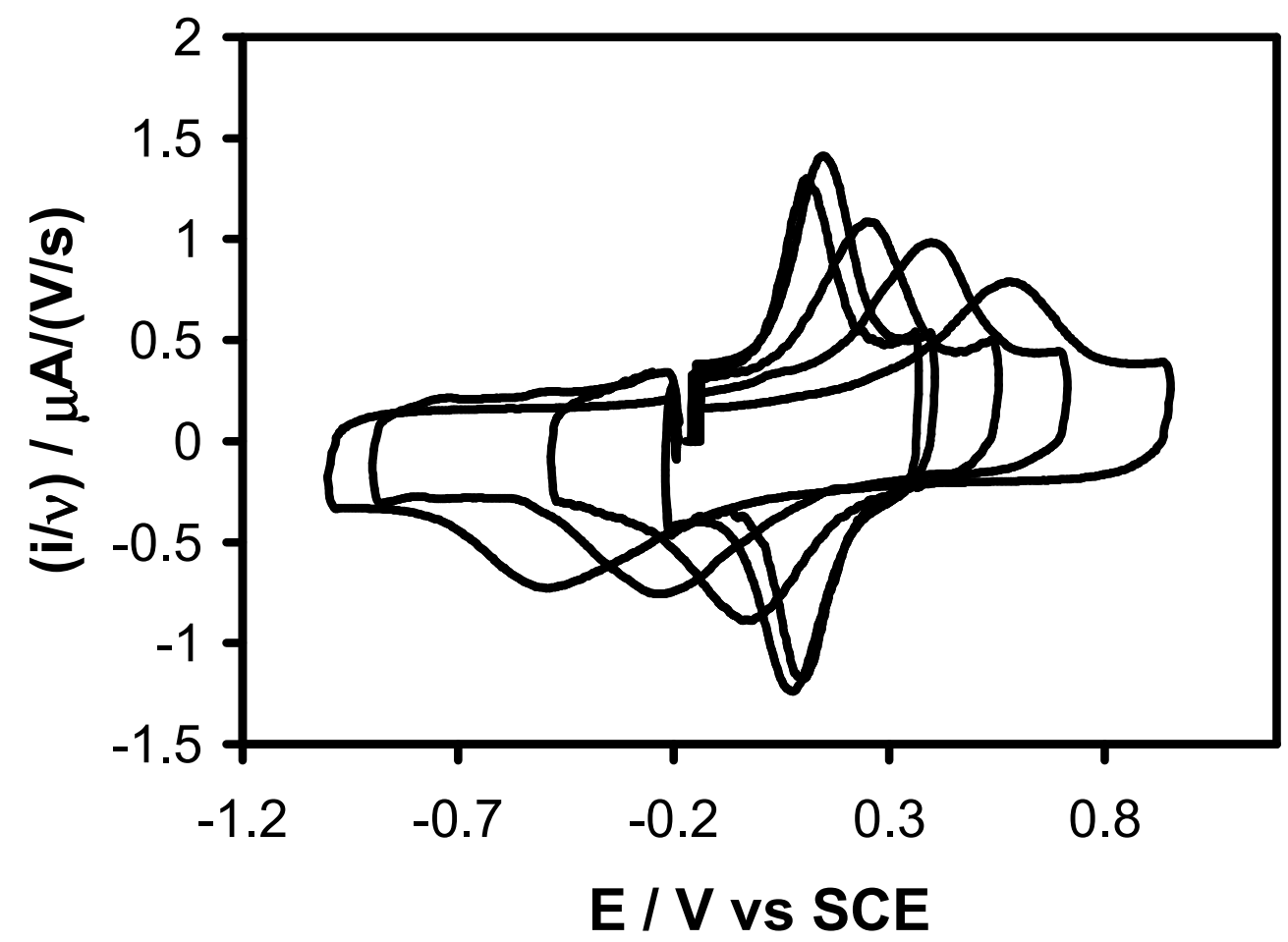

Figure 4.3.5. 4

Voltammograms at multiple scan rates in $0.5 \mathrm{M} \mathrm{K}_{2} \mathrm{SO}_{4}+$ $0.1 \mathrm{M}$ Britton-Robinson buffer $-\mathrm{pH}$ 4.01. Scan rates are $0.1,1,10,100$, and 1000 $\mathrm{V} / \mathrm{s}$. Currents are normalized with respect to the scan rate. 


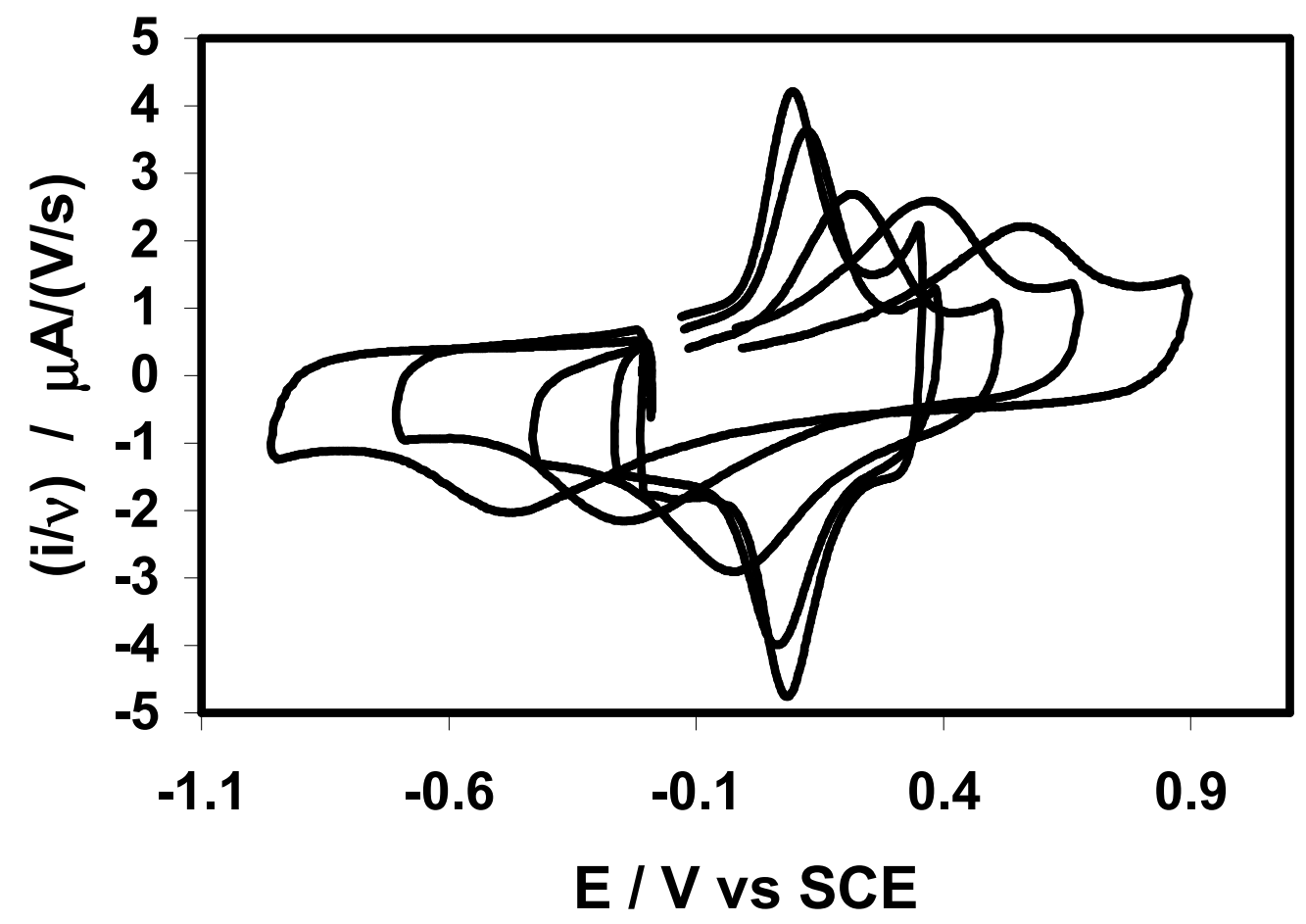

Figure 4.3.5. 5

Voltammograms at multiple scan rates in $0.5 \mathrm{M} \mathrm{K}_{2} \mathrm{SO}_{4}+$ $0.1 \mathrm{M}$ Britton-Robinson buffer $-\mathrm{pH}$ 5.89. Scan rates are $0.1,1,10,100$, and 1000 V/s. Currents are normalized with respect to the scan rate. 


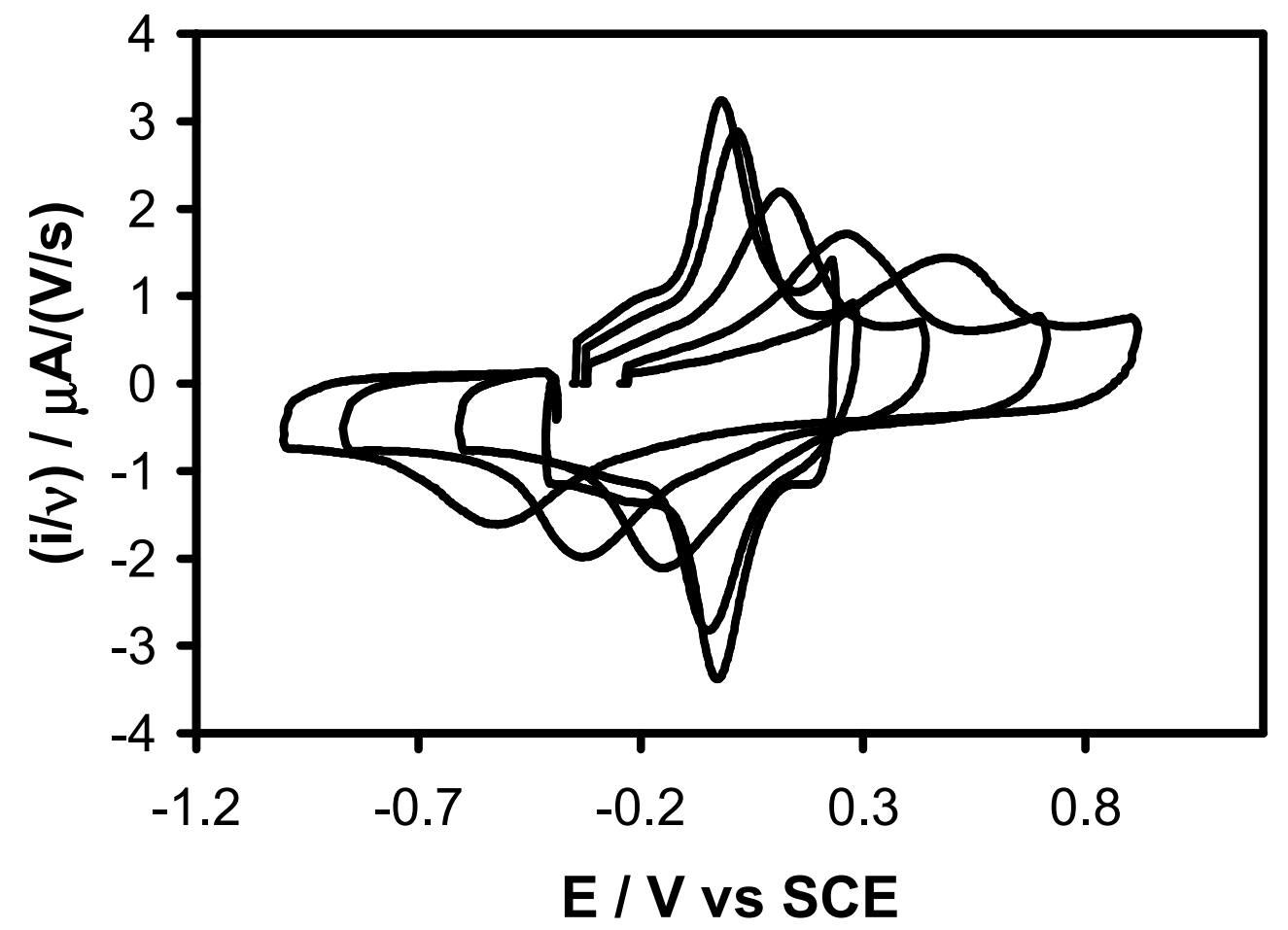

Figure 4.3.5. 6

Voltammograms at multiple scan rates in $0.5 \mathrm{M} \mathrm{K}_{2} \mathrm{SO}_{4}+$ $0.1 \mathrm{M}$ Britton-Robinson buffer $-\mathrm{pH}$ 8.08. Scan rates are $0.1,1,10,100$, and 1000 V/s. Currents are normalized with respect to the scan rate. 


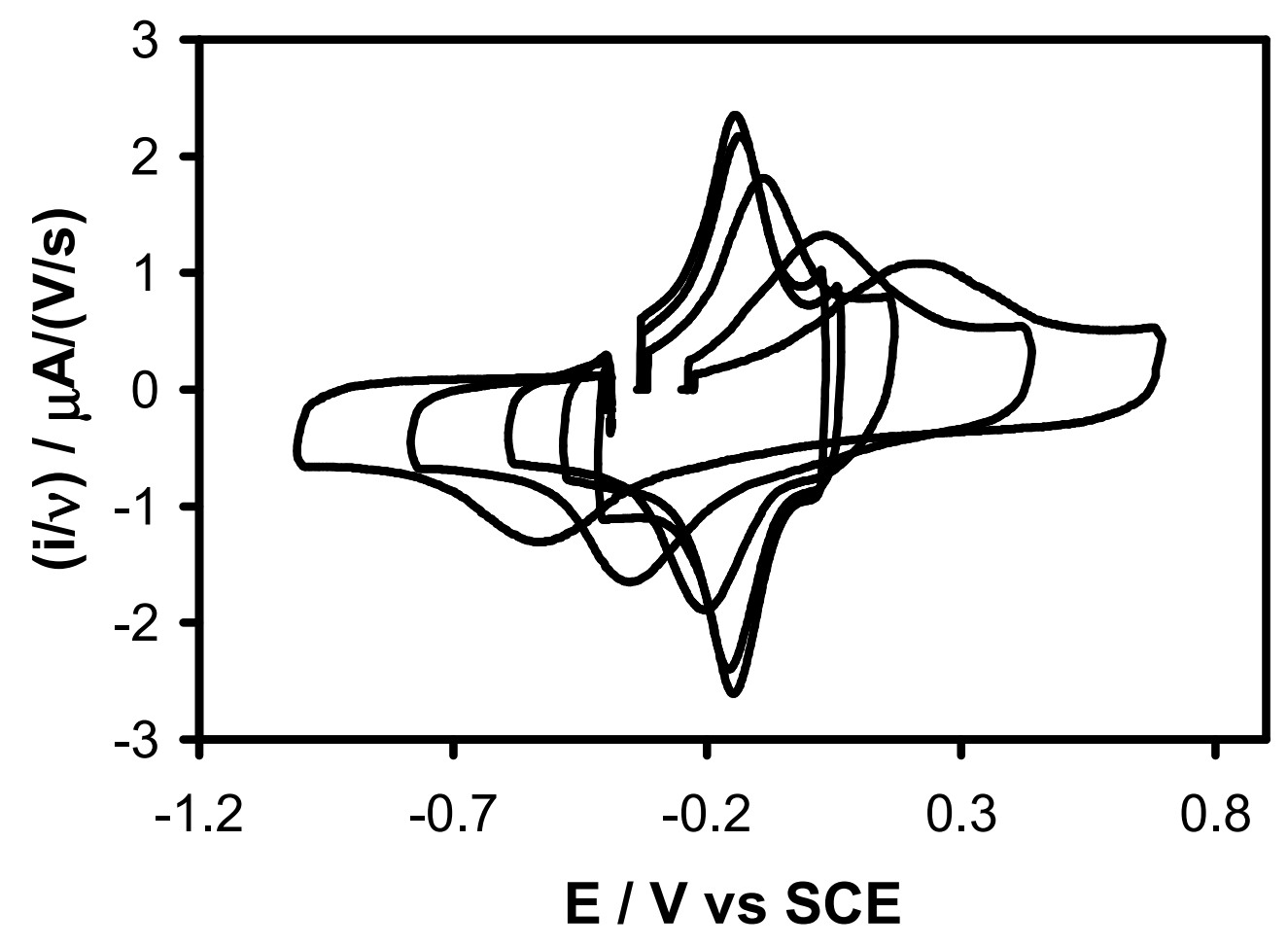

Figure 4.3.5. 7 Voltammograms at multiple scan rates in $0.5 \mathrm{M} \mathrm{K}_{2} \mathrm{SO}_{4}+$ $0.1 \mathrm{M}$ Britton-Robinson buffer $-\mathrm{pH}$ 10.50. Scan rates are $0.1,1,10,100$, and 1000 $\mathrm{V} / \mathrm{s}$. Currents are normalized with respect to the scan rate. 
pH's above $\mathrm{pK}_{\text {mid, }}$ that the anodic peak is slightly broader than the cathodic peak. However, this observation is barely noticeable and thus subject to question. For a more exact analysis, the peak potential for each branch, $E_{p, a}$ or $E_{p, c}$, is examined in reference to $E_{r}$ as the scan rate increases. Theoretically, in a symmetrical system, $E_{p, a}$ - $E_{r}$ will equal $E_{p, c}-E_{r}$ as the scan rate increases because each branch is shifting at the same rate. In the region of intermediate $\mathrm{pH}$ between $\mathrm{pK}_{\text {mid }}$ and $\mathrm{pK}_{\mathrm{a} 2},\left(\mathrm{E}_{\mathrm{p}, \mathrm{a}}-\mathrm{E}_{\mathrm{r}}\right)>$ $\left(E_{p, c}-E_{r}\right)$ because the anodic branch is shifting at a faster rate than the cathodic branch. Conversely, in the region of intermediate $\mathrm{pH}$ between $\mathrm{pK}_{\text {mid }}$ and $\mathrm{pK}_{\mathrm{a} 1},\left(\mathrm{E}_{\mathrm{p}, \mathrm{a}}\right.$ $\left.E_{r}\right)<\left(E_{p, c}-E_{r}\right)$. Figures 4.3.5.8, 4.3.5.9, 4.3.5.10, 4.3.5.11, and 4.3.5.12 are plots of $E_{p}-E_{r}$ vs pH at pH 0.82, 4.01, 5.89, 8.08, and 10.05, respectively. It can be seen quickly that the peak splitting behavior is not obeying the model. Instead, each branch appears to be relatively symmetrical until a breaking point is reached, usually between 10 and $100 \mathrm{~V} / \mathrm{s}$. At this point, the cathodic branch starts shifting out at a faster rate than the anodic branch. This phenomenon is seen at all pH's, which is contradictory to the model.

The apparent standard rate constant was measured as a function of $\mathrm{pH}$ using both extraction methods. The results obtained by measuring the apparent standard rate constant at zero overpotential is seen in Figure 4.3.5.13. Fitting the data to the model (the solid line) results in a $\mathrm{k}_{\mathrm{s} 1}$ and $\mathrm{k}_{\mathrm{s} 2}=60 \mathrm{~s}^{-1}$. Only the data from $\mathrm{pH}<3$ and $\mathrm{pH}$ $>8$ was used in the fit. In the fit it was assumed that $k_{\mathrm{s} 1}=k_{\mathrm{s} 2}$. Again, the values of $\mathrm{k}_{\mathrm{s} 2}$ and $k_{s 1}$ are independent of whether the transfer coefficient is constant or if it is potential 


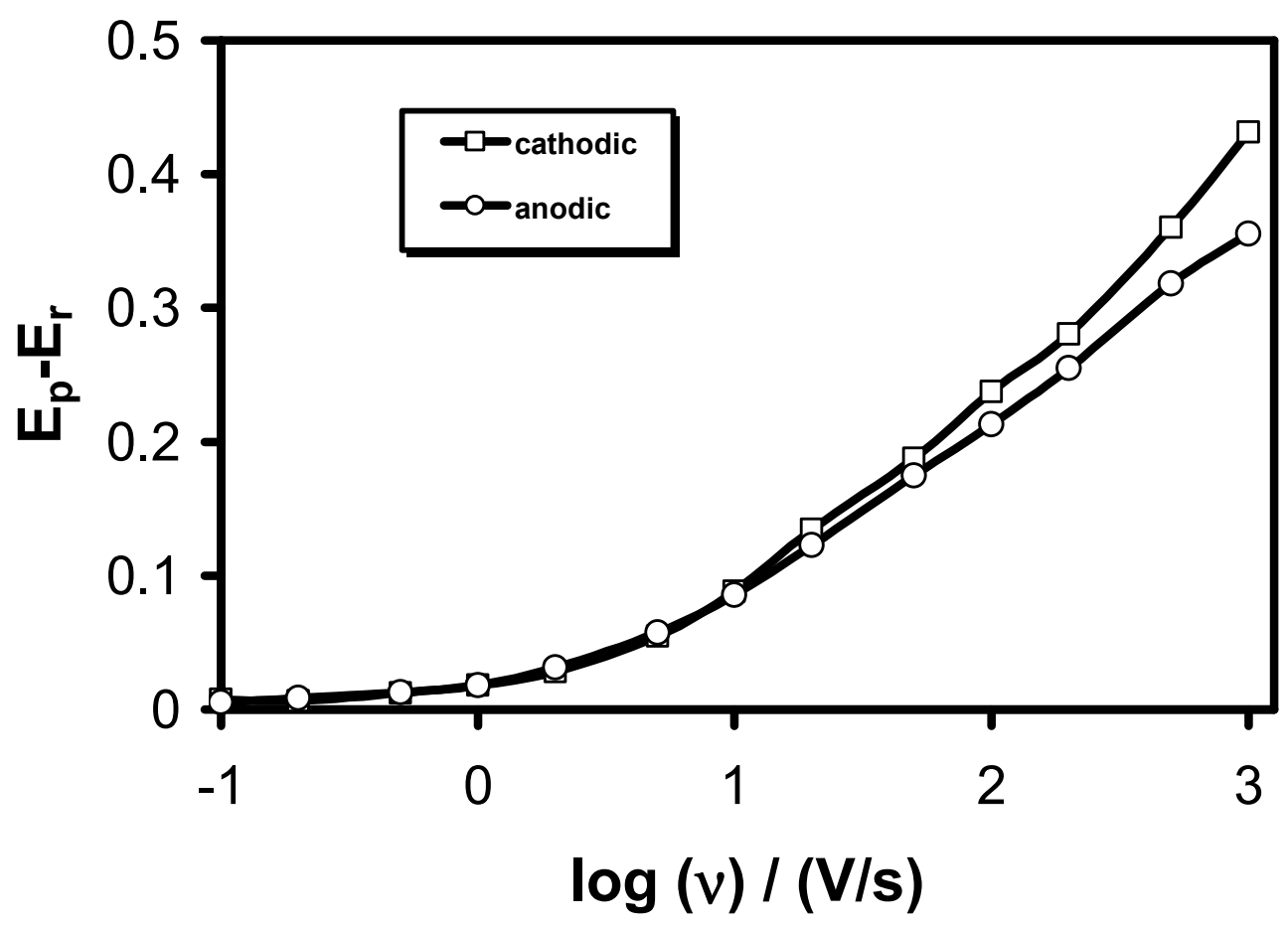

Figure 4.3.5. 8

$\left(E_{p}-E_{r}\right)$ vs Scan Rate - pH 0.82 


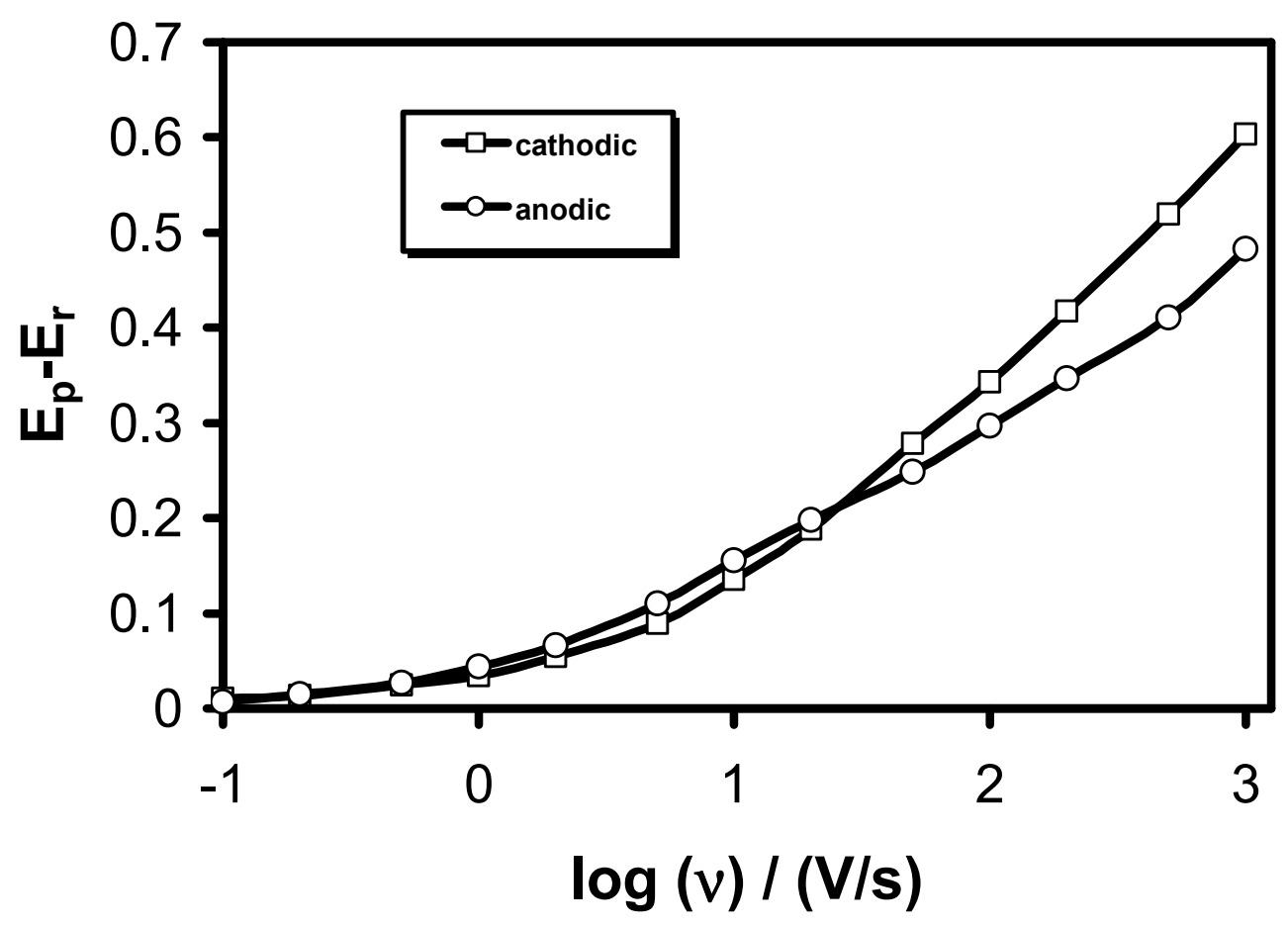

Figure 4.3.5. 9

$\left(E_{p}-E_{r}\right)$ vs Scan Rate - pH 4.01 


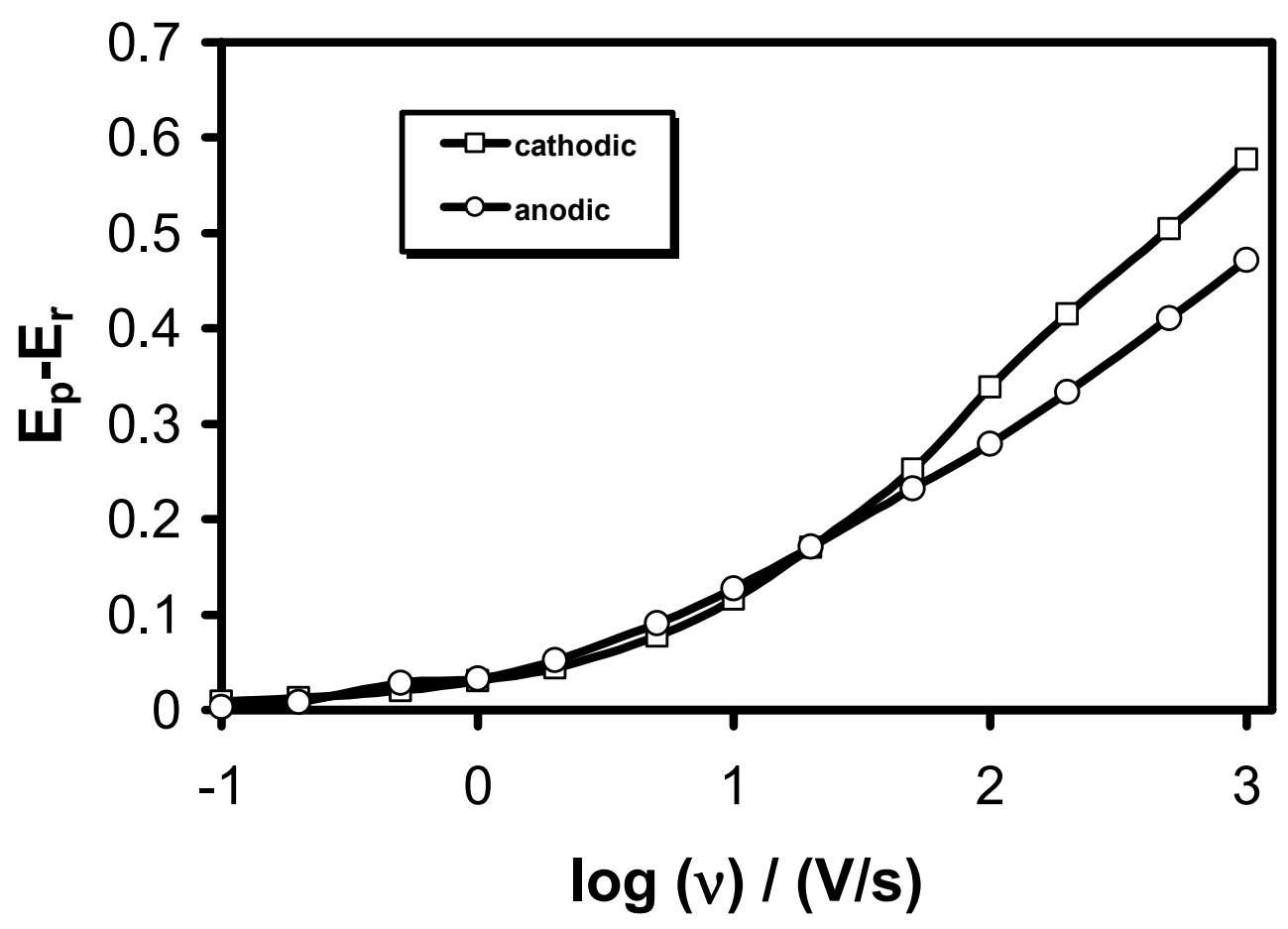

Figure 4.3.5. 10

$\left(E_{p}-E_{r}\right)$ vs Scan Rate - pH 5.89 


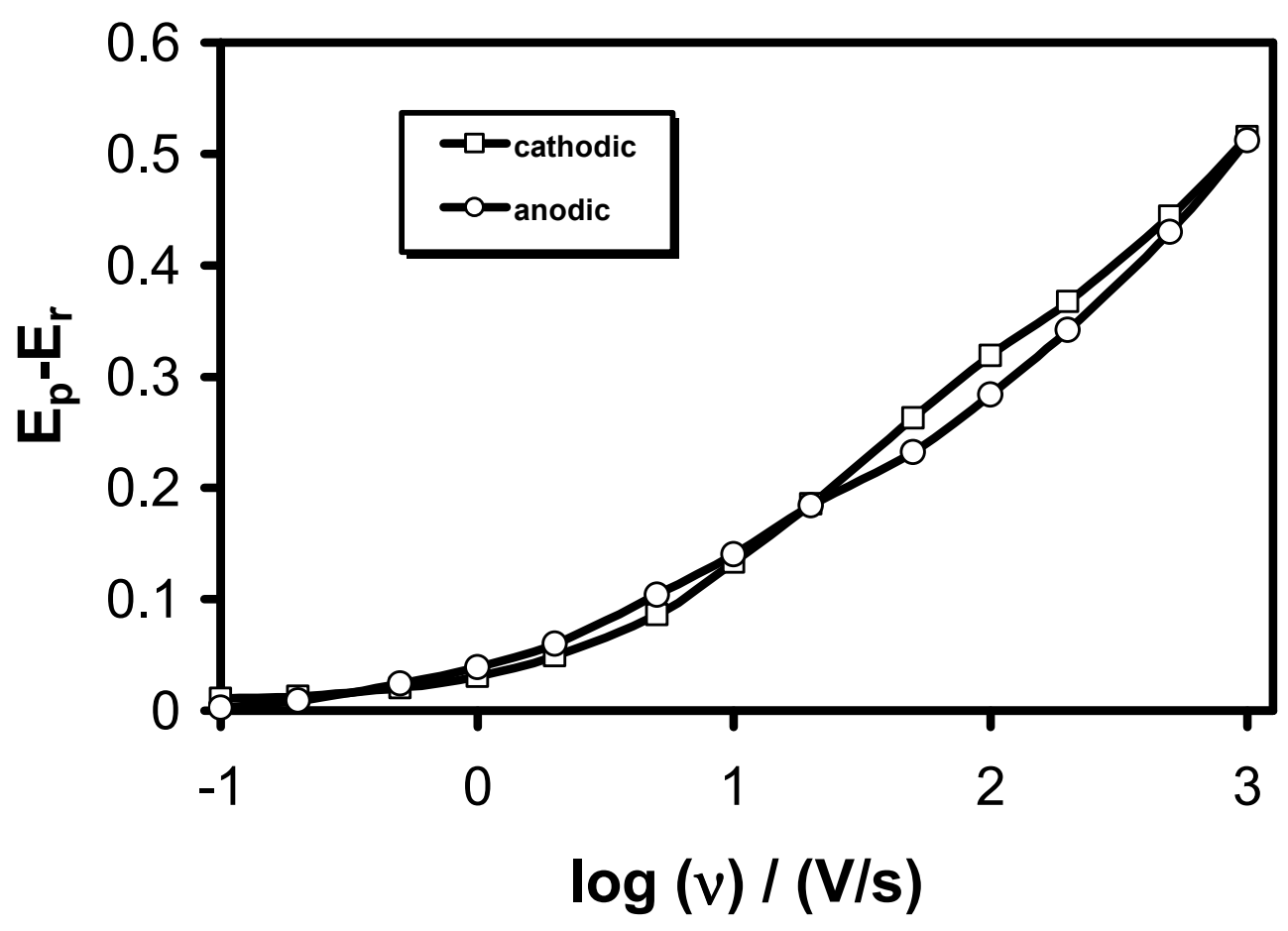

Figure 4.3.5. 11

$\left(E_{p}-E_{r}\right)$ vs Scan Rate - pH 8.02 


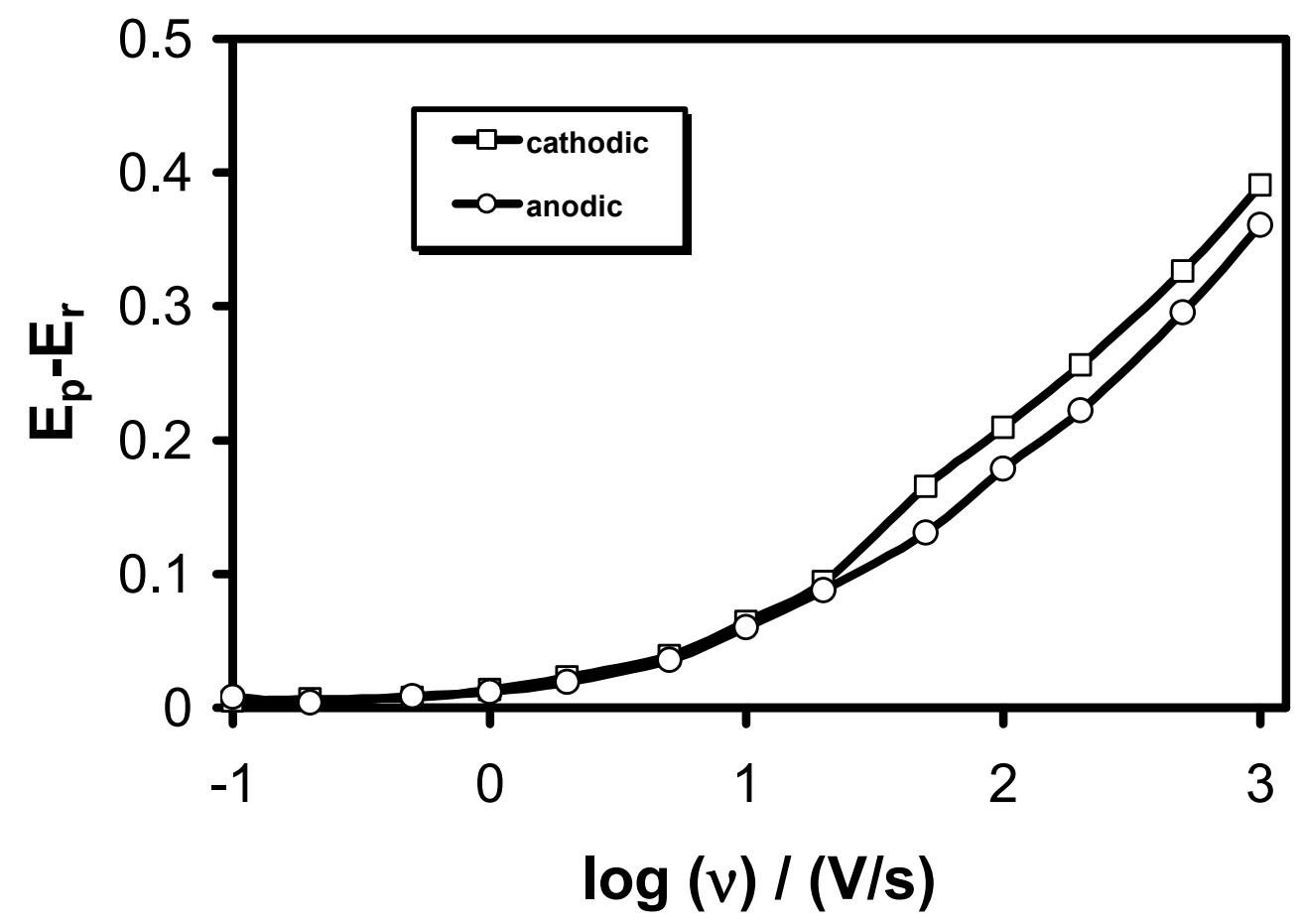

Figure 4.3.5. 12

$\left(E_{p}-E_{r}\right)$ vs Scan Rate - pH 10.50 


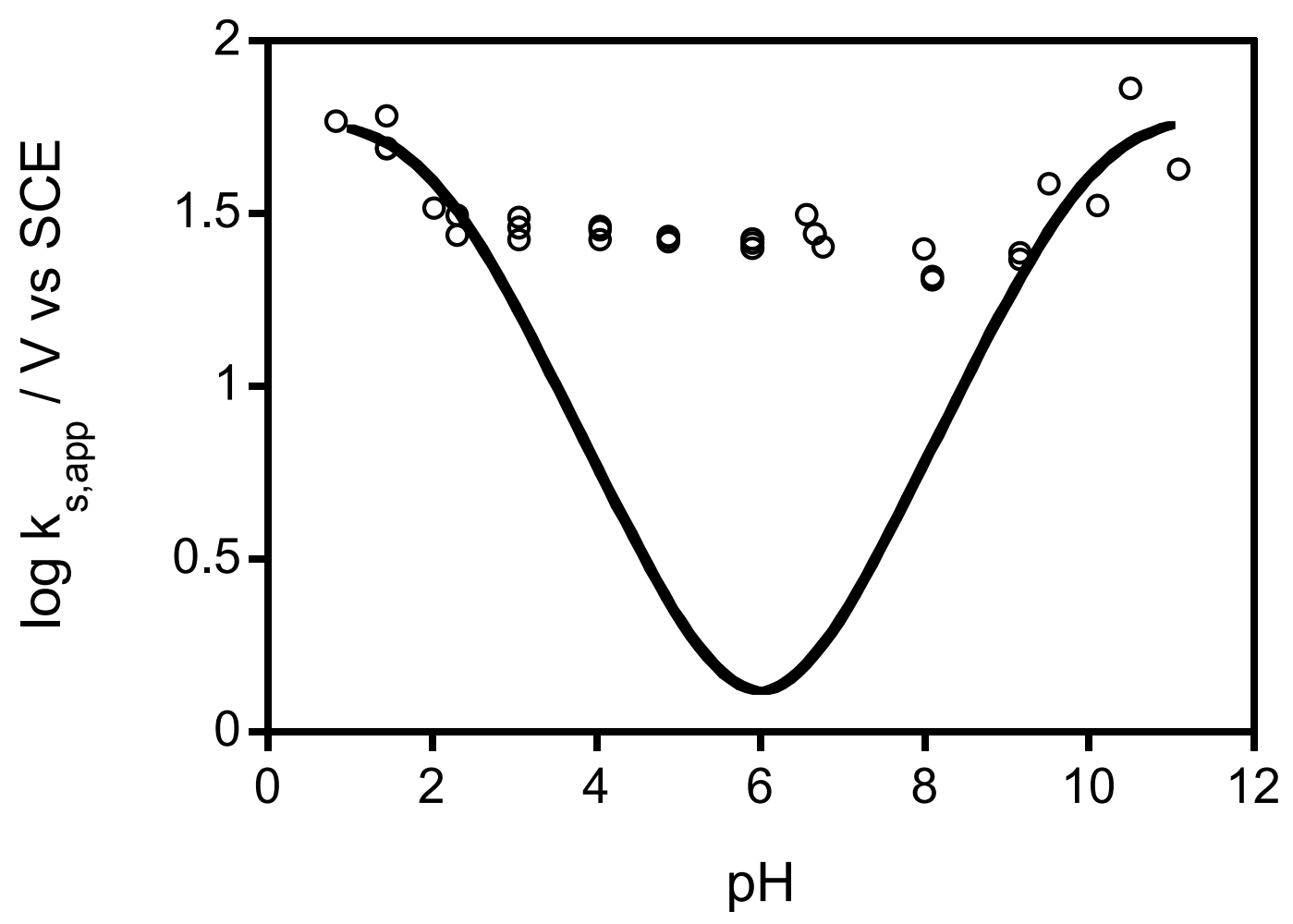

Figure 4.3.5. $13 \quad \log \mathrm{k}_{\mathrm{s}, \mathrm{app}} \mathrm{vs} \mathrm{pH}$ for $\left[\mathrm{Os}(\mathrm{bpy})_{2}(4-\mathrm{AMP}) \mathrm{OH}\right]_{2}{ }^{2+}$ attached to a $50 / 50 \mathrm{HS}\left(\mathrm{CH}_{2}\right){ }_{12} \mathrm{OH} / \mathrm{HS}\left(\mathrm{CH}_{2}\right)_{15} \mathrm{COOH}$ Monolayer $-\eta=0$ Analysis 
dependent. The solid line in Figure 4.3.5.13 is obtained with $\alpha=0.5$ at all potentials. Apparent standard rate constants from Tafel analysis are seen in Figure 4.3.5.14. Note that the Tafel results are qualitatively similar to the results obtained at the formal potential. Fitting the data to the model (the solid line) results in a $\mathrm{k}_{\mathrm{s} 1}$ and $\mathrm{k}_{\mathrm{s} 2}=30 \mathrm{~s}^{-1}$. By the nature of the Tafel analysis, slightly lower values are expected. Note that because $k_{s 2}$ is less than $100 \mathrm{~s}^{-1}$, the high $\mathrm{pH}$ deviation of $\mathrm{k}_{\mathrm{s}, \text { app }}$ seen in the galvinol project is absent here, and the two analysis techniques are much more comparable.

In the Osmium project, Tafel plots do show visible curvature, thus it is possible to gather information about the reorganization energy of the system. Figures 4.3.5.15, 4.3.5.16, and 4.3.5.17 are experimental Tafel plots taken at $\mathrm{pH}<\mathrm{pK}_{\mathrm{a} 1}, \mathrm{pH}=\mathrm{pK}_{\text {mid }}$, and $\mathrm{pH}>\mathrm{pK}_{\mathrm{a} 2}$. Table 4.3.5.1 is a table listing the calculated reorganization energy of the system as a function of $\mathrm{pH}$. Note that the $\lambda$ values are listed only at $\mathrm{pH}<\mathrm{pK}_{\mathrm{a} 1}$ and $\mathrm{pH}$ $>\mathrm{pK}_{\mathrm{a} 2}$. Because of the deviation of the apparent standard rate constant from the theory at $\mathrm{pK}_{\mathrm{a} 1}<\mathrm{pH}<\mathrm{pK}_{\mathrm{a} 2}$, values at intermediate $\mathrm{pH}$ are not listed.

Because of the presence of visible curvature, the apparent transfer coefficient at the formal potential cannot be estimated using the Butler-Volmer method as done in the galvinol project. However, it can be estimated using eqs (1.8.1) and (1.8.2). Using the overpotential and log $k(\eta)$ values from the Tafel analysis, one can solve for $\alpha(\eta)$ as long as one knows $\mathrm{k}_{\mathrm{s}, \mathrm{app}}$. For example (for the cathodic branch),

$$
\alpha(\eta)=\frac{\frac{-2.3 R T}{F}\left[\log k(\eta)-\log k_{s}\right]}{\eta}
$$




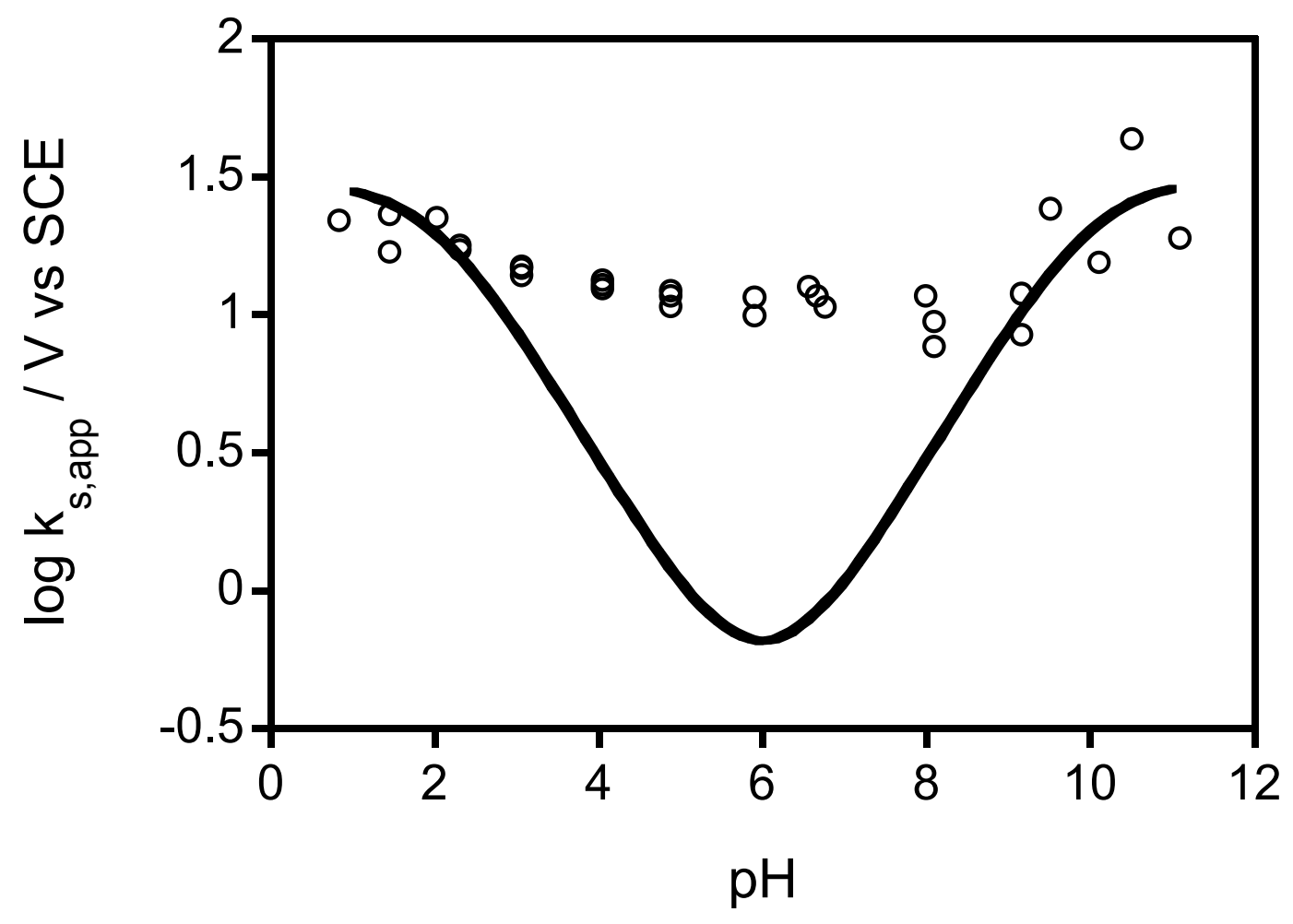

Figure 4.3.5. $14 \quad \log \mathrm{k}_{\mathrm{s}} \mathrm{vs} \mathrm{pH}$ for $\left[\mathrm{Os}(\mathrm{bpy})_{2}(4-\mathrm{AMP}) \mathrm{OH}\right]_{2}{ }^{2+}$ attached to a $50 / 50 \mathrm{HS}\left(\mathrm{CH}_{2}\right)_{12} \mathrm{OH} / \mathrm{HS}\left(\mathrm{CH}_{2}\right)_{15} \mathrm{COOH}$ Monolayer - Tafel Analysis 


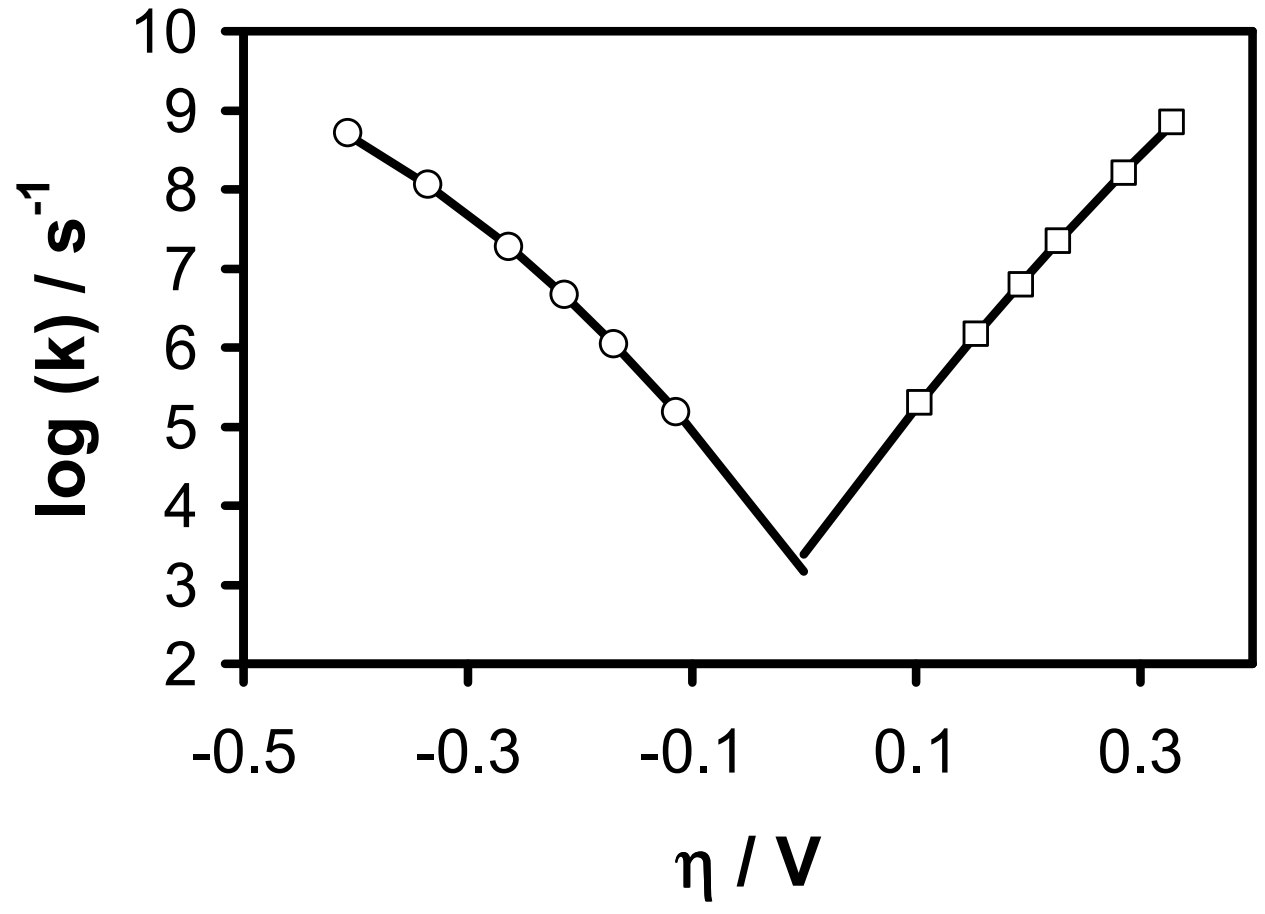

Figure 4.3.5. 15 Tafel plot at $\mathrm{pH} \mathbf{0 . 8 2}$. The solid lines are theoretical fits to the Marcus DOS model with $\lambda=1.0 \mathrm{eV}$ for the anodic branch and $\lambda=0.5 \mathrm{eV}$ for the cathodic branch. 


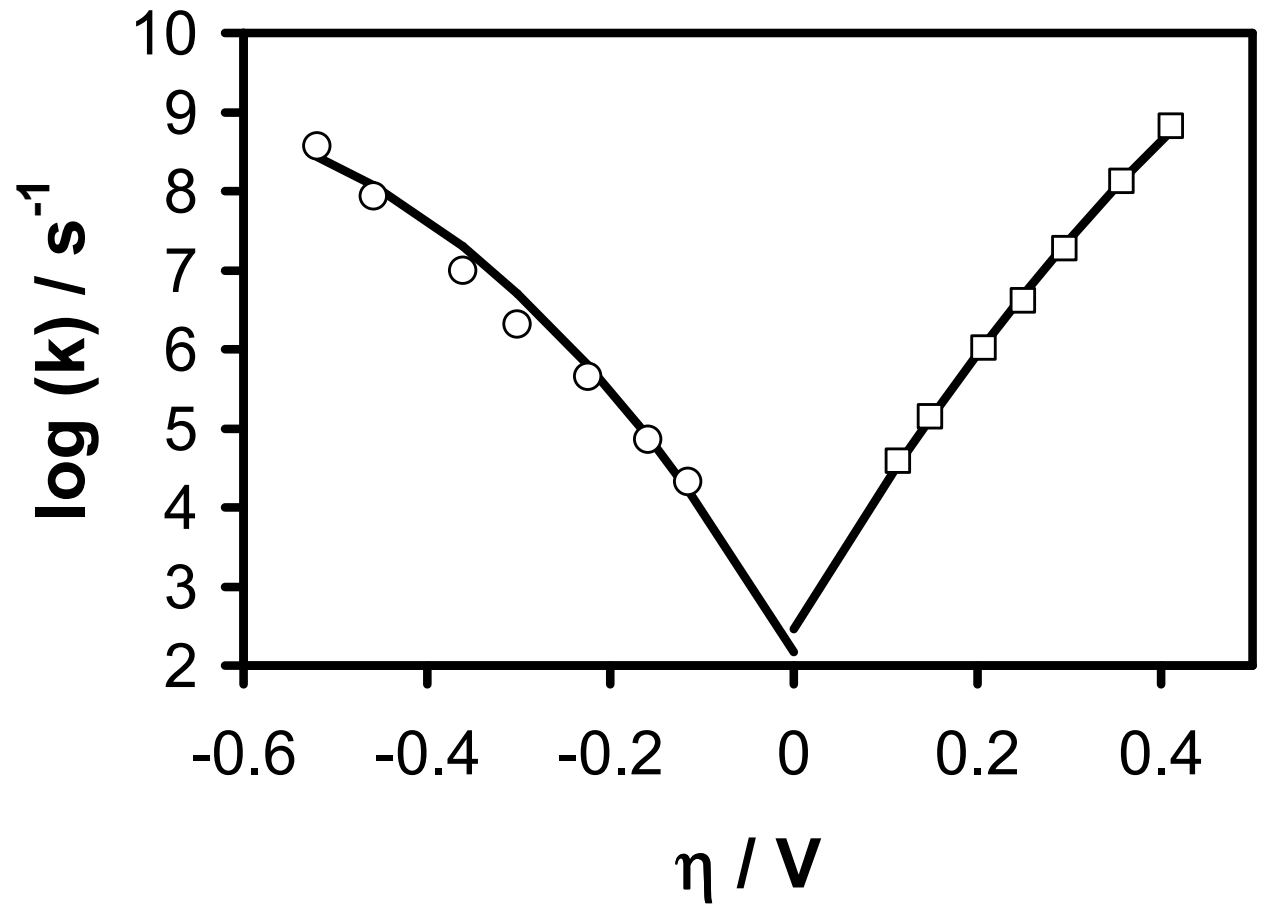

Figure 4.3.5. 16

Tafel Plot at $\mathrm{pH}=5.89$. The solid lines are theoretical fits to the Marcus DOS model with $\lambda=0.70 \mathrm{eV}$ for the anodic branch and $\lambda=0.50$ $\mathrm{eV}$ for the cathodic branch. 


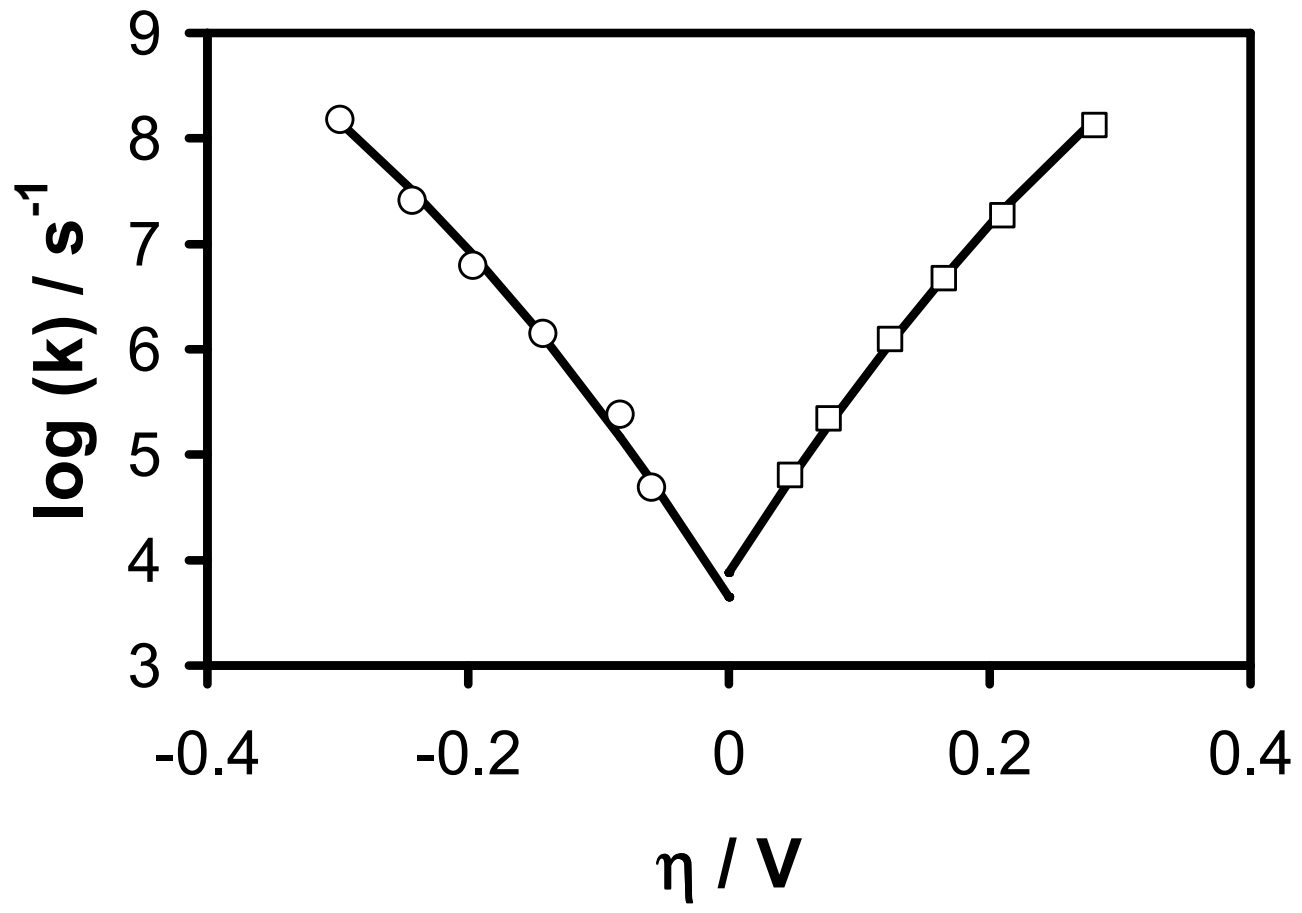

Figure 4.3.5. 17

Tafel Plot at $\mathrm{pH}$ 10.50. The solid lines are theoretical fits to the Marcus DOS model with $\lambda=0.70 \mathrm{eV}$ for the anodic branch and $\lambda=0.70$ eV for the cathodic branch. 
Table 4.3.5. 1

Reorganization Energy vs pH

\begin{tabular}{|c||c||c|}
\hline $\mathbf{p H}$ & $\begin{array}{c}\text { Cathodic Reorganization } \\
\text { Energy, eV }\end{array}$ & $\begin{array}{c}\text { Anodic Reorganization } \\
\text { Energy, eV }\end{array}$ \\
\hline 0.82 & 0.55 & 1.0 \\
2.02 & 0.55 & 0.75 \\
1.44 & 0.55 & 1.0 \\
1.44 & 0.55 & 0.90 \\
2.30 & 0.55 & 0.85 \\
2.30 & 0.55 & 0.85 \\
10.10 & 0.70 & 0.70 \\
10.50 & 0.55 & 0.55 \\
11.08 & 0.70 & 0.90 \\
\hline
\end{tabular}

\begin{tabular}{|c|c|c|c|}
\hline $\begin{array}{c}\text { Average low } \mathrm{pH} \lambda \\
\text { (Cathodic) }\end{array}$ & $\begin{array}{c}\text { Average low } \mathrm{pH} \lambda \\
\text { (Anodic) }\end{array}$ & $\begin{array}{c}\text { Average high } \mathrm{pH} \lambda \\
\text { (Cathodic) }\end{array}$ & $\begin{array}{c}\text { Average high } \mathrm{pH} \lambda \\
\text { (Anodic) }\end{array}$ \\
\hline $0.55 \pm 0 \mathrm{eV}$ & $0.89 \pm 0.10 \mathrm{eV}$ & $0.65 \pm 0.09 \mathrm{eV}$ & $0.72 \pm 0.17 \mathrm{eV}$ \\
\hline
\end{tabular}


Under the right conditions the experimental $\alpha(\eta)$ can be plotted versus overpotential to yield a nearly straight line of a slope $m$, and an intercept of $\alpha(0)$. A function is created that performed a best-fit linear regression by solving for two parameters, $\mathrm{k}_{\mathrm{s}, \mathrm{app}}$, and $m$. This procedure assumes that the anodic and cathodic branches of the Tafel plot have the same $k_{s, a p p .}$ The linearity of $\alpha(\eta) v s . \eta$ is valid to moderate overpotentials $(<0.5 \mathrm{~V})$. At higher overpotentials, the plot might exhibit significant curvature, depending upon the reorganization energy of the complex. The slope of the linear regression fit of the $\alpha(\eta)$ vs. $\eta$ plot is, for simple electron transfer reactions, a function of the reorganization energy. In other words, the slope $\mathrm{m}$ can be translated into a reorganization energy. A plot of $\alpha(0) v s \mathrm{pH}$ is seen in Figure 4.3.5.18. Table 4.3.5.2 lists the slope and the equivalent reorganization energy for the Tafel data at the extreme pH's. Again, it is clear that the reorganization energy deviates from 0.5 under the extreme $\mathrm{pH}$ conditions where the redox couple exhibits simple electron transfer. The cause for this deviation is unknown. It is worth noting that the $\mathrm{k}_{\mathrm{s}, \mathrm{app}}$ gathered from this analysis are comparable to values generated from the Tafel analysis.

\subsubsection{Discussion of Results}

Thermodynamically, this system follows the model. The apparent formal potential exhibits the expected $\mathrm{pH}$ independence at low $\mathrm{pH}$ 's $\left(\mathrm{pH}<\mathrm{pK}_{\mathrm{a} 1}\right), \mathrm{pH}$ dependence with a Nernstian slope at intermediate $\mathrm{pH}^{\prime} \mathrm{s}\left(\mathrm{pK}_{\mathrm{a} 1}<\mathrm{pH}<\mathrm{pK}_{\mathrm{a} 2}\right)$, and $\mathrm{pH}$ independence at high $\mathrm{pH}$ 's $\left(\mathrm{pK}_{\mathrm{a} 2}<\mathrm{pH}\right)$. It is worth noting that this system behaves 


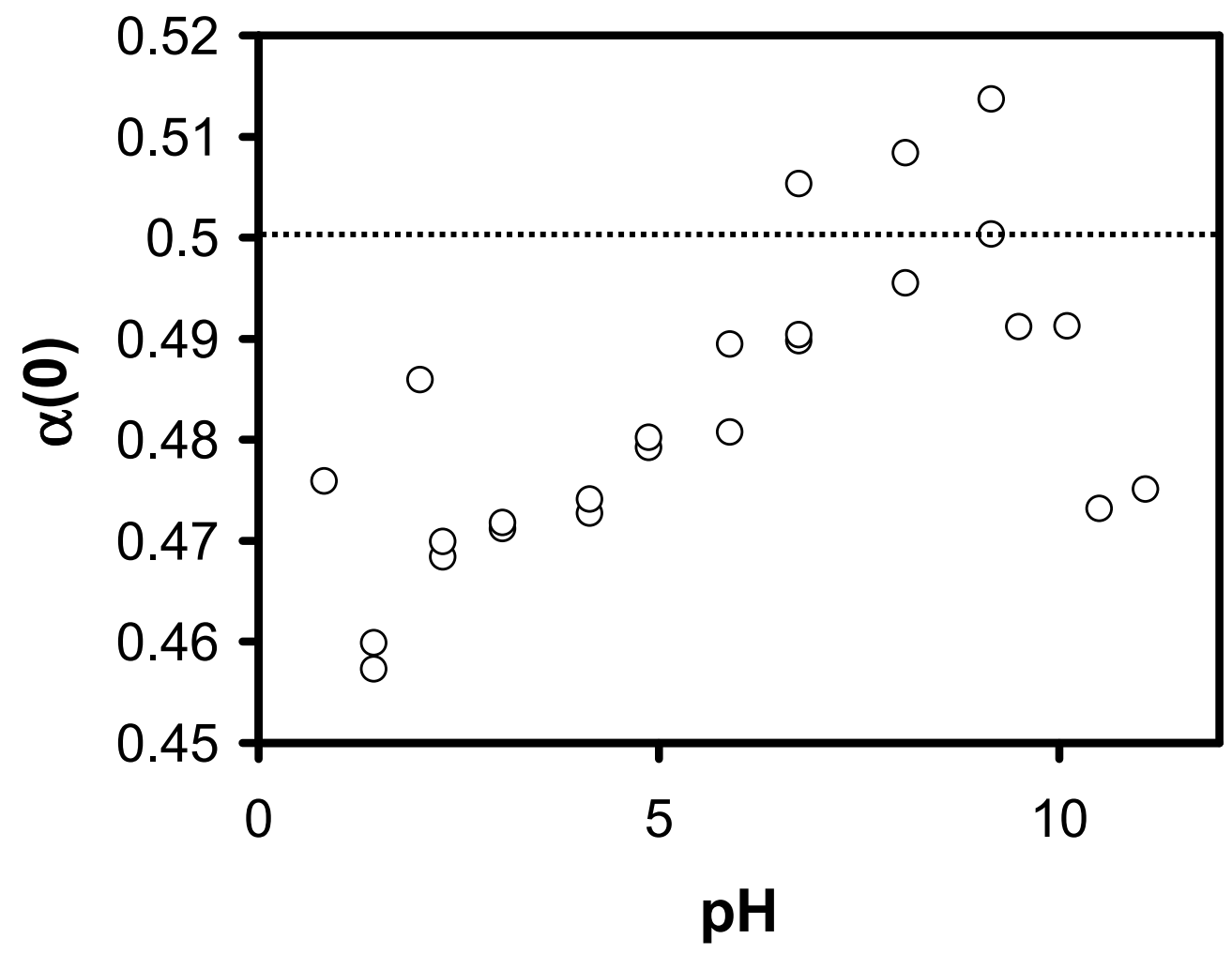

Figure 4.3.5. 18 $\alpha(0)$ vs $\mathrm{pH}$ 
Table 4.3.5. 2

Calculation of $\lambda$ using Alternate analysis for $\alpha(0)$

\begin{tabular}{|c||c|c|}
\hline $\mathbf{p H}$ & Slope, $\boldsymbol{m}$ & $\boldsymbol{\lambda}$ calculated from $\boldsymbol{m}(\mathbf{e V})$ \\
\hline 0.82 & 0.304 & 0.73 \\
\hline 1.44 & 0.332 & 0.66 \\
\hline 1.44 & 0.282 & 0.79 \\
\hline 2.02 & 0.332 & 0.66 \\
\hline 2.30 & 0.330 & 0.66 \\
\hline 2.30 & 0.335 & 0.65 \\
\hline 10.10 & 0.265 & 0.84 \\
\hline 10.50 & 0.349 & 0.62 \\
\hline 11.08 & 0.241 & 0.92 \\
\hline
\end{tabular}

\begin{tabular}{|c|c|}
\hline Average low $\mathrm{pH} \lambda$ & Average high $\mathrm{pH} \lambda$ \\
\hline $0.69 \pm 0.06 \mathrm{eV}$ & $0.79 \pm 0.16 \mathrm{eV}$ \\
\hline
\end{tabular}


slightly differently from the free solution parent compound, $[\mathrm{Os}(\mathrm{tpy})(\mathrm{bpy}) \mathrm{OH}]^{2+}$. This compound, as discussed by Meyer, has the following thermodynamic values: $\mathrm{pK}_{\mathrm{a} 1}=2$,

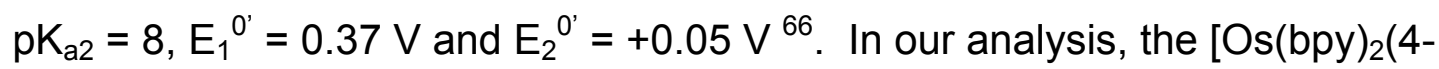
$\mathrm{AMP}) \mathrm{OH}]^{2+}$ redox couple attached to the $\mathrm{SAM}$ exhibits pKa1 $=2.4, \mathrm{pKa} 2=9.3, \mathrm{E}_{1}{ }^{\prime}=+$ $0.3 \mathrm{~V}$ and $\mathrm{E}_{2}^{0^{\prime}}=-0.11 \mathrm{~V}$. The $\mathrm{pH}$ dependent window is extended in our compound by approximately $1 \mathrm{pH}$ unit. This can be attributed to slight changes in the electrostatic interactions of the compound (the loss of resonace in the py-bpy vs tpy ligand). Overall, the thermodynamics behaves well in accordance to the model. As in the galvinol system, there is some scatter in $\mathrm{E}_{r}$ at the higher $\mathrm{pH}$ 's. In this case, only the B$\mathrm{R}$ buffer system was used, and the highest $\mathrm{pH}$ measured was 11 , so it is unlikely that buffer changes or alkaline error are the sources of the scatter. Also, reversible voltammograms at $\mathrm{pH} 11$ remain ideal with respect to peak splitting and peak halfwidths, so thermodynamic heterogeneity is not a likely source. Changes in liquidjunction potential at the reference electrode tip is still a possibility.

Kinetically, the system deviates from the theory in many aspects. The apparent standard rate constant does reach a maximum at $\mathrm{pH}<\mathrm{pK}_{\mathrm{a} 1}$ and $\mathrm{at} \mathrm{pH}>\mathrm{pK}_{\mathrm{a} 2}$, and it does decrease in the region between $\mathrm{pK}_{\mathrm{a} 1}$ and $\mathrm{pK}_{\mathrm{a} 2}$, according to theoretical predictions. However, as can be seen by Figures 4.3.5.13 and 4.3.5.14, the average $\log \mathrm{k}_{\mathrm{s}, \mathrm{app}}$ does not achieve the deep minimum at $\mathrm{pK}_{\text {mid }}$ predicted by the model. It appears that, starting at $\mathrm{pK}_{\mathrm{a} 1}$, the average $\log \mathrm{k}_{\mathrm{s}, \mathrm{app}}$ decreases to a minimum value of 1.2 - 1.5, at $\mathrm{pH} 3-8$. In this $\mathrm{pH}$ region, the average log $\mathrm{k}_{\mathrm{s}, \mathrm{app}}$ remains relatively constant until close to $\mathrm{pK}_{\mathrm{a} 2}$, where it increases up to the value for $\log \mathrm{k}_{\mathrm{s} 2}$. The cause of this 
deviation is unknown. However, it is worth noting that this deviation is similar to the deviation seen in the galvinol system. The possible cause of the deviation will be discussed more thoroughly in the next section.

Despite the clear deviation from the model at intermediate $\mathrm{pH}$, a number of interesting observations can be made about the osmium system. It is interesting to compare the standard rate constant of the osmium system to other systems attached to SAM's. There are 19 atoms (15 $\mathrm{CH}_{2}$ 's) connecting the osmium redox couple to the electrode. Referring to Table 3.5.1, that corresponds to an approximate standard rate constant of 1-6 s $\mathrm{s}^{-1}$ for the ferrocene and Ru complexes. The osmium has a value of 60 $\mathrm{s}^{-1}$ for $\mathrm{k}_{\mathrm{s} 2}$, which is about an order of magnitude higher than expected. The higher standard rate constant for the Osmium complex implies a smaller reorganization energy, compared to reorganization energies of 0.8-1.0 eV for the ferrocene and $\mathrm{Ru}$ complexes. The measured reorganization energies of the Osmium system at the $\mathrm{pH}$ extremes are 0.55-1.0 eV (cathodic) and 0.70-1.0 eV (anodic), as seen in Table 4.3.5.1. Only the cathodic values are less than the value of $0.8 \mathrm{eV}$ calculated for the ferrocene and Ru complexes. Using the smaller value of the cathodic reorganization energy for the osmium system could somewhat account for the higher standard rate constant. According to Marcus DOS theory, a decrease in $\lambda$ of $0.2 \mathrm{eV}$ results in an increase of $k_{s}$ by roughly a factor of 10 . Reorganization energies gathered from the slope of the alternate Tafel analysis (Table 4.3.5.2) yield similar results, with values of 0.65-0.92 $\mathrm{eV}$ at the $\mathrm{pH}$ extremes. Note that this method assumes equal anodic and cathodic reorganization energies. Again, using the smaller values for reorganization 
energy, the difference in $\lambda$ between the Osmium system $(\sim 0.6 \mathrm{eV})$ and the reported $\lambda$ 's for the ferrocene and Ru systems $(\sim 0.8 \mathrm{eV})$ could account for the order of magnitude discrepancy between $k_{s}$ values of the systems $\left(60 \mathrm{~s}^{-1}\right.$ for the osmium system vs around 5-6 for the Ferrocene and Ru systems).

The transfer coefficient at zero overpotential also deviates significantly from the model. At $\mathrm{pH}<\mathrm{pK}_{\mathrm{a} 1}$ and $\mathrm{pH}>\mathrm{pK}_{\mathrm{a} 2}$, where simple electron transfer occurs, $\alpha(0)$ should equal 0.5. As seen in Figure 4.3.5.18, both above $\mathrm{pK}_{\mathrm{a} 2}$ and below $\mathrm{pK}_{\mathrm{a} 1}, \alpha(0)$ is slightly less than $0.5(0.47-0.48)$. However, $\alpha(0)$ does appear to follow the predicted trend in that $\alpha(0)$ at $\mathrm{pK}_{\text {mid }}<\mathrm{pH}<\mathrm{pK}_{\mathrm{a} 2}$ is higher than $\alpha(0)$ at $>\mathrm{pK}_{\mathrm{a} 2}$. Also, at $\mathrm{pK}_{\text {mid }}(\mathrm{pH} 6), \alpha(0)$ is approximately equal to the values above $\mathrm{pK}_{\mathrm{a} 2}$ and below $\mathrm{pK}_{\mathrm{a} 1} . \quad \alpha(0)$ at $\mathrm{pK}_{\mathrm{a} 1}<\mathrm{pH}<$ $\mathrm{pK}_{\text {mid }}$ is slightly less than the value below $\mathrm{pK}_{\mathrm{a} 1}$. Overall the trend appears to be correct, only it appears to be skewed value for $\alpha(0)$ of less than 0.5 , even in the $\mathrm{pH}$ regions wheren only simple electron transfer should occur. Regardless, the deviations of $\alpha(0)$ from 0.5 is predicted to be much larger for reorganization energies of $0.6-0.8 \mathrm{eV}$ than experimentally seen ${ }^{8}$. Because of the considerable scatter among the data points, it was impossible to fit the data to the model. Note that results were similar with the galvinol system. 


\subsection{Discussion of the deviation of $k_{s, a p p}$ for Galvinol and the Osmium Complex}

The analysis of both $1 \mathrm{e} 1 \mathrm{H}$ systems provided mixed results. Thermodynamically, both systems behaved closely to the theoretical model for a $1 \mathrm{e} 1 \mathrm{H}$ system. However, the kinetics of both systems deviated significantly from the theory. Both the galvinol and the Osmium system obeyed theoretical predictions for $\log \mathrm{k}_{\mathrm{s}, \mathrm{app}}$ when close to $\mathrm{pK}_{\mathrm{a} 2}$ (as well as $\mathrm{pK}_{\mathrm{a} 1}$ for the Osmium system). However, as the $\mathrm{pH}$ of each system approached $\mathrm{pK}_{\text {mid }}$, strong deviations from the model became apparent. Specifically, experimental values for the average $\mathrm{k}_{\mathrm{s}, \mathrm{app}}$ decreased at a much slower rate as $\mathrm{pH}$ approached $\mathrm{pK}_{\text {mid }}$ than predicted by theory. With the galvinol system, the apparent standard rate constant decreased as $\mathrm{pH}$ decreased without reaching a minimum (which is likely due to the fact that $\mathrm{pK}_{\text {mid }}$ is unknown, but somewhere outside the $\mathrm{pH}$ range that was accessible). However, with the Osmium system, which allowed access to the entire theoretical $\mathrm{pH}$ range, the apparent standard rate constant reached a minimum with a $\mathrm{k}_{\mathrm{s}, \mathrm{app}}$ at least $1 \frac{1}{2}$ orders of magnitude higher that that predicted by theory (with a potential dependent transfer coefficient, the deviation is even greater). In our initial attempts to account for this discrepancy, a number of hypotheses have been considered. In general iR drop can cause systematic errors, but for the galvinol system, the deviations occurred at pH's where there was no significant iR drop. With the Osmium system, while scan rates were extended to $1000 \mathrm{~V} / \mathrm{s}$ at all pH's, coverages were small enough that iR drop was insignificant even at the highest scan rates. Buffer 
composition and low ionic strength electrolytes were initially considered as sources of the deviation, then rejected when electrolytes of high ionic strength and a single composition were used, and the deviations were still apparent for both systems. Since the $\left[\mathrm{Os}(\mathrm{bpy})_{2}(4-\mathrm{AMP}-\mathrm{SAM}) \mathrm{Cl}\right]^{+/ 2+}$ complex exhibits kinetic behavior $\left(\mathrm{k}_{\mathrm{s}}\right.$ and $\left.\lambda\right)$ independent of $\mathrm{pH}$, it is unlikely that the deviation is attributable to the monolayer or attachment chemistry. At this point, since we have exhausted the likely experimental causes, we must consider the possibility that the source of the problem might lie in the theoretical model itself. There were two main assumptions initially made by Laviron and kept in the Finklea model. The first assumption is that proton transfer is at equilibrium. In other words, proton transfer is much faster than electron transfer, thus any limiting contribution to the rate would be due to the electron transfer contribution. The second main assumption is that electron transfer and proton transfer occur as separate discrete steps. The validity of both of these assumptions are explored.

With respect to the idea that proton transfer is not at equilibrium (and thus contributing to the overall rate), there are a number of reasons that this is not the likely cause of the deviations in our results. The first argument is obtained simply by recognizing what kind of effect slow proton transfer would have on the apparent standard rate constant. There would be two general cases when slow proton transfer would affect the apparent standard rate constant. The first case is when the rate of proton transfer is in fact slower than the rate of electron transfer. In this case, the apparent standard rate constant would be then limited by the slower rate, thus providing an erroneously low result. The deviation in experimental data provides an 
erroneously high result, thus this is unlikely the source of the problem. The second case is where the rate of proton transfer is higher than the rate of electron transfer, but the rate of electron transfer approaches the rate of proton transfer. A theoretical analysis by Dr. Finklea indicates that this assumption results in highly asymmetric Tafel plots, but that the standard rate constant would not be affected ${ }^{31}$.

Discrete proton coupled electron transfer is the commonly accepted mechanism in current research. The evidence in this paper suggests that the assumption that the proton and transfer steps occur discretely might not be valid. Thus, at $\mathrm{pK}_{\mathrm{a} 1}<\mathrm{pH}<$ $\mathrm{pK}_{\mathrm{a} 2}$, the relevant mechanistic step is a concerted transfer of the electron and the proton: $\mathrm{OX}+\mathrm{e}^{-}+\mathrm{H}^{+} \leftrightharpoons$ RED. Recently, there has been considerable theoretical ${ }^{99-101}$ and experimental work ${ }^{102-104}$ on homogenous PCET reactions. The theoretical treatments of Hammes-Schiffer ${ }^{100 ; 101}$ in particular indicate a strong coupling of the proton transfer step to the electron transfer step. This is the first study of a $1 \mathrm{e} 1 \mathrm{H}$ system, in which the results are not biased or distorted by adsorption, double layer effects and mass transfer. It remains to be seen whether these theories can account for the anomalous behavior of the apparent standard rate constant for the galvinol and Osmium systems.

\subsection{Future Directions}

As a project, the study of the $1 \mathrm{e} 1 \mathrm{H}$ system is far from complete. The Osmium redox couple is an ideal $1 \mathrm{e} 1 \mathrm{H}$ system to investigate. We were able to look at all 
important $\mathrm{pH}$ regions (low, moderate, and high). We were able to determine all of the thermodynamic parameters, with great success $\left(\mathrm{pK}_{\mathrm{a} 1}, \mathrm{pK}_{\mathrm{a} 2}, \mathrm{E}_{1}{ }^{0^{\prime}}\right.$, and $\left.\mathrm{E}_{2}{ }^{0^{\prime}}\right)$. We were also able to calculate some of the kinetic parameters $\left(\mathrm{k}_{\mathrm{s} 1}, \mathrm{k}_{\mathrm{s} 2}, \lambda\right.$, and $\left.\alpha(0)\right)$. In the galvinol project, we were able to calculate the thermodynamic and kinetic parameters associated with the intermediate/high $\mathrm{pH}$ interface $\left(\mathrm{pK}_{\mathrm{a} 2}, \mathrm{E}_{2}{ }^{{ }^{\prime}}\right.$, and $\left.\mathrm{k}_{\mathrm{s} 2}\right)$.

Thermodynamic and kinetic parameters at the low/intermediate $\mathrm{pH}$ interface $\left(\mathrm{pK}_{\mathrm{a} 1}, \mathrm{E}_{1}{ }^{{ }^{\prime}}\right.$, $\mathrm{ks} 1, \lambda$, and $\alpha(0))$ were unavailable because they were outside the accessible $\mathrm{pH}$ range.

We were able to gather $\alpha(0)$ data at the intermediate/high $\mathrm{pH}$ interface, but were unable to pin down the value for the reorganization energy, which has importance in comparing galvinol to other redox systems. It would be desirable in a future project to determine this reorganization energy. This could be done using equipment that can collect voltammograms at very high scan rates. With current technology, using microelectrodes, CV's can be routinely collected at greater than 50,000 V/s. However the cost of equipment makes this route prohibitive. The other alternative is to synthesize the galvinol attached to a longer tether. General preparatory procedures for galvinol have been published ${ }^{45 ; 4}$, and through further collaboration with Dr. Shultz, this would be reasonable to achieve.

Significant deviations from the kinetic model for the galvinol and Osmium systems occured at intermediate $\mathrm{pH}$, both in the standard rate constants and the transfer coefficients. The inability to account for this discrepancy puts some of the results in question. In future work, it would be desirable to try to account for the deviations from the model. It might be worthwhile to study the Osmium system, using a 
shorter tether, which would result in much higher value for the standard rate constants. The purpose of this analysis would be to determine whether the anomalously high minimum in the apparent rate constant over the majority of the intermediate $\mathrm{pH}$ range was due to a systematic error associated with the data collection. Deviation in the apparent standard rate constants for both the galvinol and Osmium systems begins to occur at a value of $10-30 \mathrm{~s}^{-1}$. It is possible that the electron transfer mechanism changes from stepwise to concerted at rate constants around this value. Data using an Osmium redox couple with a higher $\mathrm{k}_{\mathrm{s} 2}$ value would quickly resolve this issue. If anomalous kinetic behavior started occurring at the same $\mathrm{pH}$ (which would correspond to a higher $\mathrm{k}_{\mathrm{s}, \mathrm{app}}$ ), the anomaly may not be due to a change in mechanism.

The validity of the assumption of discrete electron/transfer project clearly needs to be evaluated. The study of other model systems is needed to do this. A number of possible systems exist which could be used. The most promising deals with replacing the water from our Osmium complex, $\left[\mathrm{Os}(\mathrm{bpy})_{2}(4-\mathrm{AMP}) \mathrm{OH}_{2}\right]^{2+}$, with a different ionizable ligand. The aqua ligand had some difficulty (because of the second ionizable hydrogen) in that at very high $\mathrm{pH}(>12)$, the iii/iv wave starts to merge with the ii/iii wave, which limits the high $\mathrm{pH}$ analysis. A number of ionizable ligands which could work with varying success have been reported ${ }^{14}$. A promising duo appears to be imidazole and benzimidazole. Both of these ligands have only one ionizable site, so there would be no interfering $\mathrm{Os}^{3+/ 4+}$ waves. Ruthenium analogs using these two ligands are of interest, based on published Pourbaix diagrams ${ }^{14}$, because the imidazole analog would allow the gathering of information at the low/intermediate $\mathrm{pH}$ 
interface, while the benzimidazole analog would allow the collection of information at the intermediate/high $\mathrm{pH}$ interface. It is possible that similar Osmium complexes could be designed as well.

This project is somewhat open-ended in the sense that there are numerous directions that it can follow. After successful analysis of the $1 \mathrm{e} 1 \mathrm{H}$ system, it is a natural progression to study more complex redox couples $(2 \mathrm{e} 1 \mathrm{H}, 1 \mathrm{e} 2 \mathrm{H}, 2 \mathrm{e} 2 \mathrm{H}$, etc). Theoretical predictions by Finklea ${ }^{8 ; 9 ; 30}$ have been made for these more complex systems, and it is desirable to experimentally assess these systems.

\subsection{Conclusions}

Proton coupled electron transfer reactions are prominent in a number of chemical and biological processes. Analyses of these kinds were based upon a stepwise mechanism for electron and proton transfer with a constant transfer coefficient. This work explored the consequences of a potential-dependent transfer coefficient using a stepwise mechanism on redox couples attached to electrodes by self-assembled monolayers. Derivation of the potential-dependent transfer coefficient originated from the Marcus density-of-states model of heterogeneous electron transfer. In this work we explored two systems that exhibited PCET: the organic redox couple

galvinol, and the inorganic redox couple $\left[\mathrm{Os}(\mathrm{bpy})_{2}(4-\mathrm{AMP}) \mathrm{OH}_{2}\right]^{2+/ 3+}$. Both redox couples behaved as $1 \mathrm{e} 1 \mathrm{H}$ systems. The thermodynamics and kinetics of these species were explored, with mixed results. Thermodynamically, both systems behaved according to the model. However, the kinetics of both systems deviated significantly 
from the model. The deviation is that at intermediate $\mathrm{pH}$, the apparent standard rate was significantly higher than predicted by the model. We think that this suggests that there is a fundamental error in the assumption of stepwise electron transfer. More work is required to account for the discrepancies between the model and the theoretical results. 


\section{Reference List}

(1) Reiger, P. A. Electrochemistry, 2nd Edition; Chapman \& Hall: New York, 1994.

(2) Bard, A. J.; Faulkner, L. R. Electrochemical Methods: Fundamentals and Applications; John Wiley \& Sons, Inc.: 1980.

(3) Gileadi, E. Electrode Kinetics for Chemists, Chemical Engineers, and Materials Scientists; VCH Publishers, Inc.: 1993.

(4) Monk, P. Fundamentals of Electroanalytical Chemistry; John Wiley \& Sons: 2001.

(5) Miller, R. J. D.; McLendon, G. L.; Nozik, A. J.; Schmickler, W.; Willig, F. Surface Electron Transfer Processes; VCH Publishers, Inc.: 1995.

(6) Miller, C. J. Heterogenous Electron Transfer Kinetics at Metallic Electrodes; In Physical Electrochemistry: Principles, Methods, and Applications; Rubinstein, I., ed. Marcel Dekker, Inc.: New York, 2002; pp 27-80.

(7) Miller, C. J. Heterogenous Electron Transfer Kinetics at Metallic Electrodes; In Physical Electrochemistry: Principles, Methods, and Applications; Rubinstein, I., ed. Marcel Dekker, Inc.: New York, 1995; pp 27-80.

(8) Finklea, H. O. J.Electroanal.Chem. 2001, 495, 79-86.

(9) Finklea, H. O. Studies of Electron Transfer kinetics at Electrodes Coated with Self-assembled Monolayers; In Volume 10: Modified Electrodes; 2001.

(10) Parker, S. P. McGraw-Hill Dictionary of Chemical Terms; McGraw-Hill Book Co.: 1984.

(11) Finklea, H. O. Electroanal.Chem. 1996, 19, 109-335.

(12) Forster, R. J.; Faulkner, L. R. J.Am.Chem.Soc. 1994, 116, 5444-5452.

(13) Brevnov, D. Development and Application Electrochemical and Spectrophotometric Methods Based upon AC Potential Modulation for Charactarization of Hybrid Bilayer Membranes and Electroactive Self-Assembled Monolayers Supported on Gold Electrodes; 2000.

(14) Goldsby, K. A.; Slattery, S. J.; Blaho, J. K.; Lehnes, J. Coord.Chem.Rev. 1998, 174, 391-416.

(15) Okamura, M. Y.; Graige, M. S.; Paddock, M. L.; Bruce, J. M. J.Am.Chem.Soc. 1996, 118, 9005-9016.

(16) Stowell, M. H. B.; McPhillips, T. M.; Rees, D. C.; Soltis, S. M.; Abresch, E.; Feher, G. Science 1997, 276, 812-815.

(17) Rutherford, A. W.; Faller, P. Trends Biochem.Sci. 2002, 26, 341-344.

(18) anon. Dopamine - A Sample Neurotransmitter. http://www.utexas.edu/research/asrec/dopamine.html . 2002. 
(19) DuVall, S. H.; McCreery, R. L. J.Am.Chem.Soc. 2000, 122, 6759-6764.

(20) Hildebrandt, P.; Murgida, D. H. J.Am.Chem.Soc. 2001, 123, 4062-4068.

(21) Laviron, E. J.Electroanal.Chem. 1979, 101, 19-28.

(22) Laviron, E. J.Electroanal.Chem. 1979, 97, 135-149.

(23) Laviron, E. J.Electroanal.Chem. 1980, 109, 57-67.

(24) Laviron, E. J.Electroanal.Chem. 1980, 115, 65-74.

(25) Laviron, E. J.Electroanal.Chem. 1982, 134, 205-212.

(26) Laviron, E. J.Electroanal.Chem. 1983, 146, 15-36.

(27) Laviron, E. J.Electroanal.Chem. 1983, 157, 7-18.

(28) Laviron, E. J.Electroanal.Chem. 1984, 169, 23-28.

(29) Laviron, E. J.Electroanal.Chem. 1984, 169, 29-46.

(30) Finklea, H. O. J.Phys.Chem.B 2001, 105, 8685-8693.

(31) Haddox, Robert M. and Finklea, H. O. Proton Coupled Electron Transfer (PCET) of Galvinol in Self-Assembled Monolayers. Journal of Electroanalytical Chemistry . 2002.

(32) Finklea, H. O.; Haddox, R. M. Physical Chemistry Chemical Physics 2001, 3, 3431-3436.

(33) Neuman, M. R.; Liu, C.-C.; Bocchicchio, B. C.; Overmyer, P. A. Science 1980, 207, 188-189.

(34) Penner, R. M.; Heben, M. J.; Longin, T. L.; Lewis, N. S. Science 1990, 250, 1118-1122.

(35) Bard, A. J.; Mirkin, M. V.; Unwin, P. R.; Wipf, D. O. J.Phys.Chem. 1992, 96, 1861-1868.

(36) Feldberg, S. W.; Rubinstein, I. J.Electroanal.Chem. 1988, 240, 1-15.

(37) Smith, C. P.; White, H. S. Anal.Chem. 1992, 64, 2398-2405.

(38) Shultz, D. A.; Sagara, T.; Midorikawa, T.; Zhao, Q. Langmuir 1998, 14, 3682-3690.

(39) Forster, R. J.; Faulkner, L. R. Anal.Chem. 1995, 67, 1232-1239.

(40) Abruna, H. D.; Bretz, R. L.; Acevedo, D.; Tirado, J. D. Langmuir 1994, 10, 1971-1979.

(41) Forster, R. J. J.Electrochem.Soc. 1997, 144, 1165-1173.

(42) Nishimura, N.; Iga, S.; Satoh, M.; Yamamoto, S. Bull.Chem.Soc.Jpn. 1992, 65, 2437-2441.

(43) Richards, j. A.; Whitson, P. E.; Evans, D. H. J.Electroanal.Chem. 1975, 63, 311-327. 
(44) Kharasch, M. S.; Joshi, B. S. Reactions of Hindered Phenols. I. Reactions of 4,4'-Dihydroxy3,5,3',5',-tetra-tert-butyl Diphenylmethane; In Hindered Phenols 1.; 1957; pp 1435-1438.

(45) Shultz, D. A.; Tew, G. N. J.Org.Chem. 1994, 59, 6159-6160.

(46) Shultz, D. A.; Zhao, Q. Tetrahedron Lett. 1996, 37, 8837-8840.

(47) Tamura, M.; Kinoshita, M.; Hosokoshi, Y. Pressure effect on organic radicals; In Molecular Crystals and Liquid Crystals Science and Technology, Section A: Molecular Crystals and Liquid Crystals; 1997; pp 423-430.

(48) Tyutyulkov, N.; Dietz, F.; Madjarova, G.; Muellen, K. J.Phys.Chem.B 2000, 104, 7320-7325.

(49) Perekhodtsev, Gleb D. Organic and molecular ferromagnetism (a review). http://center.chph.ras.ru/ perekhod/review6e.html . 2002.

(50) Smith, M. A. I.; Marley, K. A.; Seigler, D.; Singletary, K. W.; Meline, B. J.Food Sci. 2000, 65, 352-356.

(51) Schwarz, K.; Bertelsen, G.; Gardner, P. T.; Huynh-Ba, T.; Tijburg, L.; Heionen, M. I. Eur.Food.Res. Technol 2001, 212, 319-328.

(52) Preparative Organic Chemistry; Hilgeteg and Martin: 1972.

(53) Rived, F.; Roses, M.; Bosch, E. Anal.Chim.Acta 1998, 374, 309.

(54) Chambers, J. Q. The Chemistry of Quinonoid Compounds; John Wiley: New York, 2002.

(55) Becka, A. M.; Miller, C. J. J.Phys.Chem. 1993, 97, 6233-6239.

(56) Becka, A. M.; Miller, C. J. J.Phys.Chem. 1992, 96, 2657-2668.

(57) Terrettaz, S.; Becka, A. M.; Traub, M. J.; Fettinger, J. C.; Miller, C. J. J.Phys.Chem. 1995, 99, 11216-11224.

(58) Yang, D.; Zi, M.; Chen, B.; Gao, Z. J.Electroanal.Chem. 1999, 470, 114-119.

(59) Armstrong, F. A.; Allen, J. W. A.; Jameson, G. N. L.; Hirst, J. J.Am.Chem.Soc. 1998, 120, 11994-11999.

(60) Armstrong, F. A.; Burgess, B. K.; Kemper, M. A.; Jameson, G. N. L.; Duff, J. L. C.; Hirst, J. J.Am.Chem.Soc. 1998, 120, 7085-7094.

(61) Bell-Loncella, E. T.; Bessel, C. A. Inorg.Chim.Acta 2000, 303, 199-205.

(62) Angus, J. C.; Lu, B.; Zappia, M. J. J.Appl.Electrochem. 1987, 17.

(63) Mohanty, J. G.; Chakravorty, A. Inorg.Chem. 1976, 15, 2912-2916.

(64) Mohanty, J. G.; Chakravorty, A. Inorg.Chem. 1977, 16, 1561-1563. 
(65) Meyer, T. J.; Geselowitz, D. A.; Pipes, D. W.; Takeuchi, K. J.; Dobson, J. C. Inorg.Chem. $1986,25,2357-2365$.

(66) Meyer, T. J.; Pipes, D. W.; Thompson, M. S.; Takeuchi, K. J. Inorg.Chem. 1984, 23, 18451851.

(67) Abruna, H. D.; Acevedo, D.; Bretz, R. L.; Tirado, J. D. Langmuir 1994, 10, 1300-1305.

(68) Abruna, H. D.; Bretz, R. L. J.Electrochem.Soc. 1995, 388, 123-132.

(69) Abruna, H. D.; Acevedo, D. J.Phys.Chem. 1991, 95, 9590-9594.

(70) Finklea, H. O.; Hanshew, D. D. J.Am.Chem.Soc. 1993, 114, 3173-3181.

(71) Finklea, H. O.; Ravenscroft, M. S.; Snider, D. A. Langmuir 1993, 9, 223-227.

(72) Finklea, H. O.; Ravenscroft, M. S. Israel J.Chem. 1997, 37, 179-184.

(73) Finklea, H. O.; Liu, L.; Ravenscroft, M. S.; Punturi, S. J.Phys.Chem. 1996.

(74) Finklea, H. O.; Hanshew, D. D. J.Electroanal.Chem. 1993, 347, 327-340.

(75) Forster, R. J. Inorg.Chem. 1996, 35, 3394-3403.

(76) Calvert, J. M.; Schmehl, R. H.; Sullivan, B. P.; Facci, J. S.; Meyer, T. J.; Murray, R. W. Inorg.Chem. 1983, 22, 2151-2162.

(77) Forster, R. J.; Keyes, T. E.; Vos, J. G. Analyst 1998, 123, 1905-1911.

(78) Meyer, T. J.; Sullivan, B. P.; Caspar, J. V.; Kober, E. M. Inorg.Chem. 1998, 27, 4587-4598.

(79) Constable, E. C.; Cargill Thompson, A. M. W. J.Chem.Soc., Dalton Trans. 1995, 1615-1627.

(80) Demadis, K. D.; El-Samanody, E. S.; Meyer, T. J.; White, P. S. Polyhedron 1999, 18, $1587-$ 1594.

(81) Theilacker, Bill. The Synthesis and Charactarization of $[\mathrm{Os}(\mathrm{tpy})(\mathrm{bpy})(\mathrm{L})] \mathrm{L}=(\mathrm{H} 20$ or $\mathrm{OH}-)$. 1998.

(82) Broomhead, J. A.; Dwyer, F. P. Aust.J.Chem. 1961, 14, 250-252.

(83) Buckingham, D. A.; Dwyer, F. P.; Sargeson, A. M. Aust.J.Chem. 1964, 17, 622-631.

(84) Sargeson, A. M.; Dixon, N. E.; Lawrance, F. A. Trifluoromethanesulfonates and trifluoromethanesulfonato-o complexes; In Inorganic Synthesis, Vol 24; Shreeve, J. M., ed. 1986; pp 243-249.

(85) Brewer, K. J.; Vogler, L. M. Inorg.Chem. 1996, 35, 818-824.

(86) Forster, R. J.; Faulkner, L. R. J.Am.Chem.Soc. 1994, 116, 5453-5461.

(87) Forster, R. J.; Faulkner, L. R. Langmuir 1995, 11, 1014-1023. 
(88) Forster, R. J.; O'Kelly, J. P. J.Phys.Chem. 1996, 100, 3695-3704.

(89) Abruna, H. D.; Arana, C. R. Inorg.Chem. 1993, 32, 194-203.

(90) Abruna, H. D.; Maskus, M. Langmuir 1996, 12, 4455-4462.

(91) Anson, F. C.; Campbell, J. L. E. Langmuir 1996, 12, 4008-4014.

(92) Forster, R. J.; Loughman, P.; Keyes, T. E. J.Am.Chem.Soc. 2000.

(93) Buckingham, D. A.; Dwyer, F. P.; Goodwin, H. A.; Sargeson, A. M. Aust.J.Chem. 1964, 17, 325-336.

(94) anon. Carabodiimide coupling using EDC. http://chem.ch.huji.ac.il/ eugeniik/edc.htm . 2002.

(95) Calmes, M.; Glot, C.; Michel, T.; Rolland, M.; Martinez, J. Tetrahedron 2000, 11, 737-741.

(96) Garcia, H.; Miranda, M. A.; Alvaro, M.; Primo, J. Tetrahedron 1992, 48, 3437-3444.

(97) Forster, R. J.; Figgemeier, E.; Lees, A. C.; Hjelm, J.; Vos, J. G. Langmuir 2000, 16, 78677870.

(98) Forster, R. J.; Figgemeier, E.; Loughman, P.; Lees, A. C.; Hjelm, J.; Vos, J. G. Langmuir 2000, $16,7871-7875$.

(99) Cukier, R. I.; Nocera, D. G. Ann.Rev.Phys.Chem 1998, 49, 337-369.

(100) Hammes-Schiffer, S. Acc.Chem.Res. 2001, 34, 273-281.

(101) Iordanova, N.; Decornez, S.; Hammes-Schiffer, S. J.Am.Chem.Soc. 2001, 123, 3723-3733.

(102) Roth, J. P.; Lovell, S.; Mayer, J. M. J.Am.Chem.Soc. 2000, 122, 5486-5498.

(103) Lebeau, E. L.; Binstead, R. A.; Meyer, T. J. J.Am.Chem.Soc. 2001, 123, 10535-10544.

(104) Binstead, R. A.; McGuire, M. E.; Dovletoglou, A.; Seok, W. K.; Roecker, L. E.; Meyer, T. J. J.Am.Chem.Soc. 1992, 114, 173-186. 